\title{
Regional versus global integration of euro-zone retail banking markets: understanding the recent evidence from price-based integration measures
}

Citation for published version (APA):

Kleimeier, S., \& Sander, H. (2005). Regional versus global integration of euro-zone retail banking markets: understanding the recent evidence from price-based integration measures. METEOR, Maastricht University School of Business and Economics. METEOR Research Memorandum No. 032 https://doi.org/10.26481/umamet.2005032

Document status and date:

Published: 01/01/2005

DOI:

10.26481/umamet.2005032

Document Version:

Publisher's PDF, also known as Version of record

Please check the document version of this publication:

- A submitted manuscript is the version of the article upon submission and before peer-review. There can be important differences between the submitted version and the official published version of record.

People interested in the research are advised to contact the author for the final version of the publication, or visit the DOI to the publisher's website.

- The final author version and the galley proof are versions of the publication after peer review.

- The final published version features the final layout of the paper including the volume, issue and page numbers.

Link to publication

\footnotetext{
General rights rights.

- You may freely distribute the URL identifying the publication in the public portal. please follow below link for the End User Agreement:

www.umlib.nl/taverne-license

Take down policy

If you believe that this document breaches copyright please contact us at:

repository@maastrichtuniversity.nl

providing details and we will investigate your claim.
}

Copyright and moral rights for the publications made accessible in the public portal are retained by the authors and/or other copyright owners and it is a condition of accessing publications that users recognise and abide by the legal requirements associated with these

- Users may download and print one copy of any publication from the public portal for the purpose of private study or research.

- You may not further distribute the material or use it for any profit-making activity or commercial gain

If the publication is distributed under the terms of Article $25 \mathrm{fa}$ of the Dutch Copyright Act, indicated by the "Taverne" license above, 


\title{
Regional Versus Global Integration of Euro-Zone Retail Banking Markets:
}

\section{Understanding the Recent Evidence from Price-Based Integration Measures ${ }^{1}$}

\author{
by
}

\author{
Stefanie Kleimeier, ${ }^{\mathrm{a}, \mathrm{b}}{ }^{*}$ and Harald Sander ${ }^{\mathrm{b}, \mathrm{c}}$
}

\begin{abstract}
${ }^{a}$ Limburg Institute of Financial Economics, Maastricht University, Tongersestraat 53, 6211 LM Maastricht, The Netherlands.

${ }^{\mathrm{b}}$ METEOR, Maastricht University, Tongersestraat 53, 6211 LM Maastricht, The Netherlands.

${ }^{\mathrm{c}}$ Faculty of Economics and Business Administration, University of Applied Sciences Cologne, Claudiusstr.1, 50678 Köln, Germany.
\end{abstract}

\begin{abstract}
This study investigates the current state of euro-zone banking market integration by comparatively applying convergence and cointegration measures to mortgage and corporate loan markets. As an innovation to the literature, convergence measures are exposed to a difference-in-differences methodology which allows separating euro zone-specific from global integration effects. We find that euro-zone convergence of retail banking interest rates has so far largely been a result of integrating wholesale markets after the elimination of exchange rate risks. Moreover, some convergence is found to be part of a global process rather than euro zonespecific. After 1999 some evidence for stronger integration is detected for a restricted "convergence club" excluding Germany, Ireland and Belgium. We conclude in favor of using margin-convergence measures and cointegration measure for assessing the regional integration progress in relation to global banking market integration.
\end{abstract}

JEL Classification Numbers: E42; E43; E52; E58; F15; F36.

Keywords: European Financial Integration, Global Financial Integration, Retail Banking, Convergence, Cointegration, Interest Rate Margins, Difference-in-Differences Analysis.

* Corresponding author. Tel: +31-43-3883733; fax: +31-43-3884875

E-mail address: s.kleimeier@berfin.unimaas.nl (Stefanie Kleimeier)

\footnotetext{
${ }^{1}$ We are grateful for the comments made by the discussant of this paper and the participants of the $3^{\text {rd }}$ INFINTI Conference "Real and Financial Aspects of Financial Integration" held in Dublin, June 24, 2005. All remaining errors are ours.
} 


\section{$\underline{1 . \text { Introduction }}$}

The European single market project has promised to create a regionally integrated retail banking market. After it became increasingly evident that a unified European banking market was emerging only slowly - if at all - (Centeno and Mello 1999, Kleimeier and Sander 2000, Economic and Financial Council 2002, Schüler and Heinemann 2002), the unifying potential of a single currency and a single central bank has often been highlighted (see e.g. Padoa-Schioppa 2000). Although more recent evidence reveals first signs of a more integrated euro-zone retail banking market after the introduction of the Euro, these developments are often restricted to some segments of retail banking such as lending to corporations (Kleimeier and Sander 2003, Baele et al. 2004). Meanwhile, global integration of financial markets has also increased considerably. For example, cross-border lending as well as cross-border mergers and acquisitions between the euro zone and the rest of the world have at times been more flourishing than within the euro zone. ${ }^{2}$ Thus, a first and largely unexplored issue that we will address in this paper is to what extent the observed increases in integration are regional or global phenomena.

According to a recent survey article (Baele et al. 2004) evidence on banking market integration can be obtained from quantity-based, price-based and news-based measures of integration. ${ }^{3}$ Quantity-based measures focus on institutional characteristics such as crossborder banking activities, the number and market shares of foreign banks, or bank mergers. Such studies typically conclude that the integration process is still very incomplete (see e.g. Cabral et al. 2002). Particularly, there is still only very limited evidence of cross-border loans and deposits to non-banks within the euro zone but recent studies found a slightly increasing

\footnotetext{
${ }^{2}$ For example, from 1995 to 1999 85\% of all euro zone bank mergers were domestic, reflecting national consolidation mainly among small banks. Among the remaining $15 \%$ cross-border mergers, those involving a non-euro zone bank dominated with $10 \%$. This latter share has increased even further to $17 \%$ in the first half of 2000 (ECB 2000). For cross-border retail activities in the loan and deposit markets Manna (2004) reveals the prominent role of the UK: UK-euro zone cross-border activities relative to all cross-border activities amount to $25 \%$ to $30 \%$ in both markets.
} 
trend towards more cross-border lending which seems, however, to be driven by non-euro zone banks. ${ }^{4}$ Price-based measures of integration often use the law of one price as a point of reference and are, consequently, focusing on the evolution and potential convergence of retail banking interest rate levels. These studies typically find some evidence for integration, which is, however, difficult to reconcile with the obtained cross-border lending picture, which signals a lack of integration. Alternative price-based measures are either focusing on bank margins or are using cointegration methodology. Margins offer a better understanding of the (impact of the) integration process than retail rate levels in periods when market interest rate are converging due to a reduction and final elimination of the exchange rate risks in a monetary union. Only under the condition of a monetary union, both approaches are equivalent. Although the current research (Cabral et al. 2002, Freixas 2003, and Baele et al. 2004) shows some reductions in margins, the significant differences remaining across countries point to the fragmented nature of retail markets. Cointegration studies do not require full convergence of banking product prices, i.e. they explicitly allow for heterogeneity in banking products across countries and for a lack of homogeneity in the data base (Centeno and Mello 1999, Kleimeier and Sander 2000 and 2003, Schüler and Heinemann 2002). These studies typically find less signs for an integrated euro-zone banking market than studies using convergence measures. However, some of these studies also find first signs for more integration in the EMU-period, particularly with reference to lending to the corporate sector. Kleimeier and Sander (2003) hypothesize that these co-movements of interest rates are predominantly a potential consequence of the already highly integrated euro-zone money (and bonds) market under the condition that these retail interest rates react uniformly to changes in

\footnotetext{
${ }^{3}$ For a detailed overview of different integration measures see also Adam et al. (2002) Table 4.1 or Schüler and Heinemann (2002) Table 1.

${ }^{4}$ For example, the share of cross border loans and deposits to/from the euro zone non-financial sector amounted to $2.3 \%$ and $6.0 \%$ of all loans in 1998 but has hardly changed to $3.2 \%$ and $5.2 \%$ in 2002 , respectively. In contrast, interbank and security market cross-border activities have been steadily increasing over time and reached shares between 10\% and 35\% in 2002 (Manna 2004). Furthermore Baele et al. (2004) report that the
} 
market rates. Thus, the second issue we will address in this paper is to give a comparative evaluation of convergence and cointegration measures of integration.

According to Baele et al. (2004: 61) "the proportion of interest rate changes that is explained by common factors provides an alternative measure of the degree of integration". The common factor chosen in these news-based-measure studies is typically a market interest rate either in a benchmark country or in the whole euro zone. Some studies select as the common factor market interest rates that are most closely related to the retail interest rate under investigation (De Bondt 2002, De Bondt et al. 2003, and Baele et al. 2004). Other studies opt for a money market rate as the common (monetary policy) factor (Mojon 2000, Heinemann and Schüler 2003, Sander and Kleimeier 2002, 2004, Toolsema et al. 2002). Despite considerable differences in the methodological approach ${ }^{5}$, such pass-through studies typically find very limited evidence for integration in lending to households and mortgage lending. The situation is slightly better for corporate loans and time deposits. Most importantly, there is some evidence for increasing integration in the latter markets. ${ }^{6}$ Thus, the third issue we will address in this paper is to provide an evaluation of the role of wholesale market integration for retail banking market integration.

Our study will address these three issues by investigating and comparing global and regional integration of mortgage and short-term corporate loan markets by means of three price-based integration measures: those measuring price convergence, those measuring convergence of bank margins and those using cointegration methodology. We collect mortgage and corporate loan rates for each of the euro-zone countries including Austria,

share of cross-border loans from non-euro zone banks (approximately 8\% of all domestic loans in 2002) is higher than the share of cross-border loans from euro zone banks (approximately 4\%).

5 For a comparison of both approaches and a suggestion for a unifying approach see Sander and Kleimeier (2004).

${ }^{6}$ Note that the results that Baele et al. (2004) obtain from their news-based approach also stand in contrast to their price-convergence results. Their analysis indicates segmentation for consumer and mortgage rates and more integration for short and long corporate loan rates and time deposits. Their convergence analysis, on the other hand, concludes differently. Here the cross-sectional standard deviation at the end of the sample period is largest 
Belgium, Finland, France, Germany, Ireland, Italy, Netherlands, Portugal, and Spain and for selected non-euro zone countries including Australia, Canada, Japan, Switzerland, United Kingdom, and the USA. For all these countries monthly interest rates are available for a sample period of January 1995 to December 2002. Margins are calculated over the 10-year bond yield in case of mortgages and 3-months money market rate in case of short-term corporate loans. See Table A1 of the appendix for details. ${ }^{7}$ Instead of a simple before-after analysis of price and margin convergence measures we apply a difference-in-differences approach. This approach - presented here for the first time for retail banking markets - avoids the mistake of over-identifying euro-zone convergence and provides results for euro-zone convergence that can truly be attributed to the European integration efforts rather than being part of a global integration process (chapter 2). Secondly, we conduct various and extensive cointegration analyses for which we also compare the global with the regional euro-zone evolution (chapter 3). This allows us, third, to explicitly compare both, convergence and cointegration measures of integration (chapter 4). Our analysis will show that despite some euro zone-specific convergence the lack of integration in retail markets is still severe. Further wholesale market integration together with a more homogeneous pass-through process appear to be essential conditions for eliminating unexploited cross-border arbitrage. We thus discuss these issues in more detail in chapter 5. Chapter 6 concludes.

\section{Price convergence measures of integration}

\subsection{Advantages and disadvantages of price convergence measures}

Price-based measures have been advocated for a variety of reason, but particularly because these data are readily and more easily available and are typically more accurate than

for short corporate loans and consumer loans (2.0), somewhat lower for long corporate loans (1.5), and low for mortgages and time deposits $(0.5)$. 
quantity-based measures. They also allow for a more straightforward interpretation and may be better able to reveal long-term trends. Adam et al. (2002) therefore suggest using retail rate convergence measures to monitor the progress in European retail banking market integration. The European Commission (2004) has followed this suggestion in its annual Financial Integration Monitor report. For a first impression, consider the development of nominal retail interest rates in the euro zone as presented in Panel A and $\mathrm{C}$ of Figure 1. There appears to be some convergence which has mostly taken place in the mid-1990s. Whereas country differences are smallest at the end of 2002 for mortgage rates, short-term corporate loan rates still show differences of up to $6.2 \%$. Within a sub-group of euro-zone countries excluding Germany, Ireland, and Belgium, however, these rates seem to be more aligned. Nevertheless, after more than 10 years of regulatory integration efforts starting with the $2^{\text {nd }}$ banking directive (BD) in 1993 and five years after the introduction of the single currency, full convergence of interest rates has not yet been reached in retail banking markets.

\section{[insert Figure 1 about here]}

For a more formal statistical test, two measures of convergence have been suggested: $\sigma$ and $\beta$-convergence. $\sigma$-convergence measures whether or not interest rates have become more similar over time when compared to each other or to a benchmark rate. In contrast, $\beta$ convergence measures the speed with which national interest rates converge. This latter measure has been borrowed from the economic growth literature. In the context of economic growth, countries with low initial levels of economic development should show higher economic growth than countries with high initial levels of economic development. With

\footnotetext{
${ }^{7}$ Due to the often short- to medium-term nature of the respective national mortgage rates, we also calculate margins over the 3-months money market rate. Unless otherwise indicated, the results for these margins do not differ substantially from those for mortgage margin over bond yields.
} 
respect to banking market integration this implies that countries with initially relatively high interest rates should show faster downward adjustment than countries with already low interest rates. Consequently, both measures are complementary and can provide differing insights. As Adam et al. (2002) note, $\beta$-convergence can be consistent with $\sigma$-divergence. Full convergence should however not even be expected for two major reasons: First, the current database (prior to the changes introduced recently in the MFI statistics ${ }^{8}$ ) is so heterogeneous that cross-country comparisons can easily be misleading. Second, even if we had a good database, differences in banking products, borrower default risks etc. would still imply that loan portfolios would not necessarily be comparable across countries and thus price differentials should be expected. Thus, given the non-applicability of the law of one price in the strict sense in the retail banking market it can be argued that $\sigma$-convergence is a more realistic integration test (Freixas 2003).

Up to date, convergence measures have mostly been applied to euro-zone countries only, though some studies including Adam et al. (2002) report results for a sample including non-euro zone countries. However, a part or all of the convergence of euro-zone interest rates shown in Figure 1 could be caused by globally falling interest rate levels. In particular, mortgage rates have not only converged across the euro zone but also on a global level. As such, a clear differentiation between euro zone-specific and globally occurring convergence is needed. Moreover, a short reflection shows that price convergence can only be applied under the condition of a single currency where exchange rate risks are eliminated. Consider a difference (D) between two retail bank rates (BR) of two countries A and B. This difference can be decomposed into a difference (D1) of market rates MR (such as short-term money market rates) and differences in spread of the bank rate over that market rate (D2):

$$
\mathrm{D}=\mathrm{MR}_{\mathrm{A}}-\mathrm{MR}_{\mathrm{B}}+\left[\left(\mathrm{BR}_{\mathrm{A}}-\mathrm{MR}_{\mathrm{A}}\right)-\left(\mathrm{BR}_{\mathrm{B}}-\mathrm{MR}_{\mathrm{B}}\right)\right]=\mathrm{D}_{1}+\mathrm{D}_{2}
$$

\footnotetext{
${ }^{8}$ For details on these interest rates that are collected in a comparable fashion by all central banks of the euro
} 
Within the euro zone after 1.1.1999 exchange rate risks as measured by $\mathrm{D}_{1}$ equal (almost) zero. For the post-1999 euro zone it is thus sufficient to explore the integration progress by focusing on retail rates. But since these convergence measures fail to take into account exchange rate risk, they can neither benchmark against the pre-euro period nor against developments in countries outside the euro zone. To do so, integration measures based on the differences in bank margins $\mathrm{D}_{2}$ are more informative as they are corrected for exchange rate expectations. Figure 1 reveals that mortgage rate margins have fallen in the euro zone and are by the end of 2002 more in line with global levels. In contrast, short-term corporate loan margins appear to be more stable over time and with the exceptions of Germany, Ireland, and Belgium, euro-zone margins are by late 2002 in line or even below global levels.

\subsection{A difference-in-differences approach to price and bank-margin convergence}

A difference-in-differences approach (DD) analysis can benchmark against both the pre-euro period and non-euro zone countries. Thus it avoids the mistake of over-identifying euro-zone convergence and provides results for euro-zone $\beta$ - and $\sigma$-convergence that can truly be attributed to the European integration efforts. The main reason for the lack of such DD studies can be found in the lack of comparable non-euro zone data. However, as mortgage rates and short-term corporate loan rates are readily available and probably the most comparable retail lending rates available, these two rates provide valuable material for a policy evaluation study.

For measuring convergence we apply both $\beta$ - and $\sigma$-convergence measures in models of differing complexity: First, for the whole sample period; second, allowing for differences before and during EMU; and third, in form of a DD analysis allowing for time- and regionspecific patterns. For reason of comparability with existing studies we conduct the DD 
approach for retail interest rate levels first before conducting the more realistic and more revealing DD analysis for bank lending margins. From the above discussion it is, however, obvious that focusing on retail rate levels is only appropriate for the euro-zone markets under the single currency. For all three models, $\sigma$-convergence employs the monthly standard deviation across interest rates (SD) as an integration proxy. For $\beta$-convergence, recall that countries with large deviations (in absolute terms) are expected to converge faster than countries with relatively small deviations. As such, $\beta$-convergence commonly focuses on the positive or negative deviation of each national interest rate from a mean or benchmark rate.

To analyze convergence in its simplest form for the full sample period, the following two regressions for $\sigma$ - and $\beta$-convergence can be estimated ${ }^{9}$ :

$\mathrm{SD}_{\mathrm{t}}=\mathrm{a}_{1}+\mathrm{b}_{1} \mathrm{~T}+\varepsilon_{\mathrm{t}}$

$\Delta \mathrm{z}_{\mathrm{c}, \mathrm{r}, \mathrm{t}+12}=\mathrm{d}_{1} \mathrm{z}_{\mathrm{c}, \mathrm{r}, \mathrm{t}}+\varepsilon_{\mathrm{c}, \mathrm{r}, \mathrm{t}}$

In equation (1) $\mathrm{T}$ indicates a trend. In equation (2) $\mathrm{z}_{\mathrm{c}, \mathrm{r}, \mathrm{t}}$ is defined as the difference in month $\mathrm{t}$ between the retail interest rate of country $\mathrm{c}$ and the average retail interest rate or margin in region $\mathrm{r}$ to which country $\mathrm{c}$ belongs. The change in this deviation is measured by $\Delta \mathrm{z}_{\mathrm{c}, \mathrm{r}, \mathrm{t}+12}=$ $\mathrm{z}_{\mathrm{c}, \mathrm{r}, \mathrm{t}+12}-\mathrm{z}_{\mathrm{c}, \mathrm{r}, \mathrm{t} \cdot} 10$

In order to investigate whether euro-zone convergence continued or even increased during the EMU period, dummies can be included in the $\sigma$ - and $\beta$-convergence regressions respectively such that

$$
\begin{aligned}
& \mathrm{SD}_{\mathrm{t}}=\mathrm{a}_{1}+\mathrm{a}_{2} \mathrm{D}_{\mathrm{EMU}}+\mathrm{b}_{1} \mathrm{~T}+\mathrm{b}_{2} \mathrm{~T} \mathrm{D}_{\mathrm{EMU}}+\varepsilon_{\mathrm{t}} \\
& \Delta \mathrm{z}_{\mathrm{c}, \mathrm{r}, \mathrm{t}+12}=\mathrm{d}_{1} \mathrm{z}_{\mathrm{c}, \mathrm{r}, \mathrm{t}}+\mathrm{d}_{2} \mathrm{z}_{\mathrm{c}, \mathrm{r}, \mathrm{t}} \mathrm{D}_{\mathrm{EMU}}+\varepsilon_{\mathrm{c}, \mathrm{r}, \mathrm{t}}
\end{aligned}
$$

\footnotetext{
${ }^{9}$ Both the $\sigma$ - and $\beta$-convergence analysis is based on a methodology suggested by Slaughter (2001). He applies this methodology to per capita income convergence before and after trade liberalization.

${ }^{10} \Delta \mathrm{z}_{\mathrm{c}, \mathrm{r}, \mathrm{t}+12}$ indicates the change over the coming 12 months. Note that we employ rolling samples of overlapping 12-months changes.
} 
where $D_{\text {EMU }}$ is the dummy equal to 1 for observations $t$ during the EMU period. ${ }^{11}$ A negative $\mathrm{b}_{2}$ or $\mathrm{d}_{2}$ coefficient indicates additional convergence during the EMU period.

To order to benchmark the convergence process in the euro zone against global trends, a DD analysis is employed based on the following panel regressions:

$$
\begin{aligned}
& \mathrm{SD}_{\mathrm{r}, \mathrm{t}}=\mathrm{a}_{1}+\mathrm{a}_{2} \mathrm{D}_{\mathrm{EMU}}+\mathrm{a}_{3} \mathrm{D}_{\mathrm{r}}+\mathrm{a}_{4} \mathrm{D}_{\mathrm{r}, \mathrm{EMU}}+\mathrm{b}_{1} \mathrm{~T}+\mathrm{b}_{2} \mathrm{~T} \mathrm{D}_{\mathrm{EMU}}+\mathrm{b}_{3} \mathrm{~T} \mathrm{D}_{\mathrm{r}}+\mathrm{b}_{4} \mathrm{~T} \mathrm{D}_{\mathrm{r}, \mathrm{EMU}} \\
& \quad+\varepsilon_{\mathrm{r}, \mathrm{EMU}, \mathrm{t}} \\
& \Delta \mathrm{z}_{\mathrm{c}, \mathrm{r}, \mathrm{t}+12}=\mathrm{d}_{1} \mathrm{z}_{\mathrm{c}, \mathrm{r}, \mathrm{t}}+\mathrm{d}_{2} \mathrm{z}_{\mathrm{c}, \mathrm{r}, \mathrm{t}} \mathrm{D}_{\mathrm{EMU}}+\mathrm{d}_{3} \mathrm{z}_{\mathrm{c}, \mathrm{r}, \mathrm{t}} \mathrm{D}_{\mathrm{r}}+\mathrm{d}_{4} \mathrm{z}_{\mathrm{c}, \mathrm{r}, \mathrm{t}} \mathrm{D}_{\mathrm{r}, \mathrm{EMU}}+\varepsilon_{\mathrm{c}, \mathrm{r}, \mathrm{t}}
\end{aligned}
$$

Two additional dummies are included: $D_{r}$ is a cross-sectional dummy equal to 1 if the dependent variable is measured for region $r=$ euro zone. $D_{r, E M U}$ is a compound dummy equal to 1 for region $\mathrm{r}$ = euro zone and for observations belonging to the EMU period. As such SD and $\Delta \mathrm{z}$ are calculated for euro zone and non-euro zone countries separately. $\sigma$ - and $\beta$ convergence can now be differentiated by region and period based on the estimated coefficients. The results of the DD regressions (5) and (6) are shown in Table 1 for $\sigma$ - and $\beta$ convergence of rates as well as margins. ${ }^{12}$ The actual convergence coefficients are given by region and time together with tests for differences.

\section{[insert Table 1 about here]}

As could already be expected from the inspection of Figure 1, convergence is found for interest rate levels. For example, for euro-zone mortgage rates the simple analysis of regression (1) with an estimated coefficient of $b_{1}=-0.026$ and $d_{1}=-0.270$ would lead to this conclusion as both coefficients are negative and significant. The DD analysis, however,

\footnotetext{
${ }^{11}$ In order to clearly separate the pre-EMU from the EMU period and in order to create two sample periods of equal length, the pre-EMU period is defined as $z_{c, r, t}$ for $t=1 / 95$ to $12 / 97$ and the EMU period as $t=1 / 99$ to 12/01. Note that for any $\mathrm{t}$ in 1998 the range over which $\Delta \mathrm{z}_{\mathrm{c}, \mathrm{r}, \mathrm{t}+1}$ is calculated falls partly into 1999. As such, this period is excluded.

${ }^{12}$ Details on the simpler models of regressions (1) to (4) are presented in Tables A2 and A3 of the appendix.
} 
provides a more differentiated and exact picture. For mortgage rates, $\sigma$-convergence occurs but is limited to the pre-EMU period. In other words, no reduction in standard deviation was achieved under the single currency. On the other hand, $\beta$-convergence occurs in both periods and is even stronger under the single currency. For short-term corporate loan rates, both $\sigma$ and $\beta$-convergence are limited to the pre-EMU period and divergence occurs under the single currency. So far, our euro-zone results are broadly consistent with findings by Adam et al., who find evidence in favor of $\sigma$-convergence for corporate lending rates and $\sigma$ - and $\beta$ convergence for mortgages. However, how much of this convergence or divergence is euro zone-specific? A look at the non-euro zone results reveals that $\sigma$ - and $\beta$-convergence can be found outside the euro zone in both periods and for both rates. As such, the results reported in the literature so far, overestimate euro-zone convergence as global processes are not properly taken into account. In particular, the absence of mortgage-rate $\sigma$-convergence and the $\sigma$ divergence of corporate loan rates under the single currency has to be contrasted with converging global interest rates. Overall we conclude that $\sigma$ - and $\beta$-convergence can be observed for the euro zone but it appears also to be - at least in part - a global phenomenon.

The finding that much of the euro zone-specific $\sigma$-convergence takes place in the preEMU period lead us to conclude that progressively disappearing exchange rate risks prior to the introduction of the single currency are an important driving factor. Thus, only by looking on lending margins we can obtain evidence whether convergence in the pre-EMU period can somehow be related to the impact of an integration process. Turning directly to the DD analysis and contrasting the euro zone with the non-euro zone mortgage margins reveals that $\sigma$-convergence is much stronger globally in the pre-EMU period. As such no euro zonespecific $\sigma$-convergence can be found. This stands in contrast to the mortgage-rate level results where about $50 \%$ of the $\sigma$-convergence was found to be euro zone-specific. This difference between rates and margins strongly suggests that the driving forces of retail rate convergence 
were of macro-economic rather than micro-economic nature. However, the $\beta$-convergence results for euro-zone mortgage margins reveal a convergence process that is present over the whole investigation period and is even increasing in the EMU period. However, non-euro zone $\beta$-convergence is about twice as high. Thus compared to a global benchmark, euro-zone mortgage margins exhibit relatively less $\beta$-convergence (rather than more as might be concluded when looking at mortgage rates). ${ }^{13}$ This result might therefore indicate that convergence of margins is not so much driven by euro zone-specific processes such as measures to promote a single banking market, but by a global process of financial innovation and competition.

Regarding corporate loan margins, the results reveal euro zone-specific $\sigma$ - and $\beta$ convergence only in the pre-EMU period. In contrast to corporate loan rates, however, the estimated coefficients are far less negative, indicating less convergence. As such, part of the observed convergence in interest rate levels is again likely to be due to the elimination of exchange rate uncertainty and overall falling interest rate levels. This interpretation is confirmed when looking at Panel G of Figure 1 where margins appear to converge only slightly and then only for a subgroup of euro-zone countries. Note that after 1998, $\beta$ convergence could also be observed for the non-euro zone, while for $\sigma$-convergence even some divergence is visible. Since the graphical picture reveals the existence of a "convergence-club" for corporate loans, we also conducted the analysis for a restricted euro zone excluding Germany, Belgium and Ireland. Interestingly, for these countries, both corporate rates and corporate rate margin are continuing to converge under the single currency on both measures. $^{14}$

\footnotetext{
${ }^{13}$ The results for mortgage margins over money market rates differ. Whereas the estimated coefficients are the same for the euro zone, they are lower for the non-euro zone. This leads to the conclusions that $6 \%$ of the preEMU convergence and $25 \%$ of the EMU convergence is euro zone-specific.

${ }^{14}$ Details are listed in Tables A1 to A4 in the appendix.
} 
The results of our case study of the mortgage and short-term corporate loan markets thus indicate that most of the convergence took place before 1999. The comparison of rates and margins strongly suggests that the driving forces of integration were macro-economic factors such as the integration of money markets which were taking place in anticipation of the single currency. Simple euro zone-only results of $\sigma$ - and $\beta$-convergence might indeed paint the picture of convergence - but the more appropriate DD analysis reveals that only a fraction of this convergence is euro zone-specific. Further convergence of margins did not occur under the single currency, and if it occurred - such as $\beta$-convergence of mortgage margins - it may rather be attributed to a change in the global competitive environment. Thus, the results from our DD-analysis are somewhat in line with the evidence from quantity-based measures of integration, which paint a more pessimistic picture of the state of European retail banking market integration than simple convergence studies. However, we could also document the existence of a "convergence-club" subgroup in the corporate loan market. This particular phenomenon suggests that further in-depth studies of determinants of convergence might be needed.

\section{Cointegration of retail banking market prices}

Retail banking markets may resist full convergence due to differences in national default risk, cultural influences in bank-client relationship, country-specific strategic bank behavior, the lack of cross-border lending, the lack of international bank mergers, and - at least up to January 1999 - differing monetary policy conditions. Consequently, one may not even expect the law of one price to hold in this market. Therefore, as first advocated in Kleimeier and Sander (2000) an alternative is to employ the concept of cointegration to analyze the state of market integration in retail banking when lending rates are tied together by a long-term equilibrium relationship that does not demand strict price- or product- 
equalization. Cointegration analyses consider a setting where time series of individual variables "can wander extensively and yet some pairs of series may be expected to move so they do not drift too far apart" (Engle and Granger 1987). This long-run cointegration relationship can be expressed on a bivariate level as

$\mathrm{y}_{\mathrm{t}}=\mathrm{c}_{1}+\mathrm{c}_{2} \mathrm{x}_{\mathrm{t}}+\mathrm{u}_{\mathrm{t}}$

where $\mathrm{y}$ indicates the retail bank rate in one country and $\mathrm{x}$ a benchmark-retail bank rate of another country or a regional average, while $\mathrm{u}_{\mathrm{t}}$ indicates the (estimated) error correction term. In the following we conduct cointegration analyses for regional and global mortgage and corporate lending rates which allows a direct comparison with the previously presented convergence results.

Looking first at the cointegration evidence obtained in our previous work (Kleimeier and Sander 2000 and 2003) over a relatively long period from 1985 to 2002 we can identify different phases of cointegration in the euro zone. Whereas it appears that the retail lending markets are weakly cointegrated before 1993, these links disappear in the mid-1990s. Around the introduction of the single currency, however, cointegration seems to appear again - at least in some retail markets, especially in the short-term corporate loan markets. Roughly speaking, cointegration analysis leads to a 'yes-no-yes' integration pattern over time to which the changes - in particular the ERM crises in 1992/93 - in the exchange rate regime appear to be most important. For the more recent period of the 1990s the role of the single currency is central to the analysis and is by most authors believed to introduce a shift in the structural relationships. Note, however, that as Kleimeier and Sander (2003) have shown for retail lending and deposit rates between 1995 and 2002, the break occurs in many retail markets well before January 1999, thus pointing towards an anticipation of the EMU.

In order to be directly able to compare our earlier convergence results to those of a cointegration analysis, we conduct cointegration analyses for all our euro zone and non-euro 
zone countries and for the same retail lending markets, the mortgage and the corporate loan market. While Kleimeier and Sander $(2000,2003)$ have investigated the cointegration relationship of an individual country against the remaining weighted average of the region, Schüler and Heinemann (2002) have argued in favor of investigating bilateral cointegration relationships. Since both analyses can provide complementary, though not contradictive results, we conduct here both types of analyses. In order to take the structural breaks and convergence processes explicitly into account, we estimate rolling cointegration regressions for five sub-periods of four years each. ${ }^{15}$ As such we obtain cointegration results for subperiods of January 1995 to December 1998, January 1996 to December 1999, etc.

Methodologically, the unit root characteristics of the individual, interest rate time series have to be established first. ${ }^{16}$ Then we test for cointegration based on equation (7) using the usual Durbin-Watson, Dickey-Fuller, and Augmented Dickey-Fuller tests. To establish the speed of adjustment of interest rates back to the long-run equilibrium, we also estimate an error-correction-model (ECM) in a two step procedure. We first estimate an unrestricted VAR model as

$$
\Delta \mathrm{y}_{\mathrm{t}}=\alpha_{0}+\chi_{0} \mathrm{y}_{\mathrm{t}-1}+\omega_{0} \mathrm{x}_{\mathrm{t}-1}+\sum_{\mathrm{j}=1}^{4} \chi_{\mathrm{j}} \Delta \mathrm{x}_{\mathrm{t}-\mathrm{j}}+\sum_{\mathrm{j}=1}^{4} \omega_{\mathrm{j}} \Delta \mathrm{y}_{\mathrm{t}-\mathrm{j}}+\varepsilon_{\mathrm{t}}
$$

Second, we then estimate the ECM as

$$
\Delta \mathrm{u}_{\mathrm{t}}=\alpha+\lambda \mathrm{u}_{\mathrm{t}-1}+\sum_{\mathrm{j}=1}^{4} \chi_{\mathrm{j}} \Delta \mathrm{x}_{\mathrm{t}-\mathrm{j}}+\sum_{\mathrm{j}=1}^{4} \omega_{\mathrm{j}} \Delta \mathrm{y}_{\mathrm{t}-\mathrm{j}}+\varepsilon_{\mathrm{t}}
$$

where $u_{t}$ is the estimated error of the cointegration regression and only the significant lags of $\Delta \mathrm{x}$ and $\Delta \mathrm{y}$ from equation (8) are included. The coefficient $\lambda$ indicates the estimated speed of adjustment.

\footnotetext{
${ }^{15}$ We are thereby consistent with other studies that estimate rolling cointegration regressions in order to illustrate the development of the cointegration over time (see e.g. Brada et al. 2005).

${ }^{16}$ See Table A5 of the appendix for detailed unit roots results.
} 
The results are summarized in Table $2 .{ }^{17}$ Both mortgage and corporate loan rates show the earlier mentioned yes-no-yes- pattern of bilateral cointegration within the euro zone. For mortgages, cointegration drops from 29 relationships in the pre-EMU sub-period from January 1995 to December 1998 (reflecting 32\% of the possible 90 bilateral relationships among the 10 national rates) to as few as 12 only to increase to 40 (44\%) during the last, EMU sub-period. Corporate loan rates show a similar pattern but reveal overall higher level of cointegration reaching $57(63 \%)$ bilateral cointegration relationships under the single currency. Cointegration relative to the euro-zone average reveals a similar pattern for corporate loan rates. During the last rolling period from 1999 to 2002 only 2 countries, Finland and Germany, are not cointegrated with the euro-zone average. On the other hand, very little cointegration can be found for mortgage rates. The cointegration relationships reported in Panel B of Table 2 refer mainly to French rates. ${ }^{18}$

\section{[insert Table 2 about here]}

In order to illustrate the current state of (co)integration in the retail banking markets, Table 3 highlights the cointegration and speed of adjustment results for the EMU period. Consider first bilateral cointegration: Within the euro-zone's mortgage markets, cointegration is mainly driven by Finland, France, Italy, and $\operatorname{Spain}^{19}$ whereas Germany does not seem to drive cointegration. The Irish mortgage market seems to be the one, which is least integrated with any other euro-zone market. As expected, cointegration is much more pronounced in corporate loan markets and speed of adjustment is higher here. Germany's limited role

\footnotetext{
${ }^{17}$ See Tables A6 and A7 of the appendix for details on the cointegration estimation.

${ }^{18}$ We also investigated a very early sample period from January 1990 to December 1993 . These results are, however, not fully comparable as no mortgage rates are available for Austria or Italy and as short-term corporate loan rates are missing for Austria. Overall, the yes-no-yes-shape observed in Table 2 appears to extend into the early period of the 1990s as well. In contrast to the end of the 1990s, however, mortgage rates show higher levels of cointegration than short-term corporate loan rates.
} 
remains: German corporate loan rates affect only Belgian rates. On the other hand, German rates do not respond to any other national rate. As such, this result corresponds with the earlier finding of a restricted "convergence-club" - however, here for both the corporate loan and mortgage market. We therefore also conducted a separate analysis with a restricted sample which shows that for corporate loan rates, there are cointegration relationships between $78 \%$ of all possible bilateral country-pairs within the restricted euro zone but only $44 \%$ within the outlier group of Belgium, Germany, and Ireland. Cointegration is also more complete with an average cointegration coefficient of -0.57 for the convergence club compared to the -0.31 for the outlier countries. For mortgage a similar picture emerges with $53 \%$ of all cointegration relationships and an average coefficient of -0.35 within the convergence club compared to $11 \%$ and -0.20 for the outlier countries. ${ }^{20}$

Consider now cointegration relative to the euro-zone average: The most interesting result is that UK lending rates are not cointegrated neither with individual euro-zone countries nor the euro-zone average but with the US rates, suggesting that the UK shows more similarities with the US than with the euro zone. In contrast, Swiss retail markets show some weak signs of cointegration with some euro-zone member countries. Somewhat puzzling are the results for Japanese rates. Note however, that the Japanese results could be driven by the poor unit root characteristics of the series.

\section{[insert Table 3 about here]}

\section{$\underline{4 .}$ Comparing convergence and cointegration measures of integration}

\footnotetext{
${ }^{19}$ That is, we find cointegration and significant speed of adjustment when these national rates are considered as the independent variable in the cointegration regression.

${ }^{20}$ Furthermore, our findings regarding Germany question the established approach of using a German rate as a benchmark or common factor. We are thereby in line with Dunne et al. (2002) who based on Granger causality analysis propose French and Italian rates as alternatives to German rates.
} 
When comparing the results of convergence and cointegration measures of integration the most important difference is found in the integration assessment of both, mortgage and corporate loan markets. Simple price convergence measures indicate that mortgage markets are the most integrated of all euro-zone retail lending markets. Looking at margins, however, shows that convergence did either not take place, was part of a more global process rather than a euro zone-specific one, or occurred only to a smaller euro-zone convergence club in the case of corporate loans. Cointegration analysis, however, additionally reveals that under the single currency both mortgage and corporate lending rates are now more closely related within this convergence club.

Consolidating these results reveals a weakness of the price-convergence measures in the context of euro-zone retail banking: Whereas the convergence of money market rates in anticipation of and under the single currency has provided a picture of overall convergence of nominal retail banking rates, the image of lack of integration that the quantity-based approaches clearly show is only consistently reflected in margin-convergence and cointegration analyses. Loan products as well as lender and borrower behavior remain different across the euro zone. In such an environment, simple price-convergence measures of integration are not well suited. It would indeed be surprising if in a market with different loan products or lending risks, interest rates were equal. In such a setting, cointegration measures of integration are more useful as they allow for these differences across national retail markets. On the other hand, cointegration measures will not be very useful when many structural changes are taking place over time. After the 1992/93 ERM crises and during the run-up towards the introduction of the single currency, some of the pre-1992 established cointegration relationships disappeared as exchange rate readjusted, inflation convergence occurred, and finally the fulfillment of the Maastricht criteria was envisaged. During this time, convergence measures reveals some progress in establishing a more uniform euro-zone 
retail banking market while cointegration analysis is not yet able to establish stable long-term relationships. However, in a structurally stable environment, cointegration measures are able to reveal important and detailed integration properties.

In sum, the comparison of convergence and cointegration measures of integration lead us to the following conclusions: Although price-convergence measures can provide an easy way to monitor some progress in integrating market, they may miss to benchmark against the rest of the world. Here exchange rate risks are present and convergence measures based on margins are therefore more appropriate to evaluate the impact of regional policies on banking market integration. Cointegration measures can - under stable conditions - help to identify a lack of integration on a bilateral and multilateral base. In contrast to Adam et al. (2002) we therefore advocate margin-convergence and cointegration measures to monitor the progress in European retail banking market integration and benchmark it against global retail banking developments.

\section{Retail banking integration by means of wholesale banking integration}

Retail banking still relies on geographic proximity to the customer and is characterized by substantial information asymmetries so that local banks often have a comparative advantage in screening local borrowers. This limits the emergence of an integrated retail banking market. Thus, the here documented lack of integration, i.e. the still segmented markets, provide diversification opportunities for the bank's loan portfolio as risk and return differ across national banking markets. Nevertheless, some integration is taking place and convergence of retail interest rates is documented. All in all, the above analyses suggest that the convergence and integration process observed in euro-zone retail markets is indeed largely driven by wholesale market integration. Two mechanisms are eventually the most relevant 
ones: the transmission of monetary impulses onto retail banking (lending) interest rates and the evolution towards a well-functioning euro-zone secondary market for loans.

Regarding the first mechanism the pass-through of interest rate changes onto retail interest rate is most relevant. Given the almost fully integrated money market in the euro zone it is clear that a full and homogeneous pass-through of interest rate changes onto retail rates would be sufficient to make the latter obey the law of one price within the euro zone. As such, pass-through studies provide information about banking market integration. Most of these studies find that the pass-through is typically the fastest and most homogeneous with respect to lending to corporations, while with respect to consumer lending the pass-through is most heterogeneous. These results are directly correlated with the evidence on (the lack of) retail market integration. In Sander and Kleimeier (2004) we particularly investigated the results of a pass-through study with respect to its integration implications. While the general conclusion is that the national pass-through processes are still highly divergent, we were nevertheless able to identify some convergence of the pass-through process with a particularly in the shortterm corporate loan market. Moreover, the single monetary policy regime, in particular a reduced money market rate volatility, appears to have been helpful in homogenizing monetary transmission. Furthermore, in a more recent study using Euribor interest rate futures to proxy interest rate expectations (Kleimeier and Sander 2005) we find that if monetary policy changes are anticipated this might also have a homogenizing impact on the pass-through. In sum, one can conclude that a tendency towards a more homogeneous transmission process within the euro zone goes some way in creating a more homogeneous retail banking market. The conduct of monetary policy plays an important role here, but national characteristic may resist full convergence for a longer period of time.

A second avenue towards more integration may be provided by the creation and fast development of a secondary market for loans. As suggested by the ECB (2002b) 
securitization might thus provide additional integration effects. Securitization in Europe currently amounts to 150 billion euro but is as such still underdeveloped in especially when compared to the US. ${ }^{21}$ Looking therefore at the effects that securitization had on the US retail banking markets and there in particular on the mortgage markets can provide valuable lessons. In the US, securitization first occurred in the mortgage market in the 1970s. A second wave took place in 1980s, which also marked the establishment of the government-sponsored agencies, GNMA, FNMA and FHLMC who took a leading role in the development. Private securitizing firms have joined the market and securitization has been steadily rising. Whereas on $5 \%$ of consumer loans were securitized in 1989 , this percentage had risen to $30 \%$ in 2000 . Major types of securitized loans now include student, manufactured housing, home equity, credit card, and auto loans. Securitization is, however, still most pronounced in the mortgage market where $46 \%$ of all mortgages were securitized in 2000 (Estrella 2002).

Two main types of benefits from mortgage securitization have been advocated for the US and could thus eventually be expected in Europe: First, benefits from the deepening of the mortgage market and second, benefits from the broadening of the mortgage market. Regarding the first benefit, it appears that increased securitization is correlated with lower interest rate spreads on mortgages. Kolari et al. (1998, p. 679) find that "a $10 \%$ increase in the level of securitization as a proportion of total mortgage origination reduces the yield spread on home mortgage loans by as much as 20 basis points in the long run". However, some authors question the causality pattern and argue that low spreads may have led to more securitization. $^{22}$ Regarding the second benefit, Katz (1997) argues that cross-sectional

\footnotetext{
${ }^{21}$ The legal and regulatory changes adopted during the 1990s, in particular the allowance of special purpose vehicles and financial vehicle corporations, were crucial in fostering the creation of many new financial instruments. This has contributed to the growth of securitization in particular in Belgium, Spain and Portugal. However, national patterns prevail and differences include the type of debt that is securitized (household versus corporate debt), the maturity of the created securities (short- versus long-term) and the relative quantities of securitized debt.

${ }^{22}$ See e.g. Estrella (2002) who argues on the base of a study by Heuson, Passmore, and Sparks (2001), that low mortgage rates cause more securitization. As such the negation correlation might not necessarily have to be interpreted as consumer benefits.
} 
dispersion in mortgage rates in the US is negatively related to securitization. Whereas regional mortgage rates differed by 100 basis points or more, they have become much more homogeneous. She believes that banks in the US use securitization to reduce their sensitivity to local economic shocks. Due to their superior knowledge of market conditions, property values, and creditworthiness of borrowers, banks still concentrate their loan origination in particular regions or industries. These loans, however, do not stay on the balance sheet but are securitized and sold to banks anywhere in the country. As a consequence "[m]oney can flow from regions with idle deposits to those with excess loan demand, and thus mortgage rates are more similar across the country."

This US evidence thus implies that increasing securitization in the euro zone may help to generate the so far still elusive integration benefits and could eventually lead to further convergence of retail lending rates in a market where banks and bank lending can remain domestic in nature. However, as noted before, the first effect is disputed and might require further research. Moreover, eventual benefits would have to be weighted against potential costs. For example, securitization is believed to affect the stability of the banking sector. While on the one hand, stability is increased as banks can hold more diversified portfolios and are less dependent on the local economy (Katz 1997), stability is on the other hand decreased if securitization is used for regulatory capital arbitrage (Jones 2000) or leads to insufficient monitoring of borrowers. ${ }^{23}$

Finally, securitization can have an impact on the effectiveness of monetary policy transmission, in particular with respect to the pass-through mechanism. Estrella (2002) finds a stronger effect of monetary policy on mortgage rates due to increased securitization. However, as securitization allows banks to continue their lending activities even under monetary tightening as securitized loans do not need to be funded by deposits, monetary

\footnotetext{
${ }^{23}$ Lisieri et al. (2001) report evidence for a free-rider problem in UK mortgage markets in the 1980 s.
} 
policy seems to have very little effect on output. Kuttner (2000) provides empirical evidence that banks do indeed use securitization as a monetary policy buffer. He finds that in their reaction to monetary policy, securitization volume moves in the opposite direction to bank loan volume. Thus, consumers might benefit as credit crunches are avoided.

\section{$\underline{\text { 6. Conclusions }}$}

Twelve years after the launch of the single market project and five years after the introduction of the single currency the evolving empirical evidence supports the view of still fragmented retail markets with strong national characteristics. The evidence of convergence of retail banking markets is largely a result of integrating money and bonds markets in anticipation of the single currency. Important structural changes in the euro-zone retail banking markets can indeed be identified but should largely be attributed to the single currency and the impact of the single monetary policy. As such, three major conclusions emerge. First, without further changes in regulation and/or the competitive environment the convergence process might now slow down considerably or even come to a halt. Secondly, obtaining further integration effects and thus convergence may only be possible by more integration of wholesale markets through secondary loan markets and securitization. However, regulators have to judge whether the potential benefits of such activities outweigh the potential risks and cost. Finally, retail-banking activities tend to remain localized. As such, monitoring and eventually regulating competition in these markets will remain a major concern for policy makers.

\section{$\underline{\text { Appendix }}$}

[insert tables A1 to A7 about here] 


\section{$\underline{\text { References }}$}

Adam, K., Jappelli, T., Menichini, A., Padula, M., Pagano, M. 2002. Analyse, compare, and apply alternative indicators and monitoring methodologies to measure the evolution of capital market integration in the European Union. Working paper, Centre for Studies in Economics and Finance (CSEF), University of Salerno.

Baele, L., Ferrando, A., Hördahl, P., Krylova, E., Monnet, C., 2004. Measuring financial integration in the euro area, European Central Bank Occasional Paper Series No.14, April, Frankfurt.

Brada, J.C., Kutan, A.M., Zhou, S., 2005. Real and monetary convergence within the European Union and between the European Union and candidate countries: A rolling cointegration approach. Journal of Banking and Finance 29, 249-270.

Cabral, I., Dierick, F., Vesala, J., 2002. Banking integration in the euro area. ECB Occasional Paper No. 6, Frankfurt.

Centeno M., Mello, A.S., 1999. How integrated are the money market and the bank loans market within the European Union? Journal of International Money and Finance 18, 75106.

Corvoisier, S., Gropp, R., 2002. Bank concentration and retail interest rates. Journal of Banking and Finance (26), 2155-2189.

De Bondt, G., 2002. Retail bank interest pass-through: new evidence at the euro area level. ECB Working Paper 136, Frankfurt.

De Bondt, G., Mojon, B., Valla, N., 2002. Interest rate setting by universal banks and the monetary policy transmission mechanism in the euro area. Mimeo.

Dunne, P., Moore, M., Portes, R., 2002. Defining benchmark status: an application using euro area bonds. CEPR Working Paper No. 3490, London. 
Economic and Financial Committee, 2002. Report by the Economic and Financial Committee (ETC) on EU financial integration. European Commission, Directorate-General for Economic and Financial Affairs. Economic Papers No. 171, Brussels.

Engle, R.F., Granger, C.W.J., 1987. Cointegration and error correction: representation, estimation, and testing. Econometrica 55, 251-276.

Estrella, A., 2002. Securitization and the efficacy of monetary policy. Federal Reserve Bank of New York Economic Policy Review, May, 243-255.

European Central Bank, 2003. Manual on MFI interest rate statistics. Regulation ECB/2001/18. European Central Bank, Frankfurt, October.

European Commission (2004). Financial Integration Monitor 2004. Background Document. Internal Market DG, Brussels 28 April 2004.

Freixas, X., 2003. European banking integration: where do we stand? Deutsche Bank Lecture presented on Thursday, 5 June, in the Saïd Business School.

Heinemann, F., Schüler, M., 2003. Integration benefits on EU retail credit markets - Evidence from interest rate pass-through. In: Cecchini, P., The incomplete European market for financial services. Springer Verlag, Berlin, pp. 105-129.

Heuson, A., Passmore, W., Sparks, R., 2001. Credit scoring and mortgage securitization: implications for mortgage rates and credit availability. Journal of Real Estate Finance and Economics 23, 337-63.

Jones, D., 2000. Emerging problems with the Basel Capital Accord: regulatory capital arbitrage and related issues. Journal of Banking and Finance 24, 35-58.

Katz, J., 1997. Getting Secure. Federal Reserve Bank of Boston Regional Review 3, Summer, $13-7$. 
Kleimeier, S., Sander, H, 2005. Expected versus unexpected monetary policy impulses and interest rate pass-through in euro-zone retail banking, forthcoming in Journal of Banking and Finance.

Kleimeier, S., Sander, H., 2003. European financial market integration: evidence on the emergence of a single eurozone retail banking market. Research in Banking and Finance $3,13-91$.

Kleimeier, S., Sander, H., 2000. Regionalisation versus globalisation in European financial market integration: evidence from co-integration analysis. Journal of Banking and Finance 24, 1005-1043.

Kolari, J.W., Fraser, D.R., Anari, A., 1998. The effects of securitization on mortgage market yields: a cointegration analysis. Real Estate Economics 26(4), 677-693.

Kuttner, K., 2001. Monetary policy surprises and interest rates: Evidence from the Fed funds futures market. Journal of Monetary Economics 47(3), 523-544.

Kuttner, K., 2000. Securitization and monetary policy. Unpublished paper, Federal Reserve Bank of New York.

Lisieri, C., Ward, C., Palmer, S., 2001. Financial innovation in property markets implications for the City of London. Report for The Corporation of London in association with the RICS Research Foundation.

Manna, M., 2004. Developing statistical indicators of the integration of the euro area banking system. ECB Working Paper No. 300, Frankfurt.

Mojon, B., 2000. Financial structure and the interest channel of the ECB monetary policy. ECB Working Paper No. 40, Frankfurt.

Padoa-Schioppa, T., 2000. Is a euroland banking system already emerging? Lecture at the Société Universitaire Européenne de Recherches Financières, Vienna 29 April, European Central Bank (www.ecb.int/key/00/sp000429.htm). 
Sander, H., Kleimeier, S., 2004. Convergence in euro-zone retail banking? what interest rate pass-through tells us about monetary policy transmission, competition and integration. Journal of International Money and Finance 23, 461-492.

Schüler, M., Heinemann, F., 2002. How integrated are the European retail financial markets? a cointegration analysis. Deutsche Bank Research Notes No. 3, Deutsche Bank, Frankfurt.

Slaughter, M.J., 2001. Trade liberalization and per capita income convergence: a difference-indifferences analysis. Journal of International Economics 55, 203-228.

Toolsema, L.A., Sturm, J.-E., de Haan, J., 2002. Convergence of pass-through from money market to lending rates in EMU countries: new evidence. University of Groningen, February, mimeo. 


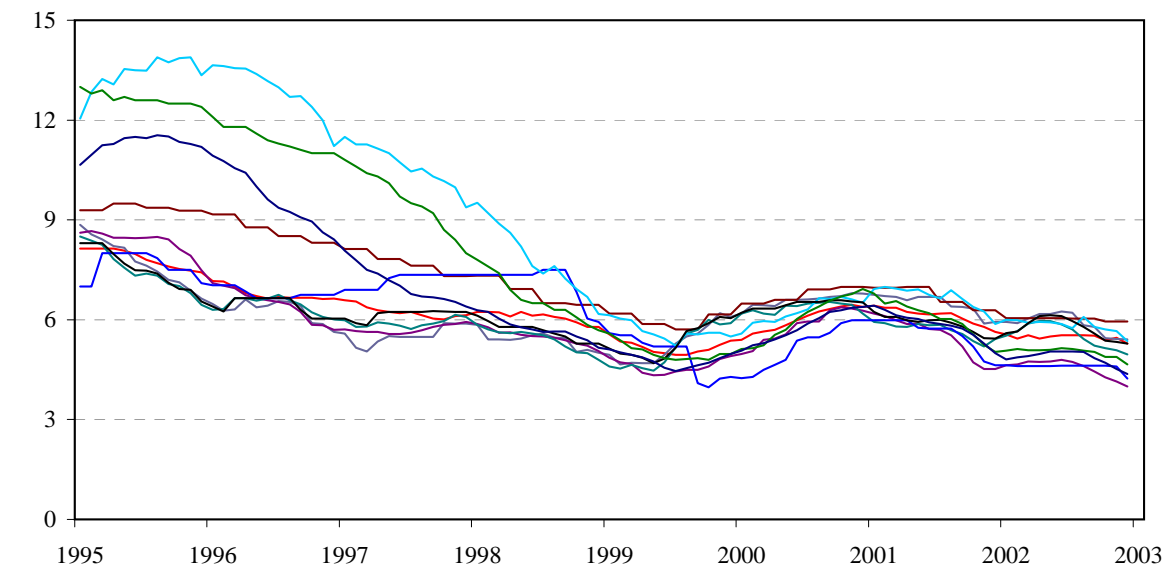

Panel C: Eurozone short-term corporate loan rates

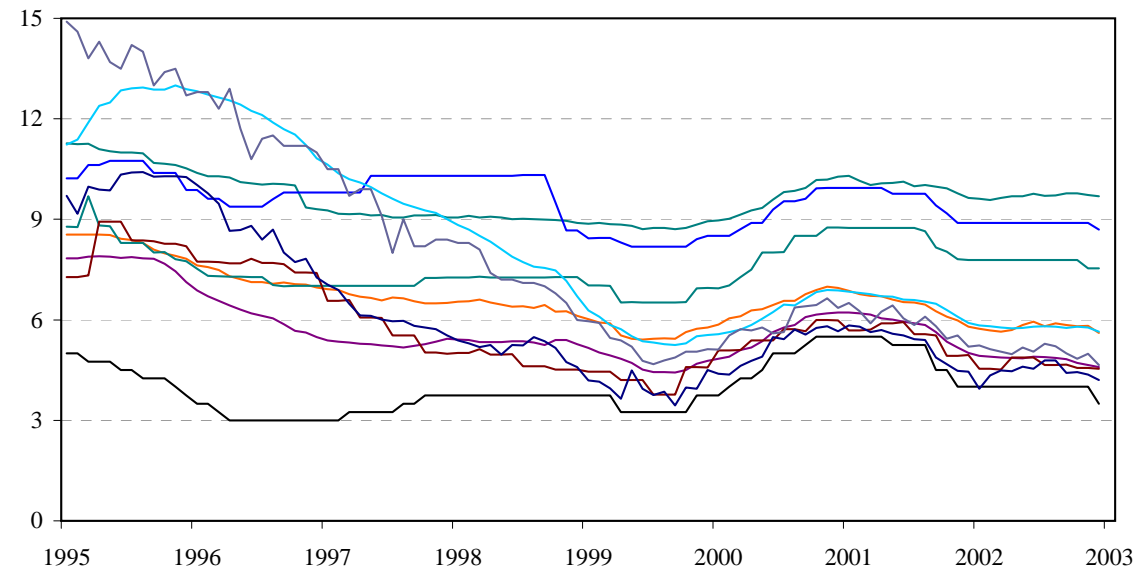

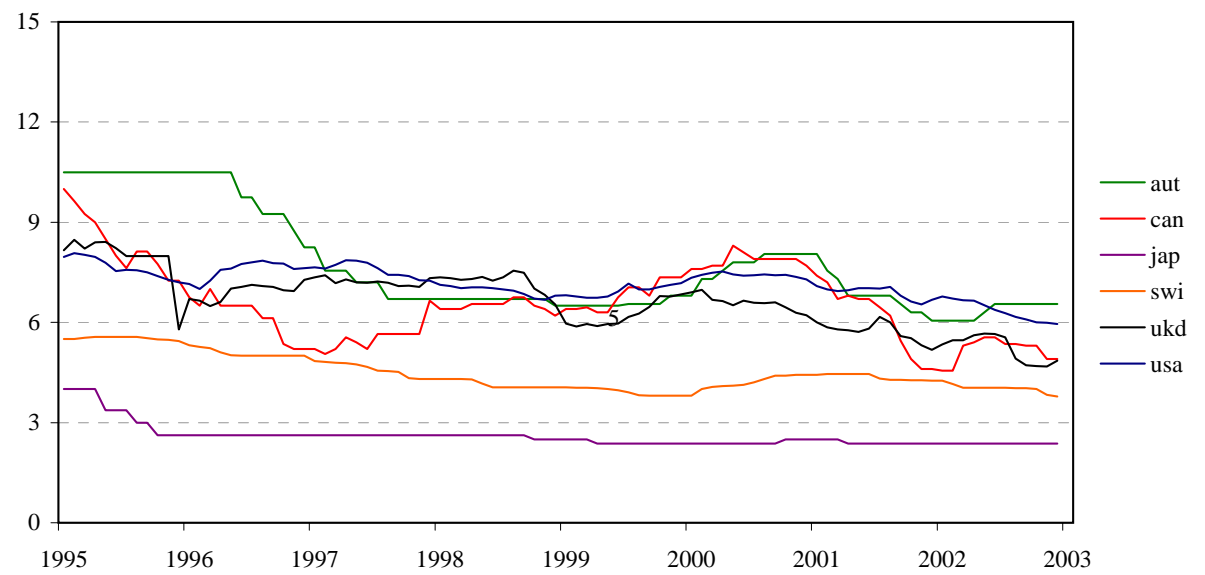

Panel D: Non-eurozone short-term corporate loan rates

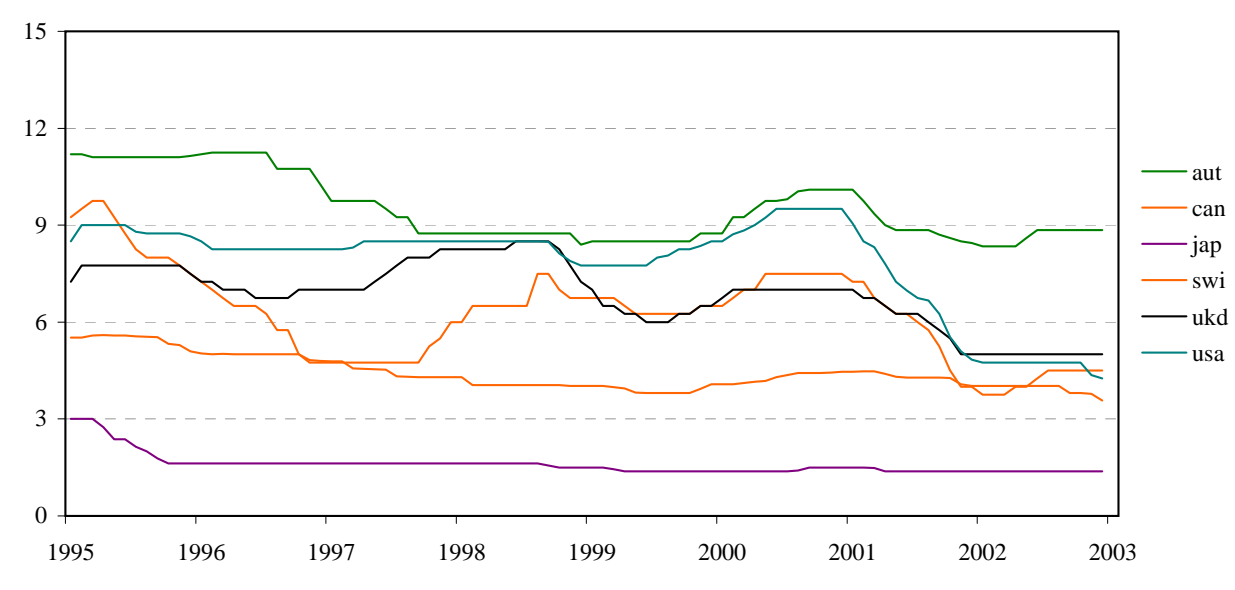




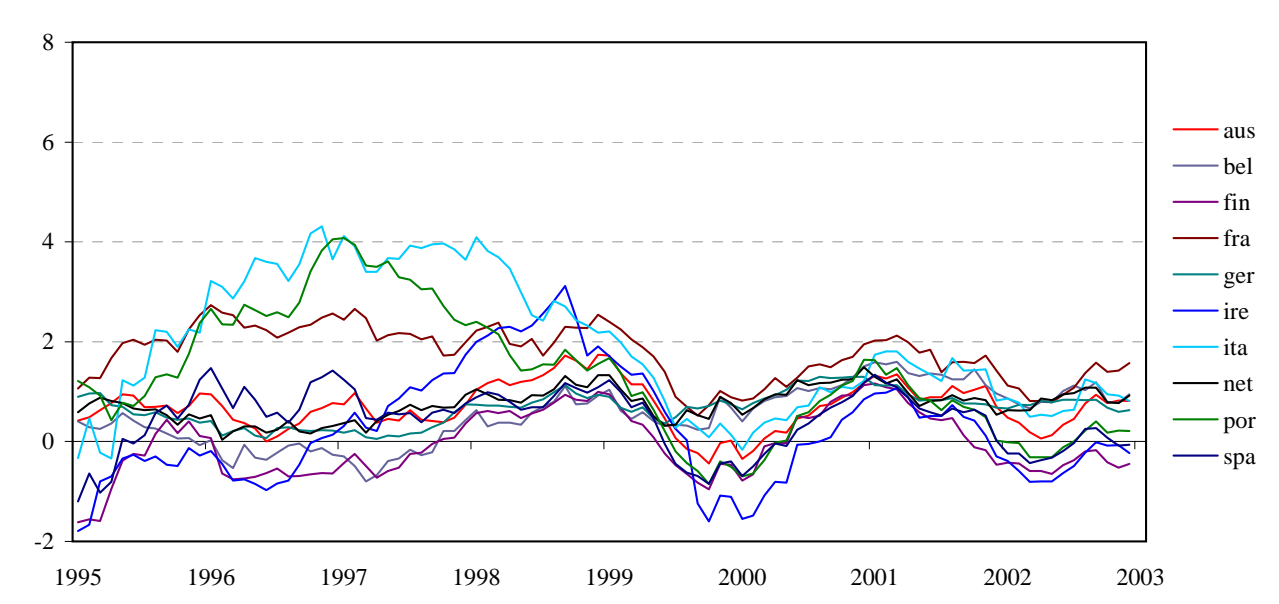

Panel G: Eurozone short-term corporate loan rate margins over 3-months money market rate
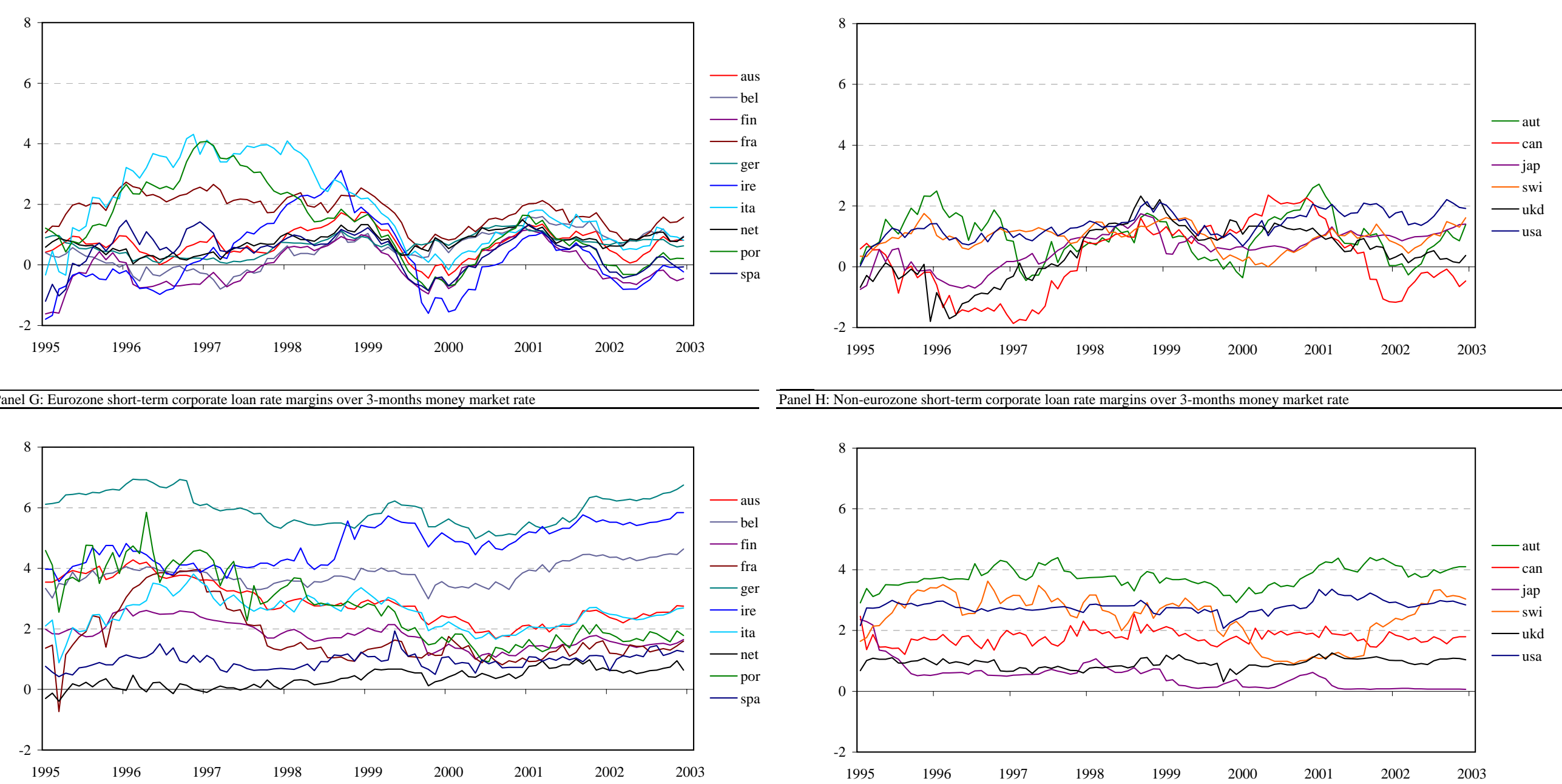

Panel H: Non-eurozone short-term corporate loan rate margins over 3-months money market rate

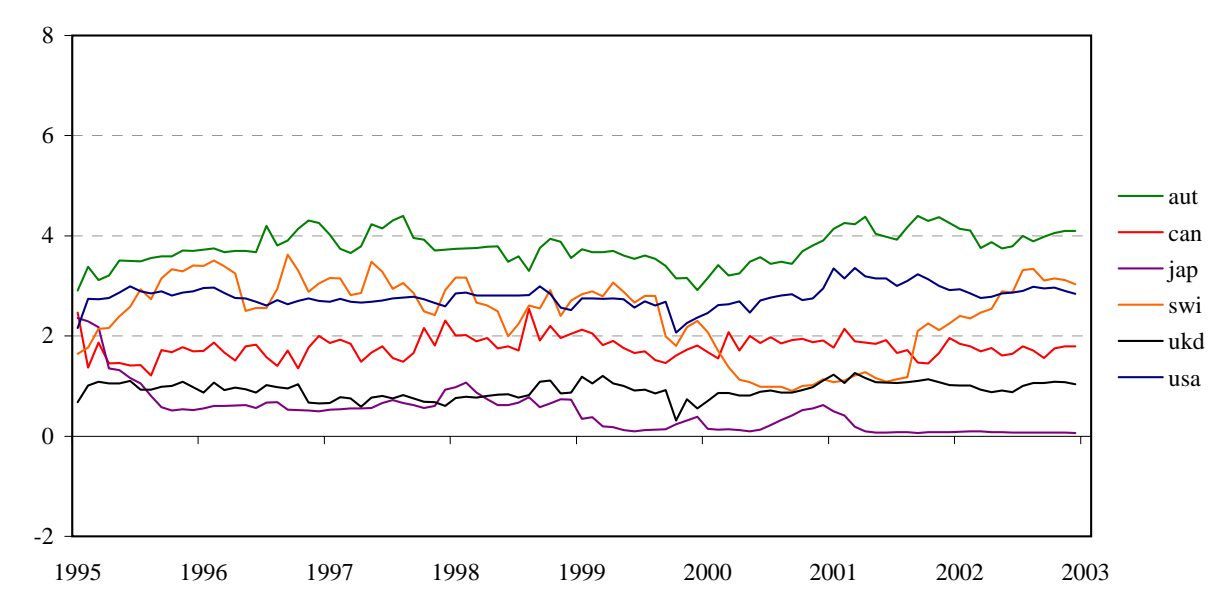

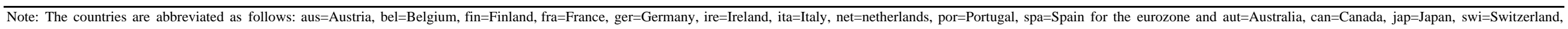
ukd=United Kingdom, usa=United States of America for the non-eurozone. On the $x$-achsis, the numbers are placed such that they indicate the beginning of the year. 
Table 1: Differences in regional convergence based on differences-in-differences analysis

\begin{tabular}{|c|c|c|c|c|}
\hline \multicolumn{5}{|l|}{ Panel A: Mortgage rates } \\
\hline \multirow[b]{2}{*}{ convergence coefficient for region } & \multicolumn{2}{|c|}{$\sigma$-convergence } & \multicolumn{2}{|c|}{$\beta$-convergence } \\
\hline & $\begin{array}{r}\text { pre-EMU } \\
\text { period }\end{array}$ & $\begin{array}{r}\text { EMU } \\
\text { period }\end{array}$ & $\begin{array}{r}\text { pre-EMU } \\
\text { period }\end{array}$ & $\begin{array}{r}\text { EMU } \\
\text { period } \\
\end{array}$ \\
\hline eurozone & $-0.041^{*}$ & 0.000 & $-0.257^{*}$ & $-0.504^{*}$ \\
\hline non-eurozone & $-0.020 *$ & $-0.012 *$ & $-0.170 *$ & $-0.108 *$ \\
\hline F-test for differences across coefficients & F-test & signif & F-test & signif \\
\hline eurozone versus non-eurzone pre-EMU convergence & 40.173 & $0.0 \%$ & 10.909 & $0.0 \%$ \\
\hline eurozone versus non-eurzone EMU convergence & 13.140 & $0.0 \%$ & 28.507 & $0.0 \%$ \\
\hline pre-EMU versus EMU eurozone convergence & 152.191 & $0.0 \%$ & 11.834 & $0.0 \%$ \\
\hline pre-EMU versus EMU non-eurozone convergence & 5.633 & $1.9 \%$ & 3.690 & $5.5 \%$ \\
\hline \multicolumn{5}{|l|}{ Panel B: Short-term corporate loan rates } \\
\hline & \multicolumn{2}{|c|}{$\sigma$-convergence } & \multicolumn{2}{|c|}{$\beta$-convergence } \\
\hline convergence coefficient for region & $\begin{array}{r}\text { pre-EMU } \\
\text { period }\end{array}$ & $\begin{array}{r}\text { EMU } \\
\text { period } \\
\end{array}$ & $\begin{array}{r}\text { pre-EMU } \\
\text { period }\end{array}$ & $\begin{array}{r}\text { EMU } \\
\text { period } \\
\end{array}$ \\
\hline eurozone & $-0.021^{*}$ & $0.006^{*}$ & $-0.155^{*}$ & 0.026 \\
\hline non-eurozone & $-0.007 *$ & $-0.012 *$ & $-0.074 *$ & $-0.087 *$ \\
\hline F-test for differences across coefficients & F-test & signif & F-test & signif \\
\hline eurozone versus non-eurzone pre-EMU convergence & 30.070 & $0.0 \%$ & 12.213 & $0.0 \%$ \\
\hline eurozone versus non-eurzone EMU convergence & 50.618 & $0.0 \%$ & 15.330 & $0.0 \%$ \\
\hline pre-EMU versus EMU eurozone convergence & 114.961 & $0.0 \%$ & 44.973 & $0.0 \%$ \\
\hline pre-EMU versus EMU non-eurozone convergence & 3.520 & $6.2 \%$ & 0.277 & $59.8 \%$ \\
\hline \multicolumn{5}{|c|}{ Panel C: Mortgage rate margins over 10-year government bond yield } \\
\hline & \multicolumn{2}{|c|}{$\sigma$-convergence } & \multicolumn{2}{|c|}{$\beta$-convergence } \\
\hline convergence coefficient for region & $\begin{array}{r}\text { pre-EMU } \\
\text { period } \\
\end{array}$ & $\begin{array}{r}\text { EMU } \\
\text { period } \\
\end{array}$ & $\begin{array}{r}\text { pre-EMU } \\
\text { period }\end{array}$ & $\begin{array}{r}\text { EMU } \\
\text { period } \\
\end{array}$ \\
\hline eurozone & -0.003 & 0.000 & $-0.251 *$ & $-0.471^{*}$ \\
\hline non-eurozone & $-0.145^{*}$ & $0.010^{*}$ & $-0.490 *$ & $-0.795 *$ \\
\hline F-test for differences across coefficients & F-test & signif & F-test & signif \\
\hline eurozone versus non-eurzone pre-EMU convergence & 8.970 & $0.3 \%$ & 17.665 & $0.0 \%$ \\
\hline eurozone versus non-eurzone EMU convergence & 6.081 & $1.5 \%$ & 9.302 & $0.2 \%$ \\
\hline pre-EMU versus EMU eurozone convergence & 0.487 & $48.6 \%$ & 8.900 & $0.3 \%$ \\
\hline pre-EMU versus EMU non-eurozone convergence & 37.928 & $0.0 \%$ & 10.258 & $0.1 \%$ \\
\hline \multicolumn{5}{|c|}{ Panel D: Short-term corporate loan rate margins over 3-months money market rate } \\
\hline & \multicolumn{2}{|c|}{$\sigma$-convergence } & \multicolumn{2}{|c|}{$\beta$-convergence } \\
\hline convergence coefficient for region & $\begin{array}{r}\text { pre-EMU } \\
\text { period } \\
\end{array}$ & $\begin{array}{r}\text { EMU } \\
\text { period } \\
\end{array}$ & $\begin{array}{r}\text { pre-EMU } \\
\text { period }\end{array}$ & $\begin{array}{r}\text { EMU } \\
\text { period } \\
\end{array}$ \\
\hline eurozone & $-0.008 *$ & $0.006^{*}$ & $-0.109 *$ & 0.026 \\
\hline non-eurozone & $0.003^{*}$ & $0.006^{*}$ & -0.025 & $-0.101^{*}$ \\
\hline F-test for differences across coefficients & F-test & signif & F-test & signif \\
\hline eurozone versus non-eurzone pre-EMU convergence & 66.001 & $0.0 \%$ & 7.220 & $0.7 \%$ \\
\hline eurozone versus non-eurzone EMU convergence & 0.297 & $58.6 \%$ & 14.639 & $0.0 \%$ \\
\hline pre-EMU versus EMU eurozone convergence & 109.816 & $0.0 \%$ & 47.767 & $0.0 \%$ \\
\hline pre-EMU versus EMU non-eurozone convergence & 3.275 & $7.2 \%$ & 3.408 & $6.5 \%$ \\
\hline
\end{tabular}

Notes: Based on F-tests, ${ }^{*}, * *$, and $* * *$ indicate that the convergence estimates are significantly different from zero at $1 \%, 5 \%$, and $10 \%$ significance level, respectively. Pre-EMU period refers to the time from 1.1.1995 to 31.12.1998 whereas EMU period refers to 1.1.1999 to 31.12.2002. The eurozone includes the countries Austria, Belgium, Finland, France, Germany, Ireland, Italy, the Netherlands, Portugal, and Spain. The non-eurozone includes the countries Australia, Canada, Japan, Switzerland, the United Kingdom, and the USA. The convergence coefficients are calculated based on equations (5) and (6). In particular, $\sigma$-convergence in the non-euro zone in the pre-EMU period is given by $b_{1}$. Convergence in the non-euro zone in the EMU period is given by $\left(b_{1}+b_{2}\right)$ convergence in the euro zone in the pre-EMU period is given by $\left(b_{1}+b_{3}\right)$, and finally convergence in the euro zone in the EMU period is given by $\left(b_{1}+b_{2}+b_{3}+b_{4}\right)$. In a corresponding manner ß-convergence can be calculated using $\mathrm{d}_{1}$ to $\mathrm{d}_{4}$. 


\section{Panel A: Bilateral cointegration}

\begin{tabular}{lllllllllll}
$1995-1998$ & $1996-1999$ & $1997-2000$ & $1998-2001$ & $1999-2002$ & & $1995-1998$ & $1996-1999$ & $1997-2000$ & $1998-2001$ & $1999-2002$ \\
\hline
\end{tabular}

$\quad 13 \%-13 \%-22 \%-44 \%$

$22 \% \quad 8 \%$

$37 \% \quad 17 \%$

$\mathbf{- 0 . 1 7}-\mathbf{0 . 3 1}$

$-0.24-0.17$

$\begin{array}{ll}-0.24 & -0.17 \\ -0.31 & -0.15\end{array}$

number of cointegration relationships

among eurozone countries

eurozone vs non-eurozone countrie

among non-eurozone countries

$\%$ of cointegration relationships

\section{among eurozone countries}

eurozone vs non-eurozone countries

among non-eurozone countries

among eurozone countries

eurozone vs non-eurozone countries

among non-eurozone countries

average speed of adjustment

29
26
11

Panel B: Cointegration relative to eurozon

number of cointegration relationships

\section{eurozone countries}

non-eurozone countries

$\%$ of cointegration relationships

eurozone countries

non-eurozone countries

\section{eurozone countries}

non-eurozone countries

$12 \quad 12$

9

averas

$\begin{array}{rrrrrrrrrr}\mathbf{2} & \mathbf{1} & \mathbf{1} & \mathbf{2} & \mathbf{2} & \mathbf{5} & \mathbf{2} & \mathbf{1} & \mathbf{3} & \mathbf{8} \\ 2 & 0 & 0 & 0 & 1 & 1 & 1 & 1 & 1 & 1 \\ \mathbf{2 0 \%} & \mathbf{1 0 \%} & \mathbf{1 0 \%} & \mathbf{2 0 \%} & \mathbf{2 0 \%} & \mathbf{5 0 \%} & \mathbf{2 0 \%} & \mathbf{1 0 \%} & \mathbf{3 0 \%} & \mathbf{8 0 \%} \\ 33 \% & 0 \% & 0 \% & 0 \% & 17 \% & 17 \% & 17 \% & 17 \% & 17 \% & 17 \% \\ & & & & & & & & & \\ -\mathbf{0 . 3 0} & \mathbf{- 0 . 3 2} & \mathbf{- 0 . 5 7} & \mathbf{- 0 . 2 7} & -\mathbf{0 . 3 3} & \mathbf{- 0 . 2 4} & \mathbf{- 0 . 3 6} & \mathbf{- 0 . 7 6} & \mathbf{- 0 . 3 0} & \mathbf{- 0 . 4 0} \\ -0.23 & & & & -0.25 & -0.13 & -0.23 & -0.30 & -0.44 & -0.20\end{array}$
calculate the average speed of adjustment, observations are deleted when no cointegration exists and observations are set to zero when cointegration exists but the coefficient is insignificant. 


\begin{tabular}{|c|c|c|c|c|c|c|c|c|c|c|c|c|c|c|c|c|c|}
\hline & 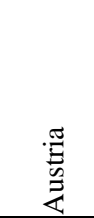 & $\begin{array}{l}\text { 慁 } \\
\text { 总 } \\
\text { 品 }\end{array}$ & 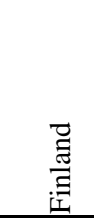 & 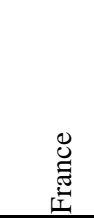 & 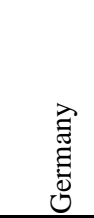 & 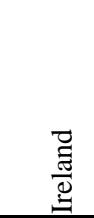 & 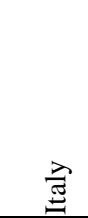 & 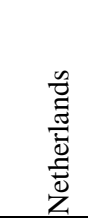 & 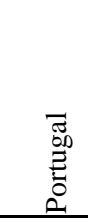 & $\begin{array}{l}\text { 寻 } \\
\text { की }\end{array}$ & 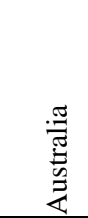 & $\begin{array}{l}\frac{\pi}{\pi} \\
\mathbb{J} \\
\tilde{U}\end{array}$ & $\begin{array}{l}\text { శ్ } \\
\text { 心్తి }\end{array}$ & 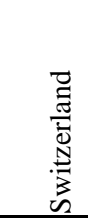 & 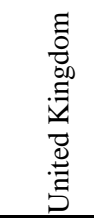 & 岕 & 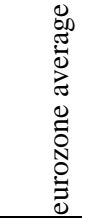 \\
\hline \multicolumn{18}{|c|}{ Panel A: Mortgage rates } \\
\hline Austria & & & & -0.36 & & & -0.50 & & & & & & & & -0.08 & & \\
\hline Belgium & -0.34 & & -0.28 & -0.37 & & -0.20 & -0.43 & & -0.31 & -0.37 & -0.17 & & & -0.32 & & & -0.36 \\
\hline Finland & & & & -0.67 & & & & & & -0.21 & & & & & & & \\
\hline France & -0.43 & -0.22 & -0.45 & & & & -0.38 & & -0.43 & -0.52 & & & & & & & -0.30 \\
\hline Germany & & & -0.31 & -0.30 & & & & -0.41 & & & & & & & & & \\
\hline Ireland & & & & & & & -0.33 & & & & & & & -0.34 & & & \\
\hline Italy & -0.53 & & -0.20 & -0.41 & & -0.29 & & & -0.34 & -0.52 & & & & -0.48 & & & \\
\hline Netherlands & & & -0.25 & -0.36 & -0.39 & & -0.22 & & & -0.28 & & & & & & & \\
\hline Portugal & & & 0.00 & -0.34 & & & -0.28 & & & -0.34 & & & & & & & \\
\hline Spain & & & 0.00 & -0.44 & & & -0.45 & & -0.34 & & & & & & & & \\
\hline \multicolumn{18}{|l|}{ Australia } \\
\hline \multicolumn{18}{|l|}{ Canada } \\
\hline Japan & -0.27 & -0.20 & -0.27 & -0.29 & -0.19 & -0.32 & -0.27 & -0.20 & -0.34 & -0.31 & 0.00 & -0.22 & & -0.28 & -0.20 & -0.21 & -0.25 \\
\hline Switzerland & -0.20 & & & & & & -0.45 & & & & & & & & & & \\
\hline United Kingdom & & & & & & & & & & & & & & & & -0.21 & \\
\hline \multicolumn{18}{|l|}{ USA } \\
\hline \multicolumn{18}{|c|}{ Panel B: Short-term corporate loan rates } \\
\hline Austria & & & -0.30 & -0.51 & & & & -0.49 & -0.73 & -0.54 & & & & 0.00 & & & -0.25 \\
\hline Belgium & & & & -0.29 & -0.30 & -0.56 & & & & -0.40 & & & & & & & -0.48 \\
\hline Finland & -0.32 & & & -0.42 & & & & -0.53 & -0.67 & -0.50 & & & & 0.00 & & & \\
\hline France & -0.58 & -0.41 & -0.57 & & & -0.42 & -0.39 & -0.75 & -0.69 & -0.69 & & & & -0.51 & & & -0.44 \\
\hline Germany & & 0.00 & & & & & & & & & & & & & & & \\
\hline Ireland & & -0.37 & & -0.37 & & & -0.45 & -0.26 & & -0.51 & & & & & & & -0.54 \\
\hline Italy & & & & -0.28 & & -0.30 & & -0.39 & -0.42 & -0.48 & & & & & & & 0.00 \\
\hline Netherlands & -0.45 & 0.00 & -0.64 & -0.72 & & -0.26 & -0.52 & & -0.60 & -0.86 & & & & -0.30 & & & -0.56 \\
\hline Portugal & -0.79 & & -0.78 & -0.63 & & -0.36 & -0.39 & -0.63 & 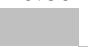 & -0.42 & & & -0.21 & -0.63 & & & -0.29 \\
\hline Spain & -0.74 & -0.46 & -0.47 & -0.70 & 0.00 & -0.70 & -0.75 & -0.94 & -0.49 & & -0.28 & & & -0.33 & & & -0.67 \\
\hline \multicolumn{18}{|l|}{ Australia } \\
\hline Canada & & & & & & & & & & & & & & & -0.27 & & \\
\hline Japan & -0.30 & -0.22 & -0.28 & -0.26 & -0.21 & -0.20 & -0.27 & -0.28 & -0.31 & -0.29 & -0.22 & -0.22 & & -0.26 & -0.19 & -0.23 & -0.20 \\
\hline Switzerland & 0.00 & & -0.40 & -0.39 & & & & 0.00 & -0.69 & 0.00 & & & & & & & \\
\hline United Kingdom & & & & & & & & & & & & -0.30 & & & & -0.21 & \\
\hline USA & & & & & & & & & & & & & & & -0.21 & & \\
\hline
\end{tabular}


Table A1: Data description

\begin{tabular}{|c|c|c|c|c|}
\hline Country & Source $^{1}$ & Description $^{2}$ & Code & Weight $^{3}$ \\
\hline \multicolumn{5}{|c|}{ Panel A: Mortgage rates } \\
\hline Austria & ECB & Loans for house purchase at variable rate & N2 & 3.01 \\
\hline Belgium & ECB & Mortgage loans with amortisation & N2 & 3.90 \\
\hline Finland & ECB & Housing loans to households & N2 & 1.69 \\
\hline France & DS & FR housing loans - fixed-rate loans curn & FRLPHSE\% & 20.99 \\
\hline Germany & ECB & Mortgage loans (5 years fixed rate) & N2 & 30.55 \\
\hline Ireland & ECB & Variable mortgage lending to households & N2 & 1.14 \\
\hline Italy & ECB & Loans over 18 months to households & N2 & 20.15 \\
\hline Netherlands & ECB & Mortgage loans from credit institutions & N2 & 5.74 \\
\hline Portugal & ECB & Loans for house purchase: loans and advances to private individuals with maturity over 5 years & $\mathrm{N} 2$ & 2.39 \\
\hline Spain & ECB & Mortgage loans for house purchase over 3 years & N2 & 10.44 \\
\hline Australia & $\mathrm{CB}$ & F05 indicator lending rates - Housing Loan - Variable rate - standard bank loans & FILRHLVBS & 0.00 \\
\hline Canada & DS & CN interest rates: 1 year mortgage rate,CH.bank (end month) & CNB14050 & 0.00 \\
\hline Japan & DS & JP city banks housing loans floating rate & JPFHOUSE & 0.00 \\
\hline Switzerland & DS & SW mortgage rate - new mortgages (12 cantonal banks) NADJ & SWKY4013F & 0.00 \\
\hline United Kingdom & ECB & Fixed rate mortgage loans, 2 years & $\mathrm{N} 2.2$ & 0.00 \\
\hline USA & DS & US new home mortgages - contract interest rate & USYMICN1 & 0.00 \\
\hline \multicolumn{5}{|c|}{ Panel B: Short-term corporate loan rates } \\
\hline Austria & ECB & Loans to enterprises & N4 & 3.01 \\
\hline Belgium & ECB & Bank advances in current account for professional use & N4-2 & 3.90 \\
\hline Finland & IFS & Average bank lending rate - Central bank: bulletin & $60 \mathrm{~b}$ & 1.69 \\
\hline France & ECB & Discount, overdrafts and other short-term loans & N4 & 20.99 \\
\hline Germany & IFS & Current acct. credit to 1 mill - Central bank: bulletin & $60 \mathrm{~b}$ & 30.55 \\
\hline Ireland & ECB & Overdrafts and term loans up to 1 year - AA rate/ lending to firms & N4 & 1.14 \\
\hline Italy & ECB & Interest on loans up to 18 months - all customers & N4-1 & 20.15 \\
\hline Netherlands & ECB & Bank base rate, enterprises & N4 & 5.74 \\
\hline Portugal & ECB & Loans to private non-financial enterprises with 91 to 180 days maturity & N4-2 & 2.39 \\
\hline Spain & ECB & Variable rate; monthly reviewable & N4 & 10.44 \\
\hline Australia & DS & AU major banks lending rate to small/mediu businesses (EP) & AUBSLOAN & 0.00 \\
\hline Canada & DS & CN chartered banks' prime rate (end period) & CNBANKR. & 0.00 \\
\hline Japan & DS & JP short-term prime rate (EP) & JPSPRIME & 0.00 \\
\hline Switzerland & IFS & Lending rate - Central bank: bulletin & $60 \mathrm{~b}$ & 0.00 \\
\hline United Kingdom & DS & UK major banks prime lending rate (EP) & UKBANKR. & 0.00 \\
\hline USA & DS & US prime rate charged by banks & USPRIME. & 0.00 \\
\hline \multicolumn{5}{|c|}{ Panel C: 10-year government bond yields } \\
\hline Austria & DS & OE harmonised government 10-year bond yield (Eurostat) & OEESSFUB & \\
\hline Belgium & DS & BG harmonised government 10-year bond yield (Eurostat) & BGESSFUB & \\
\hline Finland & DS & FN harmonised government 10-year bond yield (Eurostat) & FNESSFUB & \\
\hline France & DS & FR harmonised government 10-year bond yield (Eurostat) & FRESSFUB & \\
\hline Germany & DS & BD harmonised government 10-year bond yield (Eurostat) & BDESSFUB & \\
\hline Ireland & DS & IR harmonised government 10-year bond yield (Eurostat) & IRESSFUB & \\
\hline Italy & DS & IT harmonised government 10-year bond yield (Eurostat) & ITESSFUB & \\
\hline Netherlands & DS & NL harmonised government 10-year bond yield (Eurostat) & NLESSFUB & \\
\hline Portugal & DS & PT harmonised government 10-year bond yield (Eurostat) & PTESSFUB & \\
\hline Spain & DS & ES harmonised government 10-year bond yield (Eurostat) & ESESSFUB & \\
\hline Australia & DS & AU commonwealth government bond yield 10 year (EP) & AUGBOND. & \\
\hline Canada & DS & CN government bond yield: over 10 years (end month) & CNGBOND. & \\
\hline Japan & DS & JP government 10-yearYEAR bond yield (SUSP) (Eurostat) & JPESSFUB & \\
\hline Switzerland & DS & SW Switzerland 10 yr govt. bond yield (ECON, EP) & SWGBOND. & \\
\hline United Kingdom & DS & UK harmonised government 10-year bond yield (Eurostat) & UKESSFUB & \\
\hline USA & DS & US government 10-year bond yield (SUSP) (Eurostat) & USESSFUB & \\
\hline \multicolumn{5}{|c|}{ Panel D: 3-months money market rates } \\
\hline Austria & DS & OE interbank offered rate: three month (NCB) & OEINTER3 & \\
\hline Belgium & DS & BG three month interbank offered rate (EP) (NCB) & BGINTER3 & \\
\hline Finland & DS & FN HELIBOR - 3 month (mth.avg.) (NCB) & FNINTER3 & \\
\hline France & DS & FR PIBOR / EURIBOR - 3-month (mth.avg.) (OECD) & FRINTER3 & \\
\hline Germany & DS & BD FIBOR - 3 month (mth.avg.) (NCB) & BDINTER3 & \\
\hline Ireland & DS & IR three month interbank offered rate (EP) (NCB) & IRINTER3 & \\
\hline Italy & DS & IT interbank deposit rate-average on 3-months deposits (NCB) & ITINTER3 & \\
\hline Netherlands & DS & NL interbank three month: offered rate (EP) (NCB) & NLINTER3 & \\
\hline Portugal & DS & PT LISBon interbank offer rate - 3 month (EP) (NCB) & PTINTER3 & \\
\hline Spain & DS & ES interbank rate - 3 month (weighted average, EP) (Ministerio De Economia y Hacienda) & ESINTER3 & \\
\hline Australia & DS & AU interbank rate - 3 month (EP) (NCB) & AUINTER3 & \\
\hline Canada & DS & Canada treasury bill 3 mth. (BOC) - middle rate (Statistics Canada) & CNTBB3M & \\
\hline Japan & DS & JP 3 month money market rate (Eurostat) & JPESSFON & \\
\hline Switzerland & DS & SW three month interbank rate: bid rate (NCB) & SWINTER3 & \\
\hline United Kingdom & DS & UK interbank rate - 3 month (mth.avg.) (Financial Times) & UKINTER3 & \\
\hline USA & DS & US US 3 month interbank rate (London) (mth.avg.) (DS) & USINTER3 & \\
\hline
\end{tabular}

${ }^{1}$ Datasources are the European Central Bank (ECB), Datastream (DS), and the International Financial Statistics (IFS). ${ }^{2}$ In Panel C and D the original datasource is indicated in parentheses. NCB indicates the national central bank. ${ }^{3}$ Weight used in cointegration analysis for eurozone average rates. Note that weights are re-calculated to sum to 100 when a country is excluded from the average. Source: OECD Economic Outlook 73, Volume 2003/1, June, page 190, Weighting scheme for aggregate measures, based on 1995 GDP and purchasing power parities. 


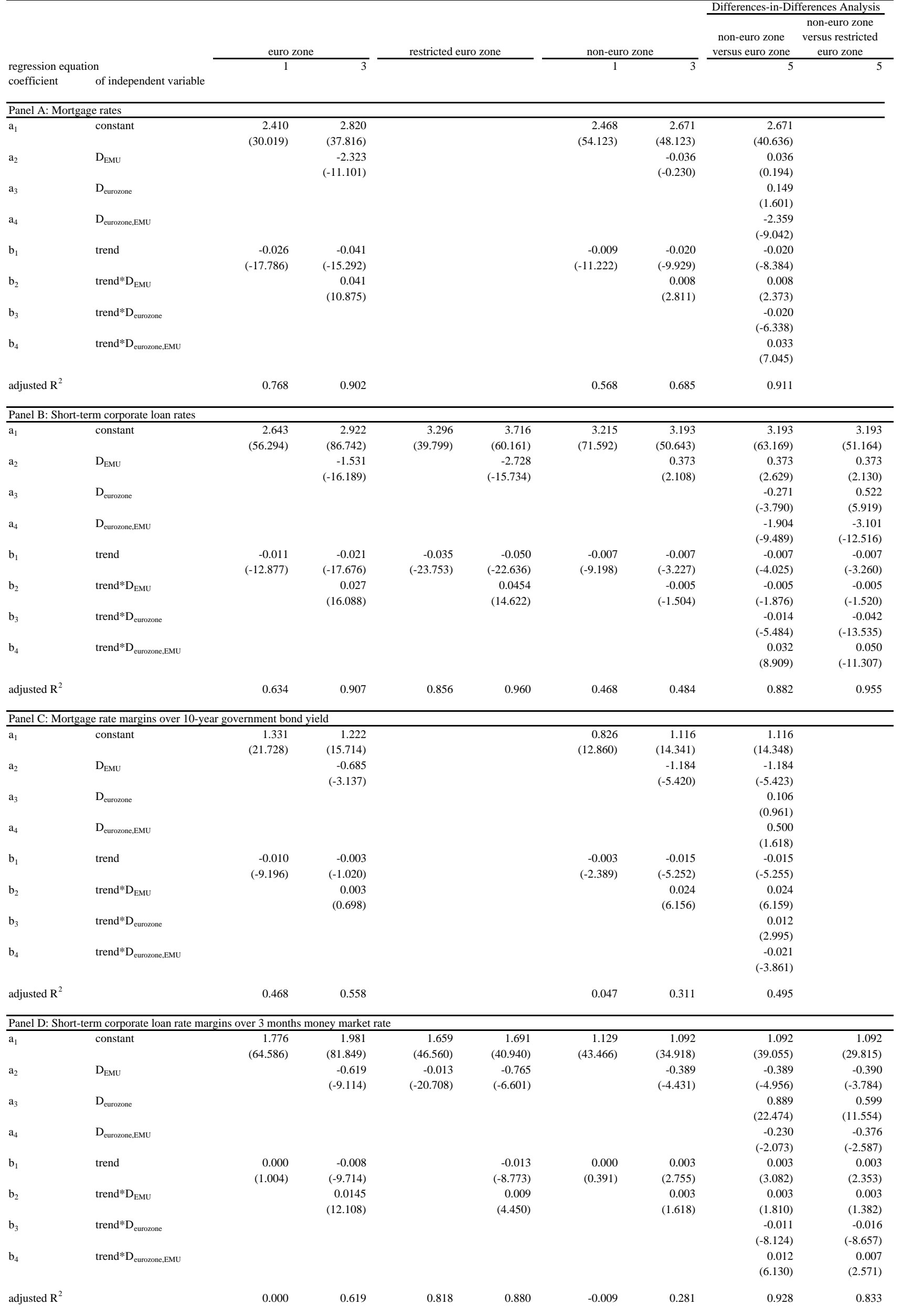

Notes: The dependent variable is the monthly standard deviation across the national rates or margins. The sample period ranges from January 1995 to December 2002 . For each regression variable, the first row reports the estimated coefficient and the second row reports the t-test statistic against zero. For euro zone and non-euro zone countries see notes to Table 1 . The restricted euro zone countries do not include Belgium, Germany, and Ireland. The number of observations are 96 for regression 1 and 3 and 192 for regression 5. 


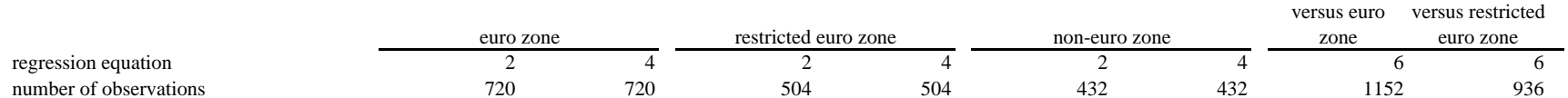

number of obser

coefficient of independent variable

\begin{tabular}{|c|c|c|c|c|c|c|c|c|c|}
\hline \multicolumn{10}{|c|}{ Panel A: Mortgage rates } \\
\hline \multirow[t]{2}{*}{$\mathrm{d}_{1}$} & $\mathrm{z}_{\mathrm{t}}$ & $\begin{array}{r}-0.270 \\
\end{array}$ & -0.257 & & & -0.144 & -0.170 & $\begin{aligned}-0.170 \\
-0.170)\end{aligned}$ & \\
\hline & & $(-17.593)$ & $(-16.454)$ & & & $(-8.492)$ & $(-7.636)$ & $(-8.195)$ & \\
\hline $\mathrm{d}_{2}$ & $\mathrm{z}_{\mathrm{t}} * \mathrm{D}_{\mathrm{EMU}}$ & & -0.248 & & & & 0.061 & 0.061 & \\
\hline$d_{3}$ & $\mathrm{z}_{\mathrm{t}} * \mathrm{D}_{\mathrm{r}=\text { eurozone }}$ & & $(-3.608)$ & & & & $(1.790)$ & $\begin{array}{r}(1.921) \\
-0.087 \\
-3\end{array}$ & \\
\hline $\mathrm{d}_{4}$ & $\mathrm{z}_{\mathrm{t}} * \mathrm{D}_{\mathrm{r}=\text { eurozone,EMU }}$ & & & & & & & $\begin{array}{r}(-3.303) \\
-0.309 \\
(-3.923)\end{array}$ & \\
\hline \multicolumn{2}{|l|}{ adjusted $\mathrm{R}^{2}$} & 0.301 & 0.312 & & & 0.143 & 0.148 & 0.249 & \\
\hline \multicolumn{10}{|c|}{ Panel B: Short-term corporate loan rates } \\
\hline \multirow[t]{2}{*}{$\overline{\mathrm{d}_{1}}$} & $\mathrm{z}_{\mathrm{t}}$ & -0.095 & -0.154 & -0.213 & -0.215 & -0.080 & -0.074 & -0.074 & -0.074 \\
\hline & & $(-8.273)$ & $(-11.423)$ & $(-17.81)$ & $(-17.189)$ & $(-5.355)$ & $(-3.632)$ & $(-4.273)$ & $(-4.407)$ \\
\hline \multirow[t]{2}{*}{$d_{2}$} & $z_{t} * D_{\text {EMU }}$ & & 0.181 & & 0.045 & & -0.013 & -0.013 & -0.013 \\
\hline & & & (7.645) & & $(0.836)$ & & $(-0.448)$ & $(-0.527)$ & $(-0.543)$ \\
\hline$d_{3}$ & $\mathrm{z}_{\mathrm{t}} * \mathrm{D}_{\mathrm{r}=\text { eurozone }}$ & & & & & & & $\begin{array}{r}-0.081 \\
(-3.495)\end{array}$ & $\begin{array}{r}-0.141 \\
(-6.073)\end{array}$ \\
\hline$d_{4}$ & $\mathrm{z}_{\mathrm{t}} * \mathrm{D}_{\mathrm{r}=\text { eurozone,EMU }}$ & & & & & & & $\begin{array}{r}0.194 \\
(5.240\end{array}$ & $\begin{array}{r}0.058 \\
(0.789)\end{array}$ \\
\hline \multicolumn{2}{|l|}{ adjusted $\mathrm{R}^{2}$} & 0.087 & 0.154 & 0.378 & 0.378 & 0.062 & 0.061 & 0.108 & 0.192 \\
\hline \multicolumn{10}{|c|}{ Panel C: Mortgage rate margins over 10-year government bond yield } \\
\hline \multirow[t]{2}{*}{$\overline{\mathrm{d}_{1}}$} & $z_{t}$ & -0.282 & -0.251 & & & -0.572 & -0.490 & -0.49 & \\
\hline & & $(-10.954)$ & $(-9.089)$ & & & $(-13.365)$ & $(-9.870)$ & $(-9.877)$ & \\
\hline \multirow[t]{2}{*}{$\mathrm{d}_{2}$} & $\mathrm{z}_{\mathrm{t}} * \mathrm{D}_{\mathrm{EMU}}$ & & -0.220 & & & & -0.305 & $\begin{array}{l}-0.305 \\
-3027\end{array}$ & \\
\hline & & & $(-2.985)$ & & & & $(-3.200)$ & $(-3.203)$ & \\
\hline \multirow[t]{2}{*}{$d_{3}$} & $\mathrm{z}_{\mathrm{t}} * \mathrm{D}_{\mathrm{r}=\text { eurozone }}$ & & & & & & & 0.239 & \\
\hline & & & & & & & & -4.203 & \\
\hline \multirow[t]{2}{*}{$\mathrm{d}_{4}$} & $\mathrm{z}_{\mathrm{t}} * \mathrm{D}_{\mathrm{r}=\text { =errozone,EMU }}$ & & & & & & & 0.085 & \\
\hline & & & & & & & & -0.0709 & \\
\hline \multicolumn{2}{|l|}{ adjusted $\mathrm{R}^{2}$} & 0.143 & 0.152 & & & 0.293 & 0.308 & 0.218 & \\
\hline \multicolumn{10}{|c|}{ Panel D: Short-term corporate loan rate margins over 3-months money market rate } \\
\hline \multirow[t]{2}{*}{$\mathrm{d}_{1}$} & $\mathrm{z}_{\mathrm{t}}$ & -0.043 & -0.109 & -0.181 & -0.182 & -0.060 & -0.025 & -0.025 & -0.025 \\
\hline & & $(-4.307)$ & $(-8.040)$ & $(-9.847)$ & $(-9.009)$ & $(-2.882)$ & $(-0.862)$ & $(-0.872)$ & $(-0.876)$ \\
\hline \multirow[t]{2}{*}{$d_{2}$} & $\mathrm{z}_{\mathrm{t}} * \mathrm{D}_{\mathrm{EMU}}$ & & 0.135 & & 0.007 & & -0.076 & -0.076 & -0.076 \\
\hline & & & $(6.961)$ & & $(0.139)$ & & $(-1.825)$ & $(-1.846)$ & $(-1.854)$ \\
\hline \multirow[t]{2}{*}{$d_{3}$} & $\mathrm{z}_{\mathrm{t}} * \mathrm{D}_{\mathrm{r}=\text { eurozone }}$ & & & & & & & -0.084 & -0.157 \\
\hline & & & & & & & & $(-2.687)$ & $(-4.524)$ \\
\hline \multirow[t]{2}{*}{$d_{4}$} & $\mathrm{z}_{\mathrm{t}} * \mathrm{D}_{\mathrm{r} \text {-eurozone,EMU }}$ & & & & & & & 0.211 & 0.083 \\
\hline & & & & & & & & -4.627 & -1.291 \\
\hline adjusted $\mathrm{R}^{2}$ & & 0.025 & 0.086 & 0.162 & 0.160 & 0.019 & 0.024 & 0.062 & 0.099 \\
\hline
\end{tabular}

Notes: The dependent variable is $\Delta \mathrm{z}_{\mathrm{t}+12}$. The sample period consists of a 3-year pre-EMU period from $\mathrm{t}=1 / 95$ to $12 / 97$ and a 3-year EMU period from $\mathrm{t}=1 / 99$ to 12/01. For each regression variable, the first row reports the estimated coefficient and the second row reports the t-test statistic against zero. For euro zone and non-euro zone countries see notes to Table 1. The restrictied euro zone countries do not include Belgium, Germany, and Ireland. 
Table A4: Differences in regional convergence based on differences-in-differences analysis for a restricted euro zone

\begin{tabular}{|c|c|c|c|c|}
\hline \multicolumn{5}{|l|}{ Panel A: Short-term corporate loan rates } \\
\hline \multirow[b]{2}{*}{ convergence coefficient for region } & \multicolumn{2}{|c|}{$\sigma$-convergence } & \multicolumn{2}{|c|}{$\beta$-convergence } \\
\hline & $\begin{array}{r}\text { pre-EMU } \\
\text { period }\end{array}$ & $\begin{array}{r}\text { EMU } \\
\text { period }\end{array}$ & $\begin{array}{r}\text { pre-EMU } \\
\text { period }\end{array}$ & $\begin{array}{l}\text { EMU } \\
\text { period }\end{array}$ \\
\hline eurozone & $-0.050 *$ & $-0.004 * *$ & $-0.215^{*}$ & $-0.170^{*}$ \\
\hline non-eurozone & $-0.007^{*}$ & $-0.012^{*}$ & $-0.074 *$ & $-0.087 *$ \\
\hline \multicolumn{5}{|l|}{ F-test for differences across coefficients } \\
\hline eurozone versus non-eurzone pre-EMU convergence & 183.18 & $0.0 \%$ & 36.887 & $0.0 \%$ \\
\hline eurozone versus non-eurzone EMU convergence & 6.029 & $1.5 \%$ & 1.413 & $23.5 \%$ \\
\hline pre-EMU versus EMU eurozone convergence & 209.388 & $0.0 \%$ & 0.416 & $51.9 \%$ \\
\hline pre-EMU versus EMU non-eurozone convergence & 2.310 & $13.0 \%$ & 0.295 & $58.7 \%$ \\
\hline
\end{tabular}

Panel B: Short-term corporate loan rate margins over 3-months money market rate

\begin{tabular}{|c|c|c|c|c|}
\hline \multirow[b]{2}{*}{ convergence coefficient for region } & \multicolumn{2}{|c|}{$\sigma$-convergence } & \multicolumn{2}{|c|}{$\beta$-convergence } \\
\hline & $\begin{array}{r}\text { pre-EMU } \\
\text { period }\end{array}$ & $\begin{array}{l}\text { EMU } \\
\text { period }\end{array}$ & $\begin{array}{r}\text { pre-EMU } \\
\text { period }\end{array}$ & $\begin{array}{l}\text { EMU } \\
\text { period }\end{array}$ \\
\hline eurozone & $-0.013^{*}$ & $-0.004^{*}$ & $-0.182^{*}$ & $-0.175^{*}$ \\
\hline non-eurozone & $0.003^{* *}$ & $0.005^{*}$ & -0.025 & $-0.101 *$ \\
\hline \multicolumn{5}{|l|}{ F-test for differences across coefficients } \\
\hline eurozone versus non-eurzone pre-EMU convergence & 74.944 & $0.0 \%$ & 20.465 & $0.0 \%$ \\
\hline eurozone versus non-eurzone EMU convergence & 25.214 & $0.0 \%$ & 1.892 & $16.9 \%$ \\
\hline pre-EMU versus EMU eurozone convergence & 25.173 & $0.0 \%$ & 0.019 & $89.1 \%$ \\
\hline pre-EMU versus EMU non-eurozone convergence & 1.909 & $16.9 \%$ & 3.438 & $6.4 \%$ \\
\hline
\end{tabular}

See notes to Table 1. The restricted euro zone excludes Belgium, Germany, and Ireland. 
Table A5: Unit root tests

\begin{tabular}{|c|c|c|c|c|c|c|c|c|c|c|c|c|c|c|c|c|c|c|}
\hline \multirow[b]{2}{*}{ national rate } & \multirow[b]{2}{*}{$\mathrm{t}($ level $)$} & \multirow[b]{2}{*}{$\mathrm{t}($ diff $)$} & \multicolumn{2}{|c|}{$\mathrm{I}(?)$} & \multirow[b]{2}{*}{ F(level) } & \multicolumn{3}{|c|}{$\mathrm{I}(?)$} & \multirow{2}{*}{\multicolumn{2}{|c|}{ corresponding eurozone average }} & \multirow[b]{2}{*}{$\mathrm{t}(\mathrm{level})$} & \multirow[b]{2}{*}{$\mathrm{t}($ diff $)$} & \multicolumn{2}{|c|}{$\mathrm{I}(?)$} & \multirow[b]{2}{*}{ F(level) } & \multirow[b]{2}{*}{$\mathrm{F}($ diff $)$} & \multicolumn{2}{|c|}{$\mathrm{I}(?)$} \\
\hline & & & $10 \%$ & $5 \%$ & & $\mathrm{~F}($ diff $)$ & $10 \%$ & $5 \%$ & & & & & $10 \%$ & $5 \%$ & & & $10 \%$ & $5 \%$ \\
\hline \multicolumn{19}{|c|}{ Panel: Mortgage rates during whole period from January 1995 to December 2002} \\
\hline Austria & -2.01 & -3.40 & 1 & 1 & 2.77 & 5.78 & 1 & 1 & eurozone average excluding & Austria & -1.05 & -4.77 & 1 & 1 & 1.01 & 11.42 & 1 & 1 \\
\hline Belgium & -2.67 & -5.20 & 0 & 1 & 4.53 & 13.53 & 1 & 1 & eurozone average excluding & Belgium & -1.39 & -4.18 & 1 & 1 & 1.93 & 8.79 & 1 & 1 \\
\hline Finland & -2.29 & -4.02 & 1 & 1 & 2.88 & 8.09 & 1 & 1 & eurozone average excluding & Finland & -1.42 & -4.12 & 1 & 1 & 2.00 & 8.53 & 1 & 1 \\
\hline France & -0.96 & -7.57 & 1 & 1 & 1.05 & 28.65 & 1 & 1 & eurozone average excluding & France & -1.56 & -4.48 & 1 & 1 & 2.14 & 10.06 & 1 & 1 \\
\hline Germany & -2.98 & -5.07 & 0 & 0 & 4.79 & 12.91 & 1 & 1 & eurozone average excluding & Germany & -1.02 & -4.08 & 1 & 1 & 1.39 & 8.53 & 1 & 1 \\
\hline Ireland & -2.41 & -6.53 & 1 & 1 & 3.12 & 21.53 & 1 & 1 & eurozone average excluding & Ireland & -1.46 & -4.07 & 1 & 1 & 2.07 & 8.31 & 1 & 1 \\
\hline Italy & -0.66 & -6.06 & 1 & 1 & 0.48 & 18.55 & 1 & 1 & eurozone average excluding & Italy & -1.95 & -4.25 & 1 & 1 & 2.70 & 9.04 & 1 & 1 \\
\hline Netherlands & -3.06 & -5.18 & 0 & 0 & 5.21 & 13.42 & 1 & 1 & eurozone average excluding & Netherlands & -1.36 & -4.09 & 1 & 1 & 1.90 & 8.41 & 1 & 1 \\
\hline Portugal & -0.94 & -3.61 & 1 & 1 & 1.03 & 6.51 & 1 & 1 & eurozone average excluding & Portugal & -1.47 & -4.16 & 1 & 1 & 2.05 & 8.67 & 1 & 1 \\
\hline Spain & -1.84 & -3.70 & 1 & 1 & 2.54 & 7.09 & 1 & 1 & eurozone average excluding & Spain & -1.46 & -4.26 & 1 & 1 & 1.99 & 9.07 & 1 & 1 \\
\hline Australia & -1.09 & -4.81 & 1 & 1 & 1.60 & 11.59 & 1 & 1 & eurozone average & & -1.45 & -4.08 & 1 & 1 & 2.03 & 8.36 & 1 & 1 \\
\hline Canada & -2.29 & -5.83 & 1 & 1 & 2.93 & 17.05 & 1 & 1 & eurozone average & & -1.45 & -4.08 & 1 & 1 & 2.03 & 8.36 & 1 & 1 \\
\hline Japan & -5.19 & -6.52 & 0 & 0 & 18.42 & 21.25 & 0 & 0 & eurozone average & & -1.45 & -4.08 & 1 & 1 & 2.03 & 8.36 & 1 & 1 \\
\hline Switzerland & -1.45 & -5.32 & 1 & 1 & 1.29 & 14.18 & 1 & 1 & eurozone average & & -1.45 & -4.08 & 1 & 1 & 2.03 & 8.36 & 1 & 1 \\
\hline United Kingdom & -2.82 & -7.30 & 0 & 1 & 3.99 & 26.64 & 1 & 1 & eurozone average & & -1.45 & -4.08 & 1 & 1 & 2.03 & 8.36 & 1 & 1 \\
\hline USA & -2.16 & -5.62 & 1 & 1 & 2.37 & 15.81 & 1 & 1 & eurozone average & & -1.45 & -4.08 & 1 & 1 & 2.03 & 8.36 & 1 & 1 \\
\hline \multicolumn{19}{|c|}{ Panel: Short-term corporate lending rates during whole period from January 1995 to December 2002} \\
\hline 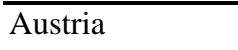 & -1.99 & -3.63 & 1 & 1 & 2.50 & 6.62 & 1 & 1 & eurozone average excluding & Austria & -0.86 & -4.01 & 1 & 1 & 0.65 & 8.04 & 1 & 1 \\
\hline Belgium & -2.06 & -6.91 & 1 & 1 & 2.76 & 24.22 & 1 & 1 & eurozone average excluding & Belgium & -1.16 & -3.64 & 1 & 1 & 0.97 & 6.68 & 1 & 1 \\
\hline Finland ifs & -2.37 & -3.19 & 1 & 1 & 3.05 & 5.11 & 2 & 2 & eurozone average excluding & Finland & -1.21 & -3.66 & 1 & 1 & 1.02 & 6.77 & 1 & 1 \\
\hline France & -1.38 & -6.73 & 1 & 1 & 0.96 & 22.66 & 1 & 1 & eurozone average excluding & France & -1.30 & -3.73 & 1 & 1 & 1.23 & 7.04 & 1 & 1 \\
\hline Germany & -1.99 & -5.51 & 1 & 1 & 4.58 & 15.17 & 1 & 1 & eurozone average excluding & Germany & -1.21 & -4.08 & 1 & 1 & 0.83 & 8.40 & 1 & 1 \\
\hline Ireland & -2.06 & -5.14 & 1 & 1 & 2.12 & 13.24 & 1 & 1 & eurozone average excluding & Ireland & -1.21 & -3.63 & 1 & 1 & 1.03 & 6.65 & 1 & 1 \\
\hline Italy & -1.64 & -3.80 & 1 & 1 & 1.42 & 7.84 & 1 & 1 & eurozone average excluding & Italy & -1.25 & -3.90 & 1 & 1 & 1.36 & 7.61 & 1 & 1 \\
\hline Netherlands & -1.91 & -4.56 & 1 & 1 & 1.91 & 10.69 & 1 & 1 & eurozone average excluding & Netherlands & -1.13 & -3.76 & 1 & 1 & 0.96 & 7.18 & 1 & 1 \\
\hline Portugal & -1.32 & -10.70 & 1 & 1 & 2.99 & 57.34 & 1 & 1 & eurozone average excluding & Portugal & -1.24 & -3.68 & 1 & 1 & 1.02 & 6.85 & 1 & 1 \\
\hline Spain & -0.83 & -6.02 & 1 & 1 & 0.79 & 18.17 & 1 & 1 & eurozone average excluding & Spain & -1.26 & -3.77 & 1 & 1 & 1.15 & 7.13 & 1 & 1 \\
\hline Australia & -1.43 & -5.11 & 1 & 1 & 1.44 & 13.03 & 1 & 1 & eurozone average & & -1.22 & -3.64 & 1 & 1 & 1.04 & 6.68 & 1 & 1 \\
\hline Canada & -2.17 & -4.70 & 1 & 1 & 2.68 & 11.13 & 1 & 1 & eurozone average & & -1.22 & -3.64 & 1 & 1 & 1.04 & 6.68 & 1 & 1 \\
\hline Japan & -6.35 & -4.65 & 0 & 0 & 24.03 & 10.81 & 0 & 0 & eurozone average & & -1.22 & -3.64 & 1 & 1 & 1.04 & 6.68 & 1 & 1 \\
\hline Switzerland & -1.50 & -5.40 & 1 & 1 & 1.23 & 14.62 & 1 & 1 & eurozone average & & -1.22 & -3.64 & 1 & 1 & 1.04 & 6.68 & 1 & 1 \\
\hline United Kingdom & -1.64 & -3.71 & 1 & 1 & 1.42 & 6.96 & 1 & 1 & eurozone average & & -1.22 & -3.64 & 1 & 1 & 1.04 & 6.68 & 1 & 1 \\
\hline USA & -0.90 & -3.80 & 1 & 1 & 0.80 & 7.21 & 1 & 1 & eurozone average & & -1.22 & -3.64 & 1 & 1 & 1.04 & 6.68 & 1 & 1 \\
\hline
\end{tabular}

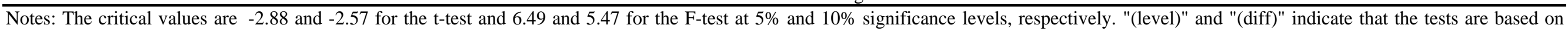
regressions in levels and first differences, respectively. 


\begin{tabular}{|c|c|c|c|c|c|c|c|c|c|}
\hline & national interest rate $r$ & sample & seriod & & coir & gration & regression & $=a+b$ & \\
\hline$\overline{r_{1}}$ & $r_{2}$ & start & end & $\mathrm{a}$ & t-stat(a) & $\mathrm{b}$ & t-stat(b) & DW & $\mathrm{DF}$ \\
\hline Panel A: & cointegration & & & & & & & & \\
\hline Austria & Belgium & 1995 & 1998 & 2.56 & 11.91 & 0.67 & 19.64 & 0.40 & -2.54 \\
\hline Austria & Belgium & 1996 & 1999 & 2.20 & 2.92 & 0.69 & 5.17 & 0.11 & -0.43 \\
\hline Austria & Belgium & 1997 & 2000 & 4.59 & 7.43 & 0.23 & 2.08 & 0.05 & -1.54 \\
\hline Austria & Belgium & 1998 & 2001 & 3.63 & 7.52 & 0.37 & 4.63 & 0.07 & -1.17 \\
\hline Austria & Belgium & 1999 & 2002 & 2.24 & 5.85 & 0.57 & 9.09 & 0.16 & -2.06 \\
\hline Austria & Finland & 1995 & 1998 & 2.82 & 19.93 & 0.60 & 28.05 & 0.33 & -2.08 \\
\hline Austria & Finland & 1996 & 1999 & 1.66 & 7.45 & 0.79 & 19.95 & 0.25 & -1.72 \\
\hline Austria & Finland & 1997 & 2000 & 1.65 & 6.03 & 0.78 & 15.54 & 0.26 & -2.65 \\
\hline Austria & Finland & 1998 & 2001 & 2.15 & 8.52 & 0.69 & 14.71 & 0.22 & -1.10 \\
\hline Austria & Finland & 1999 & 2002 & 2.67 & 12.04 & 0.59 & 13.76 & 0.18 & -1.21 \\
\hline Austria & France & 1995 & 1998 & 1.52 & 3.99 & 0.64 & 13.82 & 0.11 & -2.13 \\
\hline Austria & France & 1996 & 1999 & 2.12 & 10.27 & 0.54 & 19.32 & 0.27 & -1.83 \\
\hline Austria & France & 1997 & 2000 & 1.77 & 4.83 & 0.60 & 11.26 & 0.21 & -1.22 \\
\hline Austria & France & 1998 & 2001 & -0.45 & -1.01 & 0.96 & 14.13 & 0.61 & -3.25 \\
\hline Austria & France & 1999 & 2002 & -0.71 & -1.85 & 1.01 & 16.71 & 0.71 & -3.15 \\
\hline Austria & Germany & 1995 & 1998 & 1.77 & 6.26 & 0.79 & 17.72 & 0.30 & -2.05 \\
\hline Austria & Germany & 1996 & 1999 & 1.96 & 3.38 & 0.72 & 7.15 & 0.12 & -0.98 \\
\hline Austria & Germany & 1997 & 2000 & 3.58 & 5.98 & 0.40 & 3.85 & 0.07 & -1.35 \\
\hline Austria & Germany & 1998 & 2001 & 3.68 & 6.33 & 0.38 & 3.74 & 0.07 & -1.04 \\
\hline Austria & Germany & 1999 & 2002 & 3.12 & 5.76 & 0.45 & 4.78 & 0.10 & -1.28 \\
\hline Austria & Ireland & 1995 & 1998 & 1.66 & 1.12 & 0.71 & 3.43 & 0.07 & -2.60 \\
\hline Austria & Ireland & 1996 & 1999 & 3.14 & 8.10 & 0.45 & 7.62 & 0.10 & -2.05 \\
\hline Austria & Ireland & 1997 & 2000 & 4.11 & 15.74 & 0.29 & 6.90 & 0.10 & -0.99 \\
\hline Austria & Ireland & 1998 & 2001 & 4.26 & 13.87 & 0.28 & 5.26 & 0.07 & -0.90 \\
\hline Austria & Ireland & 1999 & 2002 & 3.06 & 7.73 & 0.52 & 6.72 & 0.14 & -1.63 \\
\hline Austria & Italy & 1995 & 1998 & 4.02 & 12.18 & 0.24 & 8.40 & 0.07 & -2.93 \\
\hline Austria & Italy & 1996 & 1999 & 4.23 & 38.14 & 0.20 & 17.39 & 0.21 & -1.76 \\
\hline Austria & Italy & 1997 & 2000 & 4.61 & 24.17 & 0.17 & 6.84 & 0.08 & 0.04 \\
\hline Austria & Italy & 1998 & 2001 & 3.70 & 11.14 & 0.32 & 6.53 & 0.09 & -1.74 \\
\hline Austria & Italy & 1999 & 2002 & 0.39 & 1.32 & 0.87 & 17.88 & 0.67 & -2.99 \\
\hline Austria & Netherlands & 1995 & 1998 & 1.21 & 3.45 & 0.86 & 15.84 & 0.29 & -1.95 \\
\hline Austria & Netherlands & 1996 & 1999 & 1.06 & 1.56 & 0.85 & 7.39 & 0.15 & -1.09 \\
\hline Austria & Netherlands & 1997 & 2000 & 2.80 & 4.33 & 0.52 & 4.78 & 0.08 & -1.64 \\
\hline Austria & Netherlands & 1998 & 2001 & 2.86 & 4.51 & 0.51 & 4.72 & 0.07 & -1.00 \\
\hline Austria & Netherlands & 1999 & 2002 & 2.33 & 4.02 & 0.58 & 5.84 & 0.10 & -1.22 \\
\hline Austria & Portugal & 1995 & 1998 & 4.10 & 16.51 & 0.26 & 10.92 & 0.06 & -1.60 \\
\hline Austria & Portugal & 1996 & 1999 & 4.30 & 35.78 & 0.22 & 15.40 & 0.14 & -1.48 \\
\hline Austria & Portugal & 1997 & 2000 & 4.53 & 25.54 & 0.20 & 7.87 & 0.09 & -0.45 \\
\hline Austria & Portugal & 1998 & 2001 & 2.79 & 11.65 & 0.52 & 12.90 & 0.17 & -2.61 \\
\hline Austria & Portugal & 1999 & 2002 & 2.17 & 11.65 & 0.64 & 19.12 & 0.28 & -1.93 \\
\hline Austria & Spain & 1995 & 1998 & 4.23 & 25.79 & 0.30 & 15.81 & 0.09 & -2.85 \\
\hline Austria & Spain & 1996 & 1999 & 4.11 & 29.14 & 0.29 & 14.43 & 0.11 & -1.08 \\
\hline Austria & Spain & 1997 & 2000 & 3.18 & 14.06 & 0.46 & 12.06 & 0.13 & -1.37 \\
\hline Austria & Spain & 1998 & 2001 & 1.44 & 9.10 & 0.79 & 27.89 & 0.53 & -2.72 \\
\hline Austria & Spain & 1999 & 2002 & 1.85 & 11.53 & 0.72 & 24.06 & 0.34 & -1.51 \\
\hline Austria & Australia & 1995 & 1998 & 3.51 & 13.83 & 0.38 & 12.98 & 0.11 & -1.96 \\
\hline Austria & Australia & 1996 & 1999 & 3.48 & 10.89 & 0.34 & 8.22 & 0.08 & -0.65 \\
\hline Austria & Australia & 1997 & 2000 & 2.35 & 3.23 & 0.50 & 4.85 & 0.08 & -0.98 \\
\hline Austria & Australia & 1998 & 2001 & 2.91 & 4.06 & 0.42 & 4.11 & 0.05 & -1.23 \\
\hline Austria & Australia & 1999 & 2002 & 2.38 & 4.37 & 0.48 & 6.13 & 0.07 & -0.76 \\
\hline Austria & Canada & 1995 & 1998 & 3.50 & 9.86 & 0.49 & 9.24 & 0.14 & -0.82 \\
\hline Austria & Canada & 1996 & 1999 & 8.63 & 10.57 & -0.41 & -3.16 & 0.07 & -2.47 \\
\hline Austria & Canada & 1997 & 2000 & 6.76 & 13.03 & -0.13 & -1.72 & 0.05 & -0.60 \\
\hline Austria & Canada & 1998 & 2001 & 5.49 & 9.84 & 0.05 & 0.65 & 0.05 & -1.01 \\
\hline Austria & Canada & 1999 & 2002 & 4.89 & 13.17 & 0.12 & 2.20 & 0.05 & -0.72 \\
\hline Austria & Japan & 1995 & 1998 & 2.82 & 6.51 & 1.40 & 9.11 & 0.16 & -1.20 \\
\hline Austria & Japan & 1996 & 1999 & -7.75 & -7.03 & 5.39 & 12.54 & 0.26 & -2.75 \\
\hline Austria & Japan & 1997 & 2000 & -1.38 & -1.21 & 2.89 & 6.38 & 0.12 & -0.94 \\
\hline Austria & Japan & 1998 & 2001 & 0.74 & 0.48 & 2.08 & 3.35 & 0.07 & -0.90 \\
\hline Austria & Japan & 1999 & 2002 & -3.08 & -1.02 & 3.66 & 2.92 & 0.10 & -1.28 \\
\hline Austria & Switzerland & 1995 & 1998 & 0.70 & 1.85 & 1.25 & 16.06 & 0.11 & -2.11 \\
\hline Austria & Switzerland & 1996 & 1999 & 0.87 & 2.30 & 1.17 & 13.86 & 0.15 & -1.34 \\
\hline Austria & Switzerland & 1997 & 2000 & 0.36 & 0.59 & 1.31 & 9.10 & 0.14 & -1.20 \\
\hline Austria & Switzerland & 1998 & 2001 & -2.02 & -2.74 & 1.89 & 10.66 & 0.23 & -1.35 \\
\hline Austria & Switzerland & 1999 & 2002 & -2.10 & -3.64 & 1.89 & 13.56 & 0.37 & -2.02 \\
\hline Austria & United Kingdom & 1995 & 1998 & 0.97 & 0.85 & 0.79 & 5.08 & 0.29 & -2.11 \\
\hline Austria & United Kingdom & 1996 & 1999 & 0.99 & 0.93 & 0.74 & 4.82 & 0.07 & -2.26 \\
\hline Austria & United Kingdom & 1997 & 2000 & 1.71 & 2.27 & 0.61 & 5.55 & 0.10 & -0.06 \\
\hline Austria & United Kingdom & 1998 & 2001 & 5.13 & 7.26 & 0.11 & 1.01 & 0.05 & -0.86 \\
\hline Austria & United Kingdom & 1999 & 2002 & 5.24 & 8.06 & 0.08 & 0.70 & 0.05 & -0.61 \\
\hline Austria & USA & 1995 & 1998 & -1.13 & -0.62 & 1.06 & 4.32 & 0.07 & -1.38 \\
\hline Austria & USA & 1996 & 1999 & -1.02 & -0.73 & 0.98 & 5.04 & 0.11 & -2.20 \\
\hline Austria & USA & 1997 & 2000 & 0.18 & 0.14 & 0.79 & 4.24 & 0.09 & -0.97 \\
\hline Austria & USA & 1998 & 2001 & 2.94 & 1.61 & 0.41 & 1.59 & 0.05 & -0.98 \\
\hline Austria & USA & 1999 & 2002 & 2.94 & 2.92 & 0.40 & 2.74 & 0.06 & -0.84 \\
\hline Belgium & Austria & 1995 & 1998 & -2.76 & -5.99 & 1.33 & 19.64 & 0.43 & -3.02 \\
\hline Belgium & Austria & 1996 & 1999 & 2.38 & 3.79 & 0.53 & 5.17 & 0.23 & -1.17 \\
\hline Belgium & Austria & 1997 & 2000 & 3.44 & 3.17 & 0.38 & 2.08 & 0.09 & -0.54 \\
\hline Belgium & Austria & 1998 & 2001 & 0.96 & 0.90 & 0.85 & 4.63 & 0.08 & -1.08 \\
\hline Belgium & Austria & 1999 & 2002 & -0.35 & -0.50 & 1.12 & 9.09 & 0.18 & -2.66 \\
\hline Belgium & Finland & 1995 & 1998 & 0.80 & 3.20 & 0.84 & 22.12 & 0.38 & -3.37 \\
\hline Belgium & Finland & 1996 & 1999 & 2.54 & 6.34 & 0.55 & 7.75 & 0.26 & -1.03 \\
\hline Belgium & Finland & 1997 & 2000 & 2.20 & 3.03 & 0.65 & 4.82 & 0.11 & -0.98 \\
\hline Belgium & Finland & 1998 & 2001 & 1.85 & 2.72 & 0.76 & 6.05 & 0.10 & -0.93 \\
\hline Belgium & Finland & 1999 & 2002 & 2.30 & 5.36 & 0.74 & 8.84 & 0.15 & -2.50 \\
\hline Belgium & France & 1995 & 1998 & -0.40 & -0.56 & 0.81 & 9.32 & 0.18 & -3.00 \\
\hline
\end{tabular}




\begin{tabular}{|c|c|c|c|c|c|c|c|c|c|}
\hline & national interest rate $\mathrm{r}$ & sample & eriod & & coin & gration & egression: & $=a+b$ & \\
\hline $\mathrm{r}_{1}$ & $\mathrm{r}_{2}$ & start & $\overline{\text { end }}$ & $\mathrm{a}$ & t-stat(a) & $\mathrm{b}$ & t-stat(b) & DW & $\mathrm{DF}$ \\
\hline Belgium & France & 1996 & 1999 & 3.15 & 7.75 & 0.34 & 6.13 & 0.30 & -1.37 \\
\hline Belgium & France & 1997 & 2000 & 5.05 & 5.48 & 0.09 & 0.69 & 0.09 & -0.29 \\
\hline Belgium & France & 1998 & 2001 & 0.56 & 0.42 & 0.82 & 4.02 & 0.12 & -1.45 \\
\hline Belgium & France & 1999 & 2002 & -1.55 & -1.74 & 1.19 & 8.56 & 0.29 & -2.68 \\
\hline Belgium & Germany & 1995 & 1998 & -1.05 & -3.90 & 1.15 & 27.24 & 0.45 & -2.00 \\
\hline Belgium & Germany & 1996 & 1999 & 1.05 & 3.56 & 0.80 & 15.46 & 0.60 & -3.05 \\
\hline Belgium & Germany & 1997 & 2000 & 0.27 & 0.67 & 0.95 & 13.50 & 0.31 & -1.55 \\
\hline Belgium & Germany & 1998 & 2001 & 0.06 & 0.12 & 1.04 & 11.92 & 0.19 & -1.58 \\
\hline Belgium & Germany & 1999 & 2002 & 0.48 & 1.12 & 0.98 & 13.14 & 0.24 & -1.71 \\
\hline Belgium & Ireland & 1995 & 1998 & -0.79 & -0.38 & 0.98 & 3.35 & 0.10 & -3.60 \\
\hline Belgium & Ireland & 1996 & 1999 & 4.97 & 9.87 & 0.10 & 1.28 & 0.15 & -1.47 \\
\hline Belgium & Ireland & 1997 & 2000 & 6.64 & 14.30 & -0.16 & -2.08 & 0.10 & -0.37 \\
\hline Belgium & Ireland & 1998 & 2001 & 6.78 & 11.87 & -0.15 & -1.52 & 0.07 & -0.93 \\
\hline Belgium & Ireland & 1999 & 2002 & 4.60 & 6.12 & 0.29 & 1.95 & 0.08 & -2.02 \\
\hline Belgium & Italy & 1995 & 1998 & 2.82 & 5.27 & 0.31 & 6.52 & 0.09 & -3.96 \\
\hline Belgium & Italy & 1996 & 1999 & 4.45 & 22.39 & 0.13 & 6.13 & 0.25 & -1.04 \\
\hline Belgium & Italy & 1997 & 2000 & 6.10 & 17.54 & -0.05 & -1.24 & 0.09 & -0.34 \\
\hline Belgium & Italy & 1998 & 2001 & 5.89 & 8.48 & 0.00 & 0.05 & 0.06 & -0.84 \\
\hline Belgium & Italy & 1999 & 2002 & 0.67 & 0.78 & 0.88 & 6.22 & 0.23 & -2.68 \\
\hline Belgium & Netherlands & 1995 & 1998 & -1.96 & -5.97 & 1.27 & 25.12 & 0.44 & -2.41 \\
\hline Belgium & Netherlands & 1996 & 1999 & 0.41 & 0.95 & 0.88 & 12.07 & 0.35 & -2.25 \\
\hline Belgium & Netherlands & 1997 & 2000 & -0.33 & -0.64 & 1.02 & 11.65 & 0.17 & -1.12 \\
\hline Belgium & Netherlands & 1998 & 2001 & -1.00 & -1.82 & 1.19 & 12.63 & 0.15 & -1.29 \\
\hline Belgium & Netherlands & 1999 & 2002 & -0.54 & -1.23 & 1.13 & 14.95 & 0.23 & -1.87 \\
\hline Belgium & Portugal & 1995 & 1998 & 2.88 & 6.72 & 0.33 & 8.04 & 0.09 & -2.63 \\
\hline Belgium & Portugal & 1996 & 1999 & 4.55 & 22.30 & 0.13 & 5.46 & 0.23 & -1.13 \\
\hline Belgium & Portugal & 1997 & 2000 & 5.88 & 16.66 & -0.03 & -0.56 & 0.09 & -0.22 \\
\hline Belgium & Portugal & 1998 & 2001 & 4.41 & 5.95 & 0.26 & 2.06 & 0.06 & -1.00 \\
\hline Belgium & Portugal & 1999 & 2002 & 2.39 & 4.27 & 0.66 & 6.59 & 0.13 & -2.64 \\
\hline Belgium & Spain & 1995 & 1998 & 3.00 & 9.39 & 0.38 & 10.48 & 0.14 & -3.62 \\
\hline Belgium & Spain & 1996 & 1999 & 4.24 & 21.03 & 0.20 & 7.07 & 0.29 & -1.21 \\
\hline Belgium & Spain & 1997 & 2000 & 5.23 & 8.75 & 0.08 & 0.77 & 0.08 & -0.30 \\
\hline Belgium & Spain & 1998 & 2001 & 1.85 & 2.28 & 0.74 & 5.06 & 0.09 & -1.20 \\
\hline Belgium & Spain & 1999 & 2002 & 1.71 & 3.26 & 0.82 & 8.31 & 0.16 & -2.47 \\
\hline Belgium & Australia & 1995 & 1998 & 2.01 & 4.55 & 0.50 & 9.75 & 0.14 & -3.00 \\
\hline Belgium & Australia & 1996 & 1999 & 3.41 & 11.65 & 0.29 & 7.66 & 0.32 & -1.52 \\
\hline Belgium & Australia & 1997 & 2000 & 0.25 & 0.30 & 0.77 & 6.43 & 0.16 & -2.35 \\
\hline Belgium & Australia & 1998 & 2001 & 0.07 & 0.08 & 0.84 & 6.39 & 0.14 & -0.85 \\
\hline Belgium & Australia & 1999 & 2002 & 1.86 & 2.27 & 0.61 & 5.13 & 0.11 & -1.67 \\
\hline Belgium & Canada & 1995 & 1998 & 1.28 & 3.12 & 0.74 & 12.25 & 0.25 & -1.33 \\
\hline Belgium & Canada & 1996 & 1999 & 4.78 & 6.12 & 0.13 & 1.07 & 0.14 & -1.66 \\
\hline Belgium & Canada & 1997 & 2000 & 2.38 & 4.81 & 0.50 & 6.74 & 0.17 & -1.51 \\
\hline Belgium & Canada & 1998 & 2001 & 3.52 & 4.60 & 0.35 & 3.17 & 0.06 & -0.41 \\
\hline Belgium & Canada & 1999 & 2002 & 4.67 & 9.21 & 0.21 & 2.76 & 0.06 & -1.81 \\
\hline Belgium & Japan & 1995 & 1998 & 0.30 & 0.58 & 2.12 & 11.51 & 0.34 & -1.92 \\
\hline Belgium & Japan & 1996 & 1999 & 0.43 & 0.23 & 2.02 & 2.75 & 0.16 & -1.28 \\
\hline Belgium & Japan & 1997 & 2000 & 10.20 & 5.29 & -1.80 & -2.34 & 0.12 & -0.46 \\
\hline Belgium & Japan & 1998 & 2001 & 11.86 & 4.91 & -2.42 & -2.46 & 0.10 & -1.15 \\
\hline Belgium & Japan & 1999 & 2002 & 5.24 & 1.14 & 0.34 & 0.18 & 0.07 & -1.55 \\
\hline Belgium & Switzerland & 1995 & 1998 & -1.40 & -1.80 & 1.58 & 9.88 & 0.16 & -2.90 \\
\hline Belgium & Switzerland & 1996 & 1999 & 2.62 & 4.30 & 0.67 & 4.94 & 0.25 & -1.20 \\
\hline Belgium & Switzerland & 1997 & 2000 & 5.53 & 4.17 & 0.04 & 0.12 & 0.08 & -0.24 \\
\hline Belgium & Switzerland & 1998 & 2001 & -1.76 & -1.01 & 1.85 & 4.43 & 0.11 & -1.20 \\
\hline Belgium & Switzerland & 1999 & 2002 & -1.46 & -1.03 & 1.82 & 5.29 & 0.14 & -2.07 \\
\hline Belgium & United Kingdom & 1995 & 1998 & -2.43 & -1.57 & 1.18 & 5.61 & 0.35 & -2.70 \\
\hline Belgium & United Kingdom & 1996 & 1999 & 2.95 & 2.76 & 0.39 & 2.50 & 0.16 & -1.87 \\
\hline Belgium & United Kingdom & 1997 & 2000 & 5.27 & 4.16 & 0.06 & 0.33 & 0.08 & -0.14 \\
\hline Belgium & United Kingdom & 1998 & 2001 & 7.23 & 6.82 & -0.20 & -1.24 & 0.08 & -0.91 \\
\hline Belgium & United Kingdom & 1999 & 2002 & 4.17 & 4.77 & 0.32 & 2.17 & 0.06 & -1.82 \\
\hline Belgium & USA & 1995 & 1998 & -5.23 & -2.06 & 1.54 & 4.52 & 0.06 & -2.23 \\
\hline Belgium & USA & 1996 & 1999 & 0.00 & 0.00 & 0.77 & 4.33 & 0.18 & -2.04 \\
\hline Belgium & USA & 1997 & 2000 & -1.07 & -0.59 & 0.94 & 3.73 & 0.10 & -0.48 \\
\hline Belgium & USA & 1998 & 2001 & -5.60 & -2.48 & 1.63 & 5.11 & 0.11 & -0.93 \\
\hline Belgium & USA & 1999 & 2002 & 1.15 & 0.86 & 0.71 & 3.66 & 0.08 & -1.91 \\
\hline Finland & Austria & 1995 & 1998 & -4.08 & -10.74 & 1.57 & 28.05 & 0.32 & -2.03 \\
\hline Finland & Austria & 1996 & 1999 & -1.30 & -3.77 & 1.13 & 19.95 & 0.25 & -1.98 \\
\hline Finland & Austria & 1997 & 2000 & -0.90 & -2.22 & 1.07 & 15.54 & 0.26 & -2.14 \\
\hline Finland & Austria & 1998 & 2001 & -1.63 & -3.43 & 1.19 & 14.71 & 0.23 & -0.95 \\
\hline Finland & Austria & 1999 & 2002 & -2.63 & -4.66 & 1.36 & 13.76 & 0.18 & -0.94 \\
\hline Finland & Belgium & 1995 & 1998 & -0.31 & -1.01 & 1.09 & 22.12 & 0.35 & -2.78 \\
\hline Finland & Belgium & 1996 & 1999 & -0.18 & -0.25 & 1.02 & 7.75 & 0.14 & -0.62 \\
\hline Finland & Belgium & 1997 & 2000 & 2.44 & 3.96 & 0.52 & 4.82 & 0.08 & -1.00 \\
\hline Finland & Belgium & 1998 & 2001 & 1.90 & 3.33 & 0.58 & 6.05 & 0.10 & -0.74 \\
\hline Finland & Belgium & 1999 & 2002 & -0.08 & -0.13 & 0.86 & 8.84 & 0.14 & -1.53 \\
\hline Finland & France & 1995 & 1998 & -1.68 & -2.46 & 1.01 & 12.07 & 0.10 & -1.71 \\
\hline Finland & France & 1996 & 1999 & 0.90 & 3.35 & 0.64 & 17.44 & 0.23 & -1.94 \\
\hline Finland & France & 1997 & 2000 & 1.32 & 2.31 & 0.60 & 7.19 & 0.14 & -0.81 \\
\hline Finland & France & 1998 & 2001 & -2.75 & -4.31 & 1.23 & 12.70 & 0.57 & -2.78 \\
\hline Finland & France & 1999 & 2002 & -4.73 & -8.99 & 1.55 & 18.72 & 0.94 & -3.52 \\
\hline Finland & Germany & 1995 & 1998 & -1.49 & -3.20 & 1.27 & 17.29 & 0.15 & -1.61 \\
\hline Finland & Germany & 1996 & 1999 & 0.07 & 0.12 & 0.96 & 9.39 & 0.12 & -1.59 \\
\hline Finland & Germany & 1997 & 2000 & 1.61 & 2.78 & 0.66 & 6.59 & 0.11 & -0.75 \\
\hline Finland & Germany & 1998 & 2001 & 1.38 & 2.14 & 0.70 & 6.18 & 0.11 & -0.84 \\
\hline Finland & Germany & 1999 & 2002 & 0.29 & 0.41 & 0.85 & 6.86 & 0.11 & -1.35 \\
\hline Finland & Ireland & 1995 & 1998 & -2.38 & -1.02 & 1.24 & 3.80 & 0.09 & -2.41 \\
\hline Finland & Ireland & 1996 & 1999 & 2.44 & 4.70 & 0.48 & 6.08 & 0.07 & -2.40 \\
\hline Finland & Ireland & 1997 & 2000 & 3.86 & 10.44 & 0.25 & 4.24 & 0.06 & 0.28 \\
\hline
\end{tabular}




\begin{tabular}{|c|c|c|c|c|c|c|c|c|c|}
\hline & national interest rate $\mathrm{r}$ & sample & eriod & & coin & gration & egression & $=a+b$ & \\
\hline$\overline{\mathrm{r}_{1}}$ & $\mathrm{r}_{2}$ & start & $\overline{\text { end }}$ & $\mathrm{a}$ & t-stat(a) & $\mathrm{b}$ & t-stat(b) & DW & $\overline{D F}$ \\
\hline Finland & Ireland & 1998 & 2001 & 3.74 & 8.31 & 0.28 & 3.60 & 0.06 & -0.61 \\
\hline Finland & Ireland & 1999 & 2002 & 1.13 & 1.88 & 0.78 & 6.65 & 0.12 & -1.40 \\
\hline Finland & Italy & 1995 & 1998 & 2.22 & 4.02 & 0.38 & 7.94 & 0.04 & -2.68 \\
\hline Finland & Italy & 1996 & 1999 & 3.38 & 24.51 & 0.24 & 16.62 & 0.16 & -1.88 \\
\hline Finland & Italy & 1997 & 2000 & 4.31 & 15.93 & 0.14 & 4.14 & 0.06 & 0.44 \\
\hline Finland & Italy & 1998 & 2001 & 3.09 & 6.13 & 0.34 & 4.52 & 0.08 & -0.89 \\
\hline Finland & Italy & 1999 & 2002 & -2.02 & -2.85 & 1.17 & 10.09 & 0.31 & -1.99 \\
\hline Finland & Netherlands & 1995 & 1998 & -2.51 & -4.69 & 1.40 & 16.94 & 0.18 & -1.66 \\
\hline Finland & Netherlands & 1996 & 1999 & -1.14 & -1.69 & 1.14 & 9.93 & 0.14 & -1.63 \\
\hline Finland & Netherlands & 1997 & 2000 & 0.60 & 1.01 & 0.81 & 8.11 & 0.11 & -1.01 \\
\hline Finland & Netherlands & 1998 & 2001 & 0.21 & 0.31 & 0.88 & 7.66 & 0.11 & -0.98 \\
\hline Finland & Netherlands & 1999 & 2002 & -0.84 & -1.12 & 1.02 & 7.95 & 0.11 & -1.09 \\
\hline Finland & Portugal & 1995 & 1998 & 2.44 & 5.47 & 0.40 & 9.36 & 0.04 & -1.28 \\
\hline Finland & Portugal & 1996 & 1999 & 3.52 & 21.23 & 0.25 & 12.89 & 0.11 & -1.71 \\
\hline Finland & Portugal & 1997 & 2000 & 4.18 & 16.22 & 0.18 & 4.90 & 0.07 & -0.10 \\
\hline Finland & Portugal & 1998 & 2001 & 1.55 & 4.18 & 0.64 & 10.33 & 0.15 & -2.06 \\
\hline Finland & Portugal & 1999 & 2002 & -0.29 & -1.10 & 0.98 & 20.92 & 0.39 & -2.32 \\
\hline Finland & Spain & 1995 & 1998 & 2.51 & 8.84 & 0.47 & 14.58 & 0.07 & -2.26 \\
\hline Finland & Spain & 1996 & 1999 & 3.18 & 20.71 & 0.35 & 16.15 & 0.13 & -1.27 \\
\hline Finland & Spain & 1997 & 2000 & 2.72 & 7.48 & 0.46 & 7.46 & 0.08 & -1.21 \\
\hline Finland & Spain & 1998 & 2001 & -0.26 & -0.88 & 1.01 & 18.97 & 0.36 & -2.13 \\
\hline Finland & Spain & 1999 & 2002 & -0.68 & -2.61 & 1.09 & 22.36 & 0.42 & -2.29 \\
\hline Finland & Australia & 1995 & 1998 & 1.38 & 3.17 & 0.60 & 11.98 & 0.09 & -1.65 \\
\hline Finland & Australia & 1996 & 1999 & 2.27 & 6.64 & 0.44 & 9.83 & 0.10 & -1.04 \\
\hline Finland & Australia & 1997 & 2000 & 0.68 & 0.87 & 0.67 & 6.04 & 0.13 & -1.52 \\
\hline Finland & Australia & 1998 & 2001 & -0.32 & -0.45 & 0.81 & 7.95 & 0.16 & -2.07 \\
\hline Finland & Australia & 1999 & 2002 & -1.17 & -1.92 & 0.91 & 10.36 & 0.21 & -0.91 \\
\hline Finland & Canada & 1995 & 1998 & 1.01 & 1.96 & 0.83 & 10.85 & 0.14 & -0.92 \\
\hline Finland & Canada & 1996 & 1999 & 7.15 & 6.80 & -0.25 & -1.51 & 0.05 & -2.58 \\
\hline Finland & Canada & 1997 & 2000 & 5.19 & 8.30 & 0.03 & 0.33 & 0.05 & -0.15 \\
\hline Finland & Canada & 1998 & 2001 & 3.00 & 4.62 & 0.34 & 3.64 & 0.07 & -1.33 \\
\hline Finland & Canada & 1999 & 2002 & 2.49 & 5.62 & 0.40 & 5.98 & 0.07 & -0.75 \\
\hline Finland & Japan & 1995 & 1998 & 0.44 & 0.59 & 2.17 & 8.18 & 0.14 & -1.15 \\
\hline Finland & Japan & 1996 & 1999 & -9.48 & -5.71 & 5.87 & 9.08 & 0.13 & -2.65 \\
\hline Finland & Japan & 1997 & 2000 & -0.41 & -0.26 & 2.32 & 3.60 & 0.06 & 0.08 \\
\hline Finland & Japan & 1998 & 2001 & 0.38 & 0.18 & 2.02 & 2.35 & 0.07 & -0.60 \\
\hline Finland & Japan & 1999 & 2002 & -9.49 & -2.12 & 6.08 & 3.27 & 0.12 & -1.13 \\
\hline Finland & Switzerland & 1995 & 1998 & -2.89 & -3.99 & 1.94 & 13.05 & 0.08 & -1.58 \\
\hline Finland & Switzerland & 1996 & 1999 & -0.42 & -0.82 & 1.35 & 11.69 & 0.13 & -1.39 \\
\hline Finland & Switzerland & 1997 & 2000 & 0.07 & 0.07 & 1.26 & 5.99 & 0.10 & -0.92 \\
\hline Finland & Switzerland & 1998 & 2001 & -3.81 & -3.17 & 2.20 & 7.62 & 0.18 & -0.35 \\
\hline Finland & Switzerland & 1999 & 2002 & -5.08 & -4.11 & 2.47 & 8.25 & 0.18 & -1.41 \\
\hline Finland & United Kingdom & 1995 & 1998 & -2.89 & -1.57 & 1.28 & 5.14 & 0.25 & -1.93 \\
\hline Finland & United Kingdom & 1996 & 1999 & 0.46 & 0.34 & 0.74 & 3.78 & 0.05 & -2.92 \\
\hline Finland & United Kingdom & 1997 & 2000 & 1.69 & 1.69 & 0.54 & 3.73 & 0.07 & 0.72 \\
\hline Finland & United Kingdom & 1998 & 2001 & 3.87 & 4.24 & 0.23 & 1.61 & 0.06 & -0.70 \\
\hline Finland & United Kingdom & 1999 & 2002 & 1.98 & 2.27 & 0.52 & 3.58 & 0.07 & -0.71 \\
\hline Finland & USA & 1995 & 1998 & -4.46 & -1.44 & 1.47 & 3.54 & 0.04 & -1.21 \\
\hline Finland & USA & 1996 & 1999 & -2.24 & -1.28 & 1.08 & 4.46 & 0.08 & -2.85 \\
\hline Finland & USA & 1997 & 2000 & -1.55 & -1.00 & 0.96 & 4.50 & 0.09 & -0.36 \\
\hline Finland & USA & 1998 & 2001 & -3.56 & -1.71 & 1.26 & 4.27 & 0.09 & -1.08 \\
\hline Finland & USA & 1999 & 2002 & -2.51 & -2.09 & 1.10 & 6.36 & 0.09 & -1.02 \\
\hline France & Austria & 1995 & 1998 & -0.33 & -0.53 & 1.26 & 13.82 & 0.12 & -1.33 \\
\hline France & Austria & 1996 & 1999 & -2.69 & -5.18 & 1.65 & 19.32 & 0.27 & -1.83 \\
\hline France & Austria & 1997 & 2000 & -0.34 & -0.54 & 1.22 & 11.26 & 0.22 & -1.67 \\
\hline France & Austria & 1998 & 2001 & 1.61 & 4.59 & 0.85 & 14.13 & 0.69 & -3.78 \\
\hline France & Austria & 1999 & 2002 & 1.50 & 5.16 & 0.85 & 16.71 & 0.76 & -3.31 \\
\hline France & Belgium & 1995 & 1998 & 3.14 & 5.78 & 0.80 & 9.32 & 0.16 & -1.61 \\
\hline France & Belgium & 1996 & 1999 & -0.18 & -0.14 & 1.33 & 6.13 & 0.18 & -0.75 \\
\hline France & Belgium & 1997 & 2000 & 6.18 & 6.76 & 0.11 & 0.69 & 0.06 & -2.04 \\
\hline France & Belgium & 1998 & 2001 & 4.67 & 9.92 & 0.32 & 4.02 & 0.19 & -2.29 \\
\hline France & Belgium & 1999 & 2002 & 3.25 & 8.89 & 0.51 & 8.56 & 0.33 & -2.35 \\
\hline France & Finland & 1995 & 1998 & 3.23 & 7.80 & 0.76 & 12.07 & 0.10 & -0.96 \\
\hline France & Finland & 1996 & 1999 & -0.27 & -0.62 & 1.36 & 17.44 & 0.23 & -1.67 \\
\hline France & Finland & 1997 & 2000 & 2.04 & 3.06 & 0.88 & 7.19 & 0.14 & -2.01 \\
\hline France & Finland & 1998 & 2001 & 3.19 & 11.95 & 0.63 & 12.70 & 0.64 & -3.41 \\
\hline France & Finland & 1999 & 2002 & 3.44 & 21.89 & 0.57 & 18.72 & 0.99 & -3.77 \\
\hline France & Germany & 1995 & 1998 & 1.72 & 3.06 & 1.02 & 11.53 & 0.18 & -2.30 \\
\hline France & Germany & 1996 & 1999 & -0.69 & -0.80 & 1.40 & 9.30 & 0.17 & -1.15 \\
\hline France & Germany & 1997 & 2000 & 3.89 & 4.43 & 0.51 & 3.35 & 0.07 & -1.96 \\
\hline France & Germany & 1998 & 2001 & 4.28 & 8.17 & 0.40 & 4.36 & 0.18 & -2.12 \\
\hline France & Germany & 1999 & 2002 & 3.75 & 7.96 & 0.46 & 5.57 & 0.19 & -1.67 \\
\hline France & Ireland & 1995 & 1998 & 3.95 & 1.76 & 0.58 & 1.87 & 0.04 & -0.94 \\
\hline France & Ireland & 1996 & 1999 & 3.27 & 3.97 & 0.62 & 4.96 & 0.06 & -1.81 \\
\hline France & Ireland & 1997 & 2000 & 4.40 & 11.40 & 0.39 & 6.37 & 0.14 & -2.10 \\
\hline France & Ireland & 1998 & 2001 & 5.18 & 17.25 & 0.24 & 4.67 & 0.22 & -1.83 \\
\hline France & Ireland & 1999 & 2002 & 4.09 & 10.60 & 0.45 & 5.95 & 0.26 & -2.07 \\
\hline France & Italy & 1995 & 1998 & 3.42 & 17.87 & 0.42 & 25.18 & 0.46 & -3.42 \\
\hline France & Italy & 1996 & 1999 & 3.89 & 49.49 & 0.37 & 45.44 & 1.14 & -4.47 \\
\hline France & Italy & 1997 & 2000 & 4.47 & 30.65 & 0.31 & 16.57 & 0.35 & -1.66 \\
\hline France & Italy & 1998 & 2001 & 4.32 & 15.64 & 0.34 & 8.17 & 0.32 & -2.08 \\
\hline France & Italy & 1999 & 2002 & 1.86 & 4.76 & 0.74 & 11.58 & 0.60 & -2.93 \\
\hline France & Netherlands & 1995 & 1998 & 1.16 & 1.66 & 1.08 & 10.07 & 0.16 & -1.73 \\
\hline France & Netherlands & 1996 & 1999 & -2.28 & -2.20 & 1.63 & 9.29 & 0.22 & -1.37 \\
\hline France & Netherlands & 1997 & 2000 & 2.78 & 2.92 & 0.68 & 4.26 & 0.10 & -2.15 \\
\hline France & Netherlands & 1998 & 2001 & 3.54 & 6.19 & 0.52 & 5.29 & 0.22 & -2.22 \\
\hline France & Netherlands & 1999 & 2002 & 3.10 & 6.07 & 0.56 & 6.41 & 0.25 & -1.81 \\
\hline
\end{tabular}




\begin{tabular}{|c|c|c|c|c|c|c|c|c|c|}
\hline & national interest rate $r$ & sample & eriod & & coin & gration & egression & $=a+b$ & \\
\hline $\mathrm{r}_{1}$ & $\mathrm{r}_{2}$ & start & $\overline{\text { end }}$ & $\mathrm{a}$ & t-stat(a) & $\mathrm{b}$ & t-stat(b) & DW & $\mathrm{DF}$ \\
\hline France & Portugal & 1995 & 1998 & 3.88 & 29.92 & 0.42 & 33.84 & 0.47 & -2.44 \\
\hline France & Portugal & 1996 & 1999 & 3.99 & 43.06 & 0.41 & 37.52 & 0.68 & -3.26 \\
\hline France & Portugal & 1997 & 2000 & 4.39 & 38.88 & 0.35 & 22.14 & 0.54 & -2.68 \\
\hline France & Portugal & 1998 & 2001 & 3.60 & 18.15 & 0.50 & 15.05 & 0.72 & -3.19 \\
\hline France & Portugal & 1999 & 2002 & 3.16 & 16.67 & 0.58 & 17.00 & 0.78 & -3.52 \\
\hline France & Spain & 1995 & 1998 & 4.39 & 39.07 & 0.44 & 34.50 & 0.47 & -2.42 \\
\hline France & Spain & 1996 & 1999 & 3.63 & 25.90 & 0.54 & 27.12 & 0.37 & -2.36 \\
\hline France & Spain & 1997 & 2000 & 2.53 & 16.81 & 0.73 & 28.74 & 0.92 & -4.10 \\
\hline France & Spain & 1998 & 2001 & 2.61 & 11.09 & 0.71 & 16.81 & 0.87 & -3.79 \\
\hline France & Spain & 1999 & 2002 & 2.87 & 17.00 & 0.66 & 20.85 & 1.01 & -3.81 \\
\hline France & Australia & 1995 & 1998 & 3.32 & 13.27 & 0.57 & 19.68 & 0.32 & -1.66 \\
\hline France & Australia & 1996 & 1999 & 2.27 & 5.07 & 0.67 & 11.43 & 0.16 & -1.17 \\
\hline France & Australia & 1997 & 2000 & 2.93 & 2.58 & 0.55 & 3.42 & 0.09 & -1.22 \\
\hline France & Australia & 1998 & 2001 & 3.90 & 5.73 & 0.38 & 3.90 & 0.20 & -2.54 \\
\hline France & Australia & 1999 & 2002 & 2.90 & 6.61 & 0.50 & 7.91 & 0.33 & -1.93 \\
\hline France & Canada & 1995 & 1998 & 5.16 & 7.24 & 0.45 & 4.25 & 0.05 & 0.15 \\
\hline France & Canada & 1996 & 1999 & 12.00 & 8.52 & -0.75 & -3.36 & 0.09 & -2.46 \\
\hline France & Canada & 1997 & 2000 & 9.61 & 15.09 & -0.42 & -4.43 & 0.11 & -1.23 \\
\hline France & Canada & 1998 & 2001 & 5.83 & 11.33 & 0.11 & 1.41 & 0.14 & -2.06 \\
\hline France & Canada & 1999 & 2002 & 5.06 & 16.75 & 0.20 & 4.36 & 0.14 & -1.31 \\
\hline France & Japan & 1995 & 1998 & 4.37 & 5.15 & 1.35 & 4.49 & 0.06 & -0.27 \\
\hline France & Japan & 1996 & 1999 & -13.78 & -5.32 & 8.22 & 8.15 & 0.12 & -2.02 \\
\hline France & Japan & 1997 & 2000 & -4.18 & -2.76 & 4.38 & 7.26 & 0.16 & -1.92 \\
\hline France & Japan & 1998 & 2001 & 1.46 & 1.04 & 2.08 & 3.61 & 0.19 & -1.70 \\
\hline France & Japan & 1999 & 2002 & -1.89 & -0.69 & 3.44 & 2.99 & 0.16 & -1.63 \\
\hline France & Switzerland & 1995 & 1998 & -0.93 & -4.50 & 1.88 & 44.06 & 0.69 & -2.99 \\
\hline France & Switzerland & 1996 & 1999 & -2.55 & -7.75 & 2.22 & 30.07 & 0.46 & -2.32 \\
\hline France & Switzerland & 1997 & 2000 & -2.27 & -4.20 & 2.15 & 16.87 & 0.47 & -2.37 \\
\hline France & Switzerland & 1998 & 2001 & -0.14 & -0.17 & 1.61 & 8.05 & 0.33 & -2.31 \\
\hline France & Switzerland & 1999 & 2002 & -0.13 & -0.19 & 1.57 & 9.36 & 0.34 & -2.03 \\
\hline France & United Kingdom & 1995 & 1998 & 3.55 & 1.89 & 0.63 & 2.46 & 0.09 & -0.76 \\
\hline France & United Kingdom & 1996 & 1999 & 0.68 & 0.34 & 0.96 & 3.25 & 0.05 & -1.92 \\
\hline France & United Kingdom & 1997 & 2000 & 0.57 & 0.55 & 0.92 & 6.08 & 0.16 & -1.53 \\
\hline France & United Kingdom & 1998 & 2001 & 5.30 & 8.22 & 0.19 & 1.95 & 0.15 & -1.76 \\
\hline France & United Kingdom & 1999 & 2002 & 4.98 & 8.80 & 0.23 & 2.45 & 0.13 & -1.27 \\
\hline France & USA & 1995 & 1998 & -5.07 & -2.19 & 1.78 & 5.71 & 0.11 & -1.06 \\
\hline France & USA & 1996 & 1999 & -7.84 & -3.75 & 2.09 & 7.25 & 0.17 & -2.77 \\
\hline France & USA & 1997 & 2000 & -4.20 & -2.69 & 1.53 & 7.05 & 0.18 & -1.81 \\
\hline France & USA & 1998 & 2001 & 2.25 & 1.37 & 0.61 & 2.63 & 0.16 & -2.01 \\
\hline France & USA & 1999 & 2002 & 2.43 & 2.99 & 0.57 & 4.84 & 0.17 & -1.42 \\
\hline Germany & Austria & 1995 & 1998 & -1.16 & -2.73 & 1.11 & 17.72 & 0.32 & -2.35 \\
\hline Germany & Austria & 1996 & 1999 & 1.28 & 2.04 & 0.73 & 7.15 & 0.17 & -1.00 \\
\hline Germany & Austria & 1997 & 2000 & 2.15 & 2.31 & 0.61 & 3.85 & 0.10 & -1.07 \\
\hline Germany & Austria & 1998 & 2001 & 2.09 & 2.19 & 0.61 & 3.74 & 0.10 & -1.07 \\
\hline Germany & Austria & 1999 & 2002 & 1.51 & 1.73 & 0.73 & 4.78 & 0.13 & -2.04 \\
\hline Germany & Belgium & 1995 & 1998 & 1.23 & 6.49 & 0.82 & 27.24 & 0.45 & -1.80 \\
\hline Germany & Belgium & 1996 & 1999 & -0.19 & -0.49 & 1.05 & 15.46 & 0.52 & -2.68 \\
\hline Germany & Belgium & 1997 & 2000 & 0.92 & 2.59 & 0.84 & 13.50 & 0.30 & -1.86 \\
\hline Germany & Belgium & 1998 & 2001 & 1.34 & 3.67 & 0.73 & 11.92 & 0.21 & -1.65 \\
\hline Germany & Belgium & 1999 & 2002 & 0.81 & 2.17 & 0.80 & 13.14 & 0.26 & -1.78 \\
\hline Germany & Finland & 1995 & 1998 & 1.86 & 7.13 & 0.68 & 17.29 & 0.18 & -2.04 \\
\hline Germany & Finland & 1996 & 1999 & 1.91 & 4.67 & 0.68 & 9.39 & 0.16 & -1.18 \\
\hline Germany & Finland & 1997 & 2000 & 1.76 & 2.93 & 0.73 & 6.59 & 0.13 & -1.24 \\
\hline Germany & Finland & 1998 & 2001 & 2.19 & 3.90 & 0.65 & 6.18 & 0.12 & -1.13 \\
\hline Germany & Finland & 1999 & 2002 & 2.63 & 5.88 & 0.60 & 6.86 & 0.14 & -2.42 \\
\hline Germany & France & 1995 & 1998 & 0.36 & 0.70 & 0.73 & 11.53 & 0.20 & -3.35 \\
\hline Germany & France & 1996 & 1999 & 2.31 & 6.23 & 0.47 & 9.30 & 0.22 & -1.17 \\
\hline Germany & France & 1997 & 2000 & 3.10 & 3.97 & 0.38 & 3.35 & 0.09 & -0.94 \\
\hline Germany & France & 1998 & 2001 & 0.88 & 0.81 & 0.73 & 4.36 & 0.13 & -1.34 \\
\hline Germany & France & 1999 & 2002 & 0.11 & 0.11 & 0.88 & 5.57 & 0.17 & -2.14 \\
\hline Germany & Ireland & 1995 & 1998 & 0.86 & 0.48 & 0.76 & 3.03 & 0.08 & -3.43 \\
\hline Germany & Ireland & 1996 & 1999 & 4.33 & 7.84 & 0.21 & 2.56 & 0.09 & -0.86 \\
\hline Germany & Ireland & 1997 & 2000 & 5.87 & 12.86 & -0.03 & -0.36 & 0.08 & -0.84 \\
\hline Germany & Ireland & 1998 & 2001 & 6.13 & 12.66 & -0.08 & -1.01 & 0.09 & -1.01 \\
\hline Germany & Ireland & 1999 & 2002 & 5.41 & 7.65 & 0.05 & 0.39 & 0.09 & -1.63 \\
\hline Germany & Italy & 1995 & 1998 & 3.09 & 7.88 & 0.29 & 8.37 & 0.10 & -5.00 \\
\hline Germany & Italy & 1996 & 1999 & 4.09 & 23.12 & 0.18 & 9.67 & 0.19 & -0.65 \\
\hline Germany & Italy & 1997 & 2000 & 5.26 & 16.15 & 0.06 & 1.42 & 0.08 & -0.70 \\
\hline Germany & Italy & 1998 & 2001 & 5.19 & 8.99 & 0.07 & 0.80 & 0.08 & -0.94 \\
\hline Germany & Italy & 1999 & 2002 & 2.71 & 2.79 & 0.49 & 3.07 & 0.12 & -2.07 \\
\hline Germany & Netherlands & 1995 & 1998 & -0.71 & -3.86 & 1.09 & 38.27 & 0.52 & -2.52 \\
\hline Germany & Netherlands & 1996 & 1999 & -0.90 & -3.34 & 1.12 & 24.77 & 0.56 & -2.74 \\
\hline Germany & Netherlands & 1997 & 2000 & -0.65 & -2.79 & 1.08 & 27.26 & 0.65 & -2.96 \\
\hline Germany & Netherlands & 1998 & 2001 & -0.80 & -4.16 & 1.11 & 33.48 & 0.89 & -3.52 \\
\hline Germany & Netherlands & 1999 & 2002 & -0.73 & -3.57 & 1.10 & 31.45 & 0.80 & -4.03 \\
\hline Germany & Portugal & 1995 & 1998 & 3.20 & 10.67 & 0.31 & 10.64 & 0.11 & -3.03 \\
\hline Germany & Portugal & 1996 & 1999 & 4.18 & 22.66 & 0.19 & 8.74 & 0.18 & -0.82 \\
\hline Germany & Portugal & 1997 & 2000 & 5.09 & 15.93 & 0.09 & 1.99 & 0.08 & -0.80 \\
\hline Germany & Portugal & 1998 & 2001 & 4.15 & 6.82 & 0.25 & 2.48 & 0.09 & -1.09 \\
\hline Germany & Portugal & 1999 & 2002 & 3.17 & 5.29 & 0.45 & 4.20 & 0.13 & -2.26 \\
\hline Germany & Spain & 1995 & 1998 & 3.53 & 13.99 & 0.33 & 11.43 & 0.14 & -3.66 \\
\hline Germany & Spain & 1996 & 1999 & 3.99 & 19.59 & 0.25 & 8.86 & 0.18 & -1.29 \\
\hline Germany & Spain & 1997 & 2000 & 4.07 & 7.96 & 0.28 & 3.24 & 0.09 & -1.03 \\
\hline Germany & Spain & 1998 & 2001 & 2.61 & 3.64 & 0.55 & 4.26 & 0.11 & -1.18 \\
\hline Germany & Spain & 1999 & 2002 & 2.67 & 4.41 & 0.57 & 5.00 & 0.14 & -2.16 \\
\hline Germany & Australia & 1995 & 1998 & 2.72 & 7.50 & 0.42 & 10.08 & 0.13 & -2.66 \\
\hline Germany & Australia & 1996 & 1999 & 3.21 & 9.43 & 0.33 & 7.48 & 0.16 & -1.40 \\
\hline
\end{tabular}




\begin{tabular}{|c|c|c|c|c|c|c|c|c|c|}
\hline & national interest rate $\mathrm{r}$ & sample & eriod & & coin & gration & regression: & $=\mathrm{a}+\mathrm{b}$ & \\
\hline $\mathrm{r}_{1}$ & $\mathrm{r}_{2}$ & start & end & $\mathrm{a}$ & t-stat(a) & b & t-stat(b) & DW & DF \\
\hline Germany & Australia & 1997 & 2000 & 0.42 & 0.54 & 0.75 & 6.87 & 0.21 & -1.94 \\
\hline Germany & Australia & 1998 & 2001 & 0.26 & 0.38 & 0.77 & 7.78 & 0.25 & -1.71 \\
\hline Germany & Australia & 1999 & 2002 & 1.55 & 2.21 & 0.60 & 5.89 & 0.19 & -1.97 \\
\hline Germany & Canada & 1995 & 1998 & 2.57 & 5.83 & 0.56 & 8.62 & 0.11 & -0.01 \\
\hline Germany & Canada & 1996 & 1999 & 6.53 & 7.24 & -0.13 & -0.90 & 0.09 & -1.22 \\
\hline Germany & Canada & 1997 & 2000 & 3.97 & 6.57 & 0.26 & 2.90 & 0.08 & -1.31 \\
\hline Germany & Canada & 1998 & 2001 & 2.54 & 4.75 & 0.46 & 5.85 & 0.10 & -0.54 \\
\hline Germany & Canada & 1999 & 2002 & 3.81 & 9.30 & 0.29 & 4.63 & 0.08 & -2.23 \\
\hline Germany & Japan & 1995 & 1998 & 1.44 & 3.09 & 1.74 & 10.57 & 0.24 & -1.08 \\
\hline Germany & Japan & 1996 & 1999 & -3.09 & -1.58 & 3.44 & 4.53 & 0.11 & -0.71 \\
\hline Germany & Japan & 1997 & 2000 & 6.36 & 3.32 & -0.26 & -0.34 & 0.08 & -0.85 \\
\hline Germany & Japan & 1998 & 2001 & 8.24 & 3.90 & -1.06 & -1.23 & 0.09 & -1.08 \\
\hline Germany & Japan & 1999 & 2002 & 7.20 & 1.73 & -0.64 & -0.37 & 0.09 & -1.42 \\
\hline Germany & Switzerland & 1995 & 1998 & -0.44 & -0.77 & 1.40 & 11.87 & 0.17 & -3.04 \\
\hline Germany & Switzerland & 1996 & 1999 & 1.47 & 2.48 & 0.96 & 7.18 & 0.18 & -0.96 \\
\hline Germany & Switzerland & 1997 & 2000 & 3.02 & 2.55 & 0.64 & 2.27 & 0.09 & -0.97 \\
\hline Germany & Switzerland & 1998 & 2001 & 1.86 & 1.13 & 0.91 & 2.31 & 0.10 & -1.03 \\
\hline Germany & Switzerland & 1999 & 2002 & 1.94 & 1.26 & 0.91 & 2.44 & 0.12 & -1.84 \\
\hline Germany & United Kingdom & 1995 & 1998 & -0.75 & -0.57 & 0.96 & 5.33 & 0.30 & -2.40 \\
\hline Germany & United Kingdom & 1996 & 1999 & 1.11 & 0.99 & 0.67 & 4.14 & 0.10 & -1.49 \\
\hline Germany & United Kingdom & 1997 & 2000 & 2.89 & 2.58 & 0.41 & 2.52 & 0.07 & -0.44 \\
\hline Germany & United Kingdom & 1998 & 2001 & 4.31 & 4.90 & 0.21 & 1.53 & 0.07 & -0.90 \\
\hline Germany & United Kingdom & 1999 & 2002 & 2.62 & 3.76 & 0.52 & 4.42 & 0.09 & -2.34 \\
\hline Germany & USA & 1995 & 1998 & -5.69 & -3.07 & 1.61 & 6.48 & 0.08 & -2.08 \\
\hline Germany & USA & 1996 & 1999 & -3.23 & -2.73 & 1.23 & 7.59 & 0.18 & -1.96 \\
\hline Germany & USA & 1997 & 2000 & -3.94 & -2.97 & 1.34 & 7.27 & 0.18 & -1.43 \\
\hline Germany & USA & 1998 & 2001 & -7.80 & -6.06 & 1.91 & 10.46 & 0.32 & -1.73 \\
\hline Germany & USA & 1999 & 2002 & -0.19 & -0.17 & 0.85 & 5.47 & 0.11 & -2.32 \\
\hline Ireland & Austria & 1995 & 1998 & 5.24 & 9.20 & 0.29 & 3.43 & 0.31 & -1.56 \\
\hline Ireland & Austria & 1996 & 1999 & -1.03 & -1.03 & 1.24 & 7.62 & 0.13 & -0.73 \\
\hline Ireland & Austria & 1997 & 2000 & -4.21 & -2.81 & 1.76 & 6.90 & 0.10 & -0.84 \\
\hline Ireland & Austria & 1998 & 2001 & -2.17 & -1.45 & 1.35 & 5.26 & 0.09 & -1.29 \\
\hline Ireland & Austria & 1999 & 2002 & -0.35 & -0.43 & 0.95 & 6.72 & 0.23 & -1.89 \\
\hline Ireland & Belgium & 1995 & 1998 & 5.93 & 15.70 & 0.20 & 3.35 & 0.32 & -1.69 \\
\hline Ireland & Belgium & 1996 & 1999 & 4.54 & 2.93 & 0.35 & 1.28 & 0.06 & 0.90 \\
\hline Ireland & Belgium & 1997 & 2000 & 9.26 & 6.08 & -0.55 & -2.08 & 0.07 & -0.96 \\
\hline Ireland & Belgium & 1998 & 2001 & 7.60 & 6.06 & -0.32 & -1.52 & 0.09 & -1.40 \\
\hline Ireland & Belgium & 1999 & 2002 & 3.46 & 4.16 & 0.27 & 1.95 & 0.15 & -1.46 \\
\hline Ireland & Finland & 1995 & 1998 & 5.92 & 17.63 & 0.19 & 3.80 & 0.33 & -1.65 \\
\hline Ireland & Finland & 1996 & 1999 & 1.34 & 1.57 & 0.93 & 6.08 & 0.10 & -0.37 \\
\hline Ireland & Finland & 1997 & 2000 & 0.09 & 0.06 & 1.12 & 4.24 & 0.06 & -0.51 \\
\hline Ireland & Finland & 1998 & 2001 & 1.51 & 1.29 & 0.79 & 3.60 & 0.07 & -1.36 \\
\hline Ireland & Finland & 1999 & 2002 & 1.88 & 3.88 & 0.63 & 6.65 & 0.20 & -1.95 \\
\hline Ireland & France & 1995 & 1998 & 6.19 & 11.64 & 0.12 & 1.87 & 0.28 & -1.22 \\
\hline Ireland & France & 1996 & 1999 & 2.40 & 2.87 & 0.56 & 4.96 & 0.10 & -0.29 \\
\hline Ireland & France & 1997 & 2000 & -1.97 & -1.55 & 1.19 & 6.37 & 0.13 & -1.39 \\
\hline Ireland & France & 1998 & 2001 & -3.01 & -1.61 & 1.33 & 4.67 & 0.15 & -1.33 \\
\hline Ireland & France & 1999 & 2002 & -1.09 & -1.05 & 0.97 & 5.95 & 0.29 & -2.13 \\
\hline Ireland & Germany & 1995 & 1998 & 5.79 & 12.56 & 0.22 & 3.03 & 0.31 & -1.73 \\
\hline Ireland & Germany & 1996 & 1999 & 3.18 & 2.43 & 0.58 & 2.56 & 0.07 & 0.70 \\
\hline Ireland & Germany & 1997 & 2000 & 6.73 & 3.97 & -0.11 & -0.36 & 0.05 & -0.92 \\
\hline Ireland & Germany & 1998 & 2001 & 7.16 & 4.96 & -0.26 & -1.01 & 0.08 & -1.39 \\
\hline Ireland & Germany & 1999 & 2002 & 4.73 & 5.28 & 0.06 & 0.39 & 0.14 & -1.14 \\
\hline Ireland & Italy & 1995 & 1998 & 6.71 & 20.45 & 0.04 & 1.45 & 0.27 & -1.12 \\
\hline Ireland & Italy & 1996 & 1999 & 4.46 & 11.25 & 0.22 & 5.41 & 0.09 & -0.38 \\
\hline Ireland & Italy & 1997 & 2000 & 2.62 & 6.50 & 0.46 & 8.93 & 0.14 & -1.64 \\
\hline Ireland & Italy & 1998 & 2001 & -0.12 & -0.22 & 0.88 & 11.21 & 0.31 & -2.33 \\
\hline Ireland & Italy & 1999 & 2002 & -0.92 & -1.29 & 0.98 & 8.47 & 0.40 & -2.49 \\
\hline Ireland & Netherlands & 1995 & 1998 & 5.37 & 10.68 & 0.28 & 3.64 & 0.32 & -1.81 \\
\hline Ireland & Netherlands & 1996 & 1999 & 1.57 & 1.04 & 0.84 & 3.30 & 0.09 & 0.69 \\
\hline Ireland & Netherlands & 1997 & 2000 & 5.48 & 2.82 & 0.11 & 0.32 & 0.05 & -0.89 \\
\hline Ireland & Netherlands & 1998 & 2001 & 6.67 & 3.94 & -0.17 & -0.57 & 0.08 & -1.37 \\
\hline Ireland & Netherlands & 1999 & 2002 & 4.19 & 4.09 & 0.15 & 0.87 & 0.14 & -1.20 \\
\hline Ireland & Portugal & 1995 & 1998 & 6.76 & 23.02 & 0.04 & 1.49 & 0.27 & -1.15 \\
\hline Ireland & Portugal & 1996 & 1999 & 4.62 & 11.53 & 0.23 & 4.95 & 0.09 & -0.25 \\
\hline Ireland & Portugal & 1997 & 2000 & 2.92 & 6.37 & 0.47 & 7.18 & 0.10 & -1.49 \\
\hline Ireland & Portugal & 1998 & 2001 & -0.32 & -0.47 & 1.02 & 8.83 & 0.16 & -1.41 \\
\hline Ireland & Portugal & 1999 & 2002 & 0.87 & 2.07 & 0.76 & 10.09 & 0.33 & -2.28 \\
\hline Ireland & Spain & 1995 & 1998 & 6.71 & 26.22 & 0.06 & 1.91 & 0.28 & -1.16 \\
\hline Ireland & Spain & 1996 & 1999 & 4.80 & 9.89 & 0.25 & 3.65 & 0.07 & 0.02 \\
\hline Ireland & Spain & 1997 & 2000 & 0.98 & 1.17 & 0.88 & 6.17 & 0.09 & -1.38 \\
\hline Ireland & Spain & 1998 & 2001 & 0.06 & 0.05 & 1.02 & 4.66 & 0.09 & -1.21 \\
\hline Ireland & Spain & 1999 & 2002 & 0.96 & 1.85 & 0.77 & 7.97 & 0.28 & -2.32 \\
\hline Ireland & Australia & 1995 & 1998 & 6.80 & 19.71 & 0.04 & 1.12 & 0.27 & -1.00 \\
\hline Ireland & Australia & 1996 & 1999 & 5.16 & 6.36 & 0.18 & 1.70 & 0.06 & 0.47 \\
\hline Ireland & Australia & 1997 & 2000 & 7.14 & 3.24 & -0.15 & -0.47 & 0.05 & -0.99 \\
\hline Ireland & Australia & 1998 & 2001 & 6.10 & 3.31 & -0.06 & -0.21 & 0.07 & -1.31 \\
\hline Ireland & Australia & 1999 & 2002 & 1.83 & 2.11 & 0.47 & 3.75 & 0.18 & -1.50 \\
\hline Ireland & Canada & 1995 & 1998 & 6.02 & 17.60 & 0.17 & 3.45 & 0.35 & -1.64 \\
\hline Ireland & Canada & 1996 & 1999 & 11.55 & 8.89 & -0.81 & -3.90 & 0.16 & -1.19 \\
\hline Ireland & Canada & 1997 & 2000 & 11.86 & 11.81 & -0.86 & -5.78 & 0.16 & -1.30 \\
\hline Ireland & Canada & 1998 & 2001 & 7.08 & 5.82 & -0.20 & -1.14 & 0.08 & -1.04 \\
\hline Ireland & Canada & 1999 & 2002 & 3.95 & 7.89 & 0.17 & 2.27 & 0.14 & -1.30 \\
\hline Ireland & Japan & 1995 & 1998 & 5.72 & 13.95 & 0.52 & 3.62 & 0.34 & -1.78 \\
\hline Ireland & Japan & 1996 & 1999 & -17.76 & -12.40 & 9.47 & 16.96 & 0.52 & -2.50 \\
\hline Ireland & Japan & 1997 & 2000 & -18.46 & -14.30 & 9.80 & 19.06 & 0.66 & -3.11 \\
\hline Ireland & Japan & 1998 & 2001 & -16.61 & -9.24 & 9.10 & 12.43 & 0.53 & -2.61 \\
\hline
\end{tabular}




\begin{tabular}{|c|c|c|c|c|c|c|c|c|c|}
\hline & national interest rate $r$ & sample & seriod & & coir & egration & regression & $=a+b$ & \\
\hline$\overline{\mathrm{r}_{1}}$ & $r_{2}$ & start & $\overline{\text { end }}$ & $\mathrm{a}$ & t-stat(a) & $\mathrm{b}$ & t-stat(b) & DW & $\mathrm{DF}$ \\
\hline Ireland & Japan & 1999 & 2002 & -12.86 & -3.62 & 7.48 & 5.05 & 0.35 & -1.94 \\
\hline Ireland & Switzerland & 1995 & 1998 & 6.03 & 10.10 & 0.24 & 1.94 & 0.28 & -1.19 \\
\hline Ireland & Switzerland & 1996 & 1999 & 1.46 & 1.20 & 1.14 & 4.18 & 0.08 & -0.31 \\
\hline Ireland & Switzerland & 1997 & 2000 & -4.58 & -2.35 & 2.54 & 5.51 & 0.10 & -1.15 \\
\hline Ireland & Switzerland & 1998 & 2001 & -2.54 & -0.92 & 1.98 & 2.98 & 0.09 & -1.10 \\
\hline Ireland & Switzerland & 1999 & 2002 & -4.22 & -3.94 & 2.25 & 8.69 & 0.40 & -2.73 \\
\hline Ireland & United Kingdom & 1995 & 1998 & 3.25 & 4.65 & 0.54 & 5.63 & 0.60 & -2.77 \\
\hline Ireland & United Kingdom & 1996 & 1999 & -3.74 & -2.44 & 1.49 & 6.69 & 0.20 & -0.38 \\
\hline Ireland & United Kingdom & 1997 & 2000 & -4.77 & -2.68 & 1.60 & 6.14 & 0.15 & -1.18 \\
\hline Ireland & United Kingdom & 1998 & 2001 & 0.56 & 0.41 & 0.80 & 3.76 & 0.09 & -1.31 \\
\hline Ireland & United Kingdom & 1999 & 2002 & 4.71 & 5.33 & 0.06 & 0.42 & 0.14 & -1.13 \\
\hline Ireland & USA & 1995 & 1998 & 6.03 & 4.40 & 0.16 & 0.84 & 0.27 & -1.00 \\
\hline Ireland & USA & 1996 & 1999 & -0.63 & -0.23 & 0.98 & 2.63 & 0.08 & 0.29 \\
\hline Ireland & USA & 1997 & 2000 & 2.50 & 0.64 & 0.50 & 0.93 & 0.05 & -0.84 \\
\hline Ireland & USA & 1998 & 2001 & 10.73 & 2.64 & -0.71 & -1.24 & 0.08 & -1.30 \\
\hline Ireland & USA & 1999 & 2002 & 1.96 & 1.41 & 0.45 & 2.23 & 0.15 & -1.43 \\
\hline Italy & Austria & 1995 & 1998 & -5.62 & -2.79 & 2.50 & 8.40 & 0.07 & -0.94 \\
\hline Italy & Austria & 1996 & 1999 & -17.22 & -11.25 & 4.36 & 17.39 & 0.19 & -1.48 \\
\hline Italy & Austria & 1997 & 2000 & -10.24 & -3.91 & 3.04 & 6.84 & 0.05 & -0.18 \\
\hline Italy & Austria & 1998 & 2001 & -2.05 & -1.54 & 1.48 & 6.53 & 0.09 & -4.11 \\
\hline Italy & Austria & 1999 & 2002 & 0.37 & 1.16 & 1.01 & 17.88 & 0.76 & -3.12 \\
\hline Italy & Belgium & 1995 & 1998 & 1.38 & 0.91 & 1.57 & 6.52 & 0.07 & -1.04 \\
\hline Italy & Belgium & 1996 & 1999 & -10.81 & -3.29 & 3.58 & 6.13 & 0.12 & 0.17 \\
\hline Italy & Belgium & 1997 & 2000 & 10.94 & 4.02 & -0.59 & -1.24 & 0.02 & -2.47 \\
\hline Italy & Belgium & 1998 & 2001 & 6.55 & 5.25 & 0.01 & 0.05 & 0.06 & -3.73 \\
\hline Italy & Belgium & 1999 & 2002 & 2.96 & 5.85 & 0.52 & 6.22 & 0.30 & -2.18 \\
\hline Italy & Finland & 1995 & 1998 & 1.38 & 1.10 & 1.51 & 7.94 & 0.05 & -0.38 \\
\hline Italy & Finland & 1996 & 1999 & -10.93 & -8.92 & 3.63 & 16.62 & 0.14 & -1.22 \\
\hline Italy & Finland & 1997 & 2000 & -2.68 & -1.07 & 1.90 & 4.14 & 0.03 & -1.07 \\
\hline Italy & Finland & 1998 & 2001 & 1.79 & 1.67 & 0.90 & 4.52 & 0.07 & -3.68 \\
\hline Italy & Finland & 1999 & 2002 & 3.09 & 10.26 & 0.59 & 10.09 & 0.39 & -2.28 \\
\hline Italy & France & 1995 & 1998 & -6.81 & -9.45 & 2.21 & 25.18 & 0.46 & -3.06 \\
\hline Italy & France & 1996 & 1999 & -10.12 & -23.48 & 2.66 & 45.44 & 1.13 & -4.32 \\
\hline Italy & France & 1997 & 2000 & -11.37 & -9.88 & 2.78 & 16.57 & 0.31 & -1.46 \\
\hline Italy & France & 1998 & 2001 & -4.87 & -3.46 & 1.75 & 8.17 & 0.24 & -2.73 \\
\hline Italy & France & 1999 & 2002 & -0.32 & -0.57 & 1.01 & 11.58 & 0.63 & -2.89 \\
\hline Italy & Germany & 1995 & 1998 & -2.06 & -1.29 & 2.10 & 8.37 & 0.08 & -2.39 \\
\hline Italy & Germany & 1996 & 1999 & -12.47 & -5.52 & 3.80 & 9.67 & 0.13 & -0.15 \\
\hline Italy & Germany & 1997 & 2000 & 3.53 & 1.22 & 0.71 & 1.42 & 0.01 & -2.31 \\
\hline Italy & Germany & 1998 & 2001 & 5.49 & 3.90 & 0.20 & 0.80 & 0.06 & -3.74 \\
\hline Italy & Germany & 1999 & 2002 & 4.12 & 6.34 & 0.35 & 3.07 & 0.17 & -1.39 \\
\hline Italy & Ireland & 1995 & 1998 & 3.64 & 0.70 & 1.05 & 1.45 & 0.02 & 0.64 \\
\hline Italy & Ireland & 1996 & 1999 & -2.17 & -1.02 & 1.76 & 5.41 & 0.04 & -1.57 \\
\hline Italy & Ireland & 1997 & 2000 & -0.85 & -0.88 & 1.38 & 8.93 & 0.10 & -2.24 \\
\hline Italy & Ireland & 1998 & 2001 & 1.87 & 4.35 & 0.83 & 11.21 & 0.29 & -3.41 \\
\hline Italy & Ireland & 1999 & 2002 & 2.95 & 7.88 & 0.62 & 8.47 & 0.40 & -2.34 \\
\hline Italy & Netherlands & 1995 & 1998 & -2.97 & -1.53 & 2.20 & 7.35 & 0.08 & -1.48 \\
\hline Italy & Netherlands & 1996 & 1999 & -16.87 & -6.28 & 4.44 & 9.76 & 0.19 & -0.55 \\
\hline Italy & Netherlands & 1997 & 2000 & 0.84 & 0.26 & 1.15 & 2.10 & 0.02 & -2.13 \\
\hline Italy & Netherlands & 1998 & 2001 & 4.63 & 2.85 & 0.34 & 1.22 & 0.06 & -3.70 \\
\hline Italy & Netherlands & 1999 & 2002 & 3.40 & 4.74 & 0.46 & 3.77 & 0.18 & -1.38 \\
\hline Italy & Portugal & 1995 & 1998 & 1.49 & 4.32 & 0.96 & 28.79 & 0.32 & -4.00 \\
\hline Italy & Portugal & 1996 & 1999 & 0.30 & 1.63 & 1.10 & 50.33 & 0.41 & -2.26 \\
\hline Italy & Portugal & 1997 & 2000 & 0.19 & 0.72 & 1.09 & 29.05 & 0.21 & -0.65 \\
\hline Italy & Portugal & 1998 & 2001 & 0.04 & 0.08 & 1.11 & 13.22 & 0.25 & -2.26 \\
\hline Italy & Portugal & 1999 & 2002 & 2.45 & 9.67 & 0.66 & 14.49 & 0.67 & -2.99 \\
\hline Italy & Spain & 1995 & 1998 & 2.98 & 6.47 & 0.97 & 18.43 & 0.11 & -0.61 \\
\hline Italy & Spain & 1996 & 1999 & -0.47 & -1.08 & 1.43 & 23.19 & 0.09 & -1.46 \\
\hline Italy & Spain & 1997 & 2000 & -4.38 & -5.24 & 2.05 & 14.49 & 0.07 & 0.81 \\
\hline Italy & Spain & 1998 & 2001 & -0.07 & -0.06 & 1.20 & 6.46 & 0.06 & -3.48 \\
\hline Italy & Spain & 1999 & 2002 & 2.13 & 8.76 & 0.75 & 16.44 & 0.63 & -3.05 \\
\hline Italy & Australia & 1995 & 1998 & 0.64 & 0.85 & 1.24 & 14.23 & 0.11 & -0.38 \\
\hline Italy & Australia & 1996 & 1999 & -3.94 & -3.09 & 1.75 & 10.52 & 0.06 & -0.15 \\
\hline Italy & Australia & 1997 & 2000 & 3.53 & 0.93 & 0.58 & 1.08 & 0.01 & -1.56 \\
\hline Italy & Australia & 1998 & 2001 & 6.36 & 3.56 & 0.03 & 0.13 & 0.06 & -3.79 \\
\hline Italy & Australia & 1999 & 2002 & 3.39 & 4.99 & 0.39 & 4.01 & 0.16 & -1.06 \\
\hline Italy & Canada & 1995 & 1998 & 6.67 & 3.70 & 0.68 & 2.55 & 0.04 & 1.01 \\
\hline Italy & Canada & 1996 & 1999 & 22.96 & 6.20 & -2.19 & -3.72 & 0.07 & -2.22 \\
\hline Italy & Canada & 1997 & 2000 & 19.55 & 13.55 & -1.79 & -8.35 & 0.14 & -1.41 \\
\hline Italy & Canada & 1998 & 2001 & 7.89 & 6.68 & -0.19 & -1.10 & 0.07 & -3.19 \\
\hline Italy & Canada & 1999 & 2002 & 5.47 & 13.36 & 0.10 & 1.57 & 0.14 & -1.01 \\
\hline Italy & Japan & 1995 & 1998 & 5.15 & 2.40 & 2.16 & 2.84 & 0.05 & 0.46 \\
\hline Italy & Japan & 1996 & 1999 & -48.78 & -7.30 & 22.65 & 8.70 & 0.11 & -1.79 \\
\hline Italy & Japan & 1997 & 2000 & -30.77 & -8.67 & 15.30 & 10.83 & 0.20 & -2.10 \\
\hline Italy & Japan & 1998 & 2001 & -12.84 & -5.73 & 7.93 & 8.69 & 0.29 & -2.93 \\
\hline Italy & Japan & 1999 & 2002 & -4.05 & -1.27 & 4.23 & 3.18 & 0.21 & -1.40 \\
\hline Italy & Switzerland & 1995 & 1998 & -8.60 & -8.20 & 4.09 & 18.98 & 0.21 & -1.77 \\
\hline Italy & Switzerland & 1996 & 1999 & -17.06 & -17.71 & 5.94 & 27.47 & 0.31 & -2.16 \\
\hline Italy & Switzerland & 1997 & 2000 & -17.84 & -8.15 & 6.03 & 11.64 & 0.10 & 0.09 \\
\hline Italy & Switzerland & 1998 & 2001 & -3.53 & -1.40 & 2.44 & 4.01 & 0.07 & -3.44 \\
\hline Italy & Switzerland & 1999 & 2002 & -2.51 & -4.60 & 2.09 & 15.79 & 0.92 & -3.80 \\
\hline Italy & United Kingdom & 1995 & 1998 & 4.26 & 0.96 & 0.95 & 1.56 & 0.03 & 0.82 \\
\hline Italy & United Kingdom & 1996 & 1999 & -9.37 & -1.73 & 2.70 & 3.45 & 0.05 & -1.36 \\
\hline Italy & United Kingdom & 1997 & 2000 & -12.50 & -4.29 & 2.95 & 6.91 & 0.13 & -1.76 \\
\hline Italy & United Kingdom & 1998 & 2001 & 1.46 & 1.10 & 0.80 & 3.91 & 0.08 & -2.79 \\
\hline Italy & United Kingdom & 1999 & 2002 & 5.87 & 8.35 & 0.04 & 0.34 & 0.14 & -0.89 \\
\hline Italy & USA & 1995 & 1998 & -20.45 & -3.99 & 4.26 & 6.18 & 0.10 & -0.86 \\
\hline
\end{tabular}




\begin{tabular}{|c|c|c|c|c|c|c|c|c|c|}
\hline & national interest rate $\mathrm{r}$ & sample & eriod & & coin & egration & egression: & $=\mathrm{a}+\mathrm{b}$ & \\
\hline$\overline{\mathrm{r}_{1}}$ & $\mathrm{r}_{2}$ & start & $\overline{\text { end }}$ & $\mathrm{a}$ & t-stat(a) & $\mathrm{b}$ & t-stat(b) & DW & $\overline{D F}$ \\
\hline Italy & USA & 1996 & 1999 & -33.53 & $\begin{array}{l}-6.38 \\
\end{array}$ & 5.90 & 8.16 & 0.15 & -2.29 \\
\hline Italy & USA & 1997 & 2000 & -18.72 & -3.37 & 3.65 & 4.74 & 0.06 & -1.22 \\
\hline Italy & USA & 1998 & 2001 & 7.10 & 1.77 & -0.07 & -0.12 & 0.06 & -3.73 \\
\hline Italy & USA & 1999 & 2002 & 3.57 & 3.23 & 0.37 & 2.29 & 0.16 & -1.26 \\
\hline Netherlands & Austria & 1995 & 1998 & -0.20 & -0.47 & 0.98 & 15.84 & 0.31 & -2.27 \\
\hline Netherlands & Austria & 1996 & 1999 & 2.02 & 3.82 & 0.64 & 7.39 & 0.21 & -1.22 \\
\hline Netherlands & Austria & 1997 & 2000 & 2.16 & 2.75 & 0.64 & 4.78 & 0.10 & -1.10 \\
\hline Netherlands & Austria & 1998 & 2001 & 2.10 & 2.65 & 0.64 & 4.72 & 0.09 & -0.93 \\
\hline Netherlands & Austria & 1999 & 2002 & 1.64 & 2.26 & 0.74 & 5.84 & 0.13 & -1.70 \\
\hline Netherlands & Belgium & 1995 & 1998 & 1.87 & 10.17 & 0.73 & 25.12 & 0.44 & -2.23 \\
\hline Netherlands & Belgium & 1996 & 1999 & 1.06 & 2.64 & 0.86 & 12.07 & 0.30 & -1.92 \\
\hline Netherlands & Belgium & 1997 & 2000 & 1.74 & 4.83 & 0.73 & 11.65 & 0.15 & -1.36 \\
\hline Netherlands & Belgium & 1998 & 2001 & 1.96 & 6.36 & 0.65 & 12.63 & 0.17 & -1.31 \\
\hline Netherlands & Belgium & 1999 & 2002 & 1.40 & 4.67 & 0.74 & 14.95 & 0.24 & -1.73 \\
\hline Netherlands & Finland & 1995 & 1998 & 2.43 & 10.14 & 0.62 & 16.94 & 0.20 & -2.09 \\
\hline Netherlands & Finland & 1996 & 1999 & 2.56 & 7.56 & 0.60 & 9.93 & 0.21 & -1.38 \\
\hline Netherlands & Finland & 1997 & 2000 & 1.99 & 4.11 & 0.72 & 8.11 & 0.13 & -1.27 \\
\hline Netherlands & Finland & 1998 & 2001 & 2.43 & 5.44 & 0.64 & 7.66 & 0.12 & -1.17 \\
\hline Netherlands & Finland & 1999 & 2002 & 2.94 & 7.97 & 0.57 & 7.95 & 0.14 & -1.91 \\
\hline Netherlands & France & 1995 & 1998 & 1.27 & 2.46 & 0.63 & 10.07 & 0.18 & -2.79 \\
\hline Netherlands & France & 1996 & 1999 & 2.96 & 9.30 & 0.40 & 9.29 & 0.29 & -1.48 \\
\hline Netherlands & France & 1997 & 2000 & 3.08 & 4.64 & 0.41 & 4.26 & 0.11 & -1.01 \\
\hline Netherlands & France & 1998 & 2001 & 1.03 & 1.14 & 0.73 & 5.29 & 0.16 & -1.43 \\
\hline Netherlands & France & 1999 & 2002 & 0.47 & 0.55 & 0.85 & 6.41 & 0.23 & -1.99 \\
\hline Netherlands & Germany & 1995 & 1998 & 0.83 & 5.61 & 0.89 & 38.27 & 0.53 & -2.53 \\
\hline Netherlands & Germany & 1996 & 1999 & 1.15 & 5.99 & 0.83 & 24.77 & 0.58 & -2.81 \\
\hline Netherlands & Germany & 1997 & 2000 & 0.92 & 4.98 & 0.87 & 27.26 & 0.64 & -2.86 \\
\hline Netherlands & Germany & 1998 & 2001 & 0.93 & 6.30 & 0.87 & 33.48 & 0.88 & -3.50 \\
\hline Netherlands & Germany & 1999 & 2002 & 0.90 & 5.67 & 0.87 & 31.45 & 0.80 & -3.85 \\
\hline Netherlands & Ireland & 1995 & 1998 & 0.74 & 0.47 & 0.79 & 3.64 & 0.10 & -3.34 \\
\hline Netherlands & Ireland & 1996 & 1999 & 4.40 & 9.68 & 0.23 & 3.30 & 0.12 & -0.93 \\
\hline Netherlands & Ireland & 1997 & 2000 & 5.77 & 14.04 & 0.02 & 0.32 & 0.07 & -0.52 \\
\hline Netherlands & Ireland & 1998 & 2001 & 6.07 & 14.06 & -0.04 & -0.57 & 0.08 & -0.89 \\
\hline Netherlands & Ireland & 1999 & 2002 & 5.31 & 8.48 & 0.11 & 0.87 & 0.08 & -1.37 \\
\hline Netherlands & Italy & 1995 & 1998 & 3.69 & 9.67 & 0.25 & 7.35 & 0.10 & -3.76 \\
\hline Netherlands & Italy & 1996 & 1999 & 4.48 & 29.72 & 0.15 & 9.76 & 0.27 & -1.06 \\
\hline Netherlands & Italy & 1997 & 2000 & 5.32 & 18.59 & 0.08 & 2.10 & 0.07 & -0.41 \\
\hline Netherlands & Italy & 1998 & 2001 & 5.22 & 10.30 & 0.09 & 1.22 & 0.07 & -0.80 \\
\hline Netherlands & Italy & 1999 & 2002 & 2.73 & 3.28 & 0.51 & 3.77 & 0.12 & -1.76 \\
\hline Netherlands & Portugal & 1995 & 1998 & 3.74 & 12.66 & 0.27 & 9.36 & 0.11 & -2.48 \\
\hline Netherlands & Portugal & 1996 & 1999 & 4.56 & 29.00 & 0.16 & 8.84 & 0.23 & -1.05 \\
\hline Netherlands & Portugal & 1997 & 2000 & 5.16 & 18.57 & 0.11 & 2.75 & 0.07 & -0.64 \\
\hline Netherlands & Portugal & 1998 & 2001 & 4.17 & 8.05 & 0.28 & 3.24 & 0.07 & -0.97 \\
\hline Netherlands & Portugal & 1999 & 2002 & 3.30 & 6.55 & 0.46 & 5.09 & 0.11 & -1.86 \\
\hline Netherlands & Spain & 1995 & 1998 & 4.03 & 15.92 & 0.28 & 9.81 & 0.13 & -2.96 \\
\hline Netherlands & Spain & 1996 & 1999 & 4.46 & 24.01 & 0.21 & 8.01 & 0.21 & -1.39 \\
\hline Netherlands & Spain & 1997 & 2000 & 4.15 & 9.48 & 0.30 & 4.05 & 0.08 & -1.11 \\
\hline Netherlands & Spain & 1998 & 2001 & 2.68 & 4.57 & 0.57 & 5.39 & 0.10 & -1.11 \\
\hline Netherlands & Spain & 1999 & 2002 & 2.85 & 5.67 & 0.57 & 6.02 & 0.13 & -1.77 \\
\hline Netherlands & Australia & 1995 & 1998 & 3.40 & 9.23 & 0.36 & 8.40 & 0.12 & -2.31 \\
\hline Netherlands & Australia & 1996 & 1999 & 3.94 & 12.19 & 0.26 & 6.11 & 0.17 & -1.39 \\
\hline Netherlands & Australia & 1997 & 2000 & 1.20 & 1.70 & 0.67 & 6.70 & 0.18 & -2.09 \\
\hline Netherlands & Australia & 1998 & 2001 & 0.80 & 1.41 & 0.72 & 8.88 & 0.24 & -1.98 \\
\hline Netherlands & Australia & 1999 & 2002 & 1.89 & 3.21 & 0.58 & 6.76 & 0.18 & -1.63 \\
\hline Netherlands & Canada & 1995 & 1998 & 3.00 & 7.78 & 0.52 & 9.05 & 0.13 & -0.49 \\
\hline Netherlands & Canada & 1996 & 1999 & 6.89 & 8.99 & -0.16 & -1.32 & 0.12 & -1.40 \\
\hline Netherlands & Canada & 1997 & 2000 & 4.69 & 8.32 & 0.18 & 2.18 & 0.07 & -0.87 \\
\hline Netherlands & Canada & 1998 & 2001 & 3.12 & 6.52 & 0.40 & 5.69 & 0.11 & -1.14 \\
\hline Netherlands & Canada & 1999 & 2002 & 4.20 & 11.44 & 0.25 & 4.57 & 0.09 & -1.68 \\
\hline Netherlands & Japan & 1995 & 1998 & 1.89 & 4.94 & 1.63 & 12.02 & 0.29 & -1.49 \\
\hline Netherlands & Japan & 1996 & 1999 & -2.62 & -1.67 & 3.32 & 5.42 & 0.16 & -0.84 \\
\hline Netherlands & Japan & 1997 & 2000 & 5.23 & 3.04 & 0.27 & 0.39 & 0.07 & -0.51 \\
\hline Netherlands & Japan & 1998 & 2001 & 7.20 & 3.80 & -0.56 & -0.72 & 0.08 & -0.93 \\
\hline Netherlands & Japan & 1999 & 2002 & 4.68 & 1.26 & 0.49 & 0.32 & 0.08 & -1.28 \\
\hline Netherlands & Switzerland & 1995 & 1998 & 0.55 & 0.97 & 1.22 & 10.44 & 0.15 & -2.59 \\
\hline Netherlands & Switzerland & 1996 & 1999 & 2.33 & 4.44 & 0.80 & 6.83 & 0.21 & -1.16 \\
\hline Netherlands & Switzerland & 1997 & 2000 & 2.79 & 2.72 & 0.74 & 3.03 & 0.09 & -0.95 \\
\hline Netherlands & Switzerland & 1998 & 2001 & 1.74 & 1.23 & 0.98 & 2.90 & 0.10 & -0.89 \\
\hline Netherlands & Switzerland & 1999 & 2002 & 2.02 & 1.51 & 0.93 & 2.85 & 0.12 & -1.55 \\
\hline Netherlands & United Kingdom & 1995 & 1998 & -0.31 & -0.27 & 0.92 & 5.87 & 0.35 & -2.52 \\
\hline Netherlands & United Kingdom & 1996 & 1999 & 1.52 & 1.66 & 0.63 & 4.76 & 0.15 & -1.72 \\
\hline Netherlands & United Kingdom & 1997 & 2000 & 3.14 & 3.16 & 0.41 & 2.78 & 0.07 & -0.02 \\
\hline Netherlands & United Kingdom & 1998 & 2001 & 4.65 & 5.97 & 0.18 & 1.52 & 0.06 & -0.84 \\
\hline Netherlands & United Kingdom & 1999 & 2002 & 3.33 & 5.20 & 0.42 & 3.97 & 0.08 & -1.68 \\
\hline Netherlands & USA & 1995 & 1998 & -4.31 & -2.56 & 1.45 & 6.38 & 0.07 & -1.95 \\
\hline Netherlands & USA & 1996 & 1999 & -1.88 & -1.89 & 1.07 & 7.80 & 0.21 & -2.07 \\
\hline Netherlands & USA & 1997 & 2000 & -3.18 & -2.81 & 1.26 & 8.04 & 0.19 & -1.36 \\
\hline Netherlands & USA & 1998 & 2001 & -5.80 & -4.83 & 1.65 & 9.69 & 0.29 & -1.89 \\
\hline Netherlands & USA & 1999 & 2002 & 0.99 & 0.99 & 0.70 & 4.87 & 0.11 & -1.70 \\
\hline Portugal & Austria & 1995 & 1998 & -8.48 & -4.96 & 2.75 & 10.92 & 0.05 & -0.25 \\
\hline Portugal & Austria & 1996 & 1999 & -15.33 & -10.00 & 3.87 & 15.40 & 0.12 & -1.16 \\
\hline Portugal & Austria & 1997 & 2000 & -10.23 & -4.71 & 2.90 & 7.87 & 0.05 & -1.21 \\
\hline Portugal & Austria & 1998 & 2001 & -2.95 & -4.27 & 1.52 & 12.90 & 0.17 & -3.55 \\
\hline Portugal & Austria & 1999 & 2002 & -2.40 & -5.76 & 1.39 & 19.12 & 0.28 & -1.83 \\
\hline Portugal & Belgium & 1995 & 1998 & -0.86 & -0.63 & 1.75 & 8.04 & 0.06 & -0.57 \\
\hline Portugal & Belgium & 1996 & 1999 & -8.79 & -2.82 & 3.02 & 5.46 & 0.10 & 0.09 \\
\hline Portugal & Belgium & 1997 & 2000 & 8.20 & 3.32 & -0.24 & -0.56 & 0.01 & -3.61 \\
\hline
\end{tabular}




\begin{tabular}{|c|c|c|c|c|c|c|c|c|c|}
\hline & national interest rate $\mathrm{r}$ & sample & eriod & & coin & egration & regression: & $=\mathrm{a}+\mathrm{b}$ & \\
\hline $\mathrm{r}_{1}$ & $\mathrm{r}_{2}$ & start & end & $\mathrm{a}$ & t-stat(a) & b & t-stat(b) & DW & DF \\
\hline Portugal & Belgium & 1998 & 2001 & 3.96 & 4.14 & 0.33 & 2.06 & 0.05 & -2.46 \\
\hline Portugal & Belgium & 1999 & 2002 & 1.09 & 1.61 & 0.73 & 6.59 & 0.12 & -1.77 \\
\hline Portugal & Finland & 1995 & 1998 & -0.49 & -0.43 & 1.62 & 9.36 & 0.04 & 0.15 \\
\hline Portugal & Finland & 1996 & 1999 & -9.27 & -6.80 & 3.13 & 12.89 & 0.09 & -0.96 \\
\hline Portugal & Finland & 1997 & 2000 & -3.53 & -1.66 & 1.92 & 4.90 & 0.03 & -2.44 \\
\hline Portugal & Finland & 1998 & 2001 & 0.10 & 0.17 & 1.09 & 10.33 & 0.14 & -3.29 \\
\hline Portugal & Finland & 1999 & 2002 & 0.79 & 3.47 & 0.93 & 20.92 & 0.39 & -2.40 \\
\hline Portugal & France & 1995 & 1998 & -8.40 & -15.27 & 2.27 & 33.84 & 0.46 & -2.37 \\
\hline Portugal & France & 1996 & 1999 & -9.25 & -19.73 & 2.39 & 37.52 & 0.66 & -3.09 \\
\hline Portugal & France & 1997 & 2000 & -10.74 & -13.48 & 2.58 & 22.14 & 0.50 & -2.69 \\
\hline Portugal & France & 1998 & 2001 & -5.00 & -6.87 & 1.67 & 15.05 & 0.64 & -3.18 \\
\hline Portugal & France & 1999 & 2002 & -3.95 & -7.07 & 1.49 & 17.00 & 0.72 & -3.31 \\
\hline Portugal & Germany & 1995 & 1998 & -4.46 & -3.23 & 2.30 & 10.64 & 0.08 & -1.59 \\
\hline Portugal & Germany & 1996 & 1999 & -10.78 & -4.95 & 3.31 & 8.74 & 0.12 & -0.37 \\
\hline Portugal & Germany & 1997 & 2000 & 1.81 & 0.71 & 0.88 & 1.99 & 0.02 & -3.09 \\
\hline Portugal & Germany & 1998 & 2001 & 3.29 & 3.07 & 0.47 & 2.48 & 0.06 & -2.30 \\
\hline Portugal & Germany & 1999 & 2002 & 2.05 & 2.47 & 0.61 & 4.20 & 0.10 & -1.31 \\
\hline Portugal & Ireland & 1995 & 1998 & 2.27 & 0.43 & 1.09 & 1.49 & 0.02 & -0.51 \\
\hline Portugal & Ireland & 1996 & 1999 & -1.59 & -0.80 & 1.50 & 4.95 & 0.04 & -1.54 \\
\hline Portugal & Ireland & 1997 & 2000 & -0.09 & -0.09 & 1.13 & 7.18 & 0.06 & -2.78 \\
\hline Portugal & Ireland & 1998 & 2001 & 2.40 & 5.92 & 0.62 & 8.83 & 0.15 & -1.92 \\
\hline Portugal & Ireland & 1999 & 2002 & 0.93 & 2.03 & 0.91 & 10.09 & 0.25 & -1.93 \\
\hline Portugal & Italy & 1995 & 1998 & -0.94 & -2.41 & 0.98 & 28.79 & 0.31 & -4.47 \\
\hline Portugal & Italy & 1996 & 1999 & -0.13 & -0.74 & 0.90 & 50.33 & 0.41 & -2.26 \\
\hline Portugal & Italy & 1997 & 2000 & 0.19 & 0.79 & 0.87 & 29.05 & 0.21 & -0.88 \\
\hline Portugal & Italy & 1998 & 2001 & 1.21 & 3.34 & 0.71 & 13.22 & 0.24 & -1.64 \\
\hline Portugal & Italy & 1999 & 2002 & -2.05 & -3.92 & 1.24 & 14.49 & 0.59 & -2.81 \\
\hline Portugal & Netherlands & 1995 & 1998 & -5.70 & -3.36 & 2.45 & 9.36 & 0.08 & -0.99 \\
\hline Portugal & Netherlands & 1996 & 1999 & -14.65 & -5.66 & 3.87 & 8.84 & 0.15 & -0.52 \\
\hline Portugal & Netherlands & 1997 & 2000 & -0.87 & -0.31 & 1.30 & 2.75 & 0.02 & -3.27 \\
\hline Portugal & Netherlands & 1998 & 2001 & 2.07 & 1.73 & 0.66 & 3.24 & 0.06 & -2.39 \\
\hline Portugal & Netherlands & 1999 & 2002 & 0.95 & 1.06 & 0.78 & 5.09 & 0.08 & -1.16 \\
\hline Portugal & Spain & 1995 & 1998 & 1.67 & 3.97 & 1.00 & 20.68 & 0.06 & -0.42 \\
\hline Portugal & Spain & 1996 & 1999 & -0.57 & -1.33 & 1.28 & 21.34 & 0.05 & -1.39 \\
\hline Portugal & Spain & 1997 & 2000 & -4.60 & -9.62 & 1.95 & 24.16 & 0.12 & -0.55 \\
\hline Portugal & Spain & 1998 & 2001 & -1.15 & -2.43 & 1.28 & 15.00 & 0.13 & -2.94 \\
\hline Portugal & Spain & 1999 & 2002 & -0.24 & -1.37 & 1.09 & 32.60 & 0.64 & -3.34 \\
\hline Portugal & Australia & 1995 & 1998 & -0.65 & -0.88 & 1.26 & 14.72 & 0.09 & -0.27 \\
\hline Portugal & Australia & 1996 & 1999 & -3.62 & -3.06 & 1.56 & 10.13 & 0.06 & -0.32 \\
\hline Portugal & Australia & 1997 & 2000 & -0.88 & -0.27 & 1.09 & 2.39 & 0.02 & -1.56 \\
\hline Portugal & Australia & 1998 & 2001 & 2.05 & 1.55 & 0.56 & 2.95 & 0.05 & -3.44 \\
\hline Portugal & Australia & 1999 & 2002 & -0.05 & -0.07 & 0.81 & 8.01 & 0.12 & -0.92 \\
\hline Portugal & Canada & 1995 & 1998 & 4.40 & 2.51 & 0.85 & 3.29 & 0.03 & 1.11 \\
\hline Portugal & Canada & 1996 & 1999 & 22.17 & 6.93 & -2.24 & -4.40 & 0.09 & -2.43 \\
\hline Portugal & Canada & 1997 & 2000 & 16.64 & 11.57 & -1.47 & -6.89 & 0.09 & -1.15 \\
\hline Portugal & Canada & 1998 & 2001 & 5.25 & 5.50 & 0.10 & 0.71 & 0.05 & -2.55 \\
\hline Portugal & Canada & 1999 & 2002 & 3.61 & 7.23 & 0.29 & 3.88 & 0.08 & -0.83 \\
\hline Portugal & Japan & 1995 & 1998 & 1.78 & 0.89 & 2.97 & 4.18 & 0.04 & 0.32 \\
\hline Portugal & Japan & 1996 & 1999 & -42.44 & -6.67 & 19.75 & 7.96 & 0.10 & -1.68 \\
\hline Portugal & Japan & 1997 & 2000 & -24.46 & -6.42 & 12.47 & 8.22 & 0.13 & -2.26 \\
\hline Portugal & Japan & 1998 & 2001 & -7.43 & -3.44 & 5.45 & 6.19 & 0.17 & -1.80 \\
\hline Portugal & Japan & 1999 & 2002 & -11.82 & -2.88 & 7.23 & 4.23 & 0.19 & -1.55 \\
\hline Portugal & Switzerland & 1995 & 1998 & -10.69 & -15.97 & 4.29 & 31.20 & 0.18 & -0.76 \\
\hline Portugal & Switzerland & 1996 & 1999 & -15.88 & -22.85 & 5.42 & 34.78 & 0.27 & -2.21 \\
\hline Portugal & Switzerland & 1997 & 2000 & -17.93 & -13.32 & 5.87 & 18.42 & 0.23 & -1.25 \\
\hline Portugal & Switzerland & 1998 & 2001 & -5.65 & -3.47 & 2.79 & 7.11 & 0.13 & -1.88 \\
\hline Portugal & Switzerland & 1999 & 2002 & -5.65 & -6.02 & 2.71 & 11.92 & 0.32 & -2.07 \\
\hline Portugal & United Kingdom & 1995 & 1998 & 0.05 & 0.01 & 1.37 & 2.30 & 0.06 & -0.44 \\
\hline Portugal & United Kingdom & 1996 & 1999 & -7.91 & -1.60 & 2.33 & 3.26 & 0.04 & -1.39 \\
\hline Portugal & United Kingdom & 1997 & 2000 & -8.99 & -3.09 & 2.32 & 5.46 & 0.09 & -1.72 \\
\hline Portugal & United Kingdom & 1998 & 2001 & 2.88 & 2.52 & 0.47 & 2.69 & 0.07 & -1.97 \\
\hline Portugal & United Kingdom & 1999 & 2002 & 3.83 & 4.11 & 0.29 & 1.83 & 0.06 & -0.62 \\
\hline Portugal & USA & 1995 & 1998 & -25.11 & -5.34 & 4.73 & 7.49 & 0.11 & -1.00 \\
\hline Portugal & USA & 1996 & 1999 & -32.13 & -7.24 & 5.55 & 9.09 & 0.15 & -2.55 \\
\hline Portugal & USA & 1997 & 2000 & -20.93 & -4.66 & 3.84 & 6.19 & 0.07 & -1.75 \\
\hline Portugal & USA & 1998 & 2001 & 1.51 & 0.48 & 0.63 & 1.41 & 0.06 & -2.39 \\
\hline Portugal & USA & 1999 & 2002 & -0.05 & -0.04 & 0.81 & 4.06 & 0.08 & -0.94 \\
\hline Spain & Austria & 1995 & 1998 & -10.71 & -8.80 & 2.84 & 15.81 & 0.08 & -1.94 \\
\hline Spain & Austria & 1996 & 1999 & -10.46 & -8.70 & 2.85 & 14.43 & 0.09 & -1.42 \\
\hline Spain & Austria & 1997 & 2000 & -3.83 & -4.76 & 1.65 & 12.06 & 0.10 & -2.39 \\
\hline Spain & Austria & 1998 & 2001 & -1.41 & -5.63 & 1.19 & 27.89 & 0.53 & -2.79 \\
\hline Spain & Austria & 1999 & 2002 & -1.98 & -6.52 & 1.28 & 24.06 & 0.34 & -1.30 \\
\hline Spain & Belgium & 1995 & 1998 & -3.02 & -2.73 & 1.84 & 10.48 & 0.11 & -2.04 \\
\hline Spain & Belgium & 1996 & 1999 & -7.69 & -3.73 & 2.58 & 7.07 & 0.15 & -0.76 \\
\hline Spain & Belgium & 1997 & 2000 & 4.91 & 4.05 & 0.16 & 0.77 & 0.02 & -4.08 \\
\hline Spain & Belgium & 1998 & 2001 & 2.66 & 4.66 & 0.48 & 5.06 & 0.08 & -1.40 \\
\hline Spain & Belgium & 1999 & 2002 & 0.87 & 1.61 & 0.74 & 8.31 & 0.14 & -1.52 \\
\hline Spain & Finland & 1995 & 1998 & -2.85 & -3.62 & 1.74 & 14.58 & 0.06 & -1.35 \\
\hline Spain & Finland & 1996 & 1999 & -6.69 & -7.93 & 2.43 & 16.15 & 0.11 & -1.20 \\
\hline Spain & Finland & 1997 & 2000 & -0.60 & -0.69 & 1.19 & 7.46 & 0.05 & -4.01 \\
\hline Spain & Finland & 1998 & 2001 & 0.86 & 3.45 & 0.88 & 18.97 & 0.35 & -2.32 \\
\hline Spain & Finland & 1999 & 2002 & 1.02 & 5.26 & 0.84 & 22.36 & 0.41 & -2.28 \\
\hline Spain & France & 1995 & 1998 & -9.20 & -17.86 & 2.17 & 34.50 & 0.46 & -2.40 \\
\hline Spain & France & 1996 & 1999 & -5.95 & -12.52 & 1.75 & 27.12 & 0.35 & -2.56 \\
\hline Spain & France & 1997 & 2000 & -2.97 & -9.63 & 1.29 & 28.74 & 0.89 & -4.29 \\
\hline Spain & France & 1998 & 2001 & -2.38 & -5.05 & 1.21 & 16.81 & 0.79 & -3.36 \\
\hline Spain & France & 1999 & 2002 & -3.44 & -8.17 & 1.38 & 20.85 & 0.95 & -3.54 \\
\hline
\end{tabular}




\begin{tabular}{|c|c|c|c|c|c|c|c|c|c|}
\hline & national interest rate $\mathrm{r}$ & sample & eriod & & coir & egration I & egression & $=\mathrm{a}+\mathrm{b}$ & \\
\hline $\mathrm{r}_{1}$ & $\mathrm{r}_{2}$ & start & end & $\mathrm{a}$ & t-stat(a) & $\mathrm{b}$ & t-stat(b) & DW & DF \\
\hline Spain & Germany & 1995 & 1998 & -5.70 & -4.57 & 2.24 & 11.43 & 0.11 & -2.45 \\
\hline Spain & Germany & 1996 & 1999 & -7.34 & -4.57 & 2.47 & 8.86 & 0.12 & -1.61 \\
\hline Spain & Germany & 1997 & 2000 & 2.06 & 1.75 & 0.66 & 3.24 & 0.03 & -3.41 \\
\hline Spain & Germany & 1998 & 2001 & 2.62 & 3.82 & 0.52 & 4.26 & 0.08 & -1.26 \\
\hline Spain & Germany & 1999 & 2002 & 1.78 & 2.51 & 0.62 & 5.00 & 0.09 & -1.03 \\
\hline Spain & Ireland & 1995 & 1998 & -1.00 & -0.20 & 1.31 & 1.91 & 0.02 & -0.87 \\
\hline Spain & Ireland & 1996 & 1999 & 0.97 & 0.60 & 0.90 & 3.65 & 0.03 & -2.85 \\
\hline Spain & Ireland & 1997 & 2000 & 2.69 & 5.17 & 0.52 & 6.17 & 0.07 & -3.00 \\
\hline Spain & Ireland & 1998 & 2001 & 3.74 & 9.57 & 0.31 & 4.66 & 0.07 & -0.92 \\
\hline Spain & Ireland & 1999 & 2002 & 1.51 & 3.15 & 0.75 & 7.97 & 0.19 & -1.84 \\
\hline Spain & Italy & 1995 & 1998 & -1.69 & -3.02 & 0.91 & 18.43 & 0.10 & -1.31 \\
\hline Spain & Italy & 1996 & 1999 & 0.84 & 3.12 & 0.64 & 23.19 & 0.09 & -2.25 \\
\hline Spain & Italy & 1997 & 2000 & 2.80 & 12.91 & 0.40 & 14.49 & 0.07 & -0.02 \\
\hline Spain & Italy & 1998 & 2001 & 2.93 & 7.18 & 0.39 & 6.46 & 0.06 & -0.99 \\
\hline Spain & Italy & 1999 & 2002 & -1.66 & -3.91 & 1.14 & 16.44 & 0.54 & -2.80 \\
\hline Spain & Netherlands & 1995 & 1998 & -6.85 & -4.37 & 2.37 & 9.81 & 0.10 & -1.76 \\
\hline Spain & Netherlands & 1996 & 1999 & -9.50 & -4.65 & 2.77 & 8.01 & 0.13 & -1.59 \\
\hline Spain & Netherlands & 1997 & 2000 & 0.67 & 0.52 & 0.88 & 4.05 & 0.04 & -3.89 \\
\hline Spain & Netherlands & 1998 & 2001 & 1.57 & 2.12 & 0.68 & 5.39 & 0.08 & -1.29 \\
\hline Spain & Netherlands & 1999 & 2002 & 0.75 & 0.99 & 0.78 & 6.02 & 0.09 & -0.90 \\
\hline Spain & Portugal & 1995 & 1998 & -0.69 & -1.53 & 0.91 & 20.68 & 0.06 & -0.64 \\
\hline Spain & Portugal & 1996 & 1999 & 1.03 & 3.61 & 0.71 & 21.34 & 0.05 & -2.40 \\
\hline Spain & Portugal & 1997 & 2000 & 2.61 & 18.82 & 0.47 & 24.16 & 0.13 & -0.84 \\
\hline Spain & Portugal & 1998 & 2001 & 1.69 & 6.52 & 0.65 & 15.00 & 0.13 & -2.10 \\
\hline Spain & Portugal & 1999 & 2002 & 0.44 & 2.89 & 0.88 & 32.60 & 0.63 & -3.27 \\
\hline Spain & Australia & 1995 & 1998 & -2.62 & -8.15 & 1.30 & 35.00 & 0.33 & -1.85 \\
\hline Spain & Australia & 1996 & 1999 & -3.22 & -6.60 & 1.33 & 20.91 & 0.17 & -1.09 \\
\hline Spain & Australia & 1997 & 2000 & -0.29 & -0.20 & 0.87 & 4.30 & 0.03 & -1.49 \\
\hline Spain & Australia & 1998 & 2001 & 1.39 & 1.69 & 0.60 & 5.07 & 0.06 & -2.08 \\
\hline Spain & Australia & 1999 & 2002 & 0.38 & 0.59 & 0.72 & 7.66 & 0.10 & -0.35 \\
\hline Spain & Canada & 1995 & 1998 & 1.00 & 0.67 & 1.12 & 5.09 & 0.05 & -0.24 \\
\hline Spain & Canada & 1996 & 1999 & 12.80 & 4.76 & -0.96 & -2.24 & 0.03 & -3.50 \\
\hline Spain & Canada & 1997 & 2000 & 9.50 & 11.16 & -0.55 & -4.34 & 0.06 & -1.28 \\
\hline Spain & Canada & 1998 & 2001 & 4.64 & 6.91 & 0.13 & 1.34 & 0.05 & -1.40 \\
\hline Spain & Canada & 1999 & 2002 & 3.63 & 7.99 & 0.26 & 3.77 & 0.06 & -0.55 \\
\hline Spain & Japan & 1995 & 1998 & 0.06 & 0.03 & 3.00 & 4.53 & 0.05 & -0.42 \\
\hline Spain & Japan & 1996 & 1999 & -25.75 & -4.69 & 12.71 & 5.94 & 0.06 & -2.51 \\
\hline Spain & Japan & 1997 & 2000 & -8.22 & -3.92 & 5.61 & 6.71 & 0.10 & -2.40 \\
\hline Spain & Japan & 1998 & 2001 & -0.17 & -0.09 & 2.33 & 3.00 & 0.07 & -0.91 \\
\hline Spain & Japan & 1999 & 2002 & -8.14 & -2.10 & 5.61 & 3.47 & 0.14 & -1.29 \\
\hline Spain & Switzerland & 1995 & 1998 & -11.45 & -19.67 & 4.11 & 34.37 & 0.23 & -1.90 \\
\hline Spain & Switzerland & 1996 & 1999 & -10.86 & -16.64 & 3.98 & 27.22 & 0.20 & -1.53 \\
\hline Spain & Switzerland & 1997 & 2000 & -6.63 & -12.29 & 2.96 & 23.18 & 0.40 & -2.54 \\
\hline Spain & Switzerland & 1998 & 2001 & -4.34 & -5.15 & 2.38 & 11.74 & 0.24 & -0.89 \\
\hline Spain & Switzerland & 1999 & 2002 & -4.87 & -5.94 & 2.47 & 12.44 & 0.26 & -1.77 \\
\hline Spain & United Kingdom & 1995 & 1998 & -1.89 & -0.46 & 1.41 & 2.51 & 0.07 & -0.72 \\
\hline Spain & United Kingdom & 1996 & 1999 & -1.06 & -0.27 & 1.14 & 2.02 & 0.02 & -3.10 \\
\hline Spain & United Kingdom & 1997 & 2000 & -1.70 & -1.16 & 1.11 & 5.18 & 0.08 & -1.68 \\
\hline Spain & United Kingdom & 1998 & 2001 & 4.76 & 5.49 & 0.12 & 0.90 & 0.05 & -1.03 \\
\hline Spain & United Kingdom & 1999 & 2002 & 3.60 & 4.32 & 0.29 & 2.07 & 0.05 & -0.42 \\
\hline Spain & USA & 1995 & 1998 & -16.71 & -3.01 & 3.38 & 4.53 & 0.05 & -0.68 \\
\hline Spain & USA & 1996 & 1999 & -17.67 & -4.24 & 3.38 & 5.88 & 0.08 & -3.45 \\
\hline Spain & USA & 1997 & 2000 & -8.78 & -4.22 & 2.03 & 7.04 & 0.12 & -2.17 \\
\hline Spain & USA & 1998 & 2001 & 0.77 & 0.36 & 0.68 & 2.18 & 0.07 & -1.29 \\
\hline Spain & USA & 1999 & 2002 & 0.09 & 0.07 & 0.76 & 4.28 & 0.08 & -0.93 \\
\hline Australia & Austria & 1995 & 1998 & -5.44 & -5.03 & 2.07 & 12.98 & 0.11 & -1.46 \\
\hline Australia & Austria & 1996 & 1999 & -2.98 & -2.32 & 1.73 & 8.22 & 0.08 & -1.29 \\
\hline Australia & Austria & 1997 & 2000 & 3.07 & 3.73 & 0.68 & 4.85 & 0.13 & -1.35 \\
\hline Australia & Austria & 1998 & 2001 & 3.24 & 3.57 & 0.64 & 4.11 & 0.09 & -0.67 \\
\hline Australia & Austria & 1999 & 2002 & 1.57 & 1.81 & 0.93 & 6.13 & 0.10 & -1.12 \\
\hline Australia & Belgium & 1995 & 1998 & 0.05 & 0.05 & 1.36 & 9.75 & 0.11 & -1.73 \\
\hline Australia & Belgium & 1996 & 1999 & -3.22 & -2.28 & 1.92 & 7.66 & 0.20 & -1.39 \\
\hline Australia & Belgium & 1997 & 2000 & 3.55 & 6.51 & 0.61 & 6.43 & 0.17 & -3.40 \\
\hline Australia & Belgium & 1998 & 2001 & 3.64 & 6.99 & 0.56 & 6.39 & 0.16 & -0.58 \\
\hline Australia & Belgium & 1999 & 2002 & 3.26 & 4.61 & 0.60 & 5.13 & 0.11 & -1.24 \\
\hline Australia & Finland & 1995 & 1998 & 0.33 & 0.47 & 1.26 & 11.98 & 0.09 & -1.20 \\
\hline Australia & Finland & 1996 & 1999 & -1.08 & -1.22 & 1.55 & 9.83 & 0.10 & -1.21 \\
\hline Australia & Finland & 1997 & 2000 & 3.47 & 5.86 & 0.66 & 6.04 & 0.18 & -2.42 \\
\hline Australia & Finland & 1998 & 2001 & 3.16 & 6.57 & 0.71 & 7.95 & 0.18 & -1.89 \\
\hline Australia & Finland & 1999 & 2002 & 2.96 & 7.75 & 0.77 & 10.36 & 0.23 & -1.40 \\
\hline Australia & France & 1995 & 1998 & -4.33 & -6.58 & 1.58 & 19.68 & 0.32 & -1.83 \\
\hline Australia & France & 1996 & 1999 & -0.55 & -0.77 & 1.11 & 11.43 & 0.17 & -1.62 \\
\hline Australia & France & 1997 & 2000 & 4.53 & 6.15 & 0.37 & 3.42 & 0.13 & -1.00 \\
\hline Australia & France & 1998 & 2001 & 2.68 & 2.44 & 0.65 & 3.90 & 0.15 & -1.41 \\
\hline Australia & France & 1999 & 2002 & -0.41 & -0.45 & 1.15 & 7.91 & 0.30 & -1.93 \\
\hline Australia & Germany & 1995 & 1998 & -1.80 & -1.74 & 1.63 & 10.08 & 0.11 & -1.74 \\
\hline Australia & Germany & 1996 & 1999 & -1.90 & -1.50 & 1.65 & 7.48 & 0.12 & -2.01 \\
\hline Australia & Germany & 1997 & 2000 & 3.19 & 5.68 & 0.67 & 6.87 & 0.23 & -2.42 \\
\hline Australia & Germany & 1998 & 2001 & 2.81 & 5.25 & 0.73 & 7.78 & 0.25 & -1.41 \\
\hline Australia & Germany & 1999 & 2002 & 2.80 & 4.04 & 0.72 & 5.89 & 0.17 & -1.54 \\
\hline Australia & Ireland & 1995 & 1998 & 4.26 & 1.11 & 0.59 & 1.12 & 0.02 & -0.99 \\
\hline Australia & Ireland & 1996 & 1999 & 5.40 & 4.24 & 0.33 & 1.70 & 0.03 & -2.55 \\
\hline Australia & Ireland & 1997 & 2000 & 7.23 & 16.77 & -0.03 & -0.47 & 0.10 & -1.54 \\
\hline Australia & Ireland & 1998 & 2001 & 7.05 & 14.81 & -0.02 & -0.21 & 0.09 & -0.40 \\
\hline Australia & Ireland & 1999 & 2002 & 4.35 & 6.44 & 0.50 & 3.75 & 0.11 & -1.45 \\
\hline Australia & Italy & 1995 & 1998 & 1.15 & 2.18 & 0.66 & 14.23 & 0.11 & -1.35 \\
\hline Australia & Italy & 1996 & 1999 & 3.80 & 10.23 & 0.40 & 10.52 & 0.07 & -1.36 \\
\hline
\end{tabular}




\begin{tabular}{|c|c|c|c|c|c|c|c|c|c|}
\hline & national interest rate $\mathrm{r}$ & sample & eriod & & coin & gration & egression: & $=a+b$ & \\
\hline$\overline{\mathrm{r}_{1}}$ & $\mathrm{r}_{2}$ & start & $\overline{\text { end }}$ & $\mathrm{a}$ & t-stat(a) & $\mathrm{b}$ & t-stat(b) & DW & DF \\
\hline Australia & Italy & 1997 & 2000 & 6.71 & 21.58 & 0.04 & 1.08 & 0.10 & -1.09 \\
\hline Australia & Italy & 1998 & 2001 & 6.88 & 12.16 & 0.01 & 0.13 & 0.08 & -0.44 \\
\hline Australia & Italy & 1999 & 2002 & 2.87 & 2.86 & 0.66 & 4.01 & 0.10 & -1.19 \\
\hline Australia & Netherlands & 1995 & 1998 & -2.42 & -1.84 & 1.70 & 8.40 & 0.10 & -1.37 \\
\hline Australia & Netherlands & 1996 & 1999 & -2.69 & -1.60 & 1.74 & 6.11 & 0.11 & -1.89 \\
\hline Australia & Netherlands & 1997 & 2000 & 2.67 & 4.10 & 0.74 & 6.70 & 0.21 & -2.76 \\
\hline Australia & Netherlands & 1998 & 2001 & 1.86 & 3.23 & 0.87 & 8.88 & 0.25 & -1.73 \\
\hline Australia & Netherlands & 1999 & 2002 & 1.81 & 2.42 & 0.86 & 6.76 & 0.17 & -1.45 \\
\hline Australia & Portugal & 1995 & 1998 & 1.92 & 4.17 & 0.66 & 14.72 & 0.10 & -0.78 \\
\hline Australia & Portugal & 1996 & 1999 & 3.93 & 10.54 & 0.44 & 10.13 & 0.08 & -1.46 \\
\hline Australia & Portugal & 1997 & 2000 & 6.35 & 21.34 & 0.10 & 2.39 & 0.10 & -0.84 \\
\hline Australia & Portugal & 1998 & 2001 & 5.26 & 9.10 & 0.29 & 2.95 & 0.08 & -1.20 \\
\hline Australia & Portugal & 1999 & 2002 & 2.91 & 5.83 & 0.72 & 8.01 & 0.14 & -1.38 \\
\hline Australia & Spain & 1995 & 1998 & 2.26 & 12.19 & 0.74 & 35.00 & 0.34 & -2.02 \\
\hline Australia & Spain & 1996 & 1999 & 2.91 & 12.64 & 0.68 & 20.91 & 0.19 & -1.29 \\
\hline Australia & Spain & 1997 & 2000 & 5.11 & 11.29 & 0.33 & 4.30 & 0.11 & -0.68 \\
\hline Australia & Spain & 1998 & 2001 & 3.62 & 5.49 & 0.60 & 5.07 & 0.10 & -1.28 \\
\hline Australia & Spain & 1999 & 2002 & 2.72 & 5.00 & 0.78 & 7.66 & 0.13 & -1.09 \\
\hline Australia & Canada & 1995 & 1998 & 2.82 & 2.53 & 0.86 & 5.20 & 0.06 & -0.52 \\
\hline Australia & Canada & 1996 & 1999 & 8.77 & 4.35 & -0.20 & -0.62 & 0.03 & -2.84 \\
\hline Australia & Canada & 1997 & 2000 & 5.70 & 9.68 & 0.20 & 2.28 & 0.11 & -2.64 \\
\hline Australia & Canada & 1998 & 2001 & 3.29 & 7.83 & 0.54 & 8.79 & 0.24 & -1.62 \\
\hline Australia & Canada & 1999 & 2002 & 3.93 & 12.45 & 0.45 & 9.47 & 0.23 & -1.63 \\
\hline Australia & Japan & 1995 & 1998 & 2.55 & 1.76 & 2.14 & 4.17 & 0.05 & -0.56 \\
\hline Australia & Japan & 1996 & 1999 & -8.09 & -1.73 & 6.10 & 3.35 & 0.05 & -2.27 \\
\hline Australia & Japan & 1997 & 2000 & 8.35 & 4.63 & -0.53 & -0.73 & 0.10 & -1.62 \\
\hline Australia & Japan & 1998 & 2001 & 7.97 & 3.82 & -0.41 & -0.49 & 0.09 & -0.40 \\
\hline Australia & Japan & 1999 & 2002 & -5.47 & -1.31 & 5.15 & 2.96 & 0.12 & -1.71 \\
\hline Australia & Switzerland & 1995 & 1998 & -5.94 & -7.85 & 2.99 & 19.24 & 0.22 & -1.60 \\
\hline Australia & Switzerland & 1996 & 1999 & -3.91 & -4.27 & 2.58 & 12.56 & 0.15 & -1.23 \\
\hline Australia & Switzerland & 1997 & 2000 & 2.73 & 2.74 & 1.02 & 4.33 & 0.12 & -0.93 \\
\hline Australia & Switzerland & 1998 & 2001 & 2.04 & 1.34 & 1.18 & 3.22 & 0.09 & -0.28 \\
\hline Australia & Switzerland & 1999 & 2002 & 1.36 & 0.86 & 1.33 & 3.47 & 0.09 & -1.06 \\
\hline Australia & United Kingdom & 1995 & 1998 & 2.28 & 0.71 & 0.85 & 1.96 & 0.06 & -0.81 \\
\hline Australia & United Kingdom & 1996 & 1999 & 5.84 & 2.01 & 0.25 & 0.59 & 0.03 & -2.75 \\
\hline Australia & United Kingdom & 1997 & 2000 & 6.77 & 5.99 & 0.04 & 0.24 & 0.10 & -1.39 \\
\hline Australia & United Kingdom & 1998 & 2001 & 6.34 & 7.27 & 0.09 & 0.70 & 0.08 & -0.60 \\
\hline Australia & United Kingdom & 1999 & 2002 & 3.46 & 4.59 & 0.57 & 4.54 & 0.12 & -1.24 \\
\hline Australia & USA & 1995 & 1998 & -8.79 & -2.02 & 2.33 & 3.97 & 0.05 & -0.81 \\
\hline Australia & USA & 1996 & 1999 & -6.88 & -2.07 & 1.99 & 4.35 & 0.07 & -3.22 \\
\hline Australia & USA & 1997 & 2000 & -1.57 & -1.18 & 1.19 & 6.45 & 0.20 & -1.22 \\
\hline Australia & USA & 1998 & 2001 & -5.16 & -3.56 & 1.72 & 8.37 & 0.32 & -2.11 \\
\hline Australia & USA & 1999 & 2002 & -0.05 & -0.04 & 1.00 & 6.25 & 0.15 & -1.10 \\
\hline Canada & Austria & 1995 & 1998 & -2.36 & -2.40 & 1.34 & 9.24 & 0.20 & -2.10 \\
\hline Canada & Austria & 1996 & 1999 & 8.89 & 10.60 & -0.43 & -3.16 & 0.25 & -1.94 \\
\hline Canada & Austria & 1997 & 2000 & 9.35 & 5.98 & -0.46 & -1.72 & 0.09 & -0.94 \\
\hline Canada & Austria & 1998 & 2001 & 5.82 & 3.74 & 0.17 & 0.65 & 0.09 & 0.64 \\
\hline Canada & Austria & 1999 & 2002 & 2.12 & 1.06 & 0.77 & 2.20 & 0.06 & -0.43 \\
\hline Canada & Belgium & 1995 & 1998 & 0.24 & 0.46 & 1.03 & 12.25 & 0.28 & -1.82 \\
\hline Canada & Belgium & 1996 & 1999 & 5.23 & 5.44 & 0.18 & 1.07 & 0.19 & -1.14 \\
\hline Canada & Belgium & 1997 & 2000 & 0.97 & 1.15 & 1.00 & 6.74 & 0.17 & -2.35 \\
\hline Canada & Belgium & 1998 & 2001 & 3.81 & 3.98 & 0.51 & 3.17 & 0.09 & 1.09 \\
\hline Canada & Belgium & 1999 & 2002 & 2.45 & 1.66 & 0.67 & 2.76 & 0.06 & -0.56 \\
\hline Canada & Finland & 1995 & 1998 & 0.99 & 1.86 & 0.87 & 10.85 & 0.19 & -2.29 \\
\hline Canada & Finland & 1996 & 1999 & 7.30 & 10.50 & -0.19 & -1.51 & 0.23 & -1.56 \\
\hline Canada & Finland & 1997 & 2000 & 6.26 & 4.94 & 0.08 & 0.33 & 0.08 & -1.43 \\
\hline Canada & Finland & 1998 & 2001 & 3.34 & 3.48 & 0.65 & 3.64 & 0.09 & 0.01 \\
\hline Canada & Finland & 1999 & 2002 & 0.94 & 1.01 & 1.09 & 5.98 & 0.08 & -0.92 \\
\hline Canada & France & 1995 & 1998 & 1.53 & 1.26 & 0.63 & 4.25 & 0.10 & -2.69 \\
\hline Canada & France & 1996 & 1999 & 8.16 & 14.21 & -0.26 & -3.36 & 0.26 & -2.02 \\
\hline Canada & France & 1997 & 2000 & 11.53 & 10.47 & -0.71 & -4.43 & 0.13 & -0.90 \\
\hline Canada & France & 1998 & 2001 & 4.24 & 2.32 & 0.39 & 1.41 & 0.09 & 0.46 \\
\hline Canada & France & 1999 & 2002 & -2.81 & -1.32 & 1.46 & 4.36 & 0.09 & -0.77 \\
\hline Canada & Germany & 1995 & 1998 & -0.28 & -0.35 & 1.10 & 8.62 & 0.15 & -1.16 \\
\hline Canada & Germany & 1996 & 1999 & 7.02 & 8.19 & -0.13 & -0.90 & 0.22 & -1.28 \\
\hline Canada & Germany & 1997 & 2000 & 3.28 & 2.79 & 0.59 & 2.90 & 0.08 & -1.86 \\
\hline Canada & Germany & 1998 & 2001 & 1.53 & 1.68 & 0.94 & 5.85 & 0.11 & 1.03 \\
\hline Canada & Germany & 1999 & 2002 & 0.22 & 0.16 & 1.10 & 4.63 & 0.05 & -1.02 \\
\hline Canada & Ireland & 1995 & 1998 & -1.80 & -0.73 & 1.18 & 3.45 & 0.16 & -3.62 \\
\hline Canada & Ireland & 1996 & 1999 & 8.26 & 15.86 & -0.31 & -3.90 & 0.30 & -2.09 \\
\hline Canada & Ireland & 1997 & 2000 & 9.65 & 18.37 & -0.49 & -5.78 & 0.19 & -1.64 \\
\hline Canada & Ireland & 1998 & 2001 & 7.60 & 10.97 & -0.14 & -1.14 & 0.10 & 0.86 \\
\hline Canada & Ireland & 1999 & 2002 & 3.53 & 2.69 & 0.58 & 2.27 & 0.06 & -0.62 \\
\hline Canada & Italy & 1995 & 1998 & 4.61 & 5.66 & 0.18 & 2.55 & 0.09 & -3.22 \\
\hline Canada & Italy & 1996 & 1999 & 7.23 & 26.30 & -0.11 & -3.72 & 0.26 & -2.05 \\
\hline Canada & Italy & 1997 & 2000 & 9.23 & 29.12 & -0.34 & -8.35 & 0.20 & -1.36 \\
\hline Canada & Italy & 1998 & 2001 & 7.72 & 9.37 & -0.14 & -1.10 & 0.10 & 0.85 \\
\hline Canada & Italy & 1999 & 2002 & 3.31 & 1.63 & 0.52 & 1.57 & 0.06 & -0.53 \\
\hline Canada & Netherlands & 1995 & 1998 & -1.33 & -1.49 & 1.24 & 9.05 & 0.16 & -1.46 \\
\hline Canada & Netherlands & 1996 & 1999 & 7.59 & 7.47 & -0.23 & -1.32 & 0.23 & -1.35 \\
\hline Canada & Netherlands & 1997 & 2000 & 3.65 & 2.61 & 0.51 & 2.18 & 0.08 & -1.68 \\
\hline Canada & Netherlands & 1998 & 2001 & 0.75 & 0.70 & 1.04 & 5.69 & 0.13 & 0.13 \\
\hline Canada & Netherlands & 1999 & 2002 & -0.69 & -0.44 & 1.23 & 4.57 & 0.07 & -0.84 \\
\hline Canada & Portugal & 1995 & 1998 & 4.40 & 6.27 & 0.22 & 3.29 & 0.09 & -2.62 \\
\hline Canada & Portugal & 1996 & 1999 & 7.34 & 28.51 & -0.13 & -4.40 & 0.28 & -2.23 \\
\hline Canada & Portugal & 1997 & 2000 & 9.02 & 25.56 & -0.34 & -6.89 & 0.16 & -0.84 \\
\hline Canada & Portugal & 1998 & 2001 & 6.17 & 6.67 & 0.11 & 0.71 & 0.09 & 0.47 \\
\hline
\end{tabular}




\begin{tabular}{|c|c|c|c|c|c|c|c|c|c|}
\hline & national interest rate $\mathrm{r}$ & sample & eriod & & coin & gration & regression: & $=\mathrm{a}+\mathrm{b}$ & \\
\hline $\mathrm{r}_{1}$ & $\mathrm{r}_{2}$ & start & end & $\mathrm{a}$ & t-stat(a) & b & t-stat(b) & DW & DF \\
\hline Canada & Portugal & 1999 & 2002 & 1.87 & 1.56 & 0.84 & 3.88 & 0.08 & -0.79 \\
\hline Canada & Spain & 1995 & 1998 & 3.94 & 7.13 & 0.32 & 5.09 & 0.11 & -2.88 \\
\hline Canada & Spain & 1996 & 1999 & 6.95 & 21.47 & -0.10 & -2.24 & 0.22 & -1.70 \\
\hline Canada & Spain & 1997 & 2000 & 9.76 & 13.55 & -0.53 & -4.34 & 0.11 & -0.54 \\
\hline Canada & Spain & 1998 & 2001 & 5.23 & 4.39 & 0.29 & 1.34 & 0.09 & 0.42 \\
\hline Canada & Spain & 1999 & 2002 & 1.67 & 1.29 & 0.91 & 3.77 & 0.07 & -0.77 \\
\hline Canada & Australia & 1995 & 1998 & 2.97 & 4.13 & 0.43 & 5.20 & 0.11 & -2.75 \\
\hline Canada & Australia & 1996 & 1999 & 6.57 & 12.76 & -0.04 & -0.62 & 0.20 & -1.29 \\
\hline Canada & Australia & 1997 & 2000 & 3.10 & 1.97 & 0.51 & 2.28 & 0.09 & -2.59 \\
\hline Canada & Australia & 1998 & 2001 & -1.29 & -1.40 & 1.17 & 8.79 & 0.25 & -1.00 \\
\hline Canada & Australia & 1999 & 2002 & -3.52 & -3.32 & 1.46 & 9.47 & 0.21 & -1.25 \\
\hline Canada & Japan & 1995 & 1998 & -0.11 & -0.17 & 2.42 & 10.32 & 0.36 & -2.17 \\
\hline Canada & Japan & 1996 & 1999 & 15.00 & 7.48 & -3.41 & -4.36 & 0.34 & -2.22 \\
\hline Canada & Japan & 1997 & 2000 & 20.95 & 10.54 & -5.69 & -7.19 & 0.29 & -1.81 \\
\hline Canada & Japan & 1998 & 2001 & 9.31 & 3.04 & -1.01 & -0.81 & 0.09 & 0.81 \\
\hline Canada & Japan & 1999 & 2002 & -8.34 & -1.06 & 6.18 & 1.89 & 0.09 & -0.74 \\
\hline Canada & Switzerland & 1995 & 1998 & 0.56 & 0.42 & 1.26 & 4.58 & 0.10 & -2.62 \\
\hline Canada & Switzerland & 1996 & 1999 & 9.19 & 11.97 & -0.66 & -3.85 & 0.27 & -2.22 \\
\hline Canada & Switzerland & 1997 & 2000 & 13.78 & 8.80 & -1.69 & -4.55 & 0.12 & -0.56 \\
\hline Canada & Switzerland & 1998 & 2001 & 8.09 & 3.26 & -0.31 & -0.51 & 0.09 & 0.64 \\
\hline Canada & Switzerland & 1999 & 2002 & 3.75 & 1.18 & 0.66 & 0.86 & 0.06 & -0.42 \\
\hline Canada & United Kingdom & 1995 & 1998 & -3.67 & -2.03 & 1.41 & 5.74 & 0.45 & -3.12 \\
\hline Canada & United Kingdom & 1996 & 1999 & 9.46 & 7.58 & -0.46 & -2.57 & 0.28 & -1.50 \\
\hline Canada & United Kingdom & 1997 & 2000 & 11.70 & 7.12 & -0.74 & -3.07 & 0.14 & -1.58 \\
\hline Canada & United Kingdom & 1998 & 2001 & 3.57 & 2.97 & 0.50 & 2.72 & 0.09 & -0.36 \\
\hline Canada & United Kingdom & 1999 & 2002 & -3.01 & -3.69 & 1.60 & 11.70 & 0.33 & -2.04 \\
\hline Canada & USA & 1995 & 1998 & 1.71 & 0.49 & 0.66 & 1.41 & 0.07 & -2.87 \\
\hline Canada & USA & 1996 & 1999 & 13.53 & 9.33 & -1.00 & -5.02 & 0.39 & -1.88 \\
\hline Canada & USA & 1997 & 2000 & 9.27 & 3.18 & -0.36 & -0.89 & 0.09 & -1.23 \\
\hline Canada & USA & 1998 & 2001 & -13.13 & -7.79 & 2.83 & 11.85 & 0.35 & -1.48 \\
\hline Canada & USA & 1999 & 2002 & -9.29 & -6.81 & 2.29 & 11.59 & 0.22 & -1.48 \\
\hline Japan & Austria & 1995 & 1998 & -0.30 & -0.89 & 0.46 & 9.11 & 0.23 & -2.49 \\
\hline Japan & Austria & 1996 & 1999 & 1.69 & 24.26 & 0.14 & 12.54 & 0.30 & -2.10 \\
\hline Japan & Austria & 1997 & 2000 & 1.55 & 10.36 & 0.16 & 6.38 & 0.15 & -1.10 \\
\hline Japan & Austria & 1998 & 2001 & 1.90 & 11.56 & 0.09 & 3.35 & 0.15 & -1.75 \\
\hline Japan & Austria & 1999 & 2002 & 2.16 & 25.78 & 0.04 & 2.92 & 0.46 & -3.20 \\
\hline Japan & Belgium & 1995 & 1998 & 0.62 & 3.21 & 0.35 & 11.51 & 0.38 & -2.42 \\
\hline Japan & Belgium & 1996 & 1999 & 2.17 & 15.18 & 0.07 & 2.75 & 0.08 & 0.48 \\
\hline Japan & Belgium & 1997 & 2000 & 2.85 & 19.64 & -0.06 & -2.34 & 0.11 & -1.32 \\
\hline Japan & Belgium & 1998 & 2001 & 2.74 & 23.49 & -0.05 & -2.46 & 0.18 & -1.97 \\
\hline Japan & Belgium & 1999 & 2002 & 2.39 & 34.56 & 0.00 & 0.18 & 0.41 & -2.88 \\
\hline Japan & Finland & 1995 & 1998 & 1.02 & 4.62 & 0.27 & 8.18 & 0.21 & -2.57 \\
\hline Japan & Finland & 1996 & 1999 & 1.95 & 28.92 & 0.11 & 9.08 & 0.17 & -1.38 \\
\hline Japan & Finland & 1997 & 2000 & 2.00 & 13.95 & 0.10 & 3.60 & 0.09 & -1.03 \\
\hline Japan & Finland & 1998 & 2001 & 2.17 & 17.92 & 0.05 & 2.35 & 0.14 & -1.77 \\
\hline Japan & Finland & 1999 & 2002 & 2.24 & 45.89 & 0.03 & 3.27 & 0.48 & -3.31 \\
\hline Japan & France & 1995 & 1998 & 0.95 & 2.31 & 0.23 & 4.49 & 0.13 & -2.94 \\
\hline Japan & France & 1996 & 1999 & 2.04 & 31.35 & 0.07 & 8.15 & 0.16 & -1.16 \\
\hline Japan & France & 1997 & 2000 & 1.68 & 14.61 & 0.12 & 7.26 & 0.18 & -1.51 \\
\hline Japan & France & 1998 & 2001 & 1.76 & 9.08 & 0.11 & 3.61 & 0.18 & -1.63 \\
\hline Japan & France & 1999 & 2002 & 2.10 & 20.77 & 0.05 & 2.99 & 0.47 & -3.27 \\
\hline Japan & Germany & 1995 & 1998 & 0.23 & 0.94 & 0.41 & 10.57 & 0.29 & -1.95 \\
\hline Japan & Germany & 1996 & 1999 & 2.05 & 17.98 & 0.09 & 4.53 & 0.11 & 0.21 \\
\hline Japan & Germany & 1997 & 2000 & 2.56 & 15.72 & -0.01 & -0.34 & 0.08 & -1.26 \\
\hline Japan & Germany & 1998 & 2001 & 2.62 & 18.90 & -0.03 & -1.23 & 0.15 & -1.87 \\
\hline Japan & Germany & 1999 & 2002 & 2.42 & 33.93 & 0.00 & -0.37 & 0.41 & -2.80 \\
\hline Japan & Ireland & 1995 & 1998 & -0.24 & -0.28 & 0.42 & 3.62 & 0.16 & -4.04 \\
\hline Japan & Ireland & 1996 & 1999 & 1.97 & 55.72 & 0.09 & 16.96 & 0.53 & -2.66 \\
\hline Japan & Ireland & 1997 & 2000 & 1.95 & 66.07 & 0.09 & 19.06 & 0.69 & -3.26 \\
\hline Japan & Ireland & 1998 & 2001 & 1.97 & 49.87 & 0.08 & 12.43 & 0.60 & -2.90 \\
\hline Japan & Ireland & 1999 & 2002 & 2.16 & 44.69 & 0.05 & 5.05 & 0.63 & -3.37 \\
\hline Japan & Italy & 1995 & 1998 & 2.02 & 7.28 & 0.07 & 2.84 & 0.11 & -3.41 \\
\hline Japan & Italy & 1996 & 1999 & 2.31 & 75.47 & 0.03 & 8.70 & 0.17 & -1.26 \\
\hline Japan & Italy & 1997 & 2000 & 2.15 & 63.08 & 0.05 & 10.83 & 0.26 & -1.92 \\
\hline Japan & Italy & 1998 & 2001 & 1.94 & 32.12 & 0.08 & 8.69 & 0.37 & -2.27 \\
\hline Japan & Italy & 1999 & 2002 & 2.14 & 26.07 & 0.04 & 3.18 & 0.48 & -3.17 \\
\hline Japan & Netherlands & 1995 & 1998 & -0.20 & -0.82 & 0.47 & 12.02 & 0.33 & -2.26 \\
\hline Japan & Netherlands & 1996 & 1999 & 1.87 & 14.60 & 0.12 & 5.42 & 0.14 & 0.14 \\
\hline Japan & Netherlands & 1997 & 2000 & 2.44 & 13.03 & 0.01 & 0.39 & 0.08 & -1.22 \\
\hline Japan & Netherlands & 1998 & 2001 & 2.57 & 15.75 & -0.02 & -0.72 & 0.14 & -1.84 \\
\hline Japan & Netherlands & 1999 & 2002 & 2.37 & 28.78 & 0.00 & 0.32 & 0.41 & -2.90 \\
\hline Japan & Portugal & 1995 & 1998 & 1.86 & 8.11 & 0.09 & 4.18 & 0.12 & -2.81 \\
\hline Japan & Portugal & 1996 & 1999 & 2.32 & 73.65 & 0.03 & 7.96 & 0.16 & -1.12 \\
\hline Japan & Portugal & 1997 & 2000 & 2.18 & 53.31 & 0.05 & 8.22 & 0.19 & -1.69 \\
\hline Japan & Portugal & 1998 & 2001 & 1.96 & 24.29 & 0.08 & 6.19 & 0.25 & -1.76 \\
\hline Japan & Portugal & 1999 & 2002 & 2.18 & 42.86 & 0.04 & 4.23 & 0.55 & -3.44 \\
\hline Japan & Spain & 1995 & 1998 & 1.93 & 9.71 & 0.10 & 4.53 & 0.13 & -3.08 \\
\hline Japan & Spain & 1996 & 1999 & 2.33 & 57.28 & 0.03 & 5.94 & 0.12 & -0.84 \\
\hline Japan & Spain & 1997 & 2000 & 1.99 & 25.63 & 0.09 & 6.71 & 0.15 & -1.47 \\
\hline Japan & Spain & 1998 & 2001 & 2.06 & 15.83 & 0.07 & 3.00 & 0.16 & -1.68 \\
\hline Japan & Spain & 1999 & 2002 & 2.20 & 38.64 & 0.04 & 3.47 & 0.51 & -3.40 \\
\hline Japan & Australia & 1995 & 1998 & 1.70 & 6.36 & 0.13 & 4.17 & 0.12 & -2.89 \\
\hline Japan & Australia & 1996 & 1999 & 2.32 & 31.59 & 0.03 & 3.35 & 0.09 & -0.24 \\
\hline Japan & Australia & 1997 & 2000 & 2.66 & 12.62 & -0.02 & -0.73 & 0.08 & -1.40 \\
\hline Japan & Australia & 1998 & 2001 & 2.54 & 14.30 & -0.01 & -0.49 & 0.14 & -1.75 \\
\hline Japan & Australia & 1999 & 2002 & 2.18 & 30.10 & 0.03 & 2.96 & 0.46 & -3.30 \\
\hline Japan & Canada & 1995 & 1998 & 0.87 & 4.63 & 0.29 & 10.32 & 0.37 & -2.27 \\
\hline
\end{tabular}




\begin{tabular}{|c|c|c|c|c|c|c|c|c|c|}
\hline & national interest rate $r$ & sample & eriod & & coin & egration $\mathrm{r}$ & regression & $=\mathrm{a}+\mathrm{b}$ & \\
\hline $\mathrm{r}_{1}$ & $r_{2}$ & start & end & $\mathrm{a}$ & t-stat(a) & $\mathrm{b}$ & t-stat(b) & DW & $\mathrm{DF}$ \\
\hline Japan & Canada & 1996 & 1999 & 3.10 & 25.07 & -0.09 & -4.36 & 0.20 & -1.64 \\
\hline Japan & Canada & 1997 & 2000 & 3.13 & 35.95 & -0.09 & -7.19 & 0.29 & -1.73 \\
\hline Japan & Canada & 1998 & 2001 & 2.55 & 21.57 & -0.01 & -0.81 & 0.14 & -1.66 \\
\hline Japan & Canada & 1999 & 2002 & 2.32 & 57.17 & 0.01 & 1.89 & 0.45 & -3.03 \\
\hline Japan & Switzerland & 1995 & 1998 & 0.57 & 1.27 & 0.46 & 4.98 & 0.13 & -2.87 \\
\hline Japan & Switzerland & 1996 & 1999 & 1.90 & 18.98 & 0.15 & 6.60 & 0.14 & -1.16 \\
\hline Japan & Switzerland & 1997 & 2000 & 1.46 & 7.91 & 0.25 & 5.68 & 0.16 & -1.33 \\
\hline Japan & Switzerland & 1998 & 2001 & 2.11 & 7.34 & 0.08 & 1.18 & 0.14 & -1.71 \\
\hline Japan & Switzerland & 1999 & 2002 & 2.03 & 15.88 & 0.09 & 2.92 & 0.50 & -3.31 \\
\hline Japan & United Kingdom & 1995 & 1998 & -1.29 & -2.35 & 0.56 & 7.46 & 0.74 & -4.08 \\
\hline Japan & United Kingdom & 1996 & 1999 & 1.52 & 10.50 & 0.15 & 7.23 & 0.20 & -0.78 \\
\hline Japan & United Kingdom & 1997 & 2000 & 1.39 & 8.64 & 0.16 & 6.96 & 0.22 & -1.48 \\
\hline Japan & United Kingdom & 1998 & 2001 & 1.83 & 15.17 & 0.10 & 5.18 & 0.23 & -1.83 \\
\hline Japan & United Kingdom & 1999 & 2002 & 2.36 & 33.52 & 0.01 & 0.54 & 0.41 & -2.88 \\
\hline Japan & USA & 1995 & 1998 & -1.74 & -1.67 & 0.61 & 4.35 & 0.12 & -2.67 \\
\hline Japan & USA & 1996 & 1999 & 1.50 & 6.27 & 0.15 & 4.44 & 0.12 & -0.43 \\
\hline Japan & USA & 1997 & 2000 & 2.04 & 5.52 & 0.07 & 1.27 & 0.08 & -1.13 \\
\hline Japan & USA & 1998 & 2001 & 2.88 & 7.32 & -0.06 & -1.08 & 0.15 & -1.80 \\
\hline Japan & USA & 1999 & 2002 & 2.26 & 19.58 & 0.02 & 1.16 & 0.42 & -2.95 \\
\hline Switzerland & Austria & 1995 & 1998 & 0.26 & 0.90 & 0.68 & 16.06 & 0.10 & -1.46 \\
\hline Switzerland & Austria & 1996 & 1999 & 0.26 & 0.86 & 0.69 & 13.86 & 0.14 & -1.25 \\
\hline Switzerland & Austria & 1997 & 2000 & 1.33 & 4.18 & 0.49 & 9.10 & 0.14 & -1.78 \\
\hline Switzerland & Austria & 1998 & 2001 & 1.96 & 9.47 & 0.38 & 10.66 & 0.25 & -1.38 \\
\hline Switzerland & Austria & 1999 & 2002 & 1.71 & 9.60 & 0.42 & 13.56 & 0.40 & -1.93 \\
\hline Switzerland & Belgium & 1995 & 1998 & 2.15 & 7.81 & 0.43 & 9.88 & 0.14 & -1.62 \\
\hline Switzerland & Belgium & 1996 & 1999 & 1.55 & 2.65 & 0.51 & 4.94 & 0.12 & -0.36 \\
\hline Switzerland & Belgium & 1997 & 2000 & 4.17 & 10.52 & 0.01 & 0.12 & 0.04 & -2.23 \\
\hline Switzerland & Belgium & 1998 & 2001 & 3.20 & 14.68 & 0.16 & 4.43 & 0.12 & -1.29 \\
\hline Switzerland & Belgium & 1999 & 2002 & 2.87 & 12.02 & 0.21 & 5.29 & 0.15 & -1.21 \\
\hline Switzerland & Finland & 1995 & 1998 & 2.20 & 10.73 & 0.41 & 13.05 & 0.08 & -0.94 \\
\hline Switzerland & Finland & 1996 & 1999 & 1.35 & 5.07 & 0.55 & 11.69 & 0.12 & -0.93 \\
\hline Switzerland & Finland & 1997 & 2000 & 2.35 & 7.48 & 0.35 & 5.99 & 0.09 & -2.44 \\
\hline Switzerland & Finland & 1998 & 2001 & 2.80 & 15.68 & 0.25 & 7.62 & 0.18 & -0.58 \\
\hline Switzerland & Finland & 1999 & 2002 & 2.89 & 19.17 & 0.24 & 8.25 & 0.20 & -1.51 \\
\hline Switzerland & France & 1995 & 1998 & 0.60 & 6.17 & 0.52 & 44.06 & 0.68 & -3.04 \\
\hline Switzerland & France & 1996 & 1999 & 1.31 & 12.46 & 0.43 & 30.07 & 0.45 & -2.28 \\
\hline Switzerland & France & 1997 & 2000 & 1.49 & 9.20 & 0.40 & 16.87 & 0.46 & -2.39 \\
\hline Switzerland & France & 1998 & 2001 & 1.78 & 5.99 & 0.36 & 8.05 & 0.26 & -1.66 \\
\hline Switzerland & France & 1999 & 2002 & 1.48 & 5.20 & 0.42 & 9.36 & 0.31 & -1.72 \\
\hline Switzerland & Germany & 1995 & 1998 & 1.43 & 4.94 & 0.54 & 11.87 & 0.14 & -2.09 \\
\hline Switzerland & Germany & 1996 & 1999 & 1.28 & 2.89 & 0.55 & 7.18 & 0.13 & -0.81 \\
\hline Switzerland & Germany & 1997 & 2000 & 3.31 & 8.27 & 0.16 & 2.27 & 0.06 & -2.14 \\
\hline Switzerland & Germany & 1998 & 2001 & 3.51 & 12.54 & 0.11 & 2.31 & 0.09 & -1.02 \\
\hline Switzerland & Germany & 1999 & 2002 & 3.41 & 11.55 & 0.13 & 2.44 & 0.10 & -0.78 \\
\hline Switzerland & Ireland & 1995 & 1998 & 2.56 & 2.17 & 0.32 & 1.94 & 0.03 & -1.19 \\
\hline Switzerland & Ireland & 1996 & 1999 & 2.86 & 7.51 & 0.24 & 4.18 & 0.04 & -2.00 \\
\hline Switzerland & Ireland & 1997 & 2000 & 3.26 & 18.41 & 0.16 & 5.51 & 0.10 & -2.04 \\
\hline Switzerland & Ireland & 1998 & 2001 & 3.69 & 23.31 & 0.08 & 2.98 & 0.08 & -0.75 \\
\hline Switzerland & Ireland & 1999 & 2002 & 2.73 & 16.80 & 0.28 & 8.69 & 0.34 & -2.39 \\
\hline Switzerland & Italy & 1995 & 1998 & 2.41 & 18.49 & 0.22 & 18.98 & 0.20 & -2.42 \\
\hline Switzerland & Italy & 1996 & 1999 & 2.96 & 52.93 & 0.16 & 27.47 & 0.31 & -2.33 \\
\hline Switzerland & Italy & 1997 & 2000 & 3.28 & 39.19 & 0.12 & 11.64 & 0.13 & -0.34 \\
\hline Switzerland & Italy & 1998 & 2001 & 3.46 & 19.54 & 0.11 & 4.01 & 0.08 & -0.91 \\
\hline Switzerland & Italy & 1999 & 2002 & 1.66 & 10.58 & 0.40 & 15.79 & 0.86 & -3.64 \\
\hline Switzerland & Netherlands & 1995 & 1998 & 1.12 & 3.12 & 0.58 & 10.44 & 0.13 & -1.61 \\
\hline Switzerland & Netherlands & 1996 & 1999 & 0.74 & 1.36 & 0.63 & 6.83 & 0.14 & -0.90 \\
\hline Switzerland & Netherlands & 1997 & 2000 & 2.88 & 6.52 & 0.23 & 3.03 & 0.07 & -2.35 \\
\hline Switzerland & Netherlands & 1998 & 2001 & 3.24 & 10.24 & 0.16 & 2.90 & 0.10 & -0.97 \\
\hline Switzerland & Netherlands & 1999 & 2002 & 3.18 & 9.53 & 0.16 & 2.85 & 0.11 & -0.81 \\
\hline Switzerland & Portugal & 1995 & 1998 & 2.60 & 35.22 & 0.22 & 31.20 & 0.18 & -1.01 \\
\hline Switzerland & Portugal & 1996 & 1999 & 2.98 & 68.24 & 0.18 & 34.78 & 0.28 & -2.38 \\
\hline Switzerland & Portugal & 1997 & 2000 & 3.19 & 55.59 & 0.15 & 18.42 & 0.26 & -1.29 \\
\hline Switzerland & Portugal & 1998 & 2001 & 3.04 & 19.25 & 0.19 & 7.11 & 0.14 & -1.02 \\
\hline Switzerland & Portugal & 1999 & 2002 & 2.59 & 19.85 & 0.28 & 11.92 & 0.34 & -2.06 \\
\hline Switzerland & Spain & 1995 & 1998 & 2.86 & 48.15 & 0.23 & 34.37 & 0.23 & -2.01 \\
\hline Switzerland & Spain & 1996 & 1999 & 2.83 & 46.01 & 0.24 & 27.22 & 0.21 & -1.23 \\
\hline Switzerland & Spain & 1997 & 2000 & 2.40 & 30.20 & 0.31 & 23.18 & 0.42 & -2.38 \\
\hline Switzerland & Spain & 1998 & 2001 & 2.41 & 16.09 & 0.32 & 11.74 & 0.25 & -0.88 \\
\hline Switzerland & Spain & 1999 & 2002 & 2.47 & 18.36 & 0.31 & 12.44 & 0.29 & -1.87 \\
\hline Switzerland & Australia & 1995 & 1998 & 2.30 & 17.13 & 0.30 & 19.24 & 0.22 & -1.50 \\
\hline Switzerland & Australia & 1996 & 1999 & 2.17 & 11.89 & 0.30 & 12.56 & 0.14 & -0.64 \\
\hline Switzerland & Australia & 1997 & 2000 & 2.22 & 4.80 & 0.28 & 4.33 & 0.07 & -1.23 \\
\hline Switzerland & Australia & 1998 & 2001 & 3.07 & 9.11 & 0.16 & 3.22 & 0.08 & -0.76 \\
\hline Switzerland & Australia & 1999 & 2002 & 3.06 & 9.89 & 0.16 & 3.47 & 0.09 & -0.46 \\
\hline Switzerland & Canada & 1995 & 1998 & 3.18 & 8.66 & 0.25 & 4.58 & 0.05 & 0.01 \\
\hline Switzerland & Canada & 1996 & 1999 & 6.73 & 11.21 & -0.37 & -3.85 & 0.08 & -2.79 \\
\hline Switzerland & Canada & 1997 & 2000 & 5.44 & 20.00 & -0.18 & -4.55 & 0.09 & -0.89 \\
\hline Switzerland & Canada & 1998 & 2001 & 4.28 & 17.20 & -0.02 & -0.51 & 0.07 & -0.99 \\
\hline Switzerland & Canada & 1999 & 2002 & 3.97 & 21.64 & 0.02 & 0.86 & 0.08 & -0.45 \\
\hline Switzerland & Japan & 1995 & 1998 & 2.71 & 6.26 & 0.76 & 4.98 & 0.06 & -0.46 \\
\hline Switzerland & Japan & 1996 & 1999 & -3.96 & -3.11 & 3.28 & 6.60 & 0.09 & -2.06 \\
\hline Switzerland & Japan & 1997 & 2000 & 0.05 & 0.07 & 1.66 & 5.68 & 0.12 & -1.81 \\
\hline Switzerland & Japan & 1998 & 2001 & 3.28 & 4.38 & 0.36 & 1.18 & 0.07 & -0.85 \\
\hline Switzerland & Japan & 1999 & 2002 & -0.04 & -0.02 & 1.74 & 2.92 & 0.16 & -1.28 \\
\hline Switzerland & United Kingdom & 1995 & 1998 & 2.05 & 2.13 & 0.38 & 2.89 & 0.11 & -1.02 \\
\hline Switzerland & United Kingdom & 1996 & 1999 & 2.39 & 2.53 & 0.30 & 2.16 & 0.03 & -1.66 \\
\hline Switzerland & United Kingdom & 1997 & 2000 & 2.46 & 4.58 & 0.26 & 3.29 & 0.08 & -1.40 \\
\hline
\end{tabular}




\begin{tabular}{|c|c|c|c|c|c|c|c|c|c|}
\hline & national interest rate $r$ & sample & eriod & & coir & ggration & egression: & $=a+b$ & \\
\hline $\mathrm{r}_{1}$ & $\mathrm{r}_{2}$ & start & end & $\mathrm{a}$ & t-stat(a) & $\mathrm{b}$ & t-stat(b) & DW & DF \\
\hline $\begin{array}{l}\text { Switzerland } \\
\end{array}$ & United Kingdom & 1998 & 2001 & 4.81 & 15.87 & -0.10 & -2.18 & 0.08 & -1.33 \\
\hline Switzerland & United Kingdom & 1999 & 2002 & 4.23 & 13.66 & -0.02 & -0.33 & 0.07 & -0.20 \\
\hline Switzerland & USA & 1995 & 1998 & -2.21 & -1.83 & 0.95 & 5.84 & 0.09 & -1.01 \\
\hline Switzerland & USA & 1996 & 1999 & -2.22 & -2.43 & 0.92 & 7.28 & 0.14 & -2.72 \\
\hline Switzerland & USA & 1997 & 2000 & -0.44 & -0.64 & 0.64 & 6.78 & 0.13 & -1.71 \\
\hline Switzerland & USA & 1998 & 2001 & 3.76 & 4.51 & 0.06 & 0.48 & 0.07 & -0.92 \\
\hline Switzerland & USA & 1999 & 2002 & 3.12 & 6.34 & 0.15 & 2.04 & 0.08 & -0.75 \\
\hline United Kingdom & Austria & 1995 & 1998 & 4.26 & 6.99 & 0.46 & 5.08 & 0.75 & -3.23 \\
\hline United Kingdom & Austria & 1996 & 1999 & 4.13 & 7.17 & 0.46 & 4.82 & 0.18 & -1.98 \\
\hline United Kingdom & Austria & 1997 & 2000 & 2.96 & 4.26 & 0.66 & 5.55 & 0.17 & -0.37 \\
\hline United Kingdom & Austria & 1998 & 2001 & 5.29 & 4.64 & 0.20 & 1.01 & 0.08 & -0.47 \\
\hline United Kingdom & Austria & 1999 & 2002 & 5.16 & 4.57 & 0.14 & 0.70 & 0.08 & -0.25 \\
\hline United Kingdom & Belgium & 1995 & 1998 & 5.19 & 13.41 & 0.34 & 5.61 & 0.78 & -3.30 \\
\hline United Kingdom & Belgium & 1996 & 1999 & 5.16 & 7.39 & 0.31 & 2.50 & 0.15 & -1.49 \\
\hline United Kingdom & Belgium & 1997 & 2000 & 6.60 & 9.86 & 0.04 & 0.33 & 0.11 & -1.05 \\
\hline United Kingdom & Belgium & 1998 & 2001 & 7.39 & 9.64 & -0.16 & -1.24 & 0.11 & -0.66 \\
\hline United Kingdom & Belgium & 1999 & 2002 & 4.18 & 5.09 & 0.29 & 2.17 & 0.07 & -0.38 \\
\hline United Kingdom & Finland & 1995 & 1998 & 5.48 & 14.97 & 0.28 & 5.14 & 0.71 & -3.13 \\
\hline United Kingdom & Finland & 1996 & 1999 & 5.11 & 10.76 & 0.32 & 3.78 & 0.16 & -1.90 \\
\hline United Kingdom & Finland & 1997 & 2000 & 4.52 & 7.28 & 0.43 & 3.73 & 0.14 & -0.47 \\
\hline United Kingdom & Finland & 1998 & 2001 & 5.19 & 6.62 & 0.23 & 1.61 & 0.08 & -0.64 \\
\hline United Kingdom & Finland & 1999 & 2002 & 3.82 & 6.40 & 0.42 & 3.58 & 0.09 & -0.75 \\
\hline United Kingdom & France & 1995 & 1998 & 5.82 & 9.42 & 0.19 & 2.46 & 0.54 & -2.79 \\
\hline United Kingdom & France & 1996 & 1999 & 5.48 & 12.39 & 0.19 & 3.25 & 0.16 & -1.70 \\
\hline United Kingdom & France & 1997 & 2000 & 3.50 & 6.40 & 0.49 & 6.08 & 0.22 & -1.13 \\
\hline United Kingdom & France & 1998 & 2001 & 3.86 & 2.92 & 0.39 & 1.95 & 0.10 & -0.49 \\
\hline United Kingdom & France & 1999 & 2002 & 2.78 & 2.15 & 0.50 & 2.45 & 0.11 & -0.63 \\
\hline United Kingdom & Germany & 1995 & 1998 & 4.83 & 10.19 & 0.40 & 5.33 & 0.74 & -3.21 \\
\hline United Kingdom & Germany & 1996 & 1999 & 4.57 & 8.11 & 0.41 & 4.14 & 0.17 & -1.61 \\
\hline United Kingdom & Germany & 1997 & 2000 & 5.14 & 7.69 & 0.29 & 2.52 & 0.11 & -0.79 \\
\hline United Kingdom & Germany & 1998 & 2001 & 5.13 & 5.93 & 0.23 & 1.53 & 0.08 & -0.46 \\
\hline United Kingdom & Germany & 1999 & 2002 & 2.65 & 3.55 & 0.58 & 4.42 & 0.08 & -0.97 \\
\hline United Kingdom & Ireland & 1995 & 1998 & 1.86 & 1.91 & 0.76 & 5.63 & 0.81 & -4.03 \\
\hline United Kingdom & Ireland & 1996 & 1999 & 4.74 & 14.49 & 0.33 & 6.69 & 0.28 & -1.52 \\
\hline United Kingdom & Ireland & 1997 & 2000 & 5.09 & 17.82 & 0.28 & 6.14 & 0.22 & -1.38 \\
\hline United Kingdom & Ireland & 1998 & 2001 & 4.77 & 10.52 & 0.29 & 3.76 & 0.11 & -0.64 \\
\hline United Kingdom & Ireland & 1999 & 2002 & 5.63 & 7.52 & 0.06 & 0.42 & 0.08 & -0.26 \\
\hline United Kingdom & Italy & 1995 & 1998 & 6.73 & 17.27 & 0.05 & 1.56 & 0.49 & -2.71 \\
\hline United Kingdom & Italy & 1996 & 1999 & 6.19 & 28.97 & 0.08 & 3.45 & 0.17 & -1.67 \\
\hline United Kingdom & Italy & 1997 & 2000 & 5.50 & 27.99 & 0.17 & 6.91 & 0.23 & -1.48 \\
\hline United Kingdom & Italy & 1998 & 2001 & 4.38 & 8.22 & 0.31 & 3.91 & 0.12 & -0.38 \\
\hline United Kingdom & Italy & 1999 & 2002 & 5.56 & 4.93 & 0.06 & 0.34 & 0.08 & -0.25 \\
\hline United Kingdom & Netherlands & 1995 & 1998 & 4.33 & 8.44 & 0.47 & 5.87 & 0.78 & -3.31 \\
\hline United Kingdom & Netherlands & 1996 & 1999 & 3.83 & 5.91 & 0.52 & 4.76 & 0.19 & -1.74 \\
\hline United Kingdom & Netherlands & 1997 & 2000 & 4.72 & 6.23 & 0.36 & 2.78 & 0.11 & -0.72 \\
\hline United Kingdom & Netherlands & 1998 & 2001 & 4.92 & 4.89 & 0.26 & 1.52 & 0.08 & -0.51 \\
\hline United Kingdom & Netherlands & 1999 & 2002 & 2.43 & 2.74 & 0.60 & 3.97 & 0.08 & -0.63 \\
\hline United Kingdom & Portugal & 1995 & 1998 & 6.57 & 19.37 & 0.08 & 2.30 & 0.53 & -2.75 \\
\hline United Kingdom & Portugal & 1996 & 1999 & 6.24 & 29.52 & 0.08 & 3.26 & 0.17 & -1.64 \\
\hline United Kingdom & Portugal & 1997 & 2000 & 5.66 & 25.85 & 0.17 & 5.46 & 0.19 & -1.13 \\
\hline United Kingdom & Portugal & 1998 & 2001 & 4.74 & 7.44 & 0.29 & 2.69 & 0.10 & -0.49 \\
\hline United Kingdom & Portugal & 1999 & 2002 & 4.63 & 6.40 & 0.24 & 1.83 & 0.09 & -0.47 \\
\hline United Kingdom & Spain & 1995 & 1998 & 6.61 & 22.24 & 0.09 & 2.51 & 0.54 & -2.78 \\
\hline United Kingdom & Spain & 1996 & 1999 & 6.41 & 25.66 & 0.07 & 2.02 & 0.15 & -1.55 \\
\hline United Kingdom & Spain & 1997 & 2000 & 4.87 & 12.83 & 0.33 & 5.18 & 0.17 & -0.83 \\
\hline United Kingdom & Spain & 1998 & 2001 & 5.65 & 6.36 & 0.14 & 0.90 & 0.09 & -0.52 \\
\hline United Kingdom & Spain & 1999 & 2002 & 4.38 & 5.73 & 0.30 & 2.07 & 0.09 & -0.55 \\
\hline United Kingdom & Australia & 1995 & 1998 & 6.56 & 16.38 & 0.09 & 1.96 & 0.52 & -2.68 \\
\hline United Kingdom & Australia & 1996 & 1999 & 6.67 & 16.95 & 0.03 & 0.59 & 0.14 & -1.34 \\
\hline United Kingdom & Australia & 1997 & 2000 & 6.60 & 7.12 & 0.03 & 0.24 & 0.11 & -1.04 \\
\hline United Kingdom & Australia & 1998 & 2001 & 5.67 & 5.09 & 0.11 & 0.70 & 0.09 & -0.73 \\
\hline United Kingdom & Australia & 1999 & 2002 & 2.23 & 2.72 & 0.54 & 4.54 & 0.13 & -0.62 \\
\hline United Kingdom & Canada & 1995 & 1998 & 5.36 & 15.35 & 0.30 & 5.74 & 0.85 & -3.40 \\
\hline United Kingdom & Canada & 1996 & 1999 & 8.59 & 12.97 & -0.27 & -2.57 & 0.22 & -1.58 \\
\hline United Kingdom & Canada & 1997 & 2000 & 8.35 & 16.54 & -0.23 & -3.07 & 0.18 & -1.39 \\
\hline United Kingdom & Canada & 1998 & 2001 & 4.57 & 6.58 & 0.27 & 2.72 & 0.09 & -1.53 \\
\hline United Kingdom & Canada & 1999 & 2002 & 2.90 & 11.01 & 0.47 & 11.70 & 0.35 & -1.98 \\
\hline United Kingdom & Japan & 1995 & 1998 & 4.58 & 12.31 & 0.98 & 7.46 & 1.13 & -4.28 \\
\hline United Kingdom & Japan & 1996 & 1999 & -2.11 & -1.69 & 3.52 & 7.23 & 0.27 & -1.62 \\
\hline United Kingdom & Japan & 1997 & 2000 & -1.03 & -0.92 & 3.13 & 6.96 & 0.26 & -1.45 \\
\hline United Kingdom & Japan & 1998 & 2001 & -2.92 & -1.61 & 3.82 & 5.18 & 0.19 & -0.91 \\
\hline United Kingdom & Japan & 1999 & 2002 & 3.58 & 0.81 & 0.99 & 0.54 & 0.08 & -0.25 \\
\hline United Kingdom & Switzerland & 1995 & 1998 & 5.37 & 7.89 & 0.40 & 2.89 & 0.57 & -2.83 \\
\hline United Kingdom & Switzerland & 1996 & 1999 & 5.51 & 8.58 & 0.31 & 2.16 & 0.15 & -1.55 \\
\hline United Kingdom & Switzerland & 1997 & 2000 & 3.70 & 3.91 & 0.74 & 3.29 & 0.15 & -0.84 \\
\hline United Kingdom & Switzerland & 1998 & 2001 & 10.25 & 5.86 & -0.92 & -2.18 & 0.10 & -0.91 \\
\hline United Kingdom & Switzerland & 1999 & 2002 & 6.51 & 3.76 & -0.14 & -0.33 & 0.08 & -0.08 \\
\hline United Kingdom & USA & 1995 & 1998 & 2.78 & 1.85 & 0.61 & 3.03 & 0.56 & -2.81 \\
\hline United Kingdom & USA & 1996 & 1999 & 2.37 & 1.97 & 0.62 & 3.75 & 0.18 & -1.50 \\
\hline United Kingdom & USA & 1997 & 2000 & 2.67 & 1.75 & 0.57 & 2.72 & 0.13 & -0.96 \\
\hline United Kingdom & USA & 1998 & 2001 & 0.27 & 0.11 & 0.88 & 2.65 & 0.09 & -0.94 \\
\hline United Kingdom & USA & 1999 & 2002 & -3.01 & -4.82 & 1.30 & 14.36 & 0.41 & -2.30 \\
\hline USA & Austria & 1995 & 1998 & 5.60 & 13.09 & 0.27 & 4.32 & 0.14 & -1.06 \\
\hline USA & Austria & 1996 & 1999 & 5.04 & 11.45 & 0.36 & 5.04 & 0.17 & -1.90 \\
\hline USA & Austria & 1997 & 2000 & 5.13 & 10.36 & 0.36 & 4.24 & 0.12 & -1.27 \\
\hline USA & Austria & 1998 & 2001 & 6.31 & 13.51 & 0.13 & 1.59 & 0.14 & -0.91 \\
\hline USA & Austria & 1999 & 2002 & 4.89 & 6.68 & 0.35 & 2.74 & 0.06 & 0.34 \\
\hline
\end{tabular}




\begin{tabular}{|c|c|c|c|c|c|c|c|c|c|}
\hline & national interest rate $r$ & & sample & eriod & & coir & ggration & egression: & $=a+b$ \\
\hline $\mathrm{r}_{1}$ & $\mathrm{r}_{2}$ & & start & end & $\mathrm{a}$ & t-stat(a) & $\mathrm{b}$ & t-stat(b) & DW \\
\hline USA & Belgium & & 1995 & 1998 & 6.19 & 22.23 & 0.20 & 4.52 & 0.11 \\
\hline USA & Belgium & & 1996 & 1999 & 5.16 & 10.58 & 0.37 & 4.33 & 0.12 \\
\hline USA & Belgium & & 1997 & 2000 & 5.81 & 15.26 & 0.25 & 3.73 & 0.09 \\
\hline USA & Belgium & & 1998 & 2001 & 5.74 & 22.18 & 0.22 & 5.11 & 0.19 \\
\hline USA & Belgium & & 1999 & 2002 & 4.98 & 9.43 & 0.32 & 3.66 & 0.06 \\
\hline USA & Finland & & 1995 & 1998 & 6.49 & 23.92 & 0.15 & 3.54 & 0.12 \\
\hline USA & Finland & & 1996 & 1999 & 5.69 & 16.12 & 0.28 & 4.46 & 0.14 \\
\hline USA & Finland & & 1997 & 2000 & 5.51 & 14.39 & 0.32 & 4.50 & 0.12 \\
\hline USA & Finland & & 1998 & 2001 & 5.85 & 20.65 & 0.23 & 4.27 & 0.15 \\
\hline USA & Finland & & 1999 & 2002 & 4.73 & 13.79 & 0.42 & 6.36 & 0.09 \\
\hline USA & France & & 1995 & 1998 & 5.53 & 16.50 & 0.23 & 5.71 & 0.19 \\
\hline USA & France & & 1996 & 1999 & 5.39 & 20.73 & 0.26 & 7.25 & 0.23 \\
\hline USA & France & & 1997 & 2000 & 4.90 & 14.82 & 0.34 & 7.05 & 0.20 \\
\hline USA & France & & 1998 & 2001 & 5.65 & 10.58 & 0.21 & 2.63 & 0.16 \\
\hline USA & France & & 1999 & 2002 & 3.13 & 4.01 & 0.59 & 4.84 & 0.11 \\
\hline USA & Germany & & 1995 & 1998 & 5.57 & 19.18 & 0.30 & 6.48 & 0.14 \\
\hline USA & Germany & & 1996 & 1999 & 4.67 & 13.67 & 0.45 & 7.59 & 0.20 \\
\hline USA & Germany & & 1997 & 2000 & 4.93 & 15.64 & 0.40 & 7.27 & 0.18 \\
\hline USA & Germany & & 1998 & 2001 & 4.96 & 24.78 & 0.37 & 10.46 & 0.37 \\
\hline USA & Germany & & 1999 & 2002 & 4.27 & 8.82 & 0.46 & 5.47 & 0.08 \\
\hline USA & Ireland & & 1995 & 1998 & 6.73 & 8.05 & 0.10 & 0.84 & 0.10 \\
\hline USA & Ireland & & 1996 & 1999 & 6.39 & 19.22 & 0.13 & 2.63 & 0.11 \\
\hline USA & Ireland & & 1997 & 2000 & 6.99 & 28.23 & 0.04 & 0.93 & 0.08 \\
\hline USA & Ireland & & 1998 & 2001 & 7.31 & 34.49 & -0.05 & -1.24 & 0.15 \\
\hline USA & Ireland & & 1999 & 2002 & 5.80 & 11.69 & 0.22 & 2.23 & 0.07 \\
\hline USA & Italy & & 1995 & 1998 & 6.24 & 31.66 & 0.11 & 6.18 & 0.18 \\
\hline USA & Italy & & 1996 & 1999 & 6.33 & 53.15 & 0.10 & 8.16 & 0.23 \\
\hline USA & Italy & & 1997 & 2000 & 6.53 & 43.78 & 0.09 & 4.74 & 0.12 \\
\hline USA & Italy & & 1998 & 2001 & 7.08 & 27.69 & 0.00 & -0.12 & 0.13 \\
\hline USA & Italy & & 1999 & 2002 & 5.20 & 6.97 & 0.28 & 2.29 & 0.08 \\
\hline USA & Netherlands & & 1995 & 1998 & 5.34 & 16.19 & 0.32 & 6.38 & 0.12 \\
\hline USA & Netherlands & & 1996 & 1999 & 4.12 & 10.23 & 0.53 & 7.80 & 0.20 \\
\hline USA & Netherlands & & 1997 & 2000 & 4.48 & 13.08 & 0.46 & 8.04 & 0.19 \\
\hline USA & Netherlands & & 1998 & 2001 & 4.68 & 19.03 & 0.41 & 9.69 & 0.35 \\
\hline USA & Netherlands & & 1999 & 2002 & 4.08 & 7.00 & 0.48 & 4.87 & 0.08 \\
\hline USA & Portugal & & 1995 & 1998 & 6.26 & 39.10 & 0.12 & 7.49 & 0.20 \\
\hline USA & Portugal & & 1996 & 1999 & 6.31 & 57.95 & 0.12 & 9.09 & 0.23 \\
\hline USA & Portugal & & 1997 & 2000 & 6.41 & 47.61 & 0.12 & 6.19 & 0.14 \\
\hline USA & Portugal & & 1998 & 2001 & 6.66 & 23.86 & 0.07 & 1.41 & 0.13 \\
\hline USA & Portugal & & 1999 & 2002 & 5.09 & 11.38 & 0.33 & 4.06 & 0.07 \\
\hline USA & Spain & & 1995 & 1998 & 6.66 & 37.95 & 0.09 & 4.53 & 0.14 \\
\hline USA & Spain & & 1996 & 1999 & 6.39 & 41.77 & 0.13 & 5.88 & 0.16 \\
\hline USA & Spain & & 1997 & 2000 & 5.72 & 26.62 & 0.26 & 7.04 & 0.17 \\
\hline USA & Spain & & 1998 & 2001 & 6.28 & 17.71 & 0.14 & 2.18 & 0.15 \\
\hline USA & Spain & & 1999 & 2002 & 4.90 & 10.42 & 0.38 & 4.28 & 0.09 \\
\hline USA & Australia & & 1995 & 1998 & 6.50 & 27.10 & 0.11 & 3.97 & 0.13 \\
\hline USA & Australia & & 1996 & 1999 & 6.15 & 23.82 & 0.15 & 4.35 & 0.13 \\
\hline USA & Australia & & 1997 & 2000 & 4.41 & 10.13 & 0.40 & 6.45 & 0.18 \\
\hline USA & Australia & & 1998 & 2001 & 4.61 & 15.74 & 0.35 & 8.37 & 0.37 \\
\hline USA & Australia & & 1999 & 2002 & 3.75 & 7.42 & 0.46 & 6.25 & 0.13 \\
\hline USA & Canada & & 1995 & 1998 & 7.02 & 23.52 & 0.06 & 1.41 & 0.09 \\
\hline USA & Canada & & 1996 & 1999 & 9.47 & 21.41 & -0.35 & -5.02 & 0.28 \\
\hline USA & Canada & & 1997 & 2000 & 7.53 & 21.14 & -0.05 & -0.89 & 0.08 \\
\hline USA & Canada & & 1998 & 2001 & 5.23 & 33.91 & 0.27 & 11.85 & 0.39 \\
\hline USA & Canada & & 1999 & 2002 & 4.79 & 25.87 & 0.33 & 11.59 & 0.21 \\
\hline USA & Japan & & 1995 & 1998 & 6.10 & 19.64 & 0.48 & 4.35 & 0.13 \\
\hline USA & Japan & & 1996 & 1999 & 2.00 & 1.69 & 2.05 & 4.44 & 0.14 \\
\hline USA & Japan & & 1997 & 2000 & 5.91 & 5.73 & 0.52 & 1.27 & 0.08 \\
\hline USA & Japan & & 1998 & 2001 & 8.05 & 8.63 & -0.41 & -1.08 & 0.14 \\
\hline USA & Japan & & 1999 & 2002 & 3.38 & 1.12 & 1.47 & 1.16 & 0.07 \\
\hline USA & Switzerland & & 1995 & 1998 & 5.26 & 14.07 & 0.45 & 5.84 & 0.17 \\
\hline USA & Switzerland & & 1996 & 1999 & 4.67 & 13.07 & 0.58 & 7.28 & 0.21 \\
\hline USA & Switzerland & & 1997 & 2000 & 3.95 & 8.18 & 0.78 & 6.78 & 0.16 \\
\hline USA & Switzerland & & 1998 & 2001 & 6.69 & 8.78 & 0.09 & 0.48 & 0.13 \\
\hline USA & Switzerland & & 1999 & 2002 & 4.54 & 3.93 & 0.57 & 2.04 & 0.06 \\
\hline USA & United Kingdom & & 1995 & 1998 & 5.44 & 8.25 & 0.27 & 3.03 & 0.18 \\
\hline USA & United Kingdom & & 1996 & 1999 & 4.66 & 6.73 & 0.38 & 3.75 & 0.13 \\
\hline USA & United Kingdom & & 1997 & 2000 & 5.57 & 9.19 & 0.24 & 2.72 & 0.10 \\
\hline USA & United Kingdom & & 1998 & 2001 & 6.07 & 16.44 & 0.15 & 2.65 & 0.14 \\
\hline USA & United Kingdom & & 1999 & 2002 & 3.16 & 12.04 & 0.63 & 14.36 & 0.38 \\
\hline Panel B & ition relative to rest of eurozon & & & & & & & & \\
\hline Austria & eurozone average excluding & Austria & 1995 & 1998 & 2.23 & 5.46 & 0.67 & 11.13 & 0.07 \\
\hline Austria & eurozone average excluding & Austria & 1996 & 1999 & 2.81 & 14.93 & 0.55 & 17.53 & 0.18 \\
\hline Austria & eurozone average excluding & Austria & 1997 & 2000 & 2.82 & 8.50 & 0.56 & 9.27 & 0.11 \\
\hline Austria & eurozone average excluding & Austria & 1998 & 2001 & 0.46 & 0.92 & 1.05 & 10.67 & 0.15 \\
\hline Austria & eurozone average excluding & Austria & 1999 & 2002 & -1.18 & -2.57 & 1.39 & 15.01 & 0.28 \\
\hline Belgium & eurozone average excluding & Belgium & 1995 & 1998 & 0.86 & 1.75 & 0.66 & 11.03 & 0.13 \\
\hline Belgium & eurozone average excluding & Belgium & 1996 & 1999 & 3.52 & 12.26 & 0.30 & 7.44 & 0.28 \\
\hline Belgium & eurozone average excluding & Belgium & 1997 & 2000 & 4.84 & 6.88 & 0.13 & 1.21 & 0.08 \\
\hline Belgium & eurozone average excluding & Belgium & 1998 & 2001 & 1.05 & 1.04 & 0.81 & 4.86 & 0.07 \\
\hline Belgium & eurozone average excluding & Belgium & 1999 & 2002 & -1.50 & -3.13 & 1.29 & 15.75 & 0.34 \\
\hline Finland & eurozone average excluding & Finland & 1995 & 1998 & 0.01 & 0.02 & 0.81 & 13.74 & 0.05 \\
\hline Finland & eurozone average excluding & Finland & 1996 & 1999 & 1.88 & 9.31 & 0.53 & 18.53 & 0.13 \\
\hline Finland & eurozone average excluding & Finland & 1997 & 2000 & 2.08 & 4.79 & 0.52 & 7.69 & 0.09 \\
\hline Finland & eurozone average excluding & Finland & 1998 & 2001 & -1.27 & -2.65 & 1.09 & 13.78 & 0.23 \\
\hline Finland & eurozone average excluding & Finland & 1999 & 2002 & -3.29 & -7.78 & 1.43 & 19.90 & 0.33 \\
\hline France & eurozone average excluding & France & 1995 & 1998 & 2.33 & 15.46 & 0.73 & 39.06 & 0.88 \\
\hline
\end{tabular}




\begin{tabular}{|c|c|c|c|c|c|c|c|c|c|c|c|c|c|c|}
\hline \multicolumn{3}{|c|}{ national interest rate $r$} & \multicolumn{2}{|c|}{ sample period } & \multicolumn{7}{|c|}{ cointegration regression: $r_{1}=a+b r_{2}$} & \multirow{2}{*}{$\begin{array}{l}\text { cointe- } \\
\text { gration? }\end{array}$} & \multicolumn{2}{|c|}{ ECM } \\
\hline $\mathrm{r}_{1}$ & $\mathrm{r}_{2}$ & & start & $\overline{\text { end }}$ & $\mathrm{a}$ & t-stat(a) & $\mathrm{b}$ & t-stat(b) & DW & DF & $\overline{\mathrm{ADF}}$ & & speed & t-stat \\
\hline France & eurozone average excluding & France & 1996 & 1999 & 1.98 & 13.56 & 0.77 & 37.21 & 0.77 & -3.38 & -4.70 & yes & -0.32 & -2.84 \\
\hline France & eurozone average excluding & France & 1997 & 2000 & 1.92 & 9.88 & 0.78 & 25.39 & 0.80 & -3.39 & -4.21 & yes & -0.57 & -4.47 \\
\hline France & eurozone average excluding & France & 1998 & 2001 & 2.06 & 7.26 & 0.76 & 15.91 & 0.80 & -3.37 & -3.57 & yes & -0.32 & -2.75 \\
\hline France & eurozone average excluding & France & 1999 & 2002 & 1.88 & 5.72 & 0.78 & 13.64 & 0.52 & -2.75 & -3.17 & yes & -0.30 & -2.89 \\
\hline Germany & eurozone average excluding & Germany & 1995 & 1998 & 2.05 & 5.53 & 0.49 & 11.69 & 0.13 & -4.24 & -3.46 & yes & -0.16 & -2.45 \\
\hline Germany & eurozone average excluding & Germany & 1996 & 1999 & 3.36 & 14.02 & 0.31 & 10.07 & 0.19 & -0.85 & -3.01 & no & -0.14 & -2.28 \\
\hline Germany & eurozone average excluding & Germany & 1997 & 2000 & 4.31 & 8.23 & 0.21 & 2.68 & 0.08 & -0.77 & -1.65 & no & -0.07 & -1.88 \\
\hline Germany & eurozone average excluding & Germany & 1998 & 2001 & 2.82 & 3.33 & 0.45 & 3.35 & 0.08 & -1.14 & -1.74 & no & -0.10 & -2.28 \\
\hline Germany & eurozone average excluding & Germany & 1999 & 2002 & 0.97 & 1.17 & 0.79 & 5.70 & 0.13 & -2.15 & -2.78 & no & -0.25 & -4.01 \\
\hline Ireland & eurozone average excluding & Ireland & 1995 & 1998 & 6.31 & 15.65 & 0.11 & 2.18 & 0.28 & -1.30 & -2.28 & no & -0.14 & -2.20 \\
\hline Ireland & eurozone average excluding & Ireland & 1996 & 1999 & 3.42 & 5.07 & 0.44 & 4.68 & 0.08 & -0.04 & -0.86 & no & 0.00 & -0.09 \\
\hline Ireland & eurozone average excluding & Ireland & 1997 & 2000 & -0.03 & -0.03 & 0.96 & 5.90 & 0.09 & -1.16 & -1.50 & no & -0.20 & -2.72 \\
\hline Ireland & eurozone average excluding & Ireland & 1998 & 2001 & -0.50 & -0.32 & 1.03 & 3.96 & 0.09 & -1.02 & -1.51 & no & -0.05 & -1.18 \\
\hline Ireland & eurozone average excluding & Ireland & 1999 & 2002 & 1.04 & 1.05 & 0.69 & 4.07 & 0.18 & -1.85 & -2.34 & no & -0.14 & -1.91 \\
\hline Italy & eurozone average excluding & Italy & 1995 & 1998 & -2.98 & -3.04 & 1.96 & 14.61 & 0.10 & -2.71 & -1.11 & no & -0.03 & -0.47 \\
\hline Italy & eurozone average excluding & Italy & 1996 & 1999 & -10.46 & -16.01 & 3.09 & 30.48 & 0.25 & -0.48 & -1.61 & no & -0.07 & -0.58 \\
\hline Italy & eurozone average excluding & Italy & 1997 & 2000 & -8.25 & -3.68 & 2.61 & 7.11 & 0.03 & -0.10 & -0.80 & no & -0.02 & -0.48 \\
\hline Italy & eurozone average excluding & Italy & 1998 & 2001 & 0.61 & 0.37 & 1.02 & 3.71 & 0.06 & -3.37 & -2.54 & yes & -0.23 & -3.63 \\
\hline Italy & eurozone average excluding & Italy & 1999 & 2002 & 1.75 & 2.94 & 0.75 & 7.30 & 0.27 & -2.07 & -2.17 & no & -0.14 & -1.74 \\
\hline Netherlands & eurozone average excluding & Netherlands & 1995 & 1998 & 2.32 & 6.72 & 0.51 & 12.07 & 0.13 & -2.85 & -2.05 & no & -0.13 & -1.88 \\
\hline Netherlands & eurozone average excluding & Netherlands & 1996 & 1999 & 3.51 & 16.43 & 0.34 & 11.35 & 0.27 & -1.28 & -2.41 & no & -0.20 & -2.64 \\
\hline Netherlands & eurozone average excluding & Netherlands & 1997 & 2000 & 3.44 & 7.14 & 0.38 & 5.15 & 0.08 & -0.82 & -1.40 & no & -0.16 & -3.16 \\
\hline Netherlands & eurozone average excluding & Netherlands & 1998 & 2001 & 1.32 & 2.11 & 0.74 & 7.26 & 0.09 & -1.47 & -1.93 & no & -0.26 & -3.62 \\
\hline Netherlands & eurozone average excluding & Netherlands & 1999 & 2002 & 0.20 & 0.40 & 0.96 & 11.44 & 0.17 & -1.65 & -3.14 & no & -0.23 & -3.46 \\
\hline Portugal & eurozone average excluding & Portugal & 1995 & 1998 & -4.26 & -8.66 & 1.80 & 29.50 & 0.13 & -1.26 & & no & -0.11 & -2.15 \\
\hline Portugal & eurozone average excluding & Portugal & 1996 & 1999 & -5.75 & -13.13 & 2.01 & 32.29 & 0.17 & -1.08 & -2.16 & no & -0.19 & -3.05 \\
\hline Portugal & eurozone average excluding & Portugal & 1997 & 2000 & -7.21 & -9.41 & 2.20 & 18.44 & 0.13 & -1.54 & -2.17 & no & -0.09 & -1.37 \\
\hline Portugal & eurozone average excluding & Portugal & 1998 & 2001 & -2.61 & -3.85 & 1.41 & 12.63 & 0.19 & -1.72 & & no & -0.11 & -1.14 \\
\hline Portugal & eurozone average excluding & Portugal & 1999 & 2002 & -2.04 & -3.31 & 1.29 & 12.30 & 0.20 & -2.34 & -2.59 & no & -0.16 & -1.90 \\
\hline Spain & eurozone average excluding & Spain & 1995 & 1998 & -6.09 & -11.33 & 1.82 & 27.32 & 0.13 & -0.86 & -1.71 & no & -0.07 & -1.34 \\
\hline Spain & eurozone average excluding & Spain & 1996 & 1999 & -3.68 & -7.83 & 1.50 & 22.67 & 0.10 & -2.50 & -2.10 & no & -0.07 & -1.30 \\
\hline Spain & eurozone average excluding & Spain & 1997 & 2000 & -1.12 & -3.39 & 1.08 & 21.23 & 0.18 & -2.19 & -2.12 & no & -0.06 & -0.77 \\
\hline Spain & eurozone average excluding & Spain & 1998 & 2001 & -0.63 & -1.36 & 1.01 & 13.33 & 0.18 & -1.61 & -2.70 & no & -0.01 & -0.13 \\
\hline Spain & eurozone average excluding & Spain & 1999 & 2002 & -1.92 & -3.80 & 1.22 & 14.35 & 0.21 & -1.94 & -2.55 & no & -0.08 & -0.81 \\
\hline Australia & eurozone average & & 1995 & 1998 & -1.40 & -2.76 & 1.24 & 19.87 & 0.15 & -0.72 & -1.55 & no & -0.10 & -1.73 \\
\hline Australia & eurozone average & & 1996 & 1999 & 1.10 & 2.00 & 0.93 & 11.94 & 0.09 & -1.52 & -2.11 & no & -0.10 & -1.89 \\
\hline Australia & eurozone average & & 1997 & 2000 & 4.98 & 8.59 & 0.32 & 3.56 & 0.11 & -0.81 & -0.96 & no & -0.06 & -1.53 \\
\hline Australia & eurozone average & & 1998 & 2001 & 3.06 & 3.66 & 0.64 & 4.66 & 0.11 & -1.57 & & no & -0.07 & -1.11 \\
\hline Australia & eurozone average & & 1999 & 2002 & 0.74 & 0.96 & 1.04 & 7.96 & 0.17 & -1.28 & & no & -0.11 & -1.66 \\
\hline Canada & eurozone average & & 1995 & 1998 & 2.32 & 2.57 & 0.54 & 4.87 & 0.10 & -2.47 & & no & -0.07 & -1.24 \\
\hline Canada & eurozone average & & 1996 & 1999 & 7.61 & 16.34 & -0.19 & -2.96 & 0.25 & -1.84 & -2.06 & no & -0.20 & -2.37 \\
\hline Canada & eurozone average & & 1997 & 2000 & 10.14 & 11.13 & -0.54 & -3.83 & 0.12 & -0.91 & -1.26 & no & -0.21 & -3.19 \\
\hline Canada & eurozone average & & 1998 & 2001 & 3.76 & 2.64 & 0.51 & 2.15 & 0.08 & 0.34 & -0.35 & no & 0.04 & 0.81 \\
\hline Canada & eurozone average & & 1999 & 2002 & -1.29 & -0.72 & 1.32 & 4.34 & 0.06 & -0.74 & & no & -0.03 & -0.93 \\
\hline Japan & eurozone average & & 1995 & 1998 & 1.24 & 4.05 & 0.19 & 5.18 & 0.14 & -2.79 & -4.32 & yes & -0.10 & -1.98 \\
\hline Japan & eurozone average & & 1996 & 1999 & 2.16 & 40.82 & 0.06 & 7.81 & 0.14 & -0.87 & & no & -0.05 & -0.76 \\
\hline Japan & eurozone average & & 1997 & 2000 & 1.87 & 19.74 & 0.10 & 6.73 & 0.14 & -1.32 & & no & -0.07 & -1.21 \\
\hline Japan & eurozone average & & 1998 & 2001 & 1.95 & 12.25 & 0.08 & 3.17 & 0.15 & -1.51 & & no & -0.12 & -1.90 \\
\hline Japan & eurozone average & & 1999 & 2002 & 2.23 & 24.96 & 0.03 & 1.86 & 0.43 & -3.15 & & yes & -0.25 & -2.41 \\
\hline Switzerland & eurozone average & & 1995 & 1998 & 1.59 & 16.92 & 0.40 & 34.96 & 0.34 & -1.60 & -2.65 & no & -0.18 & -1.96 \\
\hline Switzerland & eurozone average & & 1996 & 1999 & 2.01 & 18.78 & 0.35 & 22.96 & 0.22 & -1.49 & -2.32 & no & -0.09 & -1.24 \\
\hline Switzerland & eurozone average & & 1997 & 2000 & 2.14 & 13.72 & 0.33 & 13.41 & 0.21 & -1.52 & -2.46 & no & -0.13 & -1.87 \\
\hline Switzerland & eurozone average & & 1998 & 2001 & 2.44 & 9.12 & 0.28 & 6.42 & 0.13 & -1.03 & -1.66 & no & -0.04 & -0.74 \\
\hline Switzerland & eurozone average & & 1999 & 2002 & 2.07 & 7.67 & 0.35 & 7.65 & 0.19 & -1.50 & -2.28 & no & -0.19 & -2.35 \\
\hline United Kingdom & eurozone average & & 1995 & 1998 & 5.96 & 12.77 & 0.17 & 2.98 & 0.55 & -2.81 & & yes & -0.36 & -3.16 \\
\hline United Kingdom & eurozone average & & 1996 & 1999 & 5.71 & 16.53 & 0.17 & 3.49 & 0.16 & -1.70 & -2.28 & no & -0.11 & -1.82 \\
\hline United Kingdom & eurozone average & & 1997 & 2000 & 4.01 & 9.79 & 0.44 & 6.89 & 0.20 & -1.00 & -1.50 & no & -0.11 & -1.58 \\
\hline United Kingdom & eurozone average & & 1998 & 2001 & 3.61 & 3.53 & 0.47 & 2.79 & 0.09 & -0.28 & -0.81 & no & -0.06 & -1.33 \\
\hline United Kingdom & eurozone average & & 1999 & 2002 & 2.82 & 2.66 & 0.53 & 2.95 & 0.08 & -0.65 & & no & -0.11 & -1.88 \\
\hline USA & eurozone average & & 1995 & 1998 & 5.89 & 23.60 & 0.19 & 6.26 & 0.16 & -1.43 & -2.24 & no & -0.14 & -2.59 \\
\hline USA & eurozone average & & 1996 & 1999 & 5.72 & 29.33 & 0.22 & 8.00 & 0.21 & -2.48 & & no & -0.15 & -2.01 \\
\hline USA & eurozone average & & 1997 & 2000 & 5.26 & 21.69 & 0.31 & 8.15 & 0.18 & -1.49 & & no & -0.20 & -2.58 \\
\hline USA & eurozone average & & 1998 & 2001 & 5.51 & 13.82 & 0.25 & 3.87 & 0.16 & -1.24 & & no & -0.07 & -1.11 \\
\hline USA & eurozone average & & 1999 & 2002 & 3.59 & 5.61 & 0.56 & 5.19 & 0.08 & -0.30 & & no & -0.02 & -0.46 \\
\hline
\end{tabular}

Notes: Cointegration is based on the Durbin-Watson (DW), Dickey-Fuller (DF) and Augmented Dickey-Fuller (ADF) tests. The critical values for 100 observations at the 5\% and $10 \%$ significance levels are as follows: 0.386 and 0.322 for DW, -3.37 and $-3.03 \mathrm{DF}$ and -2.84 and -3.17 for ADF. Cointegration is considered to exist if at least two test statistics are significant at the $10 \%$ level or if at least one test-statistic is significant at the $5 \%$ level. For the error correction model (ECM) the speed of adjustment coefficient and its t-test statistic are reported. All t-tests statistics (t-stat) report tests of the estimated coefficients against zero. 


\begin{tabular}{|c|c|c|c|c|c|c|c|c|c|c|}
\hline & national interest rate $\mathrm{r}$ & sample & eriod & & coir & gration & egression & $=a+b$ & & \\
\hline $\mathrm{r}_{1}$ & $\mathrm{r}_{2}$ & start & end & $\mathrm{a}$ & t-stat(a) & $\mathrm{b}$ & t-stat(b) & DW & DF & $\mathrm{ADF}$ \\
\hline Panel A: & cointegration & & & & & & & & & \\
\hline Austria & Belgium & 1995 & 1998 & -0.61 & -0.84 & 1.03 & 10.80 & 0.30 & -1.44 & 0.42 \\
\hline Austria & Belgium & 1996 & 1999 & -5.13 & -3.04 & 1.64 & 6.88 & 0.16 & -1.27 & \\
\hline Austria & Belgium & 1997 & 2000 & 2.56 & 3.99 & 0.52 & 5.86 & 0.09 & -2.23 & \\
\hline Austria & Belgium & 1998 & 2001 & 2.68 & 7.25 & 0.47 & 9.69 & 0.15 & -1.21 & \\
\hline Austria & Belgium & 1999 & 2002 & 1.97 & 5.43 & 0.53 & 11.41 & 0.20 & -1.45 & \\
\hline Austria & Finland & 1995 & 1998 & 2.73 & 16.97 & 0.72 & 27.86 & 0.22 & -1.03 & \\
\hline Austria & Finland & 1996 & 1999 & 1.28 & 4.03 & 0.97 & 16.40 & 0.19 & -1.77 & \\
\hline Austria & Finland & 1997 & 2000 & 1.56 & 3.76 & 0.90 & 11.46 & 0.18 & -2.14 & \\
\hline Austria & Finland & 1998 & 2001 & 2.06 & 8.87 & 0.78 & 18.12 & 0.33 & -2.32 & \\
\hline Austria & Finland & 1999 & 2002 & 2.04 & 13.83 & 0.77 & 27.53 & 0.55 & -2.71 & \\
\hline Austria & France & 1995 & 1998 & 4.16 & 15.77 & 0.45 & 11.57 & 0.16 & -3.08 & \\
\hline Austria & France & 1996 & 1999 & 4.20 & 26.54 & 0.41 & 14.83 & 0.20 & -1.58 & \\
\hline Austria & France & 1997 & 2000 & 3.58 & 14.42 & 0.53 & 11.02 & 0.27 & -1.82 & -1.97 \\
\hline Austria & France & 1998 & 2001 & 3.21 & 11.54 & 0.60 & 10.99 & 0.30 & -1.82 & \\
\hline Austria & France & 1999 & 2002 & 2.62 & 14.73 & 0.69 & 19.61 & 0.89 & -4.04 & \\
\hline Austria & Germany & 1995 & 1998 & -1.81 & -6.17 & 0.92 & 30.62 & 0.47 & -2.32 & \\
\hline Austria & Germany & 1996 & 1999 & -3.18 & -3.80 & 1.05 & 11.54 & 0.19 & -1.25 & \\
\hline Austria & Germany & 1997 & 2000 & -1.17 & -1.01 & 0.82 & 6.45 & 0.08 & -1.50 & \\
\hline Austria & Germany & 1998 & 2001 & 0.60 & 0.77 & 0.60 & 7.31 & 0.10 & -0.79 & -1.25 \\
\hline Austria & Germany & 1999 & 2002 & -0.46 & -0.53 & 0.69 & 7.67 & 0.10 & -0.64 & \\
\hline Austria & Ireland & 1995 & 1998 & 1.09 & 0.51 & 0.60 & 2.82 & 0.04 & -1.99 & \\
\hline Austria & Ireland & 1996 & 1999 & 1.86 & 2.32 & 0.49 & 5.77 & 0.06 & -2.17 & \\
\hline Austria & Ireland & 1997 & 2000 & 2.02 & 4.94 & 0.45 & 10.50 & 0.20 & -1.69 & \\
\hline Austria & Ireland & 1998 & 2001 & 1.65 & 4.02 & 0.49 & 11.25 & 0.22 & -1.62 & -2.04 \\
\hline Austria & Ireland & 1999 & 2002 & -0.48 & -1.16 & 0.73 & 15.87 & 0.25 & -2.12 & \\
\hline Austria & Italy & 1995 & 1998 & 3.75 & 10.87 & 0.32 & 10.03 & 0.05 & -3.66 & -2.58 \\
\hline Austria & Italy & 1996 & 1999 & 4.37 & 59.07 & 0.24 & 29.58 & 0.36 & -2.39 & -2.81 \\
\hline Austria & Italy & 1997 & 2000 & 4.83 & 23.86 & 0.20 & 7.44 & 0.09 & 0.51 & -0.41 \\
\hline Austria & Italy & 1998 & 2001 & 4.18 & 12.30 & 0.31 & 6.12 & 0.08 & -1.23 & -1.28 \\
\hline Austria & Italy & 1999 & 2002 & 0.74 & 2.60 & 0.89 & 18.77 & 0.27 & -2.22 & \\
\hline Austria & Netherlands & 1995 & 1998 & 4.14 & 7.27 & 0.82 & 5.34 & 0.03 & 0.60 & \\
\hline Austria & Netherlands & 1996 & 1999 & 8.75 & 9.41 & -0.66 & -2.45 & 0.07 & -2.00 & \\
\hline Austria & Netherlands & 1997 & 2000 & 5.26 & 14.58 & 0.27 & 2.92 & 0.05 & -2.12 & -1.52 \\
\hline Austria & Netherlands & 1998 & 2001 & 4.33 & 19.90 & 0.45 & 8.92 & 0.12 & -1.72 & -2.05 \\
\hline Austria & Netherlands & 1999 & 2002 & 3.54 & 34.75 & 0.59 & 25.36 & 0.71 & -3.71 & \\
\hline Austria & Portugal & 1995 & 1998 & 4.25 & 28.62 & 0.28 & 20.09 & 0.34 & -2.07 & -1.58 \\
\hline Austria & Portugal & 1996 & 1999 & 4.56 & 58.31 & 0.23 & 25.63 & 0.61 & -2.96 & -2.77 \\
\hline Austria & Portugal & 1997 & 2000 & 4.87 & 25.25 & 0.21 & 7.57 & 0.14 & -0.09 & -0.47 \\
\hline Austria & Portugal & 1998 & 2001 & 4.16 & 12.74 & 0.34 & 6.44 & 0.13 & -1.62 & -1.39 \\
\hline Austria & Portugal & 1999 & 2002 & 1.84 & 6.74 & 0.77 & 15.61 & 0.91 & -4.13 & -3.80 \\
\hline Austria & Spain & 1995 & 1998 & 4.54 & 28.40 & 0.35 & 16.90 & 0.16 & -2.38 & -2.80 \\
\hline Austria & Spain & 1996 & 1999 & 4.59 & 43.88 & 0.32 & 18.93 & 0.30 & -1.83 & \\
\hline Austria & Spain & 1997 & 2000 & 3.83 & 22.85 & 0.48 & 14.92 & 0.57 & -2.72 & \\
\hline Austria & Spain & 1998 & 2001 & 3.20 & 19.89 & 0.62 & 19.07 & 1.34 & -4.77 & -4.70 \\
\hline Austria & Spain & 1999 & 2002 & 3.04 & 17.67 & 0.64 & 17.87 & 1.20 & -4.76 & \\
\hline Austria & Australia & 1995 & 1998 & 1.17 & 2.12 & 0.60 & 10.87 & 0.08 & -1.80 & -2.00 \\
\hline Austria & Australia & 1996 & 1999 & 1.72 & 3.99 & 0.51 & 11.13 & 0.12 & -1.02 & \\
\hline Austria & Australia & 1997 & 2000 & 1.08 & 1.42 & 0.57 & 6.84 & 0.11 & -1.28 & -1.50 \\
\hline Austria & Australia & 1998 & 2001 & 1.22 & 1.59 & 0.56 & 6.58 & 0.12 & -1.54 & -2.02 \\
\hline Austria & Australia & 1999 & 2002 & 0.05 & 0.08 & 0.67 & 9.86 & 0.19 & -1.30 & \\
\hline Austria & Canada & 1995 & 1998 & 4.65 & 13.66 & 0.38 & 7.50 & 0.06 & 0.40 & \\
\hline Austria & Canada & 1996 & 1999 & 7.39 & 11.80 & -0.15 & -1.46 & 0.04 & -2.26 & \\
\hline Austria & Canada & 1997 & 2000 & 6.77 & 14.81 & -0.08 & -1.06 & 0.06 & -0.41 & -1.31 \\
\hline Austria & Canada & 1998 & 2001 & 4.78 & 9.89 & 0.22 & 3.04 & 0.07 & -1.16 & -1.89 \\
\hline Austria & Canada & 1999 & 2002 & 4.90 & 17.98 & 0.20 & 4.44 & 0.07 & -0.92 & -2.26 \\
\hline Austria & Japan & 1995 & 1998 & 4.52 & 13.64 & 1.48 & 8.13 & 0.07 & -0.70 & -1.37 \\
\hline Austria & Japan & 1996 & 1999 & -1.63 & -2.33 & 5.20 & 11.64 & 0.12 & -2.73 & \\
\hline Austria & Japan & 1997 & 2000 & 2.00 & 2.99 & 2.85 & 6.44 & 0.08 & -0.14 & \\
\hline Austria & Japan & 1998 & 2001 & 3.07 & 3.44 & 2.18 & 3.57 & 0.08 & -0.83 & -1.57 \\
\hline Austria & Japan & 1999 & 2002 & -1.80 & -1.12 & 5.63 & 4.90 & 0.15 & -1.76 & -2.16 \\
\hline Austria & Switzerland & 1995 & 1998 & 0.87 & 2.97 & 1.32 & 21.43 & 0.24 & -2.15 & \\
\hline Austria & Switzerland & 1996 & 1999 & 0.94 & 2.58 & 1.27 & 15.24 & 0.23 & -1.58 & \\
\hline Austria & Switzerland & 1997 & 2000 & -0.10 & -0.18 & 1.53 & 11.04 & 0.30 & -2.07 & \\
\hline Austria & Switzerland & 1998 & 2001 & -1.56 & -2.35 & 1.88 & 11.82 & 0.38 & -2.06 & \\
\hline Austria & Switzerland & 1999 & 2002 & -1.49 & -3.22 & 1.85 & 16.37 & 0.51 & -1.84 & \\
\hline Austria & United Kingdom & 1995 & 1998 & 9.65 & 6.41 & -0.33 & -1.67 & 0.02 & -0.84 & -1.13 \\
\hline Austria & United Kingdom & 1996 & 1999 & 4.71 & 6.00 & 0.24 & 2.26 & 0.02 & -2.13 & \\
\hline Austria & United Kingdom & 1997 & 2000 & 3.81 & 7.43 & 0.34 & 4.86 & 0.07 & -0.88 & \\
\hline Austria & United Kingdom & 1998 & 2001 & 4.77 & 10.63 & 0.21 & 3.32 & 0.06 & -0.84 & \\
\hline Austria & United Kingdom & 1999 & 2002 & 4.05 & 9.29 & 0.33 & 4.71 & 0.07 & -1.13 & \\
\hline Austria & USA & 1995 & 1998 & -8.72 & -3.35 & 1.87 & 6.10 & 0.14 & -2.45 & -1.20 \\
\hline Austria & USA & 1996 & 1999 & -2.05 & -0.82 & 1.03 & 3.40 & 0.05 & -0.69 & \\
\hline Austria & USA & 1997 & 2000 & 1.95 & 2.22 & 0.51 & 4.97 & 0.07 & -2.09 & \\
\hline Austria & USA & 1998 & 2001 & 5.05 & 10.94 & 0.15 & 2.60 & 0.06 & -0.92 & -1.73 \\
\hline Austria & USA & 1999 & 2002 & 5.18 & 21.12 & 0.13 & 3.80 & 0.07 & -0.89 & -2.55 \\
\hline Belgium & Austria & 1995 & 1998 & 2.54 & 5.51 & 0.69 & 10.80 & 0.40 & -2.16 & -0.36 \\
\hline Belgium & Austria & 1996 & 1999 & 5.06 & 17.34 & 0.31 & 6.88 & 0.33 & -2.04 & \\
\hline Belgium & Austria & 1997 & 2000 & 2.04 & 2.28 & 0.83 & 5.86 & 0.11 & -0.80 & \\
\hline Belgium & Austria & 1998 & 2001 & -1.34 & -1.45 & 1.43 & 9.69 & 0.13 & -1.27 & -1.08 \\
\hline Belgium & Austria & 1999 & 2002 & -0.71 & -0.96 & 1.39 & 11.41 & 0.19 & -1.73 & -1.28 \\
\hline Belgium & Finland & 1995 & 1998 & 4.26 & 15.52 & 0.53 & 11.98 & 0.46 & -2.59 & -5.66 \\
\hline Belgium & Finland & 1996 & 1999 & 5.26 & 21.64 & 0.34 & 7.46 & 0.36 & -2.13 & \\
\hline Belgium & Finland & 1997 & 2000 & 0.93 & 2.14 & 1.21 & 14.58 & 0.36 & -2.36 & -1.29 \\
\hline Belgium & Finland & 1998 & 2001 & 0.29 & 0.72 & 1.36 & 18.14 & 0.34 & -1.71 & -1.26 \\
\hline Belgium & Finland & 1999 & 2002 & 1.82 & 3.84 & 1.13 & 12.49 & 0.17 & -1.61 & -1.07 \\
\hline
\end{tabular}


Table A7: Rolling cointegration of short-term corporate lending rates

\begin{tabular}{|c|c|c|c|c|c|c|c|c|c|c|}
\hline & national interest rate $\mathrm{r}$ & sample & eriod & & coin & gration r & egression: & $=\mathrm{a}+\mathrm{b}$ & & \\
\hline $\mathrm{r}_{1}$ & $\mathrm{r}_{2}$ & start & end & $\mathrm{a}$ & t-stat(a) & $\mathrm{b}$ & t-stat(b) & DW & $\mathrm{DF}$ & $\mathrm{ADF}$ \\
\hline Belgium & France & 1995 & 1998 & 6.06 & 16.45 & 0.22 & 4.00 & 0.22 & -2.39 & -5.64 \\
\hline Belgium & France & 1996 & 1999 & 6.60 & 44.28 & 0.08 & 3.20 & 0.25 & -1.97 & -2.14 \\
\hline Belgium & France & 1997 & 2000 & 5.21 & 10.05 & 0.40 & 4.01 & 0.13 & -0.79 & -1.21 \\
\hline Belgium & France & 1998 & 2001 & 1.91 & 5.09 & 1.13 & 15.34 & 0.79 & -3.43 & \\
\hline Belgium & France & 1999 & 2002 & 2.57 & 5.99 & 1.03 & 12.14 & 0.55 & -2.66 & -1.99 \\
\hline Belgium & Germany & 1995 & 1998 & 1.35 & 2.11 & 0.63 & 9.66 & 0.34 & -2.11 & -1.45 \\
\hline Belgium & Germany & 1996 & 1999 & 4.64 & 7.46 & 0.26 & 3.91 & 0.26 & -1.88 & \\
\hline Belgium & Germany & 1997 & 2000 & -5.65 & -8.08 & 1.41 & 18.49 & 0.70 & -3.60 & -3.34 \\
\hline Belgium & Germany & 1998 & 2001 & -5.44 & -10.91 & 1.39 & 26.22 & 0.85 & -3.48 & -3.49 \\
\hline Belgium & Germany & 1999 & 2002 & -5.74 & -9.43 & 1.41 & 22.14 & 0.67 & -2.91 & -2.39 \\
\hline Belgium & Ireland & 1995 & 1998 & 1.41 & 0.83 & 0.61 & 3.62 & 0.17 & -2.12 & \\
\hline Belgium & Ireland & 1996 & 1999 & 5.00 & 14.70 & 0.22 & 6.07 & 0.36 & -2.63 & -2.68 \\
\hline Belgium & Ireland & 1997 & 2000 & 4.84 & 5.46 & 0.26 & 2.74 & 0.09 & 0.50 & -0.50 \\
\hline Belgium & Ireland & 1998 & 2001 & 2.11 & 1.88 & 0.59 & 4.90 & 0.08 & -1.48 & -1.70 \\
\hline Belgium & Ireland & 1999 & 2002 & -3.47 & -8.51 & 1.24 & 27.56 & 0.94 & -3.70 & -1.31 \\
\hline Belgium & Italy & 1995 & 1998 & 5.99 & 13.30 & 0.14 & 3.40 & 0.15 & -2.21 & -5.82 \\
\hline Belgium & Italy & 1996 & 1999 & 6.52 & 55.97 & 0.06 & 4.89 & 0.29 & -2.00 & -2.21 \\
\hline Belgium & Italy & 1997 & 2000 & 7.24 & 19.00 & 0.00 & 0.06 & 0.08 & 0.82 & -0.71 \\
\hline Belgium & Italy & 1998 & 2001 & 6.29 & 8.09 & 0.20 & 1.72 & 0.05 & -1.08 & -1.43 \\
\hline Belgium & Italy & 1999 & 2002 & -0.49 & -0.80 & 1.36 & 13.67 & 0.28 & -3.30 & \\
\hline Belgium & Netherlands & 1995 & 1998 & 4.01 & 14.03 & 0.95 & 12.34 & 0.62 & -2.82 & \\
\hline Belgium & Netherlands & 1996 & 1999 & 6.06 & 15.09 & 0.29 & 2.50 & 0.14 & -1.81 & -1.95 \\
\hline Belgium & Netherlands & 1997 & 2000 & 4.15 & 23.48 & 0.81 & 17.88 & 0.43 & -2.93 & -2.57 \\
\hline Belgium & Netherlands & 1998 & 2001 & 3.54 & 23.27 & 0.96 & 27.27 & 0.64 & -2.62 & -0.52 \\
\hline Belgium & Netherlands & 1999 & 2002 & 3.75 & 16.77 & 0.93 & 18.08 & 0.37 & -1.75 & -0.27 \\
\hline Belgium & Portugal & 1995 & 1998 & 5.82 & 20.60 & 0.16 & 6.10 & 0.27 & -1.92 & -4.47 \\
\hline Belgium & Portugal & 1996 & 1999 & 6.59 & 59.53 & 0.06 & 4.46 & 0.29 & -2.03 & -2.16 \\
\hline Belgium & Portugal & 1997 & 2000 & 7.20 & 19.63 & 0.01 & 0.16 & 0.08 & 0.83 & -0.68 \\
\hline Belgium & Portugal & 1998 & 2001 & 6.33 & 8.27 & 0.21 & 1.70 & 0.05 & -1.07 & -1.21 \\
\hline Belgium & Portugal & 1999 & 2002 & 2.29 & 3.02 & 0.98 & 7.21 & 0.21 & -2.18 & \\
\hline Belgium & Spain & 1995 & 1998 & 6.02 & 22.34 & 0.20 & 5.67 & 0.19 & -2.01 & -5.17 \\
\hline Belgium & Spain & 1996 & 1999 & 6.59 & 58.26 & 0.08 & 4.32 & 0.28 & -1.90 & -2.18 \\
\hline Belgium & Spain & 1997 & 2000 & 5.84 & 12.48 & 0.28 & 3.09 & 0.09 & 0.02 & -0.22 \\
\hline Belgium & Spain & 1998 & 2001 & 3.00 & 6.18 & 0.94 & 9.58 & 0.32 & -2.23 & -1.63 \\
\hline Belgium & Spain & 1999 & 2002 & 3.00 & 8.79 & 1.00 & 14.01 & 0.69 & -3.05 & -1.29 \\
\hline Belgium & Australia & 1995 & 1998 & 4.56 & 6.20 & 0.29 & 4.02 & 0.17 & -1.98 & -5.42 \\
\hline Belgium & Australia & 1996 & 1999 & 6.16 & 18.53 & 0.10 & 2.74 & 0.22 & -1.86 & -2.23 \\
\hline Belgium & Australia & 1997 & 2000 & 0.49 & 0.52 & 0.75 & 7.20 & 0.15 & -1.44 & -2.12 \\
\hline Belgium & Australia & 1998 & 2001 & -0.85 & -0.61 & 0.94 & 6.13 & 0.09 & -0.72 & \\
\hline Belgium & Australia & 1999 & 2002 & 0.73 & 0.52 & 0.78 & 4.98 & 0.07 & -0.99 & \\
\hline Belgium & Canada & 1995 & 1998 & 5.02 & 27.51 & 0.37 & 13.88 & 0.60 & -2.90 & \\
\hline Belgium & Canada & 1996 & 1999 & 6.65 & 24.58 & 0.07 & 1.53 & 0.19 & -1.85 & -1.98 \\
\hline Belgium & Canada & 1997 & 2000 & 5.11 & 10.38 & 0.34 & 4.43 & 0.10 & 0.07 & -0.60 \\
\hline Belgium & Canada & 1998 & 2001 & 6.83 & 7.42 & 0.12 & 0.86 & 0.05 & -0.65 & -1.34 \\
\hline Belgium & Canada & 1999 & 2002 & 7.41 & 14.15 & 0.05 & 0.62 & 0.05 & -1.08 & -2.32 \\
\hline Belgium & Japan & 1995 & 1998 & 4.98 & 26.68 & 1.41 & 13.80 & 0.58 & -3.08 & -2.13 \\
\hline Belgium & Japan & 1996 & 1999 & 4.00 & 10.12 & 1.97 & 7.78 & 0.41 & -2.55 & \\
\hline Belgium & Japan & 1997 & 2000 & 7.00 & 5.98 & 0.18 & 0.23 & 0.08 & 0.83 & -0.68 \\
\hline Belgium & Japan & 1998 & 2001 & 8.03 & 4.56 & -0.29 & -0.24 & 0.05 & -0.82 & -1.64 \\
\hline Belgium & Japan & 1999 & 2002 & -0.02 & -0.01 & 5.53 & 2.59 & 0.08 & -1.82 & \\
\hline Belgium & Switzerland & 1995 & 1998 & 3.72 & 6.52 & 0.80 & 6.68 & 0.24 & -1.99 & -4.40 \\
\hline Belgium & Switzerland & 1996 & 1999 & 5.88 & 16.92 & 0.27 & 3.42 & 0.24 & -1.93 & -2.14 \\
\hline Belgium & Switzerland & 1997 & 2000 & 2.74 & 2.21 & 1.08 & 3.66 & 0.10 & -0.56 & -0.74 \\
\hline Belgium & Switzerland & 1998 & 2001 & -6.99 & -8.08 & 3.53 & 16.90 & 0.68 & -4.08 & \\
\hline Belgium & Switzerland & 1999 & 2002 & -2.92 & -2.52 & 2.60 & 9.19 & 0.22 & -0.73 & \\
\hline Belgium & United Kingdom & 1995 & 1998 & 6.31 & 5.03 & 0.16 & 0.94 & 0.12 & -2.25 & -6.24 \\
\hline Belgium & United Kingdom & 1996 & 1999 & 5.34 & 21.13 & 0.24 & 6.84 & 0.37 & -3.06 & \\
\hline Belgium & United Kingdom & 1997 & 2000 & 6.19 & 7.88 & 0.15 & 1.37 & 0.08 & 1.04 & -0.25 \\
\hline Belgium & United Kingdom & 1998 & 2001 & 8.28 & 9.54 & -0.10 & -0.78 & 0.06 & -0.84 & -1.77 \\
\hline Belgium & United Kingdom & 1999 & 2002 & 7.09 & 8.32 & 0.11 & 0.76 & 0.05 & -1.14 & -2.24 \\
\hline Belgium & USA & 1995 & 1998 & -6.71 & -3.44 & 1.68 & 7.28 & 0.44 & -2.99 & -1.07 \\
\hline Belgium & USA & 1996 & 1999 & 3.28 & 3.04 & 0.46 & 3.50 & 0.25 & -2.00 & -2.55 \\
\hline Belgium & USA & 1997 & 2000 & -0.71 & -1.00 & 0.94 & 11.17 & 0.30 & -1.49 & -2.08 \\
\hline Belgium & USA & 1998 & 2001 & 7.52 & 8.69 & 0.01 & 0.10 & 0.05 & -0.81 & -1.59 \\
\hline Belgium & USA & 1999 & 2002 & 7.65 & 16.87 & 0.01 & 0.17 & 0.05 & -1.06 & -2.31 \\
\hline Finland & Austria & 1995 & 1998 & -3.23 & -9.56 & 1.31 & 27.86 & 0.22 & -1.08 & \\
\hline Finland & Austria & 1996 & 1999 & -0.35 & -0.99 & 0.88 & 16.40 & 0.19 & -2.43 & \\
\hline Finland & Austria & 1997 & 2000 & 0.08 & 0.18 & 0.82 & 11.46 & 0.18 & -1.28 & \\
\hline Finland & Austria & 1998 & 2001 & -1.66 & -4.26 & 1.12 & 18.12 & 0.32 & -2.27 & \\
\hline Finland & Austria & 1999 & 2002 & -2.19 & -8.11 & 1.22 & 27.53 & 0.53 & -2.64 & \\
\hline Finland & Belgium & 1995 & 1998 & -4.62 & -5.13 & 1.43 & 11.98 & 0.35 & -1.98 & -3.02 \\
\hline Finland & Belgium & 1996 & 1999 & -6.16 & -3.98 & 1.63 & 7.46 & 0.19 & -2.05 & \\
\hline Finland & Belgium & 1997 & 2000 & 0.30 & 0.88 & 0.68 & 14.58 & 0.33 & -2.47 & -2.28 \\
\hline Finland & Belgium & 1998 & 2001 & 0.47 & 1.74 & 0.64 & 18.14 & 0.34 & -1.63 & -1.37 \\
\hline Finland & Belgium & 1999 & 2002 & -0.06 & -0.13 & 0.68 & 12.49 & 0.16 & -1.17 & -1.27 \\
\hline Finland & France & 1995 & 1998 & 2.36 & 5.70 & 0.57 & 9.33 & 0.10 & -2.20 & \\
\hline Finland & France & 1996 & 1999 & 3.34 & 17.31 & 0.36 & 10.82 & 0.14 & -2.43 & -2.32 \\
\hline Finland & France & 1997 & 2000 & 3.23 & 9.55 & 0.39 & 5.99 & 0.14 & -0.93 & -2.08 \\
\hline Finland & France & 1998 & 2001 & 1.55 & 5.37 & 0.76 & 13.30 & 0.50 & -2.45 & -2.54 \\
\hline Finland & France & 1999 & 2002 & 0.98 & 3.73 & 0.85 & 16.36 & 0.70 & -3.40 & \\
\hline Finland & Germany & 1995 & 1998 & -5.89 & -13.53 & 1.23 & 27.72 & 0.37 & -2.11 & \\
\hline Finland & Germany & 1996 & 1999 & -4.56 & -7.68 & 1.07 & 16.76 & 0.33 & -2.05 & \\
\hline Finland & Germany & 1997 & 2000 & -3.88 & -5.44 & 1.00 & 12.80 & 0.13 & -1.17 & \\
\hline Finland & Germany & 1998 & 2001 & -2.77 & -4.27 & 0.87 & 12.54 & 0.12 & -0.41 & -0.91 \\
\hline Finland & Germany & 1999 & 2002 & -3.19 & -3.07 & 0.88 & 8.11 & 0.05 & -0.04 & \\
\hline Finland & Ireland & 1995 & 8 & -1.30 & -0.44 & 74 & 2.52 & 0.04 & -1.68 & -1.68 \\
\hline
\end{tabular}


Table A7: Rolling cointegration of short-term corporate lending rates

\begin{tabular}{|c|c|c|c|c|c|c|c|c|c|c|}
\hline & national interest rate $r$ & sample & eriod & & coin & gration & egression: & $=\mathrm{a}+\mathrm{b}$ & & \\
\hline $\mathrm{r}_{1}$ & $\mathrm{r}_{2}$ & start & end & $\mathrm{a}$ & t-stat(a) & $\mathrm{b}$ & t-stat(b) & DW & DF & $\mathrm{ADF}$ \\
\hline Finland & Ireland & 1996 & 1999 & 2.32 & 2.57 & 0.32 & 3.41 & 0.05 & -3.30 & -2.68 \\
\hline Finland & Ireland & 1997 & 2000 & 2.17 & 3.91 & 0.33 & 5.53 & 0.11 & 0.17 & -0.90 \\
\hline Finland & Ireland & 1998 & 2001 & 0.64 & 0.99 & 0.51 & 7.32 & 0.13 & -1.56 & -1.96 \\
\hline Finland & Ireland & 1999 & 2002 & -3.21 & -7.12 & 0.94 & 18.77 & 0.19 & -2.08 & -1.53 \\
\hline Finland & Italy & 1995 & 1998 & 1.85 & 3.52 & 0.41 & 8.28 & 0.03 & -2.27 & -1.84 \\
\hline Finland & Italy & 1996 & 1999 & 3.53 & 24.82 & 0.21 & 13.50 & 0.09 & -2.77 & -2.18 \\
\hline Finland & Italy & 1997 & 2000 & 4.55 & 17.09 & 0.09 & 2.66 & 0.05 & 1.32 & -2.57 \\
\hline Finland & Italy & 1998 & 2001 & 3.59 & 7.44 & 0.27 & 3.72 & 0.04 & -1.17 & -2.39 \\
\hline Finland & Italy & 1999 & 2002 & -1.73 & -7.42 & 1.15 & 29.94 & 0.27 & -2.72 & \\
\hline Finland & Netherlands & 1995 & 1998 & 1.64 & 2.30 & 1.22 & 6.41 & 0.03 & 0.00 & -0.54 \\
\hline Finland & Netherlands & 1996 & 1999 & 6.94 & 7.57 & -0.46 & -1.71 & 0.05 & -3.28 & -2.45 \\
\hline Finland & Netherlands & 1997 & 2000 & 3.37 & 13.57 & 0.49 & 7.62 & 0.08 & -1.90 & \\
\hline Finland & Netherlands & 1998 & 2001 & 2.72 & 15.75 & 0.62 & 15.53 & 0.23 & -2.02 & -2.14 \\
\hline Finland & Netherlands & 1999 & 2002 & 2.02 & 16.48 & 0.75 & 26.51 & 0.71 & -3.80 & \\
\hline Finland & Portugal & 1995 & 1998 & 2.47 & 8.61 & 0.35 & 13.18 & 0.16 & -1.01 & -1.43 \\
\hline Finland & Portugal & 1996 & 1999 & 3.69 & 27.50 & 0.20 & 13.13 & 0.18 & -2.44 & -1.77 \\
\hline Finland & Portugal & 1997 & 2000 & 4.56 & 17.84 & 0.10 & 2.72 & 0.06 & 1.06 & -1.77 \\
\hline Finland & Portugal & 1998 & 2001 & 3.61 & 7.62 & 0.29 & 3.75 & 0.06 & -1.32 & -1.68 \\
\hline Finland & Portugal & 1999 & 2002 & -0.16 & -0.48 & 0.97 & 16.67 & 0.95 & -4.30 & \\
\hline Finland & Spain & 1995 & 1998 & 2.69 & 11.19 & 0.46 & 14.90 & 0.10 & -1.45 & \\
\hline Finland & Spain & 1996 & 1999 & 3.59 & 32.76 & 0.30 & 17.04 & 0.23 & -1.89 & \\
\hline Finland & Spain & 1997 & 2000 & 3.51 & 12.27 & 0.34 & 6.14 & 0.12 & -0.46 & -0.80 \\
\hline Finland & Spain & 1998 & 2001 & 1.82 & 7.81 & 0.72 & 15.38 & 0.74 & -3.46 & \\
\hline Finland & Spain & 1999 & 2002 & 1.41 & 6.49 & 0.81 & 17.84 & 0.98 & -4.14 & -2.45 \\
\hline Finland & Australia & 1995 & 1998 & -1.48 & -1.75 & 0.76 & 9.08 & 0.06 & -1.26 & -1.52 \\
\hline Finland & Australia & 1996 & 1999 & 0.84 & 2.02 & 0.48 & 11.00 & 0.12 & -1.99 & -1.91 \\
\hline Finland & Australia & 1997 & 2000 & 0.24 & 0.33 & 0.55 & 6.96 & 0.13 & -0.99 & -2.58 \\
\hline Finland & Australia & 1998 & 2001 & -0.69 & -0.75 & 0.67 & 6.62 & 0.10 & -1.14 & -1.77 \\
\hline Finland & Australia & 1999 & 2002 & -1.50 & -1.61 & 0.75 & 7.26 & 0.10 & -0.76 & -1.43 \\
\hline Finland & Canada & 1995 & 1998 & 2.36 & 6.22 & 0.57 & 10.22 & 0.09 & -0.36 & -0.72 \\
\hline Finland & Canada & 1996 & 1999 & 4.92 & 8.09 & 0.08 & 0.76 & 0.03 & -3.02 & -1.68 \\
\hline Finland & Canada & 1997 & 2000 & 4.39 & 10.40 & 0.13 & 2.02 & 0.06 & 0.14 & -2.18 \\
\hline Finland & Canada & 1998 & 2001 & 4.03 & 6.66 & 0.20 & 2.23 & 0.05 & -0.73 & -2.01 \\
\hline Finland & Canada & 1999 & 2002 & 3.88 & 10.93 & 0.23 & 3.88 & 0.03 & -0.57 & -2.72 \\
\hline Finland & Japan & 1995 & 1998 & 2.83 & 5.81 & 1.86 & 6.96 & 0.07 & -1.01 & \\
\hline Finland & Japan & 1996 & 1999 & -0.94 & -1.00 & 4.05 & 6.72 & 0.08 & -3.70 & -2.94 \\
\hline Finland & Japan & 1997 & 2000 & 2.95 & 3.64 & 1.52 & 2.84 & 0.06 & 1.08 & -0.85 \\
\hline Finland & Japan & 1998 & 2001 & 3.38 & 2.88 & 1.36 & 1.69 & 0.05 & -0.72 & -2.39 \\
\hline Finland & Japan & 1999 & 2002 & -4.48 & -2.19 & 6.94 & 4.75 & 0.13 & -1.58 & -2.30 \\
\hline Finland & Switzerland & 1995 & 1998 & -1.89 & -3.37 & 1.69 & 14.39 & 0.09 & -1.05 & -1.91 \\
\hline Finland & Switzerland & 1996 & 1999 & 0.45 & 1.00 & 1.13 & 10.92 & 0.12 & -2.35 & -2.32 \\
\hline Finland & Switzerland & 1997 & 2000 & 0.47 & 0.59 & 1.14 & 6.08 & 0.09 & -0.78 & -1.30 \\
\hline Finland & Switzerland & 1998 & 2001 & -4.76 & -8.56 & 2.45 & 18.24 & 0.58 & -3.20 & \\
\hline Finland & Switzerland & 1999 & 2002 & -4.50 & -8.94 & 2.37 & 19.36 & 0.54 & -1.79 & -2.10 \\
\hline Finland & United Kingdom & 1995 & 1998 & 8.00 & 3.86 & -0.25 & -0.90 & 0.01 & -1.43 & -1.58 \\
\hline Finland & United Kingdom & 1996 & 1999 & 4.12 & 5.35 & 0.17 & 1.64 & 0.03 & -3.98 & -2.12 \\
\hline Finland & United Kingdom & 1997 & 2000 & 3.47 & 6.41 & 0.24 & 3.28 & 0.06 & 1.24 & -2.03 \\
\hline Finland & United Kingdom & 1998 & 2001 & 4.65 & 7.88 & 0.10 & 1.22 & 0.04 & -0.69 & -2.92 \\
\hline Finland & United Kingdom & 1999 & 2002 & 2.89 & 5.08 & 0.38 & 4.14 & 0.04 & -0.85 & -2.18 \\
\hline Finland & USA & 1995 & 1998 & -14.69 & -4.10 & 2.46 & 5.81 & 0.14 & -1.96 & -1.01 \\
\hline Finland & USA & 1996 & 1999 & 0.72 & 0.28 & 0.56 & 1.80 & 0.04 & -2.44 & -2.25 \\
\hline Finland & USA & 1997 & 2000 & 0.20 & 0.28 & 0.59 & 7.03 & 0.13 & -1.10 & -1.89 \\
\hline Finland & USA & 1998 & 2001 & 4.57 & 7.85 & 0.10 & 1.37 & 0.04 & -0.71 & -2.67 \\
\hline Finland & USA & 1999 & 2002 & 4.21 & 13.27 & 0.14 & 3.33 & 0.04 & -0.61 & -2.80 \\
\hline France & Austria & 1995 & 1998 & -5.17 & -5.05 & 1.65 & 11.57 & 0.20 & -2.40 & \\
\hline France & Austria & 1996 & 1999 & -7.58 & -8.48 & 2.04 & 14.83 & 0.21 & -1.53 & -1.81 \\
\hline France & Austria & 1997 & 2000 & -3.48 & -4.47 & 1.36 & 11.02 & 0.32 & -2.38 & -2.50 \\
\hline France & Austria & 1998 & 2001 & -2.47 & -3.61 & 1.20 & 10.99 & 0.36 & -2.08 & \\
\hline France & Austria & 1999 & 2002 & -2.84 & -7.09 & 1.29 & 19.61 & 0.96 & -4.33 & \\
\hline France & Belgium & 1995 & 1998 & -2.27 & -1.02 & 1.19 & 4.00 & 0.15 & -1.04 & \\
\hline France & Belgium & 1996 & 1999 & -9.97 & -2.05 & 2.21 & 3.20 & 0.08 & -1.08 & -1.51 \\
\hline France & Belgium & 1997 & 2000 & 0.43 & 0.37 & 0.64 & 4.01 & 0.15 & -2.76 & -2.40 \\
\hline France & Belgium & 1998 & 2001 & -0.58 & -1.59 & 0.74 & 15.34 & 0.86 & -3.60 & \\
\hline France & Belgium & 1999 & 2002 & -0.70 & -1.49 & 0.74 & 12.14 & 0.62 & -2.82 & \\
\hline France & Finland & 1995 & 1998 & -0.42 & -0.55 & 1.15 & 9.33 & 0.14 & -1.45 & \\
\hline France & Finland & 1996 & 1999 & -5.05 & -5.09 & 1.98 & 10.82 & 0.14 & -1.59 & -1.98 \\
\hline France & Finland & 1997 & 2000 & -0.73 & -0.75 & 1.11 & 5.99 & 0.18 & -2.55 & -2.46 \\
\hline France & Finland & 1998 & 2001 & -0.59 & -1.38 & 1.05 & 13.30 & 0.58 & -2.70 & \\
\hline France & Finland & 1999 & 2002 & -0.25 & -0.77 & 1.00 & 16.36 & 0.79 & -3.77 & \\
\hline France & Germany & 1995 & 1998 & -8.59 & -6.65 & 1.55 & 11.81 & 0.27 & -2.53 & \\
\hline France & Germany & 1996 & 1999 & -17.28 & -11.38 & 2.48 & 15.10 & 0.39 & -2.43 & \\
\hline France & Germany & 1997 & 2000 & -6.28 & -3.25 & 1.24 & 5.89 & 0.16 & -2.42 & -2.25 \\
\hline France & Germany & 1998 & 2001 & -4.62 & -6.26 & 1.03 & 13.10 & 0.54 & -2.67 & -2.86 \\
\hline France & Germany & 1999 & 2002 & -4.28 & -3.88 & 0.97 & 8.42 & 0.29 & -1.61 & \\
\hline France & Ireland & 1995 & 1998 & 2.68 & 0.61 & 0.39 & 0.89 & 0.05 & -0.47 & \\
\hline France & Ireland & 1996 & 1999 & -0.31 & -0.14 & 0.62 & 2.71 & 0.04 & -1.48 & -2.01 \\
\hline France & Ireland & 1997 & 2000 & 0.44 & 0.45 & 0.49 & 4.72 & 0.16 & -2.10 & -2.04 \\
\hline France & Ireland & 1998 & 2001 & 0.45 & 0.50 & 0.49 & 5.16 & 0.20 & -1.72 & \\
\hline France & Ireland & 1999 & 2002 & -3.87 & -6.51 & 0.98 & 14.94 & 0.74 & -3.20 & \\
\hline France & Italy & 1995 & 1998 & -0.99 & -3.40 & 0.72 & 26.53 & 0.66 & -2.77 & \\
\hline France & Italy & 1996 & 1999 & 1.01 & 4.41 & 0.52 & 20.86 & 0.31 & -1.65 & -1.87 \\
\hline France & Italy & 1997 & 2000 & 3.05 & 8.29 & 0.28 & 5.70 & 0.15 & -0.95 & -1.19 \\
\hline France & Italy & 1998 & 2001 & 3.77 & 6.10 & 0.19 & 2.05 & 0.13 & -1.34 & -1.55 \\
\hline France & Italy & 1999 & 2002 & -1.83 & -3.38 & 1.13 & 12.64 & 0.55 & -3.66 & \\
\hline France & Netherlands & 1995 & 1998 & 4.93 & 3.62 & 0.46 & 1.24 & 0.05 & -0.08 & \\
\hline France & therlands & 1996 & 9 & 15.18 & 8.93 & -2.80 & -5.65 & 0.18 & -2.44 & \\
\hline
\end{tabular}


Table A7: Rolling cointegration of short-term corporate lending rates

\begin{tabular}{|c|c|c|c|c|c|c|c|c|c|c|}
\hline & national interest rate $\mathrm{r}$ & sample & eriod & & coil & egration & egression & $=\mathrm{a}+\mathrm{b}$ & & \\
\hline $\mathrm{r}_{1}$ & $\mathrm{r}_{2}$ & start & end & $\mathrm{a}$ & t-stat(a) & $\mathrm{b}$ & t-stat(b) & DW & DF & $\mathrm{ADF}$ \\
\hline France & Netherlands & 1997 & 2000 & 3.91 & 6.48 & 0.31 & 2.00 & 0.11 & -2.57 & -1.97 \\
\hline France & Netherlands & 1998 & 2001 & 1.91 & 9.60 & 0.73 & 15.87 & 1.04 & -4.24 & \\
\hline France & Netherlands & 1999 & 2002 & 1.63 & 8.69 & 0.78 & 18.22 & 1.47 & -5.19 & \\
\hline France & Portugal & 1995 & 1998 & 1.14 & 3.66 & 0.52 & 18.08 & 0.57 & -4.10 & \\
\hline France & Portugal & 1996 & 1999 & 1.35 & 6.99 & 0.51 & 22.97 & 0.59 & -2.63 & -2.55 \\
\hline France & Portugal & 1997 & 2000 & 2.98 & 8.92 & 0.31 & 6.49 & 0.20 & -0.99 & -1.03 \\
\hline France & Portugal & 1998 & 2001 & 3.66 & 6.08 & 0.22 & 2.29 & 0.13 & -1.39 & -1.53 \\
\hline France & Portugal & 1999 & 2002 & -0.31 & -0.62 & 0.96 & 10.56 & 0.73 & -4.05 & \\
\hline France & Spain & 1995 & 1998 & 1.50 & 5.95 & 0.68 & 21.01 & 0.64 & -3.36 & \\
\hline France & Spain & 1996 & 1999 & 1.28 & 6.73 & 0.73 & 23.74 & 0.55 & -3.34 & -3.34 \\
\hline France & Spain & 1997 & 2000 & 1.33 & 4.14 & 0.74 & 11.94 & 0.51 & -2.43 & -2.79 \\
\hline France & Spain & 1998 & 2001 & 1.27 & 3.32 & 0.77 & 9.92 & 0.54 & -2.78 & \\
\hline France & Spain & 1999 & 2002 & 0.87 & 3.56 & 0.87 & 17.14 & 1.44 & -5.09 & \\
\hline France & Australia & 1995 & 1998 & -6.27 & -10.90 & 1.28 & 22.52 & 0.67 & -3.50 & \\
\hline France & Australia & 1996 & 1999 & -6.50 & -14.43 & 1.29 & 27.06 & 0.68 & -3.09 & \\
\hline France & Australia & 1997 & 2000 & -4.97 & -5.57 & 1.11 & 11.30 & 0.49 & -2.96 & \\
\hline France & Australia & 1998 & 2001 & -2.49 & -2.45 & 0.84 & 7.43 & 0.35 & -2.03 & \\
\hline France & Australia & 1999 & 2002 & -2.39 & -2.41 & 0.82 & 7.46 & 0.35 & -2.04 & \\
\hline France & Canada & 1995 & 1998 & 3.90 & 4.43 & 0.41 & 3.15 & 0.06 & -0.13 & \\
\hline France & Canada & 1996 & 1999 & 8.47 & 6.18 & -0.47 & -2.10 & 0.04 & -2.17 & -2.06 \\
\hline France & Canada & 1997 & 2000 & 6.68 & 9.51 & -0.25 & -2.28 & 0.11 & -1.09 & -1.41 \\
\hline France & Canada & 1998 & 2001 & 3.88 & 5.33 & 0.18 & 1.59 & 0.12 & -1.13 & -1.42 \\
\hline France & Canada & 1999 & 2002 & 3.76 & 9.33 & 0.21 & 3.13 & 0.14 & -1.60 & \\
\hline France & Japan & 1995 & 1998 & 3.81 & 4.27 & 1.57 & 3.21 & 0.08 & -0.81 & \\
\hline France & Japan & 1996 & 1999 & -8.18 & -3.49 & 8.85 & 5.90 & 0.07 & -1.47 & \\
\hline France & Japan & 1997 & 2000 & 0.76 & 0.57 & 2.88 & 3.27 & 0.13 & -1.49 & -1.36 \\
\hline France & Japan & 1998 & 2001 & 4.98 & 3.51 & 0.03 & 0.03 & 0.12 & -1.20 & -1.60 \\
\hline France & Japan & 1999 & 2002 & -1.99 & -0.79 & 4.98 & 2.79 & 0.20 & -2.07 & \\
\hline France & Switzerland & 1995 & 1998 & -5.29 & -8.76 & 2.51 & 19.84 & 0.52 & -3.54 & \\
\hline France & Switzerland & 1996 & 1999 & -7.71 & -20.33 & 3.06 & 35.31 & 1.37 & -4.86 & \\
\hline France & Switzerland & 1997 & 2000 & -6.23 & -10.54 & 2.71 & 19.19 & 1.19 & -4.48 & \\
\hline France & Switzerland & 1998 & 2001 & -6.61 & -8.91 & 2.81 & 15.70 & 1.26 & -4.97 & \\
\hline France & Switzerland & 1999 & 2002 & -4.97 & -6.64 & 2.43 & 13.34 & 0.93 & -3.37 & \\
\hline France & United Kingdom & 1995 & 1998 & 18.13 & 7.49 & -1.51 & -4.77 & 0.12 & -0.18 & \\
\hline France & United Kingdom & 1996 & 1999 & 6.43 & 3.47 & -0.11 & -0.44 & 0.03 & -1.35 & -1.69 \\
\hline France & United Kingdom & 1997 & 2000 & 3.85 & 3.88 & 0.17 & 1.26 & 0.10 & -1.81 & -1.64 \\
\hline France & United Kingdom & 1998 & 2001 & 4.95 & 7.03 & 0.01 & 0.11 & 0.12 & -1.20 & -1.60 \\
\hline France & United Kingdom & 1999 & 2002 & 2.75 & 4.28 & 0.37 & 3.53 & 0.16 & -1.85 & \\
\hline France & USA & 1995 & 1998 & -11.53 & -1.88 & 2.14 & 2.96 & 0.09 & -1.02 & -1.27 \\
\hline France & USA & 1996 & 1999 & -4.90 & -0.81 & 1.27 & 1.73 & 0.05 & -1.06 & -1.71 \\
\hline France & USA & 1997 & 2000 & -1.58 & -1.10 & 0.78 & 4.67 & 0.18 & -3.01 & -2.57 \\
\hline France & USA & 1998 & 2001 & 4.10 & 6.00 & 0.11 & 1.37 & 0.12 & -1.16 & -1.50 \\
\hline France & USA & 1999 & 2002 & 4.05 & 11.40 & 0.13 & 2.75 & 0.14 & -1.53 & \\
\hline Germany & Austria & 1995 & 1998 & 2.34 & 9.59 & 1.04 & 30.62 & 0.48 & -2.41 & \\
\hline Germany & Austria & 1996 & 1999 & 4.64 & 11.56 & 0.71 & 11.54 & 0.21 & -1.81 & \\
\hline Germany & Austria & 1997 & 2000 & 5.49 & 9.64 & 0.58 & 6.45 & 0.07 & 0.90 & \\
\hline Germany & Austria & 1998 & 2001 & 3.80 & 4.98 & 0.89 & 7.31 & 0.06 & -0.64 & -0.66 \\
\hline Germany & Austria & 1999 & 2002 & 4.56 & 7.00 & 0.82 & 7.67 & 0.07 & -1.12 & \\
\hline Germany & Belgium & 1995 & 1998 & 1.79 & 2.16 & 1.07 & 9.66 & 0.24 & -1.47 & -0.01 \\
\hline Germany & Belgium & 1996 & 1999 & 2.53 & 1.47 & 0.95 & 3.91 & 0.11 & -1.74 & \\
\hline Germany & Belgium & 1997 & 2000 & 4.61 & 18.75 & 0.62 & 18.49 & 0.67 & -3.35 & -2.93 \\
\hline Germany & Belgium & 1998 & 2001 & 4.24 & 21.62 & 0.67 & 26.22 & 0.83 & -3.41 & -3.40 \\
\hline Germany & Belgium & 1999 & 2002 & 4.54 & 19.98 & 0.65 & 22.14 & 0.64 & -2.94 & -2.51 \\
\hline Germany & Finland & 1995 & 1998 & 5.07 & 29.45 & 0.77 & 27.72 & 0.38 & -2.18 & \\
\hline Germany & Finland & 1996 & 1999 & 4.94 & 19.17 & 0.80 & 16.76 & 0.34 & -1.99 & \\
\hline Germany & Finland & 1997 & 2000 & 5.04 & 15.68 & 0.78 & 12.80 & 0.12 & -0.39 & \\
\hline Germany & Finland & 1998 & 2001 & 4.59 & 11.99 & 0.89 & 12.54 & 0.10 & -0.33 & \\
\hline Germany & Finland & 1999 & 2002 & 6.05 & 13.98 & 0.67 & 8.11 & 0.04 & -0.90 & \\
\hline Germany & France & 1995 & 1998 & 6.58 & 23.75 & 0.48 & 11.81 & 0.24 & -3.18 & \\
\hline Germany & France & 1996 & 1999 & 7.36 & 57.24 & 0.34 & 15.10 & 0.41 & -2.91 & \\
\hline Germany & France & 1997 & 2000 & 7.38 & 24.44 & 0.35 & 5.89 & 0.11 & 0.23 & -0.15 \\
\hline Germany & France & 1998 & 2001 & 5.52 & 18.64 & 0.77 & 13.10 & 0.44 & -2.40 & -2.55 \\
\hline Germany & France & 1999 & 2002 & 6.42 & 17.21 & 0.62 & 8.42 & 0.19 & -1.41 & \\
\hline Germany & Ireland & 1995 & 1998 & 5.17 & 2.17 & 0.46 & 1.93 & 0.03 & -1.94 & \\
\hline Germany & Ireland & 1996 & 1999 & 7.61 & 9.11 & 0.17 & 1.96 & 0.05 & -2.51 & \\
\hline Germany & Ireland & 1997 & 2000 & 7.51 & 12.77 & 0.17 & 2.79 & 0.04 & 2.70 & -0.31 \\
\hline Germany & Ireland & 1998 & 2001 & 6.06 & 7.28 & 0.36 & 3.99 & 0.03 & -1.16 & -1.48 \\
\hline Germany & Ireland & 1999 & 2002 & 2.35 & 5.25 & 0.80 & 16.11 & 0.15 & -0.75 & -0.01 \\
\hline Germany & Italy & 1995 & 1998 & 6.09 & 17.28 & 0.35 & 10.65 & 0.08 & -3.10 & \\
\hline Germany & Italy & 1996 & 1999 & 7.66 & 61.09 & 0.18 & 13.12 & 0.18 & -1.87 & \\
\hline Germany & Italy & 1997 & 2000 & 8.99 & 35.63 & 0.02 & 0.65 & 0.04 & 2.92 & -1.31 \\
\hline Germany & Italy & 1998 & 2001 & 8.84 & 16.03 & 0.08 & 0.97 & 0.02 & -0.76 & -1.55 \\
\hline Germany & Italy & 1999 & 2002 & 4.49 & 8.40 & 0.84 & 9.47 & 0.06 & -2.85 & \\
\hline Germany & Netherlands & 1995 & 1998 & 6.83 & 10.76 & 0.80 & 4.69 & 0.05 & -0.20 & \\
\hline Germany & Netherlands & 1996 & 1999 & 11.91 & 16.70 & -0.78 & -3.75 & 0.13 & -3.02 & \\
\hline Germany & Netherlands & 1997 & 2000 & 7.22 & 44.08 & 0.50 & 11.92 & 0.17 & -2.60 & -1.63 \\
\hline Germany & Netherlands & 1998 & 2001 & 6.60 & 47.92 & 0.65 & 20.46 & 0.46 & -1.80 & \\
\hline Germany & Netherlands & 1999 & 2002 & 7.12 & 30.55 & 0.56 & 10.55 & 0.16 & -0.83 & \\
\hline Germany & Portugal & 1995 & 1998 & 6.72 & 39.54 & 0.29 & 18.55 & 0.38 & -2.01 & -2.19 \\
\hline Germany & Portugal & 1996 & 1999 & 7.79 & 67.22 & 0.17 & 13.11 & 0.29 & -2.13 & -2.07 \\
\hline Germany & Portugal & 1997 & 2000 & 8.95 & 36.94 & 0.03 & 0.82 & 0.04 & 2.88 & -1.20 \\
\hline Germany & Portugal & 1998 & 2001 & 8.88 & 16.36 & 0.08 & 0.90 & 0.03 & -0.74 & -1.52 \\
\hline Germany & Portugal & 1999 & 2002 & 6.58 & 10.83 & 0.54 & 4.90 & 0.11 & -1.74 & -1.23 \\
\hline Germany & Spain & 1995 & 1998 & 6.95 & 45.72 & 0.38 & 19.23 & 0.32 & -2.84 & -2.96 \\
\hline Germany & Spain & 1996 & 1999 & 7.66 & 97.27 & 0.26 & 20.92 & 0.71 & -3.15 & \\
\hline Germany & Spain & 1997 & 00 & 7.97 & .21 & 0.23 & 4.08 & 0.06 & 1.74 & 1.24 \\
\hline
\end{tabular}


Table A7: Rolling cointegration of short-term corporate lending rates

\begin{tabular}{|c|c|c|c|c|c|c|c|c|c|c|}
\hline & national interest rate $\mathrm{r}$ & sample & eriod & & coin & gration $\mathrm{r}$ & regression: & $=\mathrm{a}+\mathrm{b}$ & & \\
\hline $\mathrm{r}_{1}$ & $\mathrm{r}_{2}$ & start & end & $\mathrm{a}$ & t-stat(a) & $\mathrm{b}$ & t-stat(b) & DW & DF & $\mathrm{ADF}$ \\
\hline Germany & Spain & 1998 & 2001 & 6.48 & 16.39 & 0.59 & 7.39 & 0.17 & -1.56 & -1.09 \\
\hline Germany & Spain & 1999 & 2002 & 6.63 & 21.76 & 0.62 & 9.66 & 0.32 & -1.85 & -1.03 \\
\hline Germany & Australia & 1995 & 1998 & 3.15 & 6.04 & 0.66 & 12.78 & 0.17 & -2.09 & \\
\hline Germany & Australia & 1996 & 1999 & 4.90 & 21.75 & 0.46 & 19.38 & 0.61 & -3.32 & \\
\hline Germany & Australia & 1997 & 2000 & 3.90 & 8.04 & 0.58 & 10.86 & 0.25 & -1.10 & -1.98 \\
\hline Germany & Australia & 1998 & 2001 & 3.62 & 3.70 & 0.64 & 5.88 & 0.06 & -0.26 & -0.85 \\
\hline Germany & Australia & 1999 & 2002 & 5.43 & 5.35 & 0.46 & 4.05 & 0.04 & -1.00 & -1.27 \\
\hline Germany & Canada & 1995 & 1998 & 7.06 & 20.04 & 0.41 & 7.92 & 0.09 & -0.35 & \\
\hline Germany & Canada & 1996 & 1999 & 9.14 & 17.32 & 0.02 & 0.19 & 0.05 & -2.47 & \\
\hline Germany & Canada & 1997 & 2000 & 8.19 & 22.52 & 0.15 & 2.66 & 0.05 & 1.18 & -0.18 \\
\hline Germany & Canada & 1998 & 2001 & 9.32 & 14.46 & 0.01 & 0.08 & 0.03 & -0.58 & -1.82 \\
\hline Germany & Canada & 1999 & 2002 & 9.77 & 27.59 & -0.04 & -0.67 & 0.03 & -1.24 & -1.45 \\
\hline Germany & Japan & 1995 & 1998 & 7.13 & 18.78 & 1.49 & 7.12 & 0.07 & -0.80 & -1.23 \\
\hline Germany & Japan & 1996 & 1999 & 4.93 & 5.19 & 2.77 & 4.55 & 0.08 & -2.44 & \\
\hline Germany & Japan & 1997 & 2000 & 8.93 & 11.49 & 0.14 & 0.27 & 0.04 & 2.70 & -1.29 \\
\hline Germany & Japan & 1998 & 2001 & 10.50 & 8.66 & -0.78 & -0.94 & 0.03 & -0.58 & -2.22 \\
\hline Germany & Japan & 1999 & 2002 & 5.41 & 2.60 & 2.95 & 1.98 & 0.05 & -2.00 & \\
\hline Germany & Switzerland & 1995 & 1998 & 3.13 & 9.49 & 1.40 & 20.32 & 0.24 & -2.08 & \\
\hline Germany & Switzerland & 1996 & 1999 & 4.71 & 15.13 & 1.04 & 14.65 & 0.23 & -2.44 & \\
\hline Germany & Switzerland & 1997 & 2000 & 5.23 & 7.11 & 0.94 & 5.32 & 0.07 & 0.68 & 0.74 \\
\hline Germany & Switzerland & 1998 & 2001 & -0.49 & -0.70 & 2.38 & 14.10 & 0.38 & -2.92 & \\
\hline Germany & Switzerland & 1999 & 2002 & 3.45 & 3.55 & 1.48 & 6.27 & 0.08 & 0.11 & -0.10 \\
\hline Germany & United Kingdom & 1995 & 1998 & 12.97 & 8.20 & -0.42 & -2.03 & 0.03 & -1.13 & \\
\hline Germany & United Kingdom & 1996 & 1999 & 9.45 & 13.84 & -0.03 & -0.30 & 0.05 & -2.52 & \\
\hline Germany & United Kingdom & 1997 & 2000 & 8.94 & 16.80 & 0.03 & 0.39 & 0.04 & 2.73 & -1.36 \\
\hline Germany & United Kingdom & 1998 & 2001 & 10.43 & 17.80 & -0.15 & -1.84 & 0.04 & -0.68 & -2.14 \\
\hline Germany & United Kingdom & 1999 & 2002 & 9.90 & 17.14 & -0.06 & -0.63 & 0.03 & -1.20 & -2.17 \\
\hline Germany & USA & 1995 & 1998 & -4.98 & -1.65 & 1.74 & 4.88 & 0.12 & -2.22 & -1.17 \\
\hline Germany & USA & 1996 & 1999 & 6.24 & 2.75 & 0.36 & 1.32 & 0.05 & -2.20 & \\
\hline Germany & USA & 1997 & 2000 & 3.73 & 8.34 & 0.64 & 12.12 & 0.19 & -0.77 & -2.48 \\
\hline Germany & USA & 1998 & 2001 & 9.61 & 16.01 & -0.03 & -0.41 & 0.03 & -0.68 & -1.90 \\
\hline Germany & USA & 1999 & 2002 & 9.86 & 32.54 & -0.05 & -1.11 & 0.04 & -1.28 & -2.14 \\
\hline Ireland & Austria & 1995 & 1998 & 8.31 & 13.34 & 0.24 & 2.82 & 0.24 & -0.80 & -1.85 \\
\hline Ireland & Austria & 1996 & 1999 & 3.91 & 4.01 & 0.86 & 5.77 & 0.10 & -1.24 & -1.88 \\
\hline Ireland & Austria & 1997 & 2000 & -0.37 & -0.39 & 1.55 & 10.50 & 0.21 & -1.70 & \\
\hline Ireland & Austria & 1998 & 2001 & 0.03 & 0.04 & 1.48 & 11.25 & 0.24 & -1.98 & \\
\hline Ireland & Austria & 1999 & 2002 & 1.95 & 4.38 & 1.16 & 15.87 & 0.24 & -2.36 & \\
\hline Ireland & Belgium & 1995 & 1998 & 7.30 & 9.61 & 0.37 & 3.62 & 0.27 & -0.62 & -2.09 \\
\hline Ireland & Belgium & 1996 & 1999 & -5.00 & -2.09 & 2.05 & 6.07 & 0.23 & -1.57 & -1.97 \\
\hline Ireland & Belgium & 1997 & 2000 & 5.46 & 3.76 & 0.55 & 2.74 & 0.08 & -1.02 & -1.54 \\
\hline Ireland & Belgium & 1998 & 2001 & 4.88 & 5.39 & 0.58 & 4.90 & 0.11 & -1.89 & -2.60 \\
\hline Ireland & Belgium & 1999 & 2002 & 3.15 & 14.71 & 0.76 & 27.56 & 0.94 & -3.68 & -1.33 \\
\hline Ireland & Finland & 1995 & 1998 & 9.04 & 22.30 & 0.16 & 2.52 & 0.24 & -0.66 & -1.80 \\
\hline Ireland & Finland & 1996 & 1999 & 6.14 & 6.17 & 0.63 & 3.41 & 0.09 & -0.99 & -1.60 \\
\hline Ireland & Finland & 1997 & 2000 & 2.98 & 2.56 & 1.23 & 5.53 & 0.12 & -0.97 & -1.42 \\
\hline Ireland & Finland & 1998 & 2001 & 3.62 & 4.65 & 1.06 & 7.32 & 0.16 & -2.04 & -2.42 \\
\hline Ireland & Finland & 1999 & 2002 & 4.07 & 15.38 & 0.94 & 18.77 & 0.20 & -2.44 & \\
\hline Ireland & France & 1995 & 1998 & 9.76 & 29.64 & 0.04 & 0.89 & 0.22 & -0.54 & -1.59 \\
\hline Ireland & France & 1996 & 1999 & 8.26 & 17.54 & 0.22 & 2.71 & 0.08 & -0.69 & -1.35 \\
\hline Ireland & France & 1997 & 2000 & 6.06 & 8.41 & 0.66 & 4.72 & 0.12 & -1.36 & \\
\hline Ireland & France & 1998 & 2001 & 5.55 & 7.61 & 0.74 & 5.16 & 0.15 & -1.85 & \\
\hline Ireland & France & 1999 & 2002 & 4.80 & 16.91 & 0.84 & 14.94 & 0.66 & -3.03 & \\
\hline Ireland & Germany & 1995 & 1998 & 8.45 & 10.17 & 0.16 & 1.93 & 0.23 & -0.62 & -1.75 \\
\hline Ireland & Germany & 1996 & 1999 & 5.35 & 2.52 & 0.45 & 1.96 & 0.07 & -0.59 & -1.16 \\
\hline Ireland & Germany & 1997 & 2000 & 1.80 & 0.66 & 0.83 & 2.79 & 0.06 & -0.81 & -1.17 \\
\hline Ireland & Germany & 1998 & 2001 & 2.53 & 1.49 & 0.72 & 3.99 & 0.08 & -1.71 & -2.05 \\
\hline Ireland & Germany & 1999 & 2002 & -1.14 & -1.81 & 1.06 & 16.11 & 0.16 & -0.70 & 0.07 \\
\hline Ireland & Italy & 1995 & 1998 & 9.70 & 24.99 & 0.03 & 0.92 & 0.22 & -0.59 & -1.66 \\
\hline Ireland & Italy & 1996 & 1999 & 7.81 & 21.84 & 0.19 & 4.91 & 0.09 & -1.07 & -1.72 \\
\hline Ireland & Italy & 1997 & 2000 & 6.42 & 20.02 & 0.41 & 9.59 & 0.16 & -1.76 & -2.17 \\
\hline Ireland & Italy & 1998 & 2001 & 4.67 & 11.86 & 0.70 & 11.87 & 0.23 & -2.06 & -2.43 \\
\hline Ireland & Italy & 1999 & 2002 & 2.29 & 6.23 & 1.11 & 18.40 & 0.25 & -5.30 & \\
\hline Ireland & Netherlands & 1995 & 1998 & 8.26 & 21.96 & 0.49 & 4.81 & 0.33 & -0.65 & -1.97 \\
\hline Ireland & Netherlands & 1996 & 1999 & 8.39 & 6.41 & 0.33 & 0.86 & 0.06 & -0.37 & -0.91 \\
\hline Ireland & Netherlands & 1997 & 2000 & 8.80 & 12.21 & 0.16 & 0.87 & 0.06 & -0.93 & -1.34 \\
\hline Ireland & Netherlands & 1998 & 2001 & 7.19 & 13.38 & 0.49 & 3.97 & 0.08 & -1.90 & -2.17 \\
\hline Ireland & Netherlands & 1999 & 2002 & 5.77 & 54.51 & 0.75 & 31.18 & 0.62 & -2.65 & -0.45 \\
\hline Ireland & Portugal & 1995 & 1998 & 9.58 & 33.41 & 0.04 & 1.67 & 0.23 & -0.71 & -1.73 \\
\hline Ireland & Portugal & 1996 & 1999 & 8.08 & 23.53 & 0.17 & 4.35 & 0.10 & -1.07 & -1.49 \\
\hline Ireland & Portugal & 1997 & 2000 & 6.69 & 19.67 & 0.40 & 8.24 & 0.21 & -1.98 & \\
\hline Ireland & Portugal & 1998 & 2001 & 4.88 & 11.60 & 0.72 & 10.57 & 0.38 & -2.44 & -2.36 \\
\hline Ireland & Portugal & 1999 & 2002 & 4.39 & 8.30 & 0.84 & 8.79 & 0.38 & -3.02 & -2.43 \\
\hline Ireland & Spain & 1995 & 1998 & 9.71 & 36.22 & 0.05 & 1.32 & 0.21 & -0.56 & -1.68 \\
\hline Ireland & Spain & 1996 & 1999 & 8.35 & 22.39 & 0.19 & 3.24 & 0.07 & -0.77 & -1.32 \\
\hline Ireland & Spain & 1997 & 2000 & 5.36 & 12.20 & 0.79 & 9.38 & 0.26 & -2.67 & \\
\hline Ireland & Spain & 1998 & 2001 & 4.43 & 10.79 & 0.99 & 11.93 & 0.48 & -2.78 & \\
\hline Ireland & Spain & 1999 & 2002 & 5.15 & 24.70 & 0.82 & 18.73 & 1.15 & -4.34 & -2.72 \\
\hline Ireland & Australia & 1995 & 1998 & 10.01 & 15.07 & 0.00 & 0.06 & 0.21 & -0.39 & -1.56 \\
\hline Ireland & Australia & 1996 & 1999 & 7.71 & 7.20 & 0.19 & 1.69 & 0.07 & -0.58 & -1.17 \\
\hline Ireland & Australia & 1997 & 2000 & 4.70 & 2.50 & 0.52 & 2.51 & 0.08 & -0.96 & -1.59 \\
\hline Ireland & Australia & 1998 & 2001 & 4.65 & 2.71 & 0.52 & 2.71 & 0.09 & -1.67 & -2.30 \\
\hline Ireland & Australia & 1999 & 2002 & 2.85 & 2.80 & 0.69 & 6.06 & 0.11 & -1.15 & -1.81 \\
\hline Ireland & Canada & 1995 & 1998 & 9.33 & 30.64 & 0.11 & 2.42 & 0.23 & -0.27 & -1.48 \\
\hline Ireland & Canada & 1996 & 1999 & 11.39 & 14.11 & -0.31 & -2.35 & 0.10 & -1.04 & -1.45 \\
\hline Ireland & Canada & 1997 & 2000 & 11.00 & 13.35 & -0.25 & -1.94 & 0.09 & -0.77 & -1.50 \\
\hline Ireland & Canada & 1998 & 2001 & 7.96 & 8.89 & 0.20 & 1.49 & 0.07 & -1.53 & -2.24 \\
\hline
\end{tabular}


Table A7: Rolling cointegration of short-term corporate lending rates

\begin{tabular}{|c|c|c|c|c|c|c|c|c|c|c|}
\hline & national interest rate $\mathrm{r}$ & sample & seriod & & coil & egration & egression & $=\mathrm{a}+\mathrm{b}$ & & \\
\hline $\mathrm{r}_{1}$ & $\mathrm{r}_{2}$ & start & end & $\mathrm{a}$ & t-stat(a) & $\mathrm{b}$ & t-stat(b) & DW & DF & $\mathrm{ADF}$ \\
\hline Ireland & Canada & 1999 & 2002 & 8.34 & 20.94 & 0.11 & 1.73 & 0.04 & -1.18 & -2.51 \\
\hline Ireland & Japan & 1995 & 1998 & 9.08 & 30.80 & 0.54 & 3.35 & 0.27 & -0.92 & -2.24 \\
\hline Ireland & Japan & 1996 & 1999 & -1.72 & -2.10 & 7.20 & 13.75 & 0.32 & -1.91 & -2.50 \\
\hline Ireland & Japan & 1997 & 2000 & -0.57 & -0.67 & 6.62 & 11.74 & 0.28 & -1.76 & \\
\hline Ireland & Japan & 1998 & 2001 & 1.28 & 1.00 & 5.51 & 6.27 & 0.17 & -1.44 & \\
\hline Ireland & Japan & 1999 & 2002 & 1.70 & 0.75 & 5.22 & 3.23 & 0.11 & -2.13 & \\
\hline Ireland & Switzerland & 1995 & 1998 & 9.06 & 15.00 & 0.21 & 1.65 & 0.22 & -0.62 & -1.70 \\
\hline Ireland & Switzerland & 1996 & 1999 & 6.33 & 5.75 & 0.73 & 2.89 & 0.08 & -0.65 & -1.25 \\
\hline Ireland & Switzerland & 1997 & 2000 & 1.10 & 0.67 & 1.99 & 5.08 & 0.12 & -1.51 & -1.84 \\
\hline Ireland & Switzerland & 1998 & 2001 & -0.24 & -0.13 & 2.30 & 5.25 & 0.12 & -1.44 & -2.66 \\
\hline Ireland & Switzerland & 1999 & 2002 & 0.03 & 0.04 & 2.19 & 11.84 & 0.20 & -0.47 & \\
\hline Ireland & United Kingdom & 1995 & 1998 & 6.36 & 7.75 & 0.48 & 4.50 & 0.25 & -0.56 & \\
\hline Ireland & United Kingdom & 1996 & 1999 & 3.38 & 5.37 & 0.84 & 9.79 & 0.17 & -1.46 & \\
\hline Ireland & United Kingdom & 1997 & 2000 & 2.86 & 4.41 & 0.90 & 10.18 & 0.18 & -1.36 & \\
\hline Ireland & United Kingdom & 1998 & 2001 & 5.83 & 8.39 & 0.50 & 5.02 & 0.09 & -0.89 & \\
\hline Ireland & United Kingdom & 1999 & 2002 & 7.84 & 12.12 & 0.19 & 1.83 & 0.05 & -1.33 & -2.40 \\
\hline Ireland & USA & 1995 & 1998 & -3.13 & -2.89 & 1.56 & 12.17 & 0.78 & -3.30 & \\
\hline Ireland & USA & 1996 & 1999 & -10.90 & -4.90 & 2.47 & 9.17 & 0.16 & 0.61 & \\
\hline Ireland & USA & 1997 & 2000 & 4.07 & 2.20 & 0.63 & 2.90 & 0.05 & -0.94 & -1.25 \\
\hline Ireland & USA & 1998 & 2001 & 8.09 & 9.66 & 0.15 & 1.44 & 0.07 & -1.36 & -1.73 \\
\hline Ireland & USA & 1999 & 2002 & 8.58 & 24.61 & 0.06 & 1.29 & 0.05 & -1.10 & -2.49 \\
\hline Italy & Austria & 1995 & 1998 & -4.66 & -3.06 & 2.13 & 10.03 & 0.04 & -1.50 & -1.02 \\
\hline Italy & Austria & 1996 & 1999 & -16.92 & -19.36 & 3.97 & 29.58 & 0.34 & -2.15 & -2.57 \\
\hline Italy & Austria & 1997 & 2000 & -9.89 & -4.25 & 2.74 & 7.44 & 0.04 & 0.03 & \\
\hline Italy & Austria & 1998 & 2001 & -2.37 & -1.62 & 1.43 & 6.12 & 0.04 & -3.49 & \\
\hline Italy & Austria & 1999 & 2002 & -0.04 & -0.13 & 1.00 & 18.77 & 0.26 & -2.29 & \\
\hline Italy & Belgium & 1995 & 1998 & 0.00 & 0.00 & 1.41 & 3.40 & 0.05 & 0.52 & -0.41 \\
\hline Italy & Belgium & 1996 & 1999 & -30.00 & -3.77 & 5.49 & 4.89 & 0.09 & -0.78 & \\
\hline Italy & Belgium & 1997 & 2000 & 7.18 & 2.29 & 0.03 & 0.06 & 0.01 & -3.16 & -1.41 \\
\hline Italy & Belgium & 1998 & 2001 & 4.28 & 3.21 & 0.30 & 1.72 & 0.03 & -3.56 & -2.23 \\
\hline Italy & Belgium & 1999 & 2002 & 1.48 & 4.41 & 0.59 & 13.67 & 0.28 & -3.11 & \\
\hline Italy & Finland & 1995 & 1998 & 1.51 & 1.36 & 1.47 & 8.28 & 0.03 & 0.34 & -0.51 \\
\hline Italy & Finland & 1996 & 1999 & -11.65 & -7.64 & 3.81 & 13.50 & 0.07 & -1.37 & -1.44 \\
\hline Italy & Finland & 1997 & 2000 & -0.09 & -0.03 & 1.42 & 2.66 & 0.01 & -2.24 & -1.04 \\
\hline Italy & Finland & 1998 & 2001 & 1.98 & 1.60 & 0.85 & 3.72 & 0.02 & -4.74 & -2.65 \\
\hline Italy & Finland & 1999 & 2002 & 1.72 & 11.89 & 0.82 & 29.94 & 0.28 & -2.88 & \\
\hline Italy & France & 1995 & 1998 & 1.93 & 5.83 & 1.30 & 26.53 & 0.62 & -2.50 & \\
\hline Italy & France & 1996 & 1999 & -0.91 & -1.89 & 1.73 & 20.86 & 0.29 & -1.42 & -1.61 \\
\hline Italy & France & 1997 & 2000 & -0.23 & -0.17 & 1.49 & 5.70 & 0.07 & -0.54 & -0.86 \\
\hline Italy & France & 1998 & 2001 & 4.36 & 4.03 & 0.44 & 2.05 & 0.03 & -3.18 & \\
\hline Italy & France & 1999 & 2002 & 2.61 & 9.53 & 0.69 & 12.64 & 0.47 & -3.40 & \\
\hline Italy & Germany & 1995 & 1998 & -9.33 & -4.98 & 2.03 & 10.65 & 0.07 & -1.29 & \\
\hline Italy & Germany & 1996 & 1999 & -31.75 & -10.26 & 4.39 & 13.12 & 0.14 & -0.91 & \\
\hline Italy & Germany & 1997 & 2000 & 3.53 & 0.60 & 0.42 & 0.65 & 0.01 & -3.11 & -1.70 \\
\hline Italy & Germany & 1998 & 2001 & 4.24 & 1.76 & 0.25 & 0.97 & 0.03 & -3.36 & -2.09 \\
\hline Italy & Germany & 1999 & 2002 & -1.51 & -1.89 & 0.79 & 9.47 & 0.09 & -2.14 & \\
\hline Italy & Ireland & 1995 & 1998 & 5.08 & 0.86 & 0.54 & 0.92 & 0.01 & 1.25 & -1.57 \\
\hline Italy & Ireland & 1996 & 1999 & -8.21 & -2.36 & 1.79 & 4.91 & 0.04 & -1.54 & -1.90 \\
\hline Italy & Ireland & 1997 & 2000 & -8.06 & -4.99 & 1.64 & 9.59 & 0.11 & -2.51 & -2.42 \\
\hline Italy & Ireland & 1998 & 2001 & -3.38 & -4.02 & 1.07 & 11.87 & 0.18 & -2.65 & -2.72 \\
\hline Italy & Ireland & 1999 & 2002 & -1.08 & -2.79 & 0.79 & 18.40 & 0.25 & -5.02 & -2.68 \\
\hline Italy & Netherlands & 1995 & 1998 & 9.14 & 4.94 & 0.38 & 0.76 & 0.01 & 2.28 & 0.16 \\
\hline Italy & Netherlands & 1996 & 1999 & 22.34 & 6.40 & -3.95 & -3.89 & 0.07 & -1.86 & \\
\hline Italy & Netherlands & 1997 & 2000 & 10.23 & 7.35 & -0.75 & -2.09 & 0.02 & -1.75 & -2.03 \\
\hline Italy & Netherlands & 1998 & 2001 & 5.86 & 7.70 & 0.17 & 0.94 & 0.03 & -3.43 & -2.16 \\
\hline Italy & Netherlands & 1999 & 2002 & 3.40 & 24.32 & 0.61 & 19.17 & 0.49 & -4.28 & \\
\hline Italy & Portugal & 1995 & 1998 & 3.21 & 7.53 & 0.70 & 17.74 & 0.28 & -3.76 & -2.36 \\
\hline Italy & Portugal & 1996 & 1999 & 0.82 & 4.40 & 0.96 & 45.03 & 1.14 & -4.33 & -4.37 \\
\hline Italy & Portugal & 1997 & 2000 & 0.41 & 2.02 & 1.01 & 35.23 & 1.19 & -4.58 & \\
\hline Italy & Portugal & 1998 & 2001 & 0.29 & 1.29 & 1.03 & 28.19 & 1.18 & -4.34 & -2.75 \\
\hline Italy & Portugal & 1999 & 2002 & 1.59 & 5.11 & 0.80 & 14.36 & 0.75 & -3.13 & -2.51 \\
\hline Italy & Spain & 1995 & 1998 & 3.67 & 10.91 & 0.92 & 21.17 & 0.17 & -1.73 & \\
\hline Italy & Spain & 1996 & 1999 & 0.92 & 2.69 & 1.32 & 24.09 & 0.36 & -2.26 & -0.34 \\
\hline Italy & Spain & 1997 & 2000 & -1.22 & -1.56 & 1.68 & 11.13 & 0.24 & -1.37 & -0.46 \\
\hline Italy & Spain & 1998 & 2001 & 2.15 & 2.73 & 0.90 & 5.65 & 0.12 & -2.65 & -3.06 \\
\hline Italy & Spain & 1999 & 2002 & 2.93 & 12.85 & 0.66 & 13.71 & 0.74 & -4.13 & -3.06 \\
\hline Italy & Australia & 1995 & 1998 & -6.75 & -8.63 & 1.72 & 22.23 & 0.24 & -2.13 & \\
\hline Italy & Australia & 1996 & 1999 & -11.78 & -8.27 & 2.19 & 14.54 & 0.10 & -0.35 & \\
\hline Italy & Australia & 1997 & 2000 & -0.21 & -0.06 & 0.84 & 1.97 & 0.01 & -1.81 & -1.07 \\
\hline Italy & Australia & 1998 & 2001 & 5.18 & 2.29 & 0.15 & 0.61 & 0.03 & -3.25 & -2.20 \\
\hline Italy & Australia & 1999 & 2002 & 0.96 & 1.10 & 0.57 & 5.83 & 0.10 & -1.35 & -1.53 \\
\hline Italy & Canada & 1995 & 1998 & 7.54 & 6.16 & 0.46 & 2.51 & 0.02 & 2.10 & 0.59 \\
\hline Italy & Canada & 1996 & 1999 & 14.32 & 5.78 & -0.91 & -2.24 & 0.02 & -2.31 & -1.60 \\
\hline Italy & Canada & 1997 & 2000 & 15.51 & 12.76 & -1.28 & -6.77 & 0.09 & -1.11 & -1.47 \\
\hline Italy & Canada & 1998 & 2001 & 5.39 & 4.82 & 0.18 & 1.06 & 0.03 & -3.61 & -2.09 \\
\hline Italy & Canada & 1999 & 2002 & 5.16 & 16.13 & 0.15 & 2.78 & 0.05 & -0.74 & -1.53 \\
\hline Italy & Japan & 1995 & 1998 & 7.38 & 5.96 & 1.78 & 2.62 & 0.02 & 0.76 & -0.84 \\
\hline Italy & Japan & 1996 & 1999 & -22.58 & -7.00 & 20.12 & 9.76 & 0.07 & -1.95 & -1.94 \\
\hline Italy & Japan & 1997 & 2000 & -13.76 & -9.84 & 14.00 & 15.15 & 0.23 & -2.60 & -2.09 \\
\hline Italy & Japan & 1998 & 2001 & -6.15 & -5.91 & 8.75 & 12.23 & 0.32 & -2.41 & -3.19 \\
\hline Italy & Japan & 1999 & 2002 & -2.68 & -1.60 & 6.23 & 5.21 & 0.16 & -1.51 & -2.06 \\
\hline Italy & Switzerland & 1995 & 1998 & -5.01 & -5.11 & 3.28 & 15.96 & 0.12 & -1.75 & -1.30 \\
\hline Italy & Switzerland & 1996 & 1999 & -14.86 & -14.31 & 5.44 & 22.91 & 0.26 & -0.92 & -1.11 \\
\hline Italy & Switzerland & 1997 & 2000 & -12.52 & -4.34 & 4.76 & 6.91 & 0.07 & -0.25 & \\
\hline Italy & Switzerland & 1998 & 2001 & 0.52 & 0.19 & 1.46 & 2.25 & 0.03 & -2.83 & -3.37 \\
\hline Italy & Switzerland & 1999 & 02 & -1.75 & -3.04 & 1.90 & 13.51 & 0.27 & -1.25 & -1.08 \\
\hline
\end{tabular}


Table A7: Rolling cointegration of short-term corporate lending rates

\begin{tabular}{|c|c|c|c|c|c|c|c|c|c|c|}
\hline & national interest rate $\mathrm{r}$ & sample & eriod & & coin & gration $\mathrm{r}$ & egression: & $=\mathrm{a}+\mathrm{b}$ & & \\
\hline $\mathrm{r}_{1}$ & $\mathrm{r}_{2}$ & start & end & $\mathrm{a}$ & t-stat(a) & $\mathrm{b}$ & t-stat(b) & DW & DF & $\mathrm{ADF}$ \\
\hline Italy & United Kingdom & 1995 & 1998 & 25.97 & 7.97 & -2.03 & -4.75 & 0.08 & 1.26 & 0.35 \\
\hline Italy & United Kingdom & 1996 & 1999 & 4.55 & 1.37 & 0.59 & 1.29 & 0.01 & -1.61 & -1.68 \\
\hline Italy & United Kingdom & 1997 & 2000 & -2.35 & -1.27 & 1.34 & 5.28 & 0.04 & -4.01 & -3.16 \\
\hline Italy & United Kingdom & 1998 & 2001 & 1.03 & 1.52 & 0.80 & 8.25 & 0.07 & -1.43 & -1.41 \\
\hline Italy & United Kingdom & 1999 & 2002 & 4.51 & 8.73 & 0.25 & 2.99 & 0.05 & -0.75 & -1.44 \\
\hline Italy & USA & 1995 & 1998 & -13.21 & -1.60 & 2.80 & 2.87 & 0.04 & -0.33 & -1.41 \\
\hline Italy & USA & 1996 & 1999 & -22.76 & -2.18 & 3.82 & 3.03 & 0.03 & 0.06 & -0.98 \\
\hline Italy & USA & 1997 & 2000 & 6.82 & 1.69 & 0.07 & 0.14 & 0.01 & -3.19 & -1.40 \\
\hline Italy & USA & 1998 & 2001 & 5.33 & 5.13 & 0.15 & 1.19 & 0.03 & -3.26 & -2.28 \\
\hline Italy & USA & 1999 & 2002 & 5.45 & 19.12 & 0.08 & 2.14 & 0.05 & -0.80 & -1.75 \\
\hline Netherlands & Austria & 1995 & 1998 & 0.33 & 0.52 & 0.47 & 5.34 & 0.06 & -1.08 & \\
\hline Netherlands & Austria & 1996 & 1999 & 4.55 & 9.83 & -0.17 & -2.45 & 0.23 & -1.74 & \\
\hline Netherlands & Austria & 1997 & 2000 & 0.19 & 0.15 & 0.58 & 2.92 & 0.06 & -0.69 & -0.14 \\
\hline Netherlands & Austria & 1998 & 2001 & -4.55 & -4.60 & 1.41 & 8.92 & 0.12 & -1.90 & -2.04 \\
\hline Netherlands & Austria & 1999 & 2002 & -5.30 & -13.96 & 1.58 & 25.36 & 0.73 & -3.82 & \\
\hline Netherlands & Belgium & 1995 & 1998 & -2.40 & -4.85 & 0.81 & 12.34 & 0.54 & -2.74 & \\
\hline Netherlands & Belgium & 1996 & 1999 & 0.53 & 0.46 & 0.41 & 2.50 & 0.12 & -0.90 & \\
\hline Netherlands & Belgium & 1997 & 2000 & -3.99 & -9.09 & 1.08 & 17.88 & 0.41 & -3.15 & -2.93 \\
\hline Netherlands & Belgium & 1998 & 2001 & -3.23 & -11.71 & 0.98 & 27.27 & 0.65 & -2.69 & -0.68 \\
\hline Netherlands & Belgium & 1999 & 2002 & -3.02 & -7.43 & 0.95 & 18.08 & 0.40 & -1.68 & -0.14 \\
\hline Netherlands & Finland & 1995 & 1998 & 1.31 & 3.51 & 0.39 & 6.41 & 0.07 & -1.36 & -1.23 \\
\hline Netherlands & Finland & 1996 & 1999 & 4.13 & 9.95 & -0.13 & -1.71 & 0.21 & -1.62 & \\
\hline Netherlands & Finland & 1997 & 2000 & -2.18 & -2.75 & 1.15 & 7.62 & 0.09 & -2.05 & \\
\hline Netherlands & Finland & 1998 & 2001 & -3.00 & -6.39 & 1.35 & 15.53 & 0.25 & -2.19 & -1.86 \\
\hline Netherlands & Finland & 1999 & 2002 & -2.28 & -9.14 & 1.26 & 26.51 & 0.75 & -3.98 & \\
\hline Netherlands & France & 1995 & 1998 & 3.20 & 8.24 & 0.07 & 1.24 & 0.05 & -2.52 & -1.99 \\
\hline Netherlands & France & 1996 & 1999 & 4.25 & 28.33 & -0.15 & -5.65 & 0.33 & -2.35 & \\
\hline Netherlands & France & 1997 & 2000 & 2.52 & 3.79 & 0.26 & 2.00 & 0.07 & -0.59 & -0.95 \\
\hline Netherlands & France & 1998 & 2001 & -1.55 & -4.22 & 1.15 & 15.87 & 0.98 & -4.13 & \\
\hline Netherlands & France & 1999 & 2002 & -1.31 & -4.21 & 1.12 & 18.22 & 1.43 & -5.01 & \\
\hline Netherlands & Germany & 1995 & 1998 & -0.27 & -0.32 & 0.40 & 4.69 & 0.07 & -1.33 & \\
\hline Netherlands & Germany & 1996 & 1999 & 6.20 & 8.35 & -0.30 & -3.75 & 0.26 & -2.07 & \\
\hline Netherlands & Germany & 1997 & 2000 & -9.96 & -8.59 & 1.51 & 11.92 & 0.19 & -3.39 & \\
\hline Netherlands & Germany & 1998 & 2001 & -8.70 & -13.72 & 1.38 & 20.46 & 0.50 & -1.97 & \\
\hline Netherlands & Germany & 1999 & 2002 & -7.69 & -6.76 & 1.26 & 10.55 & 0.21 & -0.74 & \\
\hline Netherlands & Ireland & 1995 & 1998 & -3.24 & -2.25 & 0.69 & 4.81 & 0.16 & -1.85 & \\
\hline Netherlands & Ireland & 1996 & 1999 & 2.96 & 5.52 & 0.05 & 0.86 & 0.18 & -1.22 & \\
\hline Netherlands & Ireland & 1997 & 2000 & 2.89 & 2.64 & 0.10 & 0.87 & 0.06 & 0.46 & -0.67 \\
\hline Netherlands & Ireland & 1998 & 2001 & -0.55 & -0.45 & 0.52 & 3.97 & 0.07 & -1.54 & \\
\hline Netherlands & Ireland & 1999 & 2002 & -7.10 & -19.37 & 1.26 & 31.18 & 0.65 & -2.63 & -0.40 \\
\hline Netherlands & Italy & 1995 & 1998 & 3.33 & 7.19 & 0.03 & 0.76 & 0.05 & -2.58 & -2.12 \\
\hline Netherlands & Italy & 1996 & 1999 & 3.97 & 26.98 & -0.06 & -3.89 & 0.24 & -1.94 & \\
\hline Netherlands & Italy & 1997 & 2000 & 4.70 & 11.18 & -0.12 & -2.09 & 0.07 & 0.71 & -1.46 \\
\hline Netherlands & Italy & 1998 & 2001 & 3.50 & 4.36 & 0.11 & 0.94 & 0.06 & -1.12 & -2.19 \\
\hline Netherlands & Italy & 1999 & 2002 & -4.45 & -9.71 & 1.45 & 19.17 & 0.52 & -4.32 & \\
\hline Netherlands & Portugal & 1995 & 1998 & 2.81 & 8.63 & 0.08 & 2.73 & 0.05 & -1.62 & -1.69 \\
\hline Netherlands & Portugal & 1996 & 1999 & 3.95 & 29.52 & -0.06 & -4.13 & 0.26 & -2.06 & \\
\hline Netherlands & Portugal & 1997 & 2000 & 4.63 & 11.40 & -0.11 & -2.00 & 0.07 & 0.69 & -1.37 \\
\hline Netherlands & Portugal & 1998 & 2001 & 3.44 & 4.37 & 0.13 & 1.03 & 0.07 & -1.17 & -1.81 \\
\hline Netherlands & Portugal & 1999 & 2002 & -2.15 & -3.69 & 1.17 & 11.13 & 0.65 & -3.84 & \\
\hline Netherlands & Spain & 1995 & 1998 & 2.94 & 9.65 & 0.10 & 2.50 & 0.05 & -2.04 & -1.90 \\
\hline Netherlands & Spain & 1996 & 1999 & 3.96 & 29.10 & -0.09 & -4.10 & 0.27 & -2.20 & \\
\hline Netherlands & Spain & 1997 & 2000 & 3.35 & 5.69 & 0.10 & 0.85 & 0.06 & 0.22 & -0.52 \\
\hline Netherlands & Spain & 1998 & 2001 & -0.22 & -0.42 & 0.91 & 8.53 & 0.27 & -2.38 & -1.98 \\
\hline Netherlands & Spain & 1999 & 2002 & -0.79 & -3.45 & 1.08 & 22.47 & 1.76 & -5.99 & \\
\hline Netherlands & Australia & 1995 & 1998 & 2.88 & 3.68 & 0.08 & 1.03 & 0.05 & -2.38 & -2.23 \\
\hline Netherlands & Australia & 1996 & 1999 & 5.32 & 16.80 & -0.20 & -6.03 & 0.36 & -2.59 & \\
\hline Netherlands & Australia & 1997 & 2000 & -2.49 & -1.94 & 0.70 & 4.95 & 0.09 & -1.85 & -1.74 \\
\hline Netherlands & Australia & 1998 & 2001 & -5.50 & -4.51 & 1.08 & 8.01 & 0.17 & -1.40 & \\
\hline Netherlands & Australia & 1999 & 2002 & -4.54 & -3.82 & 0.99 & 7.44 & 0.18 & -1.18 & \\
\hline Netherlands & Canada & 1995 & 1998 & 1.50 & 7.51 & 0.33 & 11.12 & 0.19 & -1.90 & \\
\hline Netherlands & Canada & 1996 & 1999 & 2.35 & 8.19 & 0.18 & 3.78 & 0.22 & -1.54 & \\
\hline Netherlands & Canada & 1997 & 2000 & 0.57 & 1.21 & 0.52 & 7.03 & 0.13 & -0.20 & \\
\hline Netherlands & Canada & 1998 & 2001 & 2.61 & 2.88 & 0.25 & 1.82 & 0.06 & -0.81 & -1.70 \\
\hline Netherlands & Canada & 1999 & 2002 & 2.90 & 5.94 & 0.24 & 2.94 & 0.08 & -1.14 & -2.26 \\
\hline Netherlands & Japan & 1995 & 1998 & 1.65 & 6.76 & 1.14 & 8.48 & 0.17 & -1.25 & -1.50 \\
\hline Netherlands & Japan & 1996 & 1999 & 3.91 & 5.53 & -0.31 & -0.69 & 0.19 & -1.39 & \\
\hline Netherlands & Japan & 1997 & 2000 & 6.05 & 4.62 & -1.47 & -1.69 & 0.07 & 0.53 & -1.14 \\
\hline Netherlands & Japan & 1998 & 2001 & 5.52 & 3.11 & -0.88 & -0.72 & 0.07 & -0.92 & -2.40 \\
\hline Netherlands & Japan & 1999 & 2002 & -6.25 & -2.19 & 7.54 & 3.71 & 0.14 & -1.93 & \\
\hline Netherlands & Switzerland & 1995 & 1998 & 1.69 & 2.50 & 0.42 & 2.95 & 0.05 & -1.80 & -1.61 \\
\hline Netherlands & Switzerland & 1996 & 1999 & 5.35 & 14.79 & -0.44 & -5.35 & 0.32 & -2.26 & \\
\hline Netherlands & Switzerland & 1997 & 2000 & 1.37 & 0.86 & 0.59 & 1.57 & 0.06 & -0.33 & -0.65 \\
\hline Netherlands & Switzerland & 1998 & 2001 & -10.72 & -13.40 & 3.62 & 18.73 & 0.77 & -5.54 & \\
\hline Netherlands & Switzerland & 1999 & 2002 & -8.00 & -10.19 & 3.00 & 15.69 & 0.46 & -1.89 & \\
\hline Netherlands & United Kingdom & 1995 & 1998 & -0.23 & -0.23 & 0.51 & 3.84 & 0.07 & -3.76 & \\
\hline Netherlands & United Kingdom & 1996 & 1999 & 1.76 & 5.09 & 0.23 & 4.82 & 0.24 & -1.17 & \\
\hline Netherlands & United Kingdom & 1997 & 2000 & 3.71 & 4.02 & 0.02 & 0.14 & 0.06 & 0.59 & -0.78 \\
\hline Netherlands & United Kingdom & 1998 & 2001 & 5.00 & 5.70 & -0.11 & -0.86 & 0.07 & -0.90 & -2.60 \\
\hline Netherlands & United Kingdom & 1999 & 2002 & 1.84 & 2.34 & 0.40 & 3.16 & 0.08 & -1.35 & -2.04 \\
\hline Netherlands & USA & 1995 & 1998 & -9.40 & -5.18 & 1.54 & 7.21 & 0.26 & -2.29 & \\
\hline Netherlands & USA & 1996 & 1999 & 2.16 & 1.52 & 0.15 & 0.88 & 0.18 & -1.33 & \\
\hline Netherlands & USA & 1997 & 2000 & -5.09 & -5.74 & 1.05 & 10.09 & 0.18 & -1.47 & -1.91 \\
\hline Netherlands & USA & 1998 & 2001 & 3.30 & 3.81 & 0.12 & 1.11 & 0.06 & -0.98 & -2.28 \\
\hline Netherlands & USA & 1999 & 2002 & 3.27 & 7.58 & 0.14 & 2.47 & 0.08 & -1.10 & -2.47 \\
\hline Portugal & Austria & 1995 & 1998 & -12.74 & -10.96 & 3.25 & 20.09 & 0.37 & -1.96 & -1.30 \\
\hline
\end{tabular}


Table A7: Rolling cointegration of short-term corporate lending rates

\begin{tabular}{|c|c|c|c|c|c|c|c|c|c|c|}
\hline & national interest rate $\mathrm{r}$ & sample & seriod & & coil & gration & regression & $=\mathrm{a}+\mathrm{b}$ & & \\
\hline $\mathrm{r}_{1}$ & $\mathrm{r}_{2}$ & start & end & $\mathrm{a}$ & t-stat(a) & $\mathrm{b}$ & t-stat(b) & DW & DF & $\mathrm{ADF}$ \\
\hline Portugal & Austria & 1996 & 1999 & -17.95 & -17.43 & 4.05 & 25.63 & 0.62 & -2.90 & \\
\hline Portugal & Austria & 1997 & 2000 & -10.02 & -4.48 & 2.68 & 7.57 & 0.14 & -1.09 & \\
\hline Portugal & Austria & 1998 & 2001 & -2.56 & -1.90 & 1.38 & 6.44 & 0.15 & -2.97 & -3.41 \\
\hline Portugal & Austria & 1999 & 2002 & -1.14 & -2.66 & 1.10 & 15.61 & 1.03 & -4.44 & \\
\hline Portugal & Belgium & 1995 & 1998 & -10.51 & -3.04 & 2.80 & 6.10 & 0.19 & -0.91 & \\
\hline Portugal & Belgium & 1996 & 1999 & -29.24 & -3.47 & 5.32 & 4.46 & 0.13 & -1.12 & \\
\hline Portugal & Belgium & 1997 & 2000 & 6.39 & 2.09 & 0.07 & 0.16 & 0.05 & -2.42 & -1.93 \\
\hline Portugal & Belgium & 1998 & 2001 & 3.95 & 3.14 & 0.28 & 1.70 & 0.08 & -2.68 & -1.54 \\
\hline Portugal & Belgium & 1999 & 2002 & 1.36 & 2.34 & 0.54 & 7.21 & 0.34 & -2.22 & \\
\hline Portugal & Finland & 1995 & 1998 & -3.38 & -3.17 & 2.26 & 13.18 & 0.19 & -0.82 & -0.94 \\
\hline Portugal & Finland & 1996 & 1999 & -12.63 & -7.87 & 3.90 & 13.13 & 0.18 & -1.64 & -1.47 \\
\hline Portugal & Finland & 1997 & 2000 & -0.49 & -0.18 & 1.41 & 2.72 & 0.06 & -1.94 & \\
\hline Portugal & Finland & 1998 & 2001 & 1.74 & 1.49 & 0.81 & 3.75 & 0.10 & -2.96 & -2.11 \\
\hline Portugal & Finland & 1999 & 2002 & 0.92 & 3.32 & 0.88 & 16.67 & 1.09 & -4.69 & \\
\hline Portugal & France & 1995 & 1998 & -0.62 & -0.99 & 1.68 & 18.08 & 0.56 & -4.41 & \\
\hline Portugal & France & 1996 & 1999 & -1.77 & -3.91 & 1.80 & 22.97 & 0.59 & -2.62 & \\
\hline Portugal & France & 1997 & 2000 & -1.05 & -0.85 & 1.56 & 6.49 & 0.15 & -1.11 & \\
\hline Portugal & France & 1998 & 2001 & 3.78 & 3.75 & 0.46 & 2.29 & 0.09 & -2.62 & -1.49 \\
\hline Portugal & France & 1999 & 2002 & 1.85 & 5.25 & 0.74 & 10.56 & 0.78 & -4.03 & \\
\hline Portugal & Germany & 1995 & 1998 & -19.07 & -11.93 & 3.02 & 18.55 & 0.39 & -1.81 & -1.90 \\
\hline Portugal & Germany & 1996 & 1999 & -33.43 & -10.48 & 4.52 & 13.11 & 0.27 & -1.57 & \\
\hline Portugal & Germany & 1997 & 2000 & 2.18 & 0.38 & 0.51 & 0.82 & 0.05 & -2.25 & -1.77 \\
\hline Portugal & Germany & 1998 & 2001 & 4.04 & 1.78 & 0.22 & 0.90 & 0.09 & -2.53 & -1.52 \\
\hline Portugal & Germany & 1999 & 2002 & -0.58 & -0.46 & 0.64 & 4.90 & 0.26 & -1.71 & \\
\hline Portugal & Ireland & 1995 & 1998 & -2.43 & -0.31 & 1.29 & 1.67 & 0.05 & -1.31 & -1.00 \\
\hline Portugal & Ireland & 1996 & 1999 & -7.82 & -2.10 & 1.70 & 4.35 & 0.07 & -1.70 & -2.59 \\
\hline Portugal & Ireland & 1997 & 2000 & -7.29 & -4.22 & 1.50 & 8.24 & 0.20 & -2.90 & \\
\hline Portugal & Ireland & 1998 & 2001 & -3.00 & -3.48 & 0.98 & 10.57 & 0.39 & -3.03 & -2.15 \\
\hline Portugal & Ireland & 1999 & 2002 & -1.23 & -1.59 & 0.75 & 8.79 & 0.51 & -3.10 & \\
\hline Portugal & Italy & 1995 & 1998 & -2.66 & -3.54 & 1.25 & 17.74 & 0.31 & -4.49 & -3.17 \\
\hline Portugal & Italy & 1996 & 1999 & -0.65 & -3.13 & 1.02 & 45.03 & 1.16 & -4.46 & -4.63 \\
\hline Portugal & Italy & 1997 & 2000 & -0.15 & -0.71 & 0.95 & 35.23 & 1.23 & -4.82 & -4.91 \\
\hline Portugal & Italy & 1998 & 2001 & 0.07 & 0.31 & 0.92 & 28.19 & 1.24 & -4.52 & -4.43 \\
\hline Portugal & Italy & 1999 & 2002 & -0.61 & -1.42 & 1.02 & 14.36 & 0.88 & -3.41 & -2.55 \\
\hline Portugal & Netherlands & 1995 & 1998 & 4.26 & 1.85 & 1.69 & 2.73 & 0.05 & 0.21 & -0.01 \\
\hline Portugal & Netherlands & 1996 & 1999 & 22.90 & 6.47 & -4.26 & -4.13 & 0.11 & -2.16 & \\
\hline Portugal & Netherlands & 1997 & 2000 & 9.56 & 7.04 & -0.70 & -2.00 & 0.06 & -1.94 & -1.88 \\
\hline Portugal & Netherlands & 1998 & 2001 & 5.35 & 7.48 & 0.17 & 1.03 & 0.09 & -2.63 & -1.61 \\
\hline Portugal & Netherlands & 1999 & 2002 & 2.84 & 11.59 & 0.62 & 11.13 & 0.75 & -4.00 & \\
\hline Portugal & Spain & 1995 & 1998 & 1.23 & 2.99 & 1.23 & 23.21 & 0.73 & -3.74 & \\
\hline Portugal & Spain & 1996 & 1999 & 0.19 & 0.55 & 1.36 & 24.29 & 0.74 & -3.30 & \\
\hline Portugal & Spain & 1997 & 2000 & -1.55 & -2.12 & 1.65 & 11.64 & 0.37 & -1.88 & \\
\hline Portugal & Spain & 1998 & 2001 & 1.89 & 2.56 & 0.85 & 5.74 & 0.18 & -2.76 & -2.64 \\
\hline Portugal & Spain & 1999 & 2002 & 2.31 & 7.10 & 0.68 & 10.01 & 0.65 & -3.57 & \\
\hline Portugal & Australia & 1995 & 1998 & -12.24 & -10.10 & 2.26 & 18.87 & 0.34 & -2.58 & \\
\hline Portugal & Australia & 1996 & 1999 & -13.16 & -9.59 & 2.29 & 15.74 & 0.21 & -1.17 & -2.10 \\
\hline Portugal & Australia & 1997 & 2000 & -2.22 & -0.60 & 1.00 & 2.48 & 0.04 & -1.67 & \\
\hline Portugal & Australia & 1998 & 2001 & 3.72 & 1.76 & 0.26 & 1.12 & 0.08 & -2.67 & -1.63 \\
\hline Portugal & Australia & 1999 & 2002 & -0.65 & -0.71 & 0.69 & 6.75 & 0.32 & -2.06 & -1.86 \\
\hline Portugal & Canada & 1995 & 1998 & 4.20 & 2.87 & 0.95 & 4.41 & 0.07 & 0.08 & -0.08 \\
\hline Portugal & Canada & 1996 & 1999 & 13.85 & 5.41 & -0.91 & -2.18 & 0.04 & -2.22 & \\
\hline Portugal & Canada & 1997 & 2000 & 14.61 & 12.09 & -1.22 & -6.46 & 0.18 & -1.73 & \\
\hline Portugal & Canada & 1998 & 2001 & 4.32 & 4.18 & 0.27 & 1.71 & 0.09 & -3.06 & -1.80 \\
\hline Portugal & Canada & 1999 & 2002 & 3.98 & 12.78 & 0.26 & 5.07 & 0.27 & -1.67 & \\
\hline Portugal & Japan & 1995 & 1998 & 3.14 & 2.27 & 4.13 & 5.44 & 0.09 & -0.40 & -0.86 \\
\hline Portugal & Japan & 1996 & 1999 & -22.63 & -6.30 & 19.85 & 8.63 & 0.12 & -2.11 & \\
\hline Portugal & Japan & 1997 & 2000 & -12.83 & -8.02 & 13.06 & 12.36 & 0.30 & -3.01 & \\
\hline Portugal & Japan & 1998 & 2001 & -5.79 & -5.73 & 8.17 & 11.77 & 0.53 & -3.06 & \\
\hline Portugal & Japan & 1999 & 2002 & -4.98 & -2.77 & 7.51 & 5.85 & 0.47 & -2.28 & \\
\hline Portugal & Switzerland & 1995 & 1998 & -11.56 & -16.15 & 4.64 & 30.99 & 0.97 & -3.86 & -3.53 \\
\hline Portugal & Switzerland & 1996 & 1999 & -16.24 & -16.67 & 5.65 & 25.35 & 0.58 & -2.36 & -1.65 \\
\hline Portugal & Switzerland & 1997 & 2000 & -13.64 & -5.24 & 4.91 & 7.89 & 0.13 & -0.72 & -0.21 \\
\hline Portugal & Switzerland & 1998 & 2001 & 0.10 & 0.04 & 1.44 & 2.37 & 0.10 & -2.29 & -2.04 \\
\hline Portugal & Switzerland & 1999 & 2002 & -3.16 & -4.73 & 2.12 & 13.01 & 0.94 & -4.36 & \\
\hline Portugal & United Kingdom & 1995 & 1998 & 27.22 & 5.79 & -2.20 & -3.57 & 0.09 & -0.38 & -0.45 \\
\hline Portugal & United Kingdom & 1996 & 1999 & 5.21 & 1.51 & 0.43 & 0.91 & 0.03 & -1.42 & -1.66 \\
\hline Portugal & United Kingdom & 1997 & 2000 & -1.54 & -0.81 & 1.16 & 4.45 & 0.08 & -3.36 & \\
\hline Portugal & United Kingdom & 1998 & 2001 & 0.71 & 1.16 & 0.78 & 8.90 & 0.24 & -2.32 & -1.89 \\
\hline Portugal & United Kingdom & 1999 & 2002 & 2.82 & 5.76 & 0.44 & 5.57 & 0.31 & -1.85 & -1.97 \\
\hline Portugal & USA & 1995 & 1998 & -31.46 & -3.06 & 4.95 & 4.08 & 0.12 & -1.98 & -1.33 \\
\hline Portugal & USA & 1996 & 1999 & -20.97 & -1.91 & 3.54 & 2.68 & 0.05 & -0.61 & -1.49 \\
\hline Portugal & USA & 1997 & 2000 & 5.97 & 1.53 & 0.11 & 0.23 & 0.05 & -2.43 & -1.91 \\
\hline Portugal & USA & 1998 & 2001 & 4.30 & 4.48 & 0.22 & 1.87 & 0.09 & -2.66 & -1.77 \\
\hline Portugal & USA & 1999 & 2002 & 4.33 & 15.34 & 0.17 & 4.37 & 0.25 & -1.73 & -2.00 \\
\hline Spain & Austria & 1995 & 1998 & -10.19 & -9.69 & 2.47 & 16.90 & 0.17 & -1.91 & -2.34 \\
\hline Spain & Austria & 1996 & 1999 & -12.12 & -12.63 & 2.79 & 18.93 & 0.31 & -2.18 & \\
\hline Spain & Austria & 1997 & 2000 & -5.71 & -7.85 & 1.72 & 14.92 & 0.62 & -3.41 & \\
\hline Spain & Austria & 1998 & 2001 & -4.04 & -8.58 & 1.43 & 19.07 & 1.44 & -5.08 & -5.14 \\
\hline Spain & Austria & 1999 & 2002 & -3.53 & -7.62 & 1.36 & 17.87 & 1.31 & -5.15 & \\
\hline Spain & Belgium & 1995 & 1998 & -8.17 & -2.95 & 2.09 & 5.67 & 0.10 & -0.52 & -0.14 \\
\hline Spain & Belgium & 1996 & 1999 & -20.01 & -3.33 & 3.68 & 4.32 & 0.11 & -1.61 & \\
\hline Spain & Belgium & 1997 & 2000 & 0.64 & 0.44 & 0.62 & 3.09 & 0.12 & -2.80 & \\
\hline Spain & Belgium & 1998 & 2001 & -0.49 & -0.87 & 0.71 & 9.58 & 0.43 & -2.59 & \\
\hline Spain & Belgium & 1999 & 2002 & -1.53 & -3.41 & 0.81 & 14.01 & 0.81 & -3.30 & -2.06 \\
\hline Spain & Finland & 1995 & 1998 & -3.54 & -4.72 & 1.80 & 14.90 & 0.11 & -0.86 & \\
\hline Spain & Finland & 1996 & 1999 & -9.52 & -10.41 & 2.88 & 17.04 & 0.23 & -1.69 & \\
\hline
\end{tabular}


Table A7: Rolling cointegration of short-term corporate lending rates

\begin{tabular}{|c|c|c|c|c|c|c|c|c|c|c|}
\hline & national interest rate $\mathrm{r}$ & sample & seriod & & coil & gration & regression: & $=\mathrm{a}+\mathrm{b}$ & & \\
\hline $\mathrm{r}_{1}$ & $\mathrm{r}_{2}$ & start & end & $\mathrm{a}$ & t-stat(a) & $\mathrm{b}$ & t-stat(b) & DW & DF & $\mathrm{ADF}$ \\
\hline Spain & Finland & 1997 & 2000 & -1.86 & -1.63 & 1.33 & 6.14 & 0.17 & -2.65 & -2.13 \\
\hline Spain & Finland & 1998 & 2001 & -1.31 & -3.22 & 1.16 & 15.38 & 0.86 & -3.82 & \\
\hline Spain & Finland & 1999 & 2002 & -0.92 & -2.89 & 1.08 & 17.84 & 1.11 & -4.60 & -2.89 \\
\hline Spain & France & 1995 & 1998 & -1.28 & -3.00 & 1.33 & 21.01 & 0.61 & -3.42 & \\
\hline Spain & France & 1996 & 1999 & -1.18 & -3.82 & 1.27 & 23.74 & 0.55 & -3.71 & -3.58 \\
\hline Spain & France & 1997 & 2000 & -0.11 & -0.25 & 1.03 & 11.94 & 0.52 & -2.67 & \\
\hline Spain & France & 1998 & 2001 & 0.43 & 0.95 & 0.89 & 9.92 & 0.59 & -2.93 & \\
\hline Spain & France & 1999 & 2002 & -0.22 & -0.75 & 0.99 & 17.14 & 1.48 & -5.19 & \\
\hline Spain & Germany & 1995 & 1998 & -15.57 & -12.94 & 2.36 & 19.23 & 0.32 & -2.39 & -2.35 \\
\hline Spain & Germany & 1996 & 1999 & -25.64 & -16.94 & 3.42 & 20.92 & 0.69 & -3.07 & \\
\hline Spain & Germany & 1997 & 2000 & -5.44 & -2.10 & 1.15 & 4.08 & 0.12 & -2.35 & -1.44 \\
\hline Spain & Germany & 1998 & 2001 & -3.72 & -3.18 & 0.92 & 7.39 & 0.32 & -2.10 & -2.19 \\
\hline Spain & Germany & 1999 & 2002 & -5.65 & -5.25 & 1.09 & 9.66 & 0.45 & -2.14 & -1.12 \\
\hline Spain & Ireland & 1995 & 1998 & -0.58 & -0.09 & 0.80 & 1.32 & 0.02 & -0.64 & -1.38 \\
\hline Spain & Ireland & 1996 & 1999 & -3.13 & -1.11 & 0.96 & 3.24 & 0.04 & -2.67 & -1.93 \\
\hline Spain & Ireland & 1997 & 2000 & -2.68 & -3.21 & 0.83 & 9.38 & 0.30 & -3.62 & -3.68 \\
\hline Spain & Ireland & 1998 & 2001 & -2.19 & -3.66 & 0.76 & 11.93 & 0.58 & -2.86 & -1.92 \\
\hline Spain & Ireland & 1999 & 2002 & -5.02 & -9.62 & 1.08 & 18.73 & 1.27 & -4.61 & -2.91 \\
\hline Spain & Italy & 1995 & 1998 & -2.93 & -5.85 & 0.99 & 21.17 & 0.18 & -2.29 & \\
\hline Spain & Italy & 1996 & 1999 & -0.21 & -0.77 & 0.70 & 24.09 & 0.38 & -2.85 & -0.87 \\
\hline Spain & Italy & 1997 & 2000 & 1.91 & 6.49 & 0.43 & 11.13 & 0.34 & -1.90 & -1.06 \\
\hline Spain & Italy & 1998 & 2001 & 1.91 & 3.58 & 0.46 & 5.65 & 0.26 & -1.95 & -1.43 \\
\hline Spain & Italy & 1999 & 2002 & -2.67 & -4.93 & 1.23 & 13.71 & 0.86 & -4.48 & -3.36 \\
\hline Spain & Netherlands & 1995 & 1998 & 3.02 & 1.66 & 1.22 & 2.50 & 0.03 & 0.36 & -0.57 \\
\hline Spain & Netherlands & 1996 & 1999 & 16.20 & 6.47 & -2.99 & -4.10 & 0.13 & -3.04 & \\
\hline Spain & Netherlands & 1997 & 2000 & 4.50 & 6.12 & 0.16 & 0.85 & 0.10 & -2.54 & -1.86 \\
\hline Spain & Netherlands & 1998 & 2001 & 2.05 & 6.02 & 0.67 & 8.53 & 0.37 & -2.64 & \\
\hline Spain & Netherlands & 1999 & 2002 & 1.07 & 6.45 & 0.85 & 22.47 & 1.85 & -6.29 & \\
\hline Spain & Portugal & 1995 & 1998 & -0.33 & -0.95 & 0.75 & 23.21 & 0.72 & -3.55 & -3.31 \\
\hline Spain & Portugal & 1996 & 1999 & 0.30 & 1.23 & 0.68 & 24.29 & 0.75 & -3.62 & \\
\hline Spain & Portugal & 1997 & 2000 & 2.00 & 7.27 & 0.45 & 11.64 & 0.42 & -2.06 & \\
\hline Spain & Portugal & 1998 & 2001 & 1.94 & 3.71 & 0.49 & 5.74 & 0.26 & -2.02 & \\
\hline Spain & Portugal & 1999 & 2002 & -0.83 & -1.49 & 1.01 & 10.01 & 0.64 & -3.70 & \\
\hline Spain & Australia & 1995 & 1998 & -10.39 & -12.30 & 1.78 & 21.29 & 0.40 & -2.27 & \\
\hline Spain & Australia & 1996 & 1999 & -9.83 & -13.56 & 1.68 & 21.92 & 0.58 & -3.21 & -2.38 \\
\hline Spain & Australia & 1997 & 2000 & -4.06 & -2.65 & 1.01 & 6.00 & 0.21 & -2.17 & -0.81 \\
\hline Spain & Australia & 1998 & 2001 & -2.29 & -1.87 & 0.80 & 5.89 & 0.32 & -2.23 & \\
\hline Spain & Australia & 1999 & 2002 & -3.49 & -3.49 & 0.92 & 8.24 & 0.45 & -2.34 & \\
\hline Spain & Canada & 1995 & 1998 & 2.25 & 2.05 & 0.79 & 4.89 & 0.05 & 0.41 & -0.13 \\
\hline Spain & Canada & 1996 & 1999 & 7.87 & 4.19 & -0.31 & -1.02 & 0.04 & -3.04 & -1.33 \\
\hline Spain & Canada & 1997 & 2000 & 7.55 & 9.48 & -0.38 & -3.08 & 0.15 & -1.60 & -1.62 \\
\hline Spain & Canada & 1998 & 2001 & 2.91 & 3.88 & 0.30 & 2.69 & 0.18 & -1.63 & \\
\hline Spain & Canada & 1999 & 2002 & 3.44 & 7.97 & 0.22 & 3.07 & 0.19 & -1.75 & -2.03 \\
\hline Spain & Japan & 1995 & 1998 & 2.81 & 2.37 & 2.63 & 4.06 & 0.04 & -0.30 & -1.59 \\
\hline Spain & Japan & 1996 & 1999 & -13.16 & -4.41 & 12.26 & 6.42 & 0.08 & -2.67 & -1.87 \\
\hline Spain & Japan & 1997 & 2000 & -3.32 & -2.73 & 5.60 & 6.96 & 0.23 & -2.30 & -1.53 \\
\hline Spain & Japan & 1998 & 2001 & 0.74 & 0.53 & 2.87 & 2.98 & 0.21 & -1.56 & -1.82 \\
\hline Spain & Japan & 1999 & 2002 & -3.89 & -1.50 & 6.16 & 3.33 & 0.26 & -2.31 & \\
\hline Spain & Switzerland & 1995 & 1998 & -9.31 & -12.49 & 3.54 & 22.69 & 0.38 & -2.28 & \\
\hline Spain & Switzerland & 1996 & 1999 & -11.25 & -14.82 & 3.96 & 22.79 & 0.63 & -3.79 & \\
\hline Spain & Switzerland & 1997 & 2000 & -7.72 & -8.67 & 3.07 & 14.44 & 0.71 & -3.09 & \\
\hline Spain & Switzerland & 1998 & 2001 & -6.59 & -6.04 & 2.78 & 10.54 & 0.68 & -3.05 & \\
\hline Spain & Switzerland & 1999 & 2002 & -5.82 & -7.12 & 2.57 & 12.93 & 0.83 & -3.13 & -1.90 \\
\hline Spain & United Kingdom & 1995 & 1998 & 19.58 & 5.26 & -1.59 & -3.26 & 0.05 & 0.09 & -0.83 \\
\hline Spain & United Kingdom & 1996 & 1999 & 5.03 & 2.05 & 0.13 & 0.38 & 0.03 & -2.63 & -1.05 \\
\hline Spain & United Kingdom & 1997 & 2000 & 1.06 & 1.03 & 0.56 & 3.96 & 0.14 & -2.86 & -1.68 \\
\hline Spain & United Kingdom & 1998 & 2001 & 2.82 & 4.07 & 0.30 & 3.03 & 0.18 & -1.48 & \\
\hline Spain & United Kingdom & 1999 & 2002 & 2.56 & 3.65 & 0.36 & 3.13 & 0.20 & -1.89 & -1.45 \\
\hline Spain & USA & 1995 & 1998 & -22.70 & -2.77 & 3.56 & 3.69 & 0.09 & -1.36 & \\
\hline Spain & USA & 1996 & 1999 & -10.89 & -1.37 & 2.04 & 2.12 & 0.05 & -1.85 & \\
\hline Spain & USA & 1997 & 2000 & -0.82 & -0.44 & 0.70 & 3.21 & 0.12 & -2.92 & -3.07 \\
\hline Spain & USA & 1998 & 2001 & 3.36 & 4.69 & 0.19 & 2.18 & 0.18 & -1.51 & -1.64 \\
\hline Spain & USA & 1999 & 2002 & 3.78 & 9.90 & 0.13 & 2.56 & 0.19 & -1.66 & -2.25 \\
\hline Australia & Austria & 1995 & 1998 & 1.39 & 1.74 & 1.21 & 10.87 & 0.09 & -1.12 & -1.43 \\
\hline Australia & Austria & 1996 & 1999 & 0.07 & 0.09 & 1.44 & 11.13 & 0.13 & -1.14 & \\
\hline Australia & Austria & 1997 & 2000 & 3.54 & 4.38 & 0.88 & 6.84 & 0.13 & -1.00 & -1.74 \\
\hline Australia & Austria & 1998 & 2001 & 3.57 & 4.33 & 0.87 & 6.58 & 0.13 & -1.53 & -2.01 \\
\hline Australia & Austria & 1999 & 2002 & 2.83 & 4.54 & 1.01 & 9.86 & 0.20 & -1.63 & -1.68 \\
\hline Australia & Belgium & 1995 & 1998 & 3.38 & 2.04 & 0.89 & 4.02 & 0.07 & -0.29 & -0.21 \\
\hline Australia & Belgium & 1996 & 1999 & -0.87 & -0.23 & 1.45 & 2.74 & 0.05 & -1.17 & -1.88 \\
\hline Australia & Belgium & 1997 & 2000 & 3.92 & 5.46 & 0.71 & 7.20 & 0.14 & -2.32 & -3.12 \\
\hline Australia & Belgium & 1998 & 2001 & 5.36 & 8.99 & 0.48 & 6.13 & 0.12 & -0.70 & -1.35 \\
\hline Australia & Belgium & 1999 & 2002 & 5.50 & 7.86 & 0.45 & 4.98 & 0.09 & -1.05 & -1.55 \\
\hline Australia & Finland & 1995 & 1998 & 4.84 & 8.35 & 0.85 & 9.08 & 0.07 & -0.43 & -0.81 \\
\hline Australia & Finland & 1996 & 1999 & 1.33 & 1.81 & 1.50 & 11.00 & 0.12 & -1.31 & \\
\hline Australia & Finland & 1997 & 2000 & 4.20 & 5.98 & 0.93 & 6.96 & 0.14 & -1.67 & -2.47 \\
\hline Australia & Finland & 1998 & 2001 & 5.10 & 8.65 & 0.72 & 6.62 & 0.12 & -1.21 & \\
\hline Australia & Finland & 1999 & 2002 & 5.25 & 10.16 & 0.71 & 7.26 & 0.13 & -1.35 & -1.81 \\
\hline Australia & France & 1995 & 1998 & 5.32 & 24.77 & 0.71 & 22.52 & 0.64 & -3.54 & \\
\hline Australia & France & 1996 & 1999 & 5.29 & 34.00 & 0.73 & 27.06 & 0.68 & -3.17 & \\
\hline Australia & France & 1997 & 2000 & 5.70 & 18.88 & 0.66 & 11.30 & 0.46 & -2.33 & \\
\hline Australia & France & 1998 & 2001 & 5.71 & 12.83 & 0.65 & 7.43 & 0.30 & -1.77 & -2.20 \\
\hline Australia & France & 1999 & 2002 & 5.65 & 12.60 & 0.66 & 7.46 & 0.29 & -1.89 & \\
\hline Australia & Germany & 1995 & 1998 & -1.52 & -1.67 & 1.18 & 12.78 & 0.17 & -1.48 & \\
\hline Australia & Germany & 1996 & 1999 & -8.42 & -9.15 & 1.93 & 19.38 & 0.59 & -3.00 & \\
\hline Australia & Germany & 1997 & 2000 & -2.29 & -2.19 & 1.24 & 10.86 & 0.28 & -2.15 & -3.28 \\
\hline
\end{tabular}


Table A7: Rolling cointegration of short-term corporate lending rates

\begin{tabular}{|c|c|c|c|c|c|c|c|c|c|c|}
\hline & national interest rate $\mathrm{r}$ & sample & eriod & & coin & gration & egression: & $=\mathrm{a}+\mathrm{b}$ & & \\
\hline $\mathrm{r}_{1}$ & $\mathrm{r}_{2}$ & start & end & $\mathrm{a}$ & t-stat(a) & $\mathrm{b}$ & t-stat(b) & DW & DF & $\mathrm{ADF}$ \\
\hline Australia & Germany & 1998 & 2001 & 2.70 & 2.52 & 0.67 & 5.88 & 0.11 & -0.52 & -1.33 \\
\hline Australia & Germany & 1999 & 2002 & 3.49 & 2.57 & 0.58 & 4.05 & 0.08 & -0.95 & -1.90 \\
\hline Australia & Ireland & 1995 & 1998 & 9.84 & 2.96 & 0.02 & 0.06 & 0.02 & -0.01 & \\
\hline Australia & Ireland & 1996 & 1999 & 6.49 & 3.77 & 0.30 & 1.69 & 0.03 & -1.79 & \\
\hline Australia & Ireland & 1997 & 2000 & 6.88 & 7.88 & 0.23 & 2.51 & 0.08 & -0.57 & -1.55 \\
\hline Australia & Ireland & 1998 & 2001 & 6.51 & 7.08 & 0.27 & 2.71 & 0.09 & -1.11 & -1.75 \\
\hline Australia & Ireland & 1999 & 2002 & 3.14 & 3.26 & 0.65 & 6.06 & 0.13 & -1.25 & -2.20 \\
\hline Australia & Italy & 1995 & 1998 & 4.44 & 17.35 & 0.53 & 22.23 & 0.25 & -2.57 & \\
\hline Australia & Italy & 1996 & 1999 & 6.09 & 25.84 & 0.37 & 14.54 & 0.12 & -0.93 & \\
\hline Australia & Italy & 1997 & 2000 & 8.39 & 23.53 & 0.09 & 1.97 & 0.07 & 0.07 & -1.76 \\
\hline Australia & Italy & 1998 & 2001 & 8.64 & 15.21 & 0.05 & 0.61 & 0.07 & -0.83 & -1.88 \\
\hline Australia & Italy & 1999 & 2002 & 4.44 & 5.68 & 0.75 & 5.83 & 0.11 & -1.65 & -1.78 \\
\hline Australia & Netherlands & 1995 & 1998 & 9.00 & 8.81 & 0.28 & 1.03 & 0.02 & 0.33 & -0.01 \\
\hline Australia & Netherlands & 1996 & 1999 & 16.86 & 13.57 & -2.18 & -6.03 & 0.21 & -2.84 & \\
\hline Australia & Netherlands & 1997 & 2000 & 7.16 & 18.26 & 0.50 & 4.95 & 0.10 & -2.51 & -3.03 \\
\hline Australia & Netherlands & 1998 & 2001 & 6.71 & 23.16 & 0.54 & 8.01 & 0.18 & -1.27 & \\
\hline Australia & Netherlands & 1999 & 2002 & 6.59 & 20.26 & 0.55 & 7.44 & 0.17 & -1.35 & \\
\hline Australia & Portugal & 1995 & 1998 & 5.94 & 26.51 & 0.39 & 18.87 & 0.33 & -2.36 & \\
\hline Australia & Portugal & 1996 & 1999 & 6.32 & 31.03 & 0.37 & 15.74 & 0.21 & -1.34 & -2.77 \\
\hline Australia & Portugal & 1997 & 2000 & 8.26 & 24.59 & 0.12 & 2.48 & 0.06 & 0.41 & -1.54 \\
\hline Australia & Portugal & 1998 & 2001 & 8.38 & 15.13 & 0.10 & 1.12 & 0.07 & -0.92 & -1.36 \\
\hline Australia & Portugal & 1999 & 2002 & 4.98 & 8.36 & 0.72 & 6.75 & 0.21 & -2.02 & -1.92 \\
\hline Australia & Spain & 1995 & 1998 & 6.22 & 33.50 & 0.51 & 21.29 & 0.40 & -2.24 & \\
\hline Australia & Spain & 1996 & 1999 & 6.15 & 39.91 & 0.54 & 21.92 & 0.57 & -2.97 & -2.44 \\
\hline Australia & Spain & 1997 & 2000 & 6.85 & 18.27 & 0.43 & 6.00 & 0.18 & -0.89 & \\
\hline Australia & Spain & 1998 & 2001 & 6.35 & 14.06 & 0.54 & 5.89 & 0.22 & -1.80 & \\
\hline Australia & Spain & 1999 & 2002 & 5.89 & 15.61 & 0.65 & 8.24 & 0.35 & -2.08 & \\
\hline Australia & Canada & 1995 & 1998 & 7.89 & 12.19 & 0.33 & 3.40 & 0.04 & 0.48 & 0.06 \\
\hline Australia & Canada & 1996 & 1999 & 10.57 & 9.94 & -0.19 & -1.12 & 0.03 & -2.26 & \\
\hline Australia & Canada & 1997 & 2000 & 8.89 & 15.54 & 0.03 & 0.33 & 0.07 & -0.48 & -2.18 \\
\hline Australia & Canada & 1998 & 2001 & 6.22 & 12.04 & 0.42 & 5.40 & 0.12 & -0.88 & \\
\hline Australia & Canada & 1999 & 2002 & 7.19 & 24.44 & 0.30 & 6.22 & 0.09 & -1.65 & \\
\hline Australia & Japan & 1995 & 1998 & 7.84 & 11.91 & 1.24 & 3.42 & 0.04 & -0.10 & \\
\hline Australia & Japan & 1996 & 1999 & 0.87 & 0.44 & 5.46 & 4.34 & 0.05 & -1.67 & -1.82 \\
\hline Australia & Japan & 1997 & 2000 & 8.48 & 7.46 & 0.39 & 0.52 & 0.07 & -0.31 & -2.03 \\
\hline Australia & Japan & 1998 & 2001 & 8.82 & 7.04 & 0.11 & 0.13 & 0.08 & -0.76 & -1.93 \\
\hline Australia & Japan & 1999 & 2002 & -0.08 & -0.04 & 6.47 & 4.45 & 0.15 & -2.47 & -1.75 \\
\hline Australia & Switzerland & 1995 & 1998 & 1.31 & 2.57 & 1.84 & 17.31 & 0.27 & -1.80 & -2.31 \\
\hline Australia & Switzerland & 1996 & 1999 & -0.35 & -0.78 & 2.24 & 21.75 & 0.48 & -2.61 & -2.90 \\
\hline Australia & Switzerland & 1997 & 2000 & 1.60 & 1.97 & 1.79 & 9.19 & 0.24 & -1.62 & \\
\hline Australia & Switzerland & 1998 & 2001 & 0.31 & 0.29 & 2.10 & 8.32 & 0.25 & -2.08 & -1.76 \\
\hline Australia & Switzerland & 1999 & 2002 & 1.85 & 1.77 & 1.74 & 6.82 & 0.18 & -0.92 & -2.19 \\
\hline Australia & United Kingdom & 1995 & 1998 & 19.36 & 11.22 & -1.22 & -5.42 & 0.10 & 0.07 & -0.66 \\
\hline Australia & United Kingdom & 1996 & 1999 & 10.94 & 7.95 & -0.21 & -1.13 & 0.03 & -1.72 & \\
\hline Australia & United Kingdom & 1997 & 2000 & 9.35 & 11.99 & -0.04 & -0.36 & 0.07 & -0.39 & -2.15 \\
\hline Australia & United Kingdom & 1998 & 2001 & 8.41 & 13.65 & 0.08 & 0.95 & 0.07 & -0.90 & -1.94 \\
\hline Australia & United Kingdom & 1999 & 2002 & 6.13 & 12.42 & 0.47 & 5.83 & 0.10 & -1.95 & -2.08 \\
\hline Australia & USA & 1995 & 1998 & -0.56 & -0.12 & 1.25 & 2.24 & 0.04 & -0.96 & -0.71 \\
\hline Australia & USA & 1996 & 1999 & 3.86 & 0.83 & 0.67 & 1.19 & 0.03 & -1.49 & \\
\hline Australia & USA & 1997 & 2000 & 1.89 & 2.30 & 0.84 & 8.74 & 0.17 & -2.71 & -3.28 \\
\hline Australia & USA & 1998 & 2001 & 6.31 & 13.42 & 0.33 & 5.75 & 0.09 & -0.80 & -1.05 \\
\hline Australia & USA & 1999 & 2002 & 7.52 & 28.51 & 0.20 & 5.69 & 0.09 & -1.21 & -2.13 \\
\hline Canada & Austria & 1995 & 1998 & -3.80 & -2.73 & 1.45 & 7.50 & 0.09 & -0.43 & \\
\hline Canada & Austria & 1996 & 1999 & 7.95 & 6.04 & -0.30 & -1.46 & 0.11 & -1.94 & -1.93 \\
\hline Canada & Austria & 1997 & 2000 & 8.32 & 4.43 & -0.31 & -1.06 & 0.07 & -0.97 & -1.28 \\
\hline Canada & Austria & 1998 & 2001 & 1.86 & 1.20 & 0.75 & 3.04 & 0.12 & 0.49 & \\
\hline Canada & Austria & 1999 & 2002 & -3.22 & -1.56 & 1.50 & 4.44 & 0.04 & -0.77 & \\
\hline Canada & Belgium & 1995 & 1998 & -9.54 & -8.18 & 2.15 & 13.88 & 0.52 & -2.55 & \\
\hline Canada & Belgium & 1996 & 1999 & 1.00 & 0.30 & 0.71 & 1.53 & 0.09 & -1.23 & \\
\hline Canada & Belgium & 1997 & 2000 & -0.05 & -0.03 & 0.88 & 4.43 & 0.08 & -1.81 & \\
\hline Canada & Belgium & 1998 & 2001 & 5.56 & 4.73 & 0.13 & 0.86 & 0.11 & 1.07 & -0.02 \\
\hline Canada & Belgium & 1999 & 2002 & 4.73 & 2.44 & 0.16 & 0.62 & 0.03 & -0.28 & -1.29 \\
\hline Canada & Finland & 1995 & 1998 & -0.84 & -1.14 & 1.21 & 10.22 & 0.12 & -0.86 & -1.39 \\
\hline Canada & Finland & 1996 & 1999 & 5.16 & 4.43 & 0.16 & 0.76 & 0.10 & -1.29 & \\
\hline Canada & Finland & 1997 & 2000 & 3.14 & 1.97 & 0.61 & 2.02 & 0.07 & -1.83 & \\
\hline Canada & Finland & 1998 & 2001 & 4.00 & 3.46 & 0.48 & 2.23 & 0.12 & 0.81 & \\
\hline Canada & Finland & 1999 & 2002 & 0.26 & 0.17 & 1.08 & 3.88 & 0.03 & -0.64 & -1.15 \\
\hline Canada & France & 1995 & 1998 & 3.74 & 4.02 & 0.43 & 3.15 & 0.06 & -1.59 & -2.00 \\
\hline Canada & France & 1996 & 1999 & 7.08 & 13.88 & -0.19 & -2.10 & 0.11 & -2.05 & \\
\hline Canada & France & 1997 & 2000 & 8.41 & 9.17 & -0.41 & -2.28 & 0.08 & -0.56 & \\
\hline Canada & France & 1998 & 2001 & 5.06 & 5.34 & 0.30 & 1.59 & 0.12 & 0.96 & 0.05 \\
\hline Canada & France & 1999 & 2002 & 1.72 & 1.26 & 0.84 & 3.13 & 0.04 & -0.86 & \\
\hline Canada & Germany & 1995 & 1998 & -7.08 & -4.09 & 1.40 & 7.92 & 0.11 & -0.74 & \\
\hline Canada & Germany & 1996 & 1999 & 5.60 & 2.41 & 0.05 & 0.19 & 0.10 & -1.46 & \\
\hline Canada & Germany & 1997 & 2000 & -1.74 & -0.57 & 0.88 & 2.66 & 0.08 & -2.00 & \\
\hline Canada & Germany & 1998 & 2001 & 6.40 & 3.06 & 0.02 & 0.08 & 0.12 & 0.89 & -0.26 \\
\hline Canada & Germany & 1999 & 2002 & 8.29 & 2.35 & -0.25 & -0.67 & 0.03 & -0.27 & -1.51 \\
\hline Canada & Ireland & 1995 & 1998 & -3.81 & -0.88 & 1.03 & 2.42 & 0.06 & -1.41 & -1.90 \\
\hline Canada & Ireland & 1996 & 1999 & 9.32 & 6.66 & -0.35 & -2.35 & 0.14 & -1.86 & -1.92 \\
\hline Canada & Ireland & 1997 & 2000 & 9.20 & 6.22 & -0.30 & -1.94 & 0.09 & -1.19 & -1.54 \\
\hline Canada & Ireland & 1998 & 2001 & 4.44 & 3.12 & 0.23 & 1.49 & 0.11 & 0.85 & 0.17 \\
\hline Canada & Ireland & 1999 & 2002 & 1.07 & 0.38 & 0.54 & 1.73 & 0.03 & -0.46 & -1.09 \\
\hline Canada & Italy & 1995 & 1998 & 3.79 & 3.35 & 0.27 & 2.51 & 0.05 & -1.58 & -1.94 \\
\hline Canada & Italy & 1996 & 1999 & 7.00 & 15.84 & -0.11 & -2.24 & 0.12 & -2.13 & \\
\hline Canada & Italy & 1997 & 2000 & 9.21 & 21.22 & -0.39 & -6.77 & 0.14 & -0.95 & -1.49 \\
\hline Canada & Italy & 1998 & 01 & 5.69 & 6.83 & 0.13 & 1.06 & 0.12 & 0.62 & -0.23 \\
\hline
\end{tabular}




\begin{tabular}{|c|c|c|c|c|c|c|c|c|c|c|}
\hline & national interest rate $\mathrm{r}$ & sample & eriod & & coir & gration & regression: & $1=\mathrm{a}+\mathrm{b}$ & & \\
\hline$\underline{\mathrm{r}_{1}}$ & $\mathrm{r}_{2}$ & start & end & $\mathrm{a}$ & t-stat(a) & $\mathrm{b}$ & t-stat(b) & DW & DF & $\mathrm{ADF}$ \\
\hline Canada & Italy & 1999 & 2002 & 0.01 & 0.00 & 0.98 & 2.78 & 0.03 & -0.32 & \\
\hline Canada & Netherlands & 1995 & 1998 & -1.54 & -2.08 & 2.21 & 11.12 & 0.18 & -1.51 & \\
\hline Canada & Netherlands & 1996 & 1999 & 1.48 & 1.22 & 1.33 & 3.78 & 0.14 & -1.74 & \\
\hline Canada & Netherlands & 1997 & 2000 & 2.49 & 4.47 & 1.00 & 7.03 & 0.14 & -1.43 & \\
\hline Canada & Netherlands & 1998 & 2001 & 5.41 & 8.48 & 0.27 & 1.82 & 0.11 & 1.09 & 0.25 \\
\hline Canada & Netherlands & 1999 & 2002 & 3.05 & 3.07 & 0.67 & 2.94 & 0.03 & -0.76 & -1.35 \\
\hline Canada & Portugal & 1995 & 1998 & 3.32 & 4.36 & 0.31 & 4.41 & 0.08 & -0.82 & \\
\hline Canada & Portugal & 1996 & 1999 & 6.89 & 16.86 & -0.10 & -2.18 & 0.12 & -2.15 & \\
\hline Canada & Portugal & 1997 & 2000 & 9.03 & 21.12 & -0.39 & -6.46 & 0.19 & -1.21 & \\
\hline Canada & Portugal & 1998 & 2001 & 5.20 & 6.47 & 0.22 & 1.71 & 0.12 & 0.33 & -0.30 \\
\hline Canada & Portugal & 1999 & 2002 & -1.68 & -1.11 & 1.38 & 5.07 & 0.12 & -1.17 & \\
\hline Canada & Spain & 1995 & 1998 & 3.37 & 4.94 & 0.43 & 4.89 & 0.07 & -1.08 & \\
\hline Canada & Spain & 1996 & 1999 & 6.46 & 15.02 & -0.07 & -1.02 & 0.11 & -1.84 & \\
\hline Canada & Spain & 1997 & 2000 & 8.63 & 11.47 & -0.45 & -3.08 & 0.11 & -0.70 & \\
\hline Canada & Spain & 1998 & 2001 & 4.37 & 5.33 & 0.45 & 2.69 & 0.13 & 0.51 & \\
\hline Canada & Spain & 1999 & 2002 & 2.25 & 1.85 & 0.78 & 3.07 & 0.05 & -0.92 & -1.15 \\
\hline Canada & Australia & 1995 & 1998 & 0.41 & 0.22 & 0.62 & 3.40 & 0.05 & -1.24 & -1.70 \\
\hline Canada & Australia & 1996 & 1999 & 7.32 & 6.38 & -0.14 & -1.12 & 0.11 & -1.79 & \\
\hline Canada & Australia & 1997 & 2000 & 5.62 & 2.54 & 0.08 & 0.33 & 0.06 & -1.42 & \\
\hline Canada & Australia & 1998 & 2001 & -1.70 & -1.11 & 0.92 & 5.40 & 0.16 & 0.49 & \\
\hline Canada & Australia & 1999 & 2002 & -7.67 & -3.50 & 1.52 & 6.22 & 0.05 & -1.09 & \\
\hline Canada & Japan & 1995 & 1998 & 1.65 & 2.41 & 2.78 & 7.36 & 0.10 & -1.05 & \\
\hline Canada & Japan & 1996 & 1999 & 10.48 & 5.71 & -2.84 & -2.42 & 0.13 & -1.89 & \\
\hline Canada & Japan & 1997 & 2000 & 13.10 & 8.21 & -4.48 & -4.25 & 0.12 & -1.20 & -1.55 \\
\hline Canada & Japan & 1998 & 2001 & 3.00 & 1.69 & 2.45 & 2.01 & 0.12 & 0.40 & -0.33 \\
\hline Canada & Japan & 1999 & 2002 & -12.98 & -2.77 & 13.51 & 4.04 & 0.11 & -0.99 & \\
\hline Canada & Switzerland & 1995 & 1998 & -0.73 & -0.46 & 1.54 & 4.68 & 0.07 & -1.00 & \\
\hline Canada & Switzerland & 1996 & 1999 & 8.78 & 7.35 & -0.63 & -2.30 & 0.12 & -2.10 & -2.06 \\
\hline Canada & Switzerland & 1997 & 2000 & 12.18 & 5.82 & -1.40 & -2.79 & 0.09 & -0.42 & -0.96 \\
\hline Canada & Switzerland & 1998 & 2001 & 1.96 & 0.84 & 1.11 & 1.96 & 0.13 & 0.60 & 0.20 \\
\hline Canada & Switzerland & 1999 & 2002 & -5.14 & -1.77 & 2.70 & 3.83 & 0.05 & -1.04 & \\
\hline Canada & United Kingdom & 1995 & 1998 & 1.50 & 0.51 & 0.67 & 1.73 & 0.04 & -2.15 & \\
\hline Canada & United Kingdom & 1996 & 1999 & 5.60 & 4.82 & 0.06 & 0.38 & 0.10 & -1.50 & \\
\hline Canada & United Kingdom & 1997 & 2000 & 7.07 & 5.50 & -0.10 & -0.57 & 0.07 & -1.37 & \\
\hline Canada & United Kingdom & 1998 & 2001 & 3.01 & 4.01 & 0.51 & 4.76 & 0.14 & -0.95 & \\
\hline Canada & United Kingdom & 1999 & 2002 & -3.62 & -10.99 & 1.57 & 29.25 & 0.64 & -3.40 & -3.83 \\
\hline Canada & USA & 1995 & 1998 & -21.56 & -3.95 & 3.32 & 5.16 & 0.11 & -1.22 & \\
\hline Canada & USA & 1996 & 1999 & 13.21 & 3.48 & -0.87 & -1.89 & 0.14 & -1.78 & -1.78 \\
\hline Canada & USA & 1997 & 2000 & 0.46 & 0.22 & 0.69 & 2.89 & 0.07 & -1.60 & \\
\hline Canada & USA & 1998 & 2001 & 0.98 & 2.63 & 0.69 & 15.18 & 0.44 & -3.11 & \\
\hline Canada & USA & 1999 & 2002 & 1.00 & 5.49 & 0.69 & 28.08 & 0.30 & -1.77 & -2.31 \\
\hline Japan & Austria & 1995 & 1998 & -1.07 & -3.04 & 0.40 & 8.13 & 0.11 & -2.75 & -3.26 \\
\hline Japan & Austria & 1996 & 1999 & 0.63 & 7.84 & 0.14 & 11.64 & 0.13 & -1.53 & -1.82 \\
\hline Japan & Austria & 1997 & 2000 & 0.46 & 2.82 & 0.17 & 6.44 & 0.08 & -0.38 & \\
\hline Japan & Austria & 1998 & 2001 & 0.83 & 4.78 & 0.10 & 3.57 & 0.10 & -1.73 & -1.91 \\
\hline Japan & Austria & 1999 & 2002 & 1.03 & 13.58 & 0.06 & 4.90 & 0.36 & -3.15 & -4.16 \\
\hline Japan & Belgium & 1995 & 1998 & -2.49 & -8.02 & 0.57 & 13.80 & 0.51 & -3.58 & -2.29 \\
\hline Japan & Belgium & 1996 & 1999 & -0.48 & -1.84 & 0.29 & 7.78 & 0.24 & -1.12 & \\
\hline Japan & Belgium & 1997 & 2000 & 1.46 & 7.15 & 0.01 & 0.23 & 0.04 & -1.11 & -1.36 \\
\hline Japan & Belgium & 1998 & 2001 & 1.49 & 10.81 & 0.00 & -0.24 & 0.09 & -1.68 & -1.99 \\
\hline Japan & Belgium & 1999 & 2002 & 1.22 & 17.74 & 0.02 & 2.59 & 0.29 & -2.90 & -3.67 \\
\hline Japan & Finland & 1995 & 1998 & 0.09 & 0.35 & 0.28 & 6.96 & 0.11 & -2.99 & -4.57 \\
\hline Japan & Finland & 1996 & 1999 & 0.90 & 9.16 & 0.12 & 6.72 & 0.09 & -1.12 & -0.97 \\
\hline Japan & Finland & 1997 & 2000 & 0.99 & 5.48 & 0.10 & 2.84 & 0.05 & -0.74 & -0.77 \\
\hline Japan & Finland & 1998 & 2001 & 1.22 & 8.92 & 0.04 & 1.69 & 0.09 & -1.77 & -2.03 \\
\hline Japan & Finland & 1999 & 2002 & 1.15 & 21.90 & 0.05 & 4.75 & 0.35 & -3.10 & -4.27 \\
\hline Japan & France & 1995 & 1998 & 1.01 & 4.12 & 0.12 & 3.21 & 0.08 & -4.17 & -3.99 \\
\hline Japan & France & 1996 & 1999 & 1.29 & 26.97 & 0.05 & 5.90 & 0.07 & -0.16 & -0.82 \\
\hline Japan & France & 1997 & 2000 & 1.17 & 11.37 & 0.07 & 3.27 & 0.07 & -0.81 & -1.03 \\
\hline Japan & France & 1998 & 2001 & 1.45 & 12.84 & 0.00 & 0.03 & 0.09 & -1.69 & -1.99 \\
\hline Japan & France & 1999 & 2002 & 1.26 & 23.98 & 0.03 & 2.79 & 0.34 & -3.04 & -3.88 \\
\hline Japan & Germany & 1995 & 1998 & -1.67 & -3.44 & 0.35 & 7.12 & 0.10 & -2.74 & -3.62 \\
\hline Japan & Germany & 1996 & 1999 & 0.52 & 2.28 & 0.11 & 4.55 & 0.07 & -0.12 & -0.68 \\
\hline Japan & Germany & 1997 & 2000 & 1.40 & 3.63 & 0.01 & 0.27 & 0.04 & -1.08 & -1.35 \\
\hline Japan & Germany & 1998 & 2001 & 1.68 & 6.98 & -0.02 & -0.94 & 0.09 & -1.66 & -2.04 \\
\hline Japan & Germany & 1999 & 2002 & 1.15 & 8.91 & 0.03 & 1.98 & 0.28 & -2.84 & -3.59 \\
\hline Japan & Ireland & 1995 & 1998 & -1.85 & -1.71 & 0.36 & 3.35 & 0.10 & -3.94 & \\
\hline Japan & Ireland & 1996 & 1999 & 0.50 & 6.42 & 0.11 & 13.75 & 0.29 & -1.58 & -2.00 \\
\hline Japan & Ireland & 1997 & 2000 & 0.44 & 4.85 & 0.11 & 11.74 & 0.26 & -1.88 & -1.43 \\
\hline Japan & Ireland & 1998 & 2001 & 0.68 & 5.44 & 0.08 & 6.27 & 0.18 & -1.69 & \\
\hline Japan & Ireland & 1999 & 2002 & 1.08 & 10.91 & 0.04 & 3.23 & 0.32 & -3.16 & -3.99 \\
\hline Japan & Italy & 1995 & 1998 & 1.01 & 3.39 & 0.07 & 2.62 & 0.06 & -4.53 & -2.86 \\
\hline Japan & Italy & 1996 & 1999 & 1.26 & 40.26 & 0.03 & 9.76 & 0.10 & -1.05 & -1.07 \\
\hline Japan & Italy & 1997 & 2000 & 1.07 & 36.07 & 0.06 & 15.15 & 0.26 & -2.25 & -2.10 \\
\hline Japan & Italy & 1998 & 2001 & 0.88 & 18.56 & 0.09 & 12.23 & 0.38 & -2.22 & -3.14 \\
\hline Japan & Italy & 1999 & 2002 & 1.04 & 15.01 & 0.06 & 5.21 & 0.37 & -2.87 & -3.89 \\
\hline Japan & Netherlands & 1995 & 1998 & -0.19 & -0.81 & 0.54 & 8.48 & 0.18 & -2.00 & -3.04 \\
\hline Japan & Netherlands & 1996 & 1999 & 1.67 & 10.24 & -0.03 & -0.69 & 0.04 & 0.26 & 0.02 \\
\hline Japan & Netherlands & 1997 & 2000 & 1.66 & 18.04 & -0.04 & -1.69 & 0.06 & -0.93 & -1.37 \\
\hline Japan & Netherlands & 1998 & 2001 & 1.51 & 19.75 & -0.01 & -0.72 & 0.09 & -1.63 & -1.99 \\
\hline Japan & Netherlands & 1999 & 2002 & 1.27 & 35.27 & 0.03 & 3.71 & 0.33 & -3.17 & -4.01 \\
\hline Japan & Portugal & 1995 & 1998 & 0.79 & 4.17 & 0.09 & 5.44 & 0.10 & -2.77 & -3.93 \\
\hline Japan & Portugal & 1996 & 1999 & 1.30 & 41.42 & 0.03 & 8.63 & 0.12 & -1.11 & -1.00 \\
\hline Japan & Portugal & 1997 & 2000 & 1.10 & 32.78 & 0.06 & 12.36 & 0.29 & -2.39 & \\
\hline Japan & Portugal & 1998 & 2001 & 0.89 & 18.65 & 0.09 & 11.77 & 0.53 & -2.72 & \\
\hline Japan & Portugal & 1999 & 2002 & 1.09 & 20.12 & 0.06 & 5.85 & 0.55 & -3.19 & \\
\hline
\end{tabular}


Table A7: Rolling cointegration of short-term corporate lending rates

\begin{tabular}{|c|c|c|c|c|c|c|c|c|c|c|}
\hline & national interest rate $\mathrm{r}$ & sample & eriod & & coin & gration $\mathrm{r}$ & egression: & $=\mathrm{a}+\mathrm{b}$ & & \\
\hline $\mathrm{r}_{1}$ & $\mathrm{r}_{2}$ & start & end & $\mathrm{a}$ & t-stat(a) & $\mathrm{b}$ & t-stat(b) & DW & DF & $\mathrm{ADF}$ \\
\hline Japan & Spain & 1995 & 1998 & 1.03 & 5.38 & 0.10 & 4.06 & 0.07 & -3.69 & -4.41 \\
\hline Japan & Spain & 1996 & 1999 & 1.33 & 35.57 & 0.04 & 6.42 & 0.08 & -0.56 & -0.98 \\
\hline Japan & Spain & 1997 & 2000 & 1.04 & 15.21 & 0.09 & 6.96 & 0.17 & -1.44 & \\
\hline Japan & Spain & 1998 & 2001 & 1.18 & 12.54 & 0.06 & 2.98 & 0.12 & -1.74 & \\
\hline Japan & Spain & 1999 & 2002 & 1.25 & 27.64 & 0.03 & 3.33 & 0.35 & -3.18 & \\
\hline Japan & Australia & 1995 & 1998 & 0.13 & 0.27 & 0.16 & 3.42 & 0.07 & -3.69 & -2.68 \\
\hline Japan & Australia & 1996 & 1999 & 1.06 & 9.15 & 0.05 & 4.34 & 0.06 & 0.10 & -0.31 \\
\hline Japan & Australia & 1997 & 2000 & 1.37 & 5.25 & 0.01 & 0.52 & 0.04 & -1.03 & -1.31 \\
\hline Japan & Australia & 1998 & 2001 & 1.42 & 6.26 & 0.00 & 0.13 & 0.09 & -1.70 & -1.99 \\
\hline Japan & Australia & 1999 & 2002 & 0.98 & 10.44 & 0.05 & 4.45 & 0.34 & -3.43 & \\
\hline Japan & Canada & 1995 & 1998 & 0.49 & 2.76 & 0.19 & 7.36 & 0.11 & -2.52 & \\
\hline Japan & Canada & 1996 & 1999 & 1.80 & 17.97 & -0.04 & -2.42 & 0.06 & -0.34 & -0.89 \\
\hline Japan & Canada & 1997 & 2000 & 1.91 & 20.10 & -0.06 & -4.25 & 0.10 & -1.00 & -1.48 \\
\hline Japan & Canada & 1998 & 2001 & 1.24 & 11.41 & 0.03 & 2.01 & 0.09 & -2.15 & -2.12 \\
\hline Japan & Canada & 1999 & 2002 & 1.29 & 44.16 & 0.02 & 4.04 & 0.34 & -2.67 & -3.48 \\
\hline Japan & Switzerland & 1995 & 1998 & -0.42 & -1.09 & 0.46 & 5.72 & 0.09 & -3.30 & -2.61 \\
\hline Japan & Switzerland & 1996 & 1999 & 0.88 & 7.99 & 0.16 & 6.21 & 0.08 & -0.08 & -0.63 \\
\hline Japan & Switzerland & 1997 & 2000 & 0.59 & 2.56 & 0.22 & 3.96 & 0.08 & -0.84 & -1.13 \\
\hline Japan & Switzerland & 1998 & 2001 & 1.31 & 4.66 & 0.03 & 0.49 & 0.09 & -1.65 & -2.02 \\
\hline Japan & Switzerland & 1999 & 2002 & 0.96 & 8.90 & 0.11 & 4.08 & 0.35 & -3.05 & -3.88 \\
\hline Japan & United Kingdom & 1995 & 1998 & 1.62 & 2.02 & 0.02 & 0.20 & 0.05 & -4.52 & -4.66 \\
\hline Japan & United Kingdom & 1996 & 1999 & 0.97 & 9.12 & 0.08 & 5.62 & 0.08 & -0.50 & -0.25 \\
\hline Japan & United Kingdom & 1997 & 2000 & 0.71 & 7.34 & 0.11 & 8.24 & 0.16 & -2.24 & -2.70 \\
\hline Japan & United Kingdom & 1998 & 2001 & 0.85 & 14.76 & 0.09 & 10.54 & 0.33 & -1.85 & -2.66 \\
\hline Japan & United Kingdom & 1999 & 2002 & 1.21 & 25.49 & 0.03 & 4.00 & 0.34 & -2.48 & -3.49 \\
\hline Japan & USA & 1995 & 1998 & -6.38 & -4.69 & 0.96 & 5.99 & 0.19 & -4.77 & -2.30 \\
\hline Japan & USA & 1996 & 1999 & -0.39 & -1.07 & 0.24 & 5.32 & 0.10 & 1.16 & 0.30 \\
\hline Japan & USA & 1997 & 2000 & 1.60 & 6.08 & -0.01 & -0.33 & 0.05 & -1.10 & -1.39 \\
\hline Japan & USA & 1998 & 2001 & 1.23 & 12.28 & 0.03 & 2.24 & 0.09 & -1.84 & -2.06 \\
\hline Japan & USA & 1999 & 2002 & 1.31 & 50.43 & 0.01 & 3.46 & 0.32 & -2.69 & -3.56 \\
\hline Switzerland & Austria & 1995 & 1998 & -0.17 & -0.73 & 0.69 & 21.43 & 0.25 & -1.95 & \\
\hline Switzerland & Austria & 1996 & 1999 & 0.10 & 0.36 & 0.66 & 15.24 & 0.23 & -1.55 & \\
\hline Switzerland & Austria & 1997 & 2000 & 1.19 & 4.40 & 0.47 & 11.04 & 0.32 & -2.85 & \\
\hline Switzerland & Austria & 1998 & 2001 & 1.65 & 7.79 & 0.40 & 11.82 & 0.41 & -2.28 & \\
\hline Switzerland & Austria & 1999 & 2002 & 1.29 & 7.50 & 0.46 & 16.37 & 0.53 & -1.55 & \\
\hline Switzerland & Belgium & 1995 & 1998 & 0.11 & 0.16 & 0.62 & 6.68 & 0.14 & -0.87 & -1.25 \\
\hline Switzerland & Belgium & 1996 & 1999 & -0.91 & -0.59 & 0.75 & 3.42 & 0.07 & -1.05 & -1.51 \\
\hline Switzerland & Belgium & 1997 & 2000 & 2.67 & 6.43 & 0.21 & 3.66 & 0.10 & -3.43 & \\
\hline Switzerland & Belgium & 1998 & 2001 & 2.28 & 20.64 & 0.24 & 16.90 & 0.73 & -4.26 & \\
\hline Switzerland & Belgium & 1999 & 2002 & 2.18 & 10.33 & 0.25 & 9.19 & 0.25 & -0.02 & 0.60 \\
\hline Switzerland & Finland & 1995 & 1998 & 1.78 & 8.51 & 0.48 & 14.39 & 0.09 & -0.67 & -1.48 \\
\hline Switzerland & Finland & 1996 & 1999 & 0.92 & 2.92 & 0.64 & 10.92 & 0.12 & -1.48 & -2.05 \\
\hline Switzerland & Finland & 1997 & 2000 & 2.13 & 6.33 & 0.39 & 6.08 & 0.12 & -3.20 & -2.38 \\
\hline Switzerland & Finland & 1998 & 2001 & 2.21 & 20.83 & 0.36 & 18.24 & 0.63 & -3.43 & \\
\hline Switzerland & Finland & 1999 & 2002 & 2.14 & 20.94 & 0.38 & 19.36 & 0.59 & -1.63 & -1.94 \\
\hline Switzerland & France & 1995 & 1998 & 2.38 & 19.58 & 0.36 & 19.84 & 0.49 & -3.77 & \\
\hline Switzerland & France & 1996 & 1999 & 2.58 & 50.14 & 0.31 & 35.31 & 1.36 & -4.86 & \\
\hline Switzerland & France & 1997 & 2000 & 2.51 & 28.52 & 0.33 & 19.19 & 1.17 & -4.63 & \\
\hline Switzerland & France & 1998 & 2001 & 2.63 & 27.23 & 0.30 & 15.70 & 1.23 & -4.98 & \\
\hline Switzerland & France & 1999 & 2002 & 2.47 & 20.01 & 0.33 & 13.34 & 0.89 & -2.87 & \\
\hline Switzerland & Germany & 1995 & 1998 & -1.53 & -4.94 & 0.64 & 20.32 & 0.24 & -1.76 & \\
\hline Switzerland & Germany & 1996 & 1999 & -2.95 & -5.91 & 0.79 & 14.65 & 0.21 & -1.87 & \\
\hline Switzerland & Germany & 1997 & 2000 & 0.45 & 0.65 & 0.41 & 5.32 & 0.11 & -2.95 & \\
\hline Switzerland & Germany & 1998 & 2001 & 0.94 & 4.16 & 0.34 & 14.10 & 0.46 & -3.18 & -1.55 \\
\hline Switzerland & Germany & 1999 & 2002 & 1.14 & 2.41 & 0.31 & 6.27 & 0.14 & 0.98 & \\
\hline Switzerland & Ireland & 1995 & 1998 & 2.05 & 1.25 & 0.27 & 1.65 & 0.03 & -1.20 & \\
\hline Switzerland & Ireland & 1996 & 1999 & 2.34 & 3.37 & 0.21 & 2.89 & 0.04 & -1.52 & \\
\hline Switzerland & Ireland & 1997 & 2000 & 2.48 & 7.39 & 0.18 & 5.08 & 0.13 & -2.68 & \\
\hline Switzerland & Ireland & 1998 & 2001 & 2.63 & 9.09 & 0.16 & 5.25 & 0.15 & -1.31 & -2.20 \\
\hline Switzerland & Ireland & 1999 & 2002 & 1.00 & 3.82 & 0.34 & 11.84 & 0.24 & 0.22 & \\
\hline Switzerland & Italy & 1995 & 1998 & 2.02 & 11.64 & 0.26 & 15.96 & 0.13 & -2.67 & -2.25 \\
\hline Switzerland & Italy & 1996 & 1999 & 2.86 & 42.42 & 0.17 & 22.91 & 0.28 & -1.19 & -1.40 \\
\hline Switzerland & Italy & 1997 & 2000 & 3.39 & 28.96 & 0.11 & 6.91 & 0.13 & -1.02 & \\
\hline Switzerland & Italy & 1998 & 2001 & 3.69 & 18.45 & 0.07 & 2.25 & 0.10 & -1.05 & -1.68 \\
\hline Switzerland & Italy & 1999 & 2002 & 1.56 & 8.29 & 0.42 & 13.51 & 0.31 & -0.78 & \\
\hline Switzerland & Netherlands & 1995 & 1998 & 3.35 & 6.99 & 0.38 & 2.95 & 0.03 & 0.22 & -0.54 \\
\hline Switzerland & Netherlands & 1996 & 1999 & 7.32 & 13.15 & -0.87 & -5.35 & 0.17 & -2.36 & \\
\hline Switzerland & Netherlands & 1997 & 2000 & 3.85 & 18.08 & 0.09 & 1.57 & 0.08 & -3.11 & -2.04 \\
\hline Switzerland & Netherlands & 1998 & 2001 & 3.10 & 54.99 & 0.24 & 18.73 & 0.80 & -5.65 & \\
\hline Switzerland & Netherlands & 1999 & 2002 & 2.89 & 36.98 & 0.28 & 15.69 & 0.47 & -1.45 & \\
\hline Switzerland & Portugal & 1995 & 1998 & 2.59 & 36.20 & 0.21 & 30.99 & 0.95 & -3.78 & -3.61 \\
\hline Switzerland & Portugal & 1996 & 1999 & 2.97 & 52.42 & 0.17 & 25.35 & 0.58 & -2.38 & -1.69 \\
\hline Switzerland & Portugal & 1997 & 2000 & 3.37 & 32.13 & 0.12 & 7.89 & 0.16 & -0.86 & \\
\hline Switzerland & Portugal & 1998 & 2001 & 3.68 & 18.80 & 0.08 & 2.37 & 0.12 & -1.17 & -1.74 \\
\hline Switzerland & Portugal & 1999 & 2002 & 2.05 & 12.93 & 0.37 & 13.01 & 0.85 & -3.88 & \\
\hline Switzerland & Spain & 1995 & 1998 & 2.80 & 31.63 & 0.26 & 22.69 & 0.38 & -2.41 & \\
\hline Switzerland & Spain & 1996 & 1999 & 2.97 & 46.76 & 0.23 & 22.79 & 0.62 & -3.44 & \\
\hline Switzerland & Spain & 1997 & 2000 & 2.81 & 29.37 & 0.27 & 14.44 & 0.68 & -3.04 & \\
\hline Switzerland & Spain & 1998 & 2001 & 2.89 & 24.16 & 0.25 & 10.54 & 0.61 & -2.89 & \\
\hline Switzerland & Spain & 1999 & 2002 & 2.66 & 23.61 & 0.30 & 12.93 & 0.75 & -2.48 & -1.29 \\
\hline Switzerland & Australia & 1995 & 1998 & 0.02 & 0.06 & 0.47 & 17.31 & 0.26 & -1.99 & -2.78 \\
\hline Switzerland & Australia & 1996 & 1999 & 0.53 & 2.99 & 0.41 & 21.75 & 0.48 & -2.52 & -2.65 \\
\hline Switzerland & Australia & 1997 & 2000 & 0.89 & 2.49 & 0.36 & 9.19 & 0.25 & -2.69 & \\
\hline Switzerland & Australia & 1998 & 2001 & 1.57 & 5.05 & 0.29 & 8.32 & 0.27 & -2.32 & -1.87 \\
\hline Switzerland & Australia & 1999 & 2002 & 1.51 & 3.94 & 0.29 & 6.82 & 0.20 & -0.04 & -0.90 \\
\hline Switzerland & Canada & 1995 & 1998 & 3.37 & 11.17 & 0.21 & 4.68 & 0.05 & 0.14 & \\
\hline
\end{tabular}


Table A7: Rolling cointegration of short-term corporate lending rates

\begin{tabular}{|c|c|c|c|c|c|c|c|c|c|c|}
\hline & national interest rate $\mathrm{r}$ & sample & geriod & & coin & egration 1 & regression & $=\mathrm{a}+\mathrm{b}$ & & \\
\hline $\mathrm{r}_{1}$ & $\mathrm{r}_{2}$ & start & end & $\mathrm{a}$ & t-stat(a) & $\mathrm{b}$ & t-stat(b) & DW & DF & $\overline{\mathrm{ADF}}$ \\
\hline Switzerland & Canada & 1996 & 1999 & 5.34 & 12.28 & -0.16 & -2.30 & 0.04 & -2.34 & -2.17 \\
\hline Switzerland & Canada & 1997 & 2000 & 4.84 & 20.30 & -0.10 & -2.79 & 0.10 & -1.29 & -1.71 \\
\hline Switzerland & Canada & 1998 & 2001 & 3.68 & 15.72 & 0.07 & 1.96 & 0.11 & -1.35 & -1.21 \\
\hline Switzerland & Canada & 1999 & 2002 & 3.57 & 25.17 & 0.09 & 3.83 & 0.11 & -0.38 & -1.17 \\
\hline Switzerland & Japan & 1995 & 1998 & 3.15 & 11.06 & 0.90 & 5.72 & 0.06 & -0.62 & -1.40 \\
\hline Switzerland & Japan & 1996 & 1999 & -0.20 & -0.27 & 2.92 & 6.21 & 0.07 & -1.39 & \\
\hline Switzerland & Japan & 1997 & 2000 & 2.43 & 5.48 & 1.16 & 3.96 & 0.11 & -1.87 & -1.38 \\
\hline Switzerland & Japan & 1998 & 2001 & 3.91 & 8.47 & 0.16 & 0.49 & 0.10 & -1.14 & -1.40 \\
\hline Switzerland & Japan & 1999 & 2002 & 0.63 & 0.74 & 2.48 & 4.08 & 0.18 & -1.03 & \\
\hline Switzerland & United Kingdom & 1995 & 1998 & 8.28 & 8.38 & -0.46 & -3.59 & 0.05 & -0.25 & \\
\hline Switzerland & United Kingdom & 1996 & 1999 & 4.52 & 7.61 & -0.02 & -0.29 & 0.03 & -1.47 & -1.76 \\
\hline Switzerland & United Kingdom & 1997 & 2000 & 3.68 & 10.70 & 0.07 & 1.48 & 0.08 & -2.39 & -1.65 \\
\hline Switzerland & United Kingdom & 1998 & 2001 & 4.07 & 17.74 & 0.01 & 0.29 & 0.10 & -1.16 & -1.40 \\
\hline Switzerland & United Kingdom & 1999 & 2002 & 3.12 & 14.08 & 0.16 & 4.46 & 0.11 & -0.75 & -0.72 \\
\hline Switzerland & USA & 1995 & 1998 & -4.17 & -1.94 & 1.05 & 4.14 & 0.09 & -1.67 & -1.07 \\
\hline Switzerland & USA & 1996 & 1999 & 0.92 & 0.47 & 0.42 & 1.76 & 0.04 & -1.10 & -1.93 \\
\hline Switzerland & USA & 1997 & 2000 & 2.19 & 4.14 & 0.23 & 3.78 & 0.10 & -3.54 & \\
\hline Switzerland & USA & 1998 & 2001 & 3.79 & 17.10 & 0.04 & 1.57 & 0.10 & -1.19 & -1.34 \\
\hline Switzerland & USA & 1999 & 2002 & 3.65 & 29.74 & 0.06 & 3.78 & 0.10 & -0.53 & -1.42 \\
\hline United Kingdom & Austria & 1995 & 1998 & 8.85 & 11.84 & -0.17 & -1.67 & 0.12 & -0.97 & -2.28 \\
\hline United Kingdom & Austria & 1996 & 1999 & 4.60 & 3.89 & 0.41 & 2.26 & 0.06 & -0.83 & -2.06 \\
\hline United Kingdom & Austria & 1997 & 2000 & 1.02 & 0.79 & 0.99 & 4.86 & 0.08 & -0.94 & -1.96 \\
\hline United Kingdom & Austria & 1998 & 2001 & 1.26 & 0.74 & 0.90 & 3.32 & 0.04 & -0.17 & -0.59 \\
\hline United Kingdom & Austria & 1999 & 2002 & 0.17 & 0.13 & 0.97 & 4.71 & 0.05 & -1.17 & \\
\hline United Kingdom & Belgium & 1995 & 1998 & 6.69 & 6.87 & 0.12 & 0.94 & 0.11 & -1.25 & -1.42 \\
\hline United Kingdom & Belgium & 1996 & 1999 & -7.79 & -3.53 & 2.13 & 6.84 & 0.23 & -2.09 & \\
\hline United Kingdom & Belgium & 1997 & 2000 & 5.34 & 3.77 & 0.27 & 1.37 & 0.06 & -0.60 & -1.84 \\
\hline United Kingdom & Belgium & 1998 & 2001 & 7.92 & 6.02 & -0.13 & -0.78 & 0.05 & -0.11 & -1.94 \\
\hline United Kingdom & Belgium & 1999 & 2002 & 5.19 & 4.31 & 0.12 & 0.76 & 0.04 & -0.57 & \\
\hline United Kingdom & Finland & 1995 & 1998 & 8.05 & 16.44 & -0.07 & -0.90 & 0.11 & -1.04 & -2.27 \\
\hline United Kingdom & Finland & 1996 & 1999 & 5.55 & 5.26 & 0.32 & 1.64 & 0.06 & -0.78 & -2.21 \\
\hline United Kingdom & Finland & 1997 & 2000 & 3.18 & 2.54 & 0.78 & 3.28 & 0.06 & -0.34 & -1.81 \\
\hline United Kingdom & Finland & 1998 & 2001 & 5.28 & 3.94 & 0.30 & 1.22 & 0.04 & -0.10 & -1.14 \\
\hline United Kingdom & Finland & 1999 & 2002 & 2.39 & 2.66 & 0.71 & 4.14 & 0.04 & -1.08 & \\
\hline United Kingdom & France & 1995 & 1998 & 9.05 & 29.20 & -0.22 & -4.77 & 0.18 & -0.94 & -1.49 \\
\hline United Kingdom & France & 1996 & 1999 & 7.48 & 15.15 & -0.04 & -0.44 & 0.06 & -0.51 & -2.25 \\
\hline United Kingdom & France & 1997 & 2000 & 6.29 & 7.90 & 0.19 & 1.26 & 0.06 & -0.90 & -2.46 \\
\hline United Kingdom & France & 1998 & 2001 & 6.78 & 6.23 & 0.02 & 0.11 & 0.05 & -0.06 & -1.63 \\
\hline United Kingdom & France & 1999 & 2002 & 3.21 & 3.89 & 0.58 & 3.53 & 0.07 & -1.37 & \\
\hline United Kingdom & Germany & 1995 & 1998 & 9.52 & 10.08 & -0.20 & -2.03 & 0.12 & -0.97 & -2.20 \\
\hline United Kingdom & Germany & 1996 & 1999 & 7.92 & 3.68 & -0.07 & -0.30 & 0.06 & -0.53 & -2.31 \\
\hline United Kingdom & Germany & 1997 & 2000 & 6.23 & 2.28 & 0.11 & 0.39 & 0.06 & -0.77 & -2.30 \\
\hline United Kingdom & Germany & 1998 & 2001 & 11.04 & 4.89 & -0.44 & -1.84 & 0.06 & -0.19 & -2.12 \\
\hline United Kingdom & Germany & 1999 & 2002 & 7.48 & 3.41 & -0.14 & -0.63 & 0.04 & -0.44 & -1.18 \\
\hline United Kingdom & Ireland & 1995 & 1998 & 1.27 & 0.90 & 0.63 & 4.50 & 0.13 & -1.24 & \\
\hline United Kingdom & Ireland & 1996 & 1999 & -0.35 & -0.45 & 0.80 & 9.79 & 0.16 & -1.49 & \\
\hline United Kingdom & Ireland & 1997 & 2000 & 0.04 & 0.06 & 0.77 & 10.18 & 0.17 & -1.35 & -2.09 \\
\hline United Kingdom & Ireland & 1998 & 2001 & 0.34 & 0.26 & 0.71 & 5.02 & 0.06 & 0.43 & \\
\hline United Kingdom & Ireland & 1999 & 2002 & 2.91 & 1.67 & 0.35 & 1.83 & 0.04 & -0.83 & \\
\hline United Kingdom & Italy & 1995 & 1998 & 9.32 & 25.45 & -0.16 & -4.75 & 0.17 & -0.68 & -2.01 \\
\hline United Kingdom & Italy & 1996 & 1999 & 6.74 & 15.94 & 0.06 & 1.29 & 0.06 & -0.69 & -2.36 \\
\hline United Kingdom & Italy & 1997 & 2000 & 5.19 & 12.84 & 0.28 & 5.28 & 0.08 & -2.00 & -3.14 \\
\hline United Kingdom & Italy & 1998 & 2001 & 2.02 & 3.38 & 0.74 & 8.25 & 0.08 & 0.42 & -0.56 \\
\hline United Kingdom & Italy & 1999 & 2002 & 2.19 & 1.66 & 0.65 & 2.99 & 0.04 & -0.55 & \\
\hline United Kingdom & Netherlands & 1995 & 1998 & 5.87 & 12.82 & 0.47 & 3.84 & 0.13 & -2.02 & -1.53 \\
\hline United Kingdom & Netherlands & 1996 & 1999 & 2.24 & 2.14 & 1.47 & 4.82 & 0.12 & -0.42 & -1.09 \\
\hline United Kingdom & Netherlands & 1997 & 2000 & 7.18 & 10.72 & 0.02 & 0.14 & 0.06 & -0.79 & -2.34 \\
\hline United Kingdom & Netherlands & 1998 & 2001 & 7.52 & 10.26 & -0.15 & -0.86 & 0.06 & -0.03 & -1.55 \\
\hline United Kingdom & Netherlands & 1999 & 2002 & 4.20 & 6.88 & 0.44 & 3.16 & 0.04 & -1.22 & -0.75 \\
\hline United Kingdom & Portugal & 1995 & 1998 & 8.64 & 28.97 & -0.10 & -3.57 & 0.15 & -0.88 & -2.24 \\
\hline United Kingdom & Portugal & 1996 & 1999 & 6.93 & 17.56 & 0.04 & 0.91 & 0.06 & -0.64 & -2.42 \\
\hline United Kingdom & Portugal & 1997 & 2000 & 5.49 & 13.28 & 0.26 & 4.45 & 0.09 & -1.91 & -2.88 \\
\hline United Kingdom & Portugal & 1998 & 2001 & 1.96 & 3.49 & 0.81 & 8.90 & 0.20 & -0.97 & -1.36 \\
\hline United Kingdom & Portugal & 1999 & 2002 & 1.08 & 1.20 & 0.91 & 5.57 & 0.17 & -1.50 & \\
\hline United Kingdom & Spain & 1995 & 1998 & 8.49 & 30.23 & -0.12 & -3.26 & 0.13 & -0.89 & -2.31 \\
\hline United Kingdom & Spain & 1996 & 1999 & 7.12 & 17.70 & 0.02 & 0.38 & 0.06 & -0.56 & -2.29 \\
\hline United Kingdom & Spain & 1997 & 2000 & 4.95 & 8.28 & 0.46 & 3.96 & 0.09 & -1.63 & -2.42 \\
\hline United Kingdom & Spain & 1998 & 2001 & 4.19 & 4.65 & 0.55 & 3.03 & 0.06 & -0.23 & -0.91 \\
\hline United Kingdom & Spain & 1999 & 2002 & 3.77 & 5.01 & 0.49 & 3.13 & 0.07 & -1.28 & \\
\hline United Kingdom & Australia & 1995 & 1998 & 10.80 & 18.23 & -0.32 & -5.42 & 0.19 & -0.82 & -2.00 \\
\hline United Kingdom & Australia & 1996 & 1999 & 8.47 & 7.98 & -0.13 & -1.13 & 0.06 & -0.52 & -2.16 \\
\hline United Kingdom & Australia & 1997 & 2000 & 7.93 & 4.29 & -0.07 & -0.36 & 0.06 & -0.83 & -2.36 \\
\hline United Kingdom & Australia & 1998 & 2001 & 4.85 & 2.23 & 0.23 & 0.95 & 0.04 & -0.22 & -1.54 \\
\hline United Kingdom & Australia & 1999 & 2002 & -2.06 & -1.47 & 0.91 & 5.83 & 0.07 & -1.58 & \\
\hline United Kingdom & Canada & 1995 & 1998 & 7.00 & 19.60 & 0.09 & 1.73 & 0.11 & -1.31 & -2.08 \\
\hline United Kingdom & Canada & 1996 & 1999 & 6.96 & 8.36 & 0.05 & 0.38 & 0.06 & -0.51 & -2.18 \\
\hline United Kingdom & Canada & 1997 & 2000 & 7.72 & 9.80 & -0.07 & -0.57 & 0.06 & -0.89 & -2.54 \\
\hline United Kingdom & Canada & 1998 & 2001 & 2.69 & 3.02 & 0.64 & 4.76 & 0.07 & -2.40 & \\
\hline United Kingdom & Canada & 1999 & 2002 & 2.50 & 19.92 & 0.61 & 29.25 & 0.65 & -3.48 & -3.81 \\
\hline United Kingdom & Japan & 1995 & 1998 & 7.54 & 20.09 & 0.04 & 0.20 & 0.10 & -1.15 & -2.21 \\
\hline United Kingdom & Japan & 1996 & 1999 & -0.52 & -0.38 & 5.00 & 5.62 & 0.10 & -1.16 & -1.58 \\
\hline United Kingdom & Japan & 1997 & 2000 & -0.95 & -0.95 & 5.45 & 8.24 & 0.17 & -2.05 & -2.68 \\
\hline United Kingdom & Japan & 1998 & 2001 & -4.87 & -4.35 & 8.10 & 10.54 & 0.29 & -1.07 & -2.04 \\
\hline United Kingdom & Japan & 1999 & 2002 & -5.58 & -1.91 & 8.34 & 4.00 & 0.11 & -0.82 & \\
\hline United Kingdom & Switzerland & 1995 & 1998 & 9.84 & 15.70 & -0.47 & -3.59 & 0.14 & -0.90 & -1.81 \\
\hline United Kingdom & Switzerland & 996 & 999 & 7.60 & 6.51 & -0.08 & -0.29 & 0.06 & -0.52 & -2.29 \\
\hline
\end{tabular}




\begin{tabular}{|c|c|c|c|c|c|c|c|c|c|c|}
\hline & national interest rate $\mathrm{r}$ & sample & eriod & & coin & egration $r$ & egression: & $=\mathrm{a}+\mathrm{b}$ & & \\
\hline $\mathrm{r}_{1}$ & $\mathrm{r}_{2}$ & start & end & $\mathrm{a}$ & t-stat(a) & $\mathrm{b}$ & t-stat(b) & DW & $\mathrm{DF}$ & $\mathrm{ADF}$ \\
\hline United Kingdom & Switzerland & 1997 & 2000 & 4.55 & 2.46 & 0.65 & 1.48 & 0.06 & -0.99 & -2.26 \\
\hline United Kingdom & Switzerland & 1998 & 2001 & 6.12 & 2.25 & 0.19 & 0.29 & 0.05 & -0.03 & -1.67 \\
\hline United Kingdom & Switzerland & 1999 & 2002 & -1.61 & -0.93 & 1.88 & 4.46 & 0.07 & -1.61 & -1.27 \\
\hline United Kingdom & USA & 1995 & 1998 & 1.00 & 0.43 & 0.78 & 2.82 & 0.08 & -1.07 & -1.82 \\
\hline United Kingdom & USA & 1996 & 1999 & -8.32 & -2.93 & 1.89 & 5.50 & 0.11 & -0.42 & -0.50 \\
\hline United Kingdom & USA & 1997 & 2000 & 5.46 & 2.98 & 0.21 & 0.99 & 0.05 & -0.69 & -2.11 \\
\hline United Kingdom & USA & 1998 & 2001 & 2.84 & 3.47 & 0.50 & 5.02 & 0.05 & -1.56 & -2.10 \\
\hline United Kingdom & USA & 1999 & 2002 & 3.04 & 25.64 & 0.42 & 26.65 & 0.46 & -4.05 & -4.02 \\
\hline USA & Austria & 1995 & 1998 & 6.77 & 24.04 & 0.24 & 6.10 & 0.31 & -1.61 & -1.70 \\
\hline USA & Austria & 1996 & 1999 & 7.00 & 18.79 & 0.19 & 3.40 & 0.16 & -0.96 & -1.86 \\
\hline USA & Austria & 1997 & 2000 & 4.20 & 4.84 & 0.68 & 4.97 & 0.07 & -1.16 & -1.44 \\
\hline USA & Austria & 1998 & 2001 & 2.67 & 1.27 & 0.87 & 2.60 & 0.04 & 2.60 & -0.65 \\
\hline USA & Austria & 1999 & 2002 & -4.38 & -1.43 & 1.90 & 3.80 & 0.02 & 0.24 & -0.88 \\
\hline USA & Belgium & 1995 & 1998 & 6.08 & 18.43 & 0.32 & 7.28 & 0.50 & -1.91 & -0.14 \\
\hline USA & Belgium & 1996 & 1999 & 5.02 & 5.40 & 0.46 & 3.50 & 0.19 & -1.29 & -1.86 \\
\hline USA & Belgium & 1997 & 2000 & 2.85 & 5.60 & 0.78 & 11.17 & 0.27 & -1.79 & -2.18 \\
\hline USA & Belgium & 1998 & 2001 & 7.96 & 5.06 & 0.02 & 0.10 & 0.05 & 2.59 & -0.43 \\
\hline USA & Belgium & 1999 & 2002 & 6.73 & 2.44 & 0.06 & 0.17 & 0.02 & 0.99 & -0.44 \\
\hline USA & Finland & 1995 & 1998 & 7.42 & 40.21 & 0.17 & 5.81 & 0.32 & -1.26 & -1.50 \\
\hline USA & Finland & 1996 & 1999 & 7.64 & 21.82 & 0.12 & 1.80 & 0.15 & -1.08 & -1.84 \\
\hline USA & Finland & 1997 & 2000 & 3.92 & 6.00 & 0.87 & 7.03 & 0.12 & -1.39 & -1.87 \\
\hline USA & Finland & 1998 & 2001 & 5.96 & 3.76 & 0.40 & 1.37 & 0.04 & 2.69 & -0.08 \\
\hline USA & Finland & 1999 & 2002 & 0.07 & 0.03 & 1.36 & 3.33 & 0.01 & 0.47 & -0.39 \\
\hline USA & France & 1995 & 1998 & 7.98 & 46.67 & 0.07 & 2.96 & 0.22 & -0.86 & -2.04 \\
\hline USA & France & 1996 & 1999 & 8.00 & 49.93 & 0.05 & 1.73 & 0.15 & -1.19 & -1.73 \\
\hline USA & France & 1997 & 2000 & 6.42 & 14.20 & 0.41 & 4.67 & 0.13 & -1.65 & \\
\hline USA & France & 1998 & 2001 & 6.41 & 5.05 & 0.34 & 1.37 & 0.05 & 2.65 & 0.39 \\
\hline USA & France & 1999 & 2002 & 1.85 & 0.94 & 1.07 & 2.75 & 0.03 & 0.06 & \\
\hline USA & Germany & 1995 & 1998 & 6.56 & 16.66 & 0.20 & 4.88 & 0.28 & -1.25 & -1.54 \\
\hline USA & Germany & 1996 & 1999 & 7.33 & 10.39 & 0.10 & 1.32 & 0.14 & -1.14 & -1.78 \\
\hline USA & Germany & 1997 & 2000 & -2.44 & -2.70 & 1.20 & 12.12 & 0.19 & -1.64 & -2.78 \\
\hline USA & Germany & 1998 & 2001 & 9.27 & 3.34 & -0.12 & -0.41 & 0.05 & 2.40 & -0.59 \\
\hline USA & Germany & 1999 & 2002 & 12.68 & 2.56 & -0.57 & -1.11 & 0.02 & 0.76 & -0.72 \\
\hline USA & Ireland & 1995 & 1998 & 3.54 & 8.74 & 0.49 & 12.17 & 0.75 & -3.28 & \\
\hline USA & Ireland & 1996 & 1999 & 5.78 & 21.21 & 0.26 & 9.17 & 0.24 & -0.30 & -1.70 \\
\hline USA & Ireland & 1997 & 2000 & 6.19 & 7.72 & 0.25 & 2.90 & 0.04 & 0.40 & -0.42 \\
\hline USA & Ireland & 1998 & 2001 & 5.40 & 2.85 & 0.29 & 1.44 & 0.04 & 2.93 & -1.02 \\
\hline USA & Ireland & 1999 & 2002 & 1.99 & 0.49 & 0.58 & 1.29 & 0.01 & 0.94 & -0.97 \\
\hline USA & Italy & 1995 & 1998 & 7.90 & 39.02 & 0.05 & 2.87 & 0.21 & -0.94 & -2.18 \\
\hline USA & Italy & 1996 & 1999 & 7.88 & 60.01 & 0.04 & 3.03 & 0.16 & -0.97 & -1.78 \\
\hline USA & Italy & 1997 & 2000 & 8.46 & 24.36 & 0.01 & 0.14 & 0.05 & 0.41 & -0.72 \\
\hline USA & Italy & 1998 & 2001 & 6.82 & 6.19 & 0.20 & 1.19 & 0.05 & 2.54 & -0.32 \\
\hline USA & Italy & 1999 & 2002 & 0.55 & 0.18 & 1.10 & 2.14 & 0.02 & 0.82 & -0.31 \\
\hline USA & Netherlands & 1995 & 1998 & 7.21 & 40.62 & 0.34 & 7.21 & 0.40 & -1.17 & \\
\hline USA & Netherlands & 1996 & 1999 & 7.89 & 18.54 & 0.11 & 0.88 & 0.14 & -1.33 & -1.65 \\
\hline USA & Netherlands & 1997 & 2000 & 5.99 & 23.62 & 0.66 & 10.09 & 0.17 & -1.60 & \\
\hline USA & Netherlands & 1998 & 2001 & 7.18 & 8.27 & 0.22 & 1.11 & 0.04 & 2.76 & 0.05 \\
\hline USA & Netherlands & 1999 & 2002 & 3.69 & 2.56 & 0.82 & 2.47 & 0.02 & 0.34 & -0.65 \\
\hline USA & Portugal & 1995 & 1998 & 7.91 & 55.65 & 0.05 & 4.08 & 0.26 & -1.35 & -1.91 \\
\hline USA & Portugal & 1996 & 1999 & 7.95 & 64.32 & 0.04 & 2.68 & 0.16 & -1.01 & -1.76 \\
\hline USA & Portugal & 1997 & 2000 & 8.43 & 25.20 & 0.01 & 0.23 & 0.05 & 0.40 & -0.72 \\
\hline USA & Portugal & 1998 & 2001 & 6.16 & 5.81 & 0.32 & 1.87 & 0.05 & 2.08 & -1.07 \\
\hline USA & Portugal & 1999 & 2002 & -2.56 & -1.14 & 1.77 & 4.37 & 0.08 & -0.80 & -1.11 \\
\hline USA & Spain & 1995 & 1998 & 7.99 & 59.34 & 0.06 & 3.69 & 0.25 & -1.06 & -1.78 \\
\hline USA & Spain & 1996 & 1999 & 8.00 & 62.32 & 0.04 & 2.12 & 0.15 & -1.10 & -1.78 \\
\hline USA & Spain & 1997 & 2000 & 7.17 & 16.92 & 0.26 & 3.21 & 0.06 & -0.71 & -0.77 \\
\hline USA & Spain & 1998 & 2001 & 5.71 & 5.11 & 0.49 & 2.18 & 0.05 & 2.17 & 0.03 \\
\hline USA & Spain & 1999 & 2002 & 2.73 & 1.54 & 0.95 & 2.56 & 0.03 & 0.04 & -0.39 \\
\hline USA & Australia & 1995 & 1998 & 7.69 & 21.69 & 0.08 & 2.24 & 0.20 & -0.75 & -1.87 \\
\hline USA & Australia & 1996 & 1999 & 7.85 & 22.17 & 0.04 & 1.19 & 0.14 & -1.17 & -1.73 \\
\hline USA & Australia & 1997 & 2000 & 1.80 & 2.34 & 0.74 & 8.74 & 0.15 & -2.37 & -2.53 \\
\hline USA & Australia & 1998 & 2001 & -3.28 & -1.65 & 1.27 & 5.75 & 0.06 & 2.14 & 1.60 \\
\hline USA & Australia & 1999 & 2002 & -11.15 & -3.45 & 2.05 & 5.69 & 0.04 & 0.31 & -0.01 \\
\hline USA & Canada & 1995 & 1998 & 7.75 & 53.60 & 0.11 & 5.16 & 0.25 & -0.30 & -0.15 \\
\hline USA & Canada & 1996 & 1999 & 8.77 & 32.71 & -0.08 & -1.89 & 0.18 & -1.58 & -1.89 \\
\hline USA & Canada & 1997 & 2000 & 7.10 & 14.40 & 0.22 & 2.89 & 0.05 & 0.01 & -1.50 \\
\hline USA & Canada & 1998 & 2001 & 0.17 & 0.32 & 1.21 & 15.18 & 0.37 & -2.95 & \\
\hline USA & Canada & 1999 & 2002 & -0.98 & -3.27 & 1.38 & 28.08 & 0.29 & -1.48 & -1.68 \\
\hline USA & Japan & 1995 & 1998 & 7.66 & 55.36 & 0.46 & 5.99 & 0.33 & -1.98 & -1.13 \\
\hline USA & Japan & 1996 & 1999 & 5.75 & 12.13 & 1.61 & 5.32 & 0.21 & -0.59 & -1.27 \\
\hline USA & Japan & 1997 & 2000 & 8.86 & 8.31 & -0.23 & -0.33 & 0.05 & 0.40 & -0.76 \\
\hline USA & Japan & 1998 & 2001 & 2.91 & 1.24 & 3.59 & 2.24 & 0.05 & 2.14 & -1.26 \\
\hline USA & Japan & 1999 & 2002 & -16.65 & -2.41 & 17.04 & 3.46 & 0.07 & -0.43 & -0.92 \\
\hline USA & Switzerland & 1995 & 1998 & 7.25 & 24.34 & 0.26 & 4.14 & 0.25 & -1.10 & -1.79 \\
\hline USA & Switzerland & 1996 & 1999 & 7.60 & 20.13 & 0.15 & 1.76 & 0.15 & -1.16 & -1.82 \\
\hline USA & Switzerland & 1997 & 2000 & 4.27 & 3.82 & 1.01 & 3.78 & 0.07 & -1.28 & -1.75 \\
\hline USA & Switzerland & 1998 & 2001 & 3.17 & 1.00 & 1.20 & 1.57 & 0.04 & 2.73 & 0.05 \\
\hline USA & Switzerland & 1999 & 2002 & -8.35 & -2.02 & 3.79 & 3.78 & 0.03 & -0.58 & -1.36 \\
\hline USA & United Kingdom & 1995 & 1998 & 7.04 & 13.77 & 0.19 & 2.82 & 0.16 & -0.16 & -1.22 \\
\hline USA & United Kingdom & 1996 & 1999 & 6.74 & 24.09 & 0.21 & 5.50 & 0.19 & -1.15 & -1.20 \\
\hline USA & United Kingdom & 1997 & 2000 & 7.79 & 10.78 & 0.10 & 0.99 & 0.04 & 0.62 & -0.48 \\
\hline USA & United Kingdom & 1998 & 2001 & 3.25 & 3.31 & 0.71 & 5.02 & 0.05 & 0.70 & -0.41 \\
\hline USA & United Kingdom & 1999 & 2002 & -6.28 & -12.30 & 2.21 & 26.65 & 0.43 & -3.72 & -3.51 \\
\hline Panel B: Cointeg & tion relative to rest of eurozone & & & & & & & & & \\
\hline Austria & eurozone average excluding Aus & 1995 & 1998 & 2.20 & 5.84 & 0.72 & 13.21 & 0.08 & -2.74 & -3.51 \\
\hline Austria & eurozone average excluding Austria & 1996 & 1999 & 2.78 & 16.50 & 0.61 & 22.29 & 0.23 & -1.66 & \\
\hline
\end{tabular}


Table A7: Rolling cointegration of short-term corporate lending rates

\begin{tabular}{|c|c|c|c|c|c|c|c|c|c|c|c|c|c|c|}
\hline \multicolumn{3}{|c|}{ national interest rate $r$} & \multicolumn{2}{|c|}{ sample period } & & coin & gration & regression: & $=\mathrm{a}+\mathrm{b}$ & & & cointe- & $\mathrm{EC}$ & \\
\hline$r_{1}$ & $\mathrm{r}_{2}$ & & start & end & $\mathrm{a}$ & t-stat(a) & $\mathrm{b}$ & t-stat(b) & DW & $\mathrm{DF}$ & $\mathrm{ADF}$ & gration? & speed & t-stat \\
\hline Austria & eurozone average excluding & Austria & 1997 & 2000 & 1.82 & 5.42 & 0.79 & 13.32 & 0.17 & -0.93 & & no & -0.12 & -1.58 \\
\hline Austria & eurozone average excluding & Austria & 1998 & 2001 & -0.66 & -2.23 & 1.26 & 23.41 & 0.54 & -2.73 & & yes & -0.30 & -2.40 \\
\hline Austria & eurozone average excluding & Austria & 1999 & 2002 & -1.44 & -4.15 & 1.40 & 21.69 & 0.44 & -2.44 & -1.86 & yes & -0.25 & -2.55 \\
\hline Belgium & eurozone average excluding & Belgium & 1995 & 1998 & 4.89 & 10.23 & 0.31 & 5.52 & 0.21 & -2.10 & -5.20 & yes & -0.08 & -1.48 \\
\hline Belgium & eurozone average excluding & Belgium & 1996 & 1999 & 6.18 & 31.06 & 0.12 & 4.53 & 0.27 & -1.94 & -2.15 & no & -0.17 & -2.22 \\
\hline Belgium & eurozone average excluding & Belgium & 1997 & 2000 & 4.71 & 6.00 & 0.37 & 3.27 & 0.08 & -0.01 & -0.65 & no & -0.09 & -1.88 \\
\hline Belgium & eurozone average excluding & Belgium & 1998 & 2001 & -0.83 & -1.19 & 1.24 & 12.15 & 0.19 & -2.47 & -2.64 & no & -0.24 & -2.76 \\
\hline Belgium & eurozone average excluding & Belgium & 1999 & 2002 & -1.34 & -3.67 & 1.36 & 24.89 & 0.73 & -3.25 & -2.75 & yes & -0.48 & -3.52 \\
\hline Finland & eurozone average excluding & Finland & 1995 & 1998 & -0.13 & -0.29 & 0.74 & 13.96 & 0.05 & -1.19 & -1.56 & no & -0.09 & -2.35 \\
\hline Finland & eurozone average excluding & Finland & 1996 & 1999 & 2.04 & 9.48 & 0.44 & 15.64 & 0.11 & -2.32 & -2.01 & no & -0.09 & -1.98 \\
\hline Finland & eurozone average excluding & Finland & 1997 & 2000 & 2.02 & 4.32 & 0.46 & 6.93 & 0.07 & -0.13 & -1.77 & no & -0.07 & -1.69 \\
\hline Finland & eurozone average excluding & Finland & 1998 & 2001 & -0.95 & -3.23 & 0.92 & 21.57 & 0.26 & -2.39 & & no & -0.25 & -2.47 \\
\hline Finland & eurozone average excluding & Finland & 1999 & 2002 & -1.70 & -5.03 & 1.03 & 20.58 & 0.17 & -1.38 & & no & -0.08 & -1.03 \\
\hline France & eurozone average excluding & France & 1995 & 1998 & -3.68 & -7.60 & 1.15 & 21.42 & 0.53 & -3.26 & & yes & -0.21 & -2.30 \\
\hline France & eurozone average excluding & France & 1996 & 1999 & -3.37 & -8.44 & 1.13 & 22.76 & 0.36 & -2.19 & -2.32 & no & -0.16 & -1.86 \\
\hline France & eurozone average excluding & France & 1997 & 2000 & -1.67 & -2.58 & 0.91 & 10.49 & 0.29 & -1.96 & -2.11 & no & -0.26 & -3.05 \\
\hline France & eurozone average excluding & France & 1998 & 2001 & -1.77 & -2.57 & 0.93 & 9.87 & 0.34 & -2.38 & & no & -0.21 & -2.26 \\
\hline France & eurozone average excluding & France & 1999 & 2002 & -2.87 & -5.47 & 1.09 & 15.02 & 0.66 & -3.02 & & yes & -0.44 & -3.50 \\
\hline Germany & eurozone average excluding & Germany & 1995 & 1998 & 5.76 & 22.34 & 0.51 & 15.86 & 0.17 & -2.80 & & no & -0.17 & -1.93 \\
\hline Germany & eurozone average excluding & Germany & 1996 & 1999 & 7.20 & 53.91 & 0.30 & 15.63 & 0.28 & -2.14 & & no & -0.21 & -2.38 \\
\hline Germany & eurozone average excluding & Germany & 1997 & 2000 & 8.03 & 22.66 & 0.19 & 3.19 & 0.04 & 2.96 & 0.80 & no & -0.02 & -0.51 \\
\hline Germany & eurozone average excluding & Germany & 1998 & 2001 & 5.88 & 10.88 & 0.61 & 6.47 & 0.04 & -1.63 & & no & -0.05 & -1.61 \\
\hline Germany & eurozone average excluding & Germany & 1999 & 2002 & 5.52 & 14.26 & 0.73 & 10.41 & 0.09 & -0.96 & & no & 0.00 & -0.04 \\
\hline Ireland & eurozone average excluding & Ireland & 1995 & 1998 & 9.37 & 19.81 & 0.08 & 1.45 & 0.22 & -0.63 & -1.69 & no & -0.14 & -1.79 \\
\hline Ireland & eurozone average excluding & Ireland & 1996 & 1999 & 7.04 & 10.74 & 0.33 & 3.81 & 0.08 & -0.87 & -1.52 & no & -0.06 & -1.50 \\
\hline Ireland & eurozone average excluding & Ireland & 1997 & 2000 & 2.17 & 2.97 & 1.05 & 9.97 & 0.17 & -2.18 & -2.45 & no & -0.09 & -1.36 \\
\hline Ireland & eurozone average excluding & Ireland & 1998 & 2001 & 1.13 & 1.57 & 1.20 & 11.37 & 0.20 & -1.80 & -2.25 & no & -0.18 & -2.51 \\
\hline Ireland & eurozone average excluding & Ireland & 1999 & 2002 & 1.84 & 10.58 & 1.07 & 41.46 & 0.97 & -4.22 & & yes & -0.54 & -3.72 \\
\hline Italy & eurozone average excluding & Italy & 1995 & 1998 & -2.66 & -3.44 & 1.66 & 17.19 & 0.06 & -1.43 & -0.93 & no & 0.02 & 0.28 \\
\hline Italy & eurozone average excluding & Italy & 1996 & 1999 & -10.73 & -14.69 & 2.74 & 26.96 & 0.17 & -0.98 & & no & -0.01 & -0.13 \\
\hline Italy & eurozone average excluding & Italy & 1997 & 2000 & -5.68 & -2.08 & 1.91 & 4.78 & 0.02 & 0.14 & -0.69 & no & 0.01 & 0.37 \\
\hline Italy & eurozone average excluding & Italy & 1998 & 2001 & 2.46 & 1.55 & 0.60 & 2.59 & 0.02 & -3.80 & -1.90 & yes & -0.06 & -2.37 \\
\hline Italy & eurozone average excluding & Italy & 1999 & 2002 & 0.40 & 1.18 & 0.82 & 16.79 & 0.25 & -4.17 & & yes & -0.12 & -1.18 \\
\hline Netherlands & eurozone average excluding & Netherlands & 1995 & 1998 & 2.47 & 4.55 & 0.14 & 2.23 & 0.05 & -2.14 & -1.80 & no & -0.06 & -1.22 \\
\hline Netherlands & eurozone average excluding & Netherlands & 1996 & 1999 & 4.43 & 18.52 & -0.13 & -4.26 & 0.26 & -2.09 & & no & -0.14 & -2.01 \\
\hline Netherlands & eurozone average excluding & Netherlands & 1997 & 2000 & 3.03 & 3.03 & 0.11 & 0.81 & 0.06 & 0.24 & -0.70 & no & -0.09 & -1.79 \\
\hline Netherlands & eurozone average excluding & Netherlands & 1998 & 2001 & -3.87 & -4.41 & 1.16 & 9.27 & 0.15 & -2.44 & -2.50 & no & -0.22 & -3.12 \\
\hline Netherlands & eurozone average excluding & Netherlands & 1999 & 2002 & -5.29 & -15.55 & 1.40 & 28.27 & 1.13 & -3.95 & -1.79 & yes & -0.56 & -3.68 \\
\hline Portugal & eurozone average excluding & Portugal & 1995 & 1998 & -7.12 & -11.73 & 2.09 & 29.32 & 0.76 & -4.58 & -4.16 & yes & -0.54 & -3.97 \\
\hline Portugal & eurozone average excluding & Portugal & 1996 & 1999 & -7.83 & -18.47 & 2.17 & 38.56 & 0.96 & -3.72 & & yes & -0.40 & -3.12 \\
\hline Portugal & eurozone average excluding & Portugal & 1997 & 2000 & -8.29 & -6.22 & 2.19 & 11.43 & 0.19 & -0.77 & & no & -0.06 & -0.86 \\
\hline Portugal & eurozone average excluding & Portugal & 1998 & 2001 & -0.73 & -0.56 & 1.00 & 5.28 & 0.11 & -2.52 & -2.04 & no & -0.16 & -2.97 \\
\hline Portugal & eurozone average excluding & Portugal & 1999 & 2002 & -0.17 & -0.29 & 0.84 & 9.87 & 0.49 & -2.90 & -2.30 & yes & -0.29 & -2.11 \\
\hline Spain & eurozone average excluding & Spain & 1995 & 1998 & -7.31 & -19.62 & 1.72 & 40.09 & 0.77 & -3.30 & & yes & -0.33 & -3.01 \\
\hline Spain & eurozone average excluding & Spain & 1996 & 1999 & -6.09 & -17.51 & 1.58 & 35.04 & 0.76 & -4.01 & -2.73 & yes & -0.33 & -2.43 \\
\hline Spain & eurozone average excluding & Spain & 1997 & 2000 & -4.10 & -11.25 & 1.29 & 25.38 & 1.23 & -4.80 & -5.77 & yes & -0.76 & -4.84 \\
\hline Spain & eurozone average excluding & Spain & 1998 & 2001 & -3.35 & -7.46 & 1.17 & 18.44 & 1.10 & -4.10 & & yes & -0.56 & -3.93 \\
\hline Spain & eurozone average excluding & Spain & 1999 & 2002 & -3.65 & -8.44 & 1.20 & 19.42 & 1.26 & -4.59 & -2.87 & yes & -0.67 & -5.80 \\
\hline Australia & eurozone average & & 1995 & 1998 & 2.97 & 9.74 & 0.84 & 23.48 & 0.29 & -1.90 & & no & -0.15 & -2.03 \\
\hline Australia & eurozone average & & 1996 & 1999 & 3.30 & 10.82 & 0.82 & 20.21 & 0.23 & -1.51 & & no & -0.12 & -1.64 \\
\hline Australia & eurozone average & & 1997 & 2000 & 5.04 & 7.95 & 0.58 & 6.40 & 0.11 & -0.64 & -1.26 & no & -0.18 & -2.68 \\
\hline Australia & eurozone average & & 1998 & 2001 & 4.69 & 5.95 & 0.63 & 5.46 & 0.11 & -1.25 & -1.68 & no & -0.16 & -2.44 \\
\hline Australia & eurozone average & & 1999 & 2002 & 4.01 & 5.37 & 0.74 & 6.67 & 0.13 & -1.26 & -1.62 & no & -0.14 & -2.39 \\
\hline Canada & eurozone average & & 1995 & 1998 & 0.95 & 0.76 & 0.67 & 4.55 & 0.06 & -1.06 & -1.59 & no & -0.04 & -0.88 \\
\hline Canada & eurozone average & & 1996 & 1999 & 7.30 & 9.38 & -0.17 & -1.63 & 0.11 & -1.98 & & no & -0.08 & -1.41 \\
\hline Canada & eurozone average & & 1997 & 2000 & 10.34 & 7.89 & -0.58 & -3.06 & 0.09 & -0.49 & -1.02 & no & -0.04 & -0.76 \\
\hline Canada & eurozone average & & 1998 & 2001 & 4.39 & 3.01 & 0.32 & 1.49 & 0.11 & 0.90 & 0.08 & no & 0.08 & 1.38 \\
\hline Canada & eurozone average & & 1999 & 2002 & 1.55 & 0.69 & 0.65 & 1.95 & 0.03 & -0.43 & & no & -0.06 & -2.04 \\
\hline Japan & eurozone average & & 1995 & 1998 & 0.32 & 0.95 & 0.17 & 4.40 & 0.07 & -3.81 & -2.87 & yes & -0.13 & -2.88 \\
\hline Japan & eurozone average & & 1996 & 1999 & 1.08 & 17.39 & 0.06 & 7.78 & 0.07 & -0.62 & -0.70 & no & -0.02 & -0.42 \\
\hline Japan & eurozone average & & 1997 & 2000 & 0.66 & 5.74 & 0.12 & 7.51 & 0.10 & -0.82 & -1.27 & no & -0.03 & -0.56 \\
\hline Japan & eurozone average & & 1998 & 2001 & 1.03 & 6.36 & 0.06 & 2.65 & 0.10 & -1.58 & -1.85 & no & -0.11 & -2.14 \\
\hline Japan & eurozone average & & 1999 & 2002 & 1.13 & 14.23 & 0.04 & 3.40 & 0.32 & -3.07 & -3.96 & yes & -0.20 & -2.12 \\
\hline Switzerland & eurozone average & & 1995 & 1998 & 1.11 & 9.33 & 0.43 & 31.00 & 0.36 & -2.57 & & no & -0.23 & -2.24 \\
\hline Switzerland & eurozone average & & 1996 & 1999 & 1.68 & 18.39 & 0.36 & 29.57 & 0.46 & -2.34 & & yes & -0.23 & -2.12 \\
\hline Switzerland & eurozone average & & 1997 & 2000 & 1.74 & 11.46 & 0.35 & 16.16 & 0.42 & -2.68 & & yes & -0.30 & -2.93 \\
\hline Switzerland & eurozone average & & 1998 & 2001 & 1.88 & 11.14 & 0.33 & 13.42 & 0.44 & -2.36 & & yes & -0.44 & -4.30 \\
\hline Switzerland & eurozone average & & 1999 & 2002 & 1.55 & 7.75 & 0.38 & 12.86 & 0.34 & -0.20 & 1.26 & no & 0.18 & 1.45 \\
\hline United Kingdom & eurozone average & & 1995 & 1998 & 9.35 & 18.88 & -0.21 & -3.55 & 0.14 & -0.84 & -2.17 & no & -0.05 & -1.07 \\
\hline United Kingdom & eurozone average & & 1996 & 1999 & 6.77 & 9.18 & 0.07 & 0.69 & 0.06 & -0.60 & -2.31 & no & -0.07 & -2.13 \\
\hline United Kingdom & eurozone average & & 1997 & 2000 & 3.18 & 3.06 & 0.59 & 3.96 & 0.07 & -1.29 & -2.58 & no & -0.05 & -1.30 \\
\hline United Kingdom & eurozone average & & 1998 & 2001 & 3.71 & 2.31 & 0.47 & 2.00 & 0.04 & 0.21 & -0.74 & no & -0.03 & -0.90 \\
\hline United Kingdom & eurozone average & & 1999 & 2002 & 3.15 & 2.26 & 0.44 & 2.12 & 0.04 & -0.81 & & no & -0.04 & -1.41 \\
\hline USA & eurozone average & & 1995 & 1998 & 7.55 & 31.75 & 0.11 & 3.94 & 0.24 & -1.08 & -1.93 & no & -0.07 & -1.02 \\
\hline USA & eurozone average & & 1996 & 1999 & 7.70 & 33.11 & 0.08 & 2.47 & 0.15 & -1.06 & -1.79 & no & -0.10 & -1.80 \\
\hline USA & eurozone average & & 1997 & 2000 & 5.99 & 8.26 & 0.36 & 3.49 & 0.05 & -0.73 & -1.04 & no & -0.20 & -3.46 \\
\hline USA & eurozone average & & 1998 & 2001 & 5.87 & 3.00 & 0.33 & 1.15 & 0.04 & 2.84 & 0.00 & no & -0.01 & -0.17 \\
\hline USA & eurozone average & & 1999 & 2002 & 2.42 & 0.74 & 0.71 & 1.48 & 0.01 & 0.92 & -0.16 & no & -0.01 & -0.70 \\
\hline
\end{tabular}

